\author{
University of Brasilia \\ Department of Mathematics \\ PhD Program
}

\title{
An Abstract Linking Theorem Applied to Indefinite Problems via Spectral Properties
}

by

Mayra Soares Costa Rodrigues 
University of Brasilia

Department of Mathematics

PhD Program

\section{An Abstract Linking Theorem Applied to Indefinite Problems via Spectral Properties}

by

\section{Mayra Soares Costa Rodrigues ${ }^{\text {}}$}

Thesis presented to Department of Mathematics of Brasilia, as part of the requisites to obtain the degree of

\section{PHD IN MATHEMATICS}

Brasilia, December $07^{\text {th }} 2018$

Examination Committee:

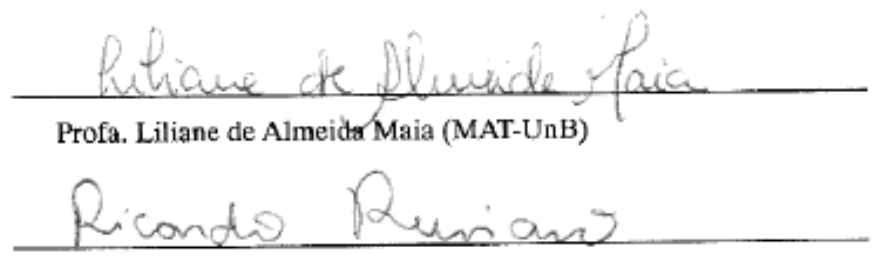

Prof. Dr. Ricardo Ruviaro (MAT-UnB)

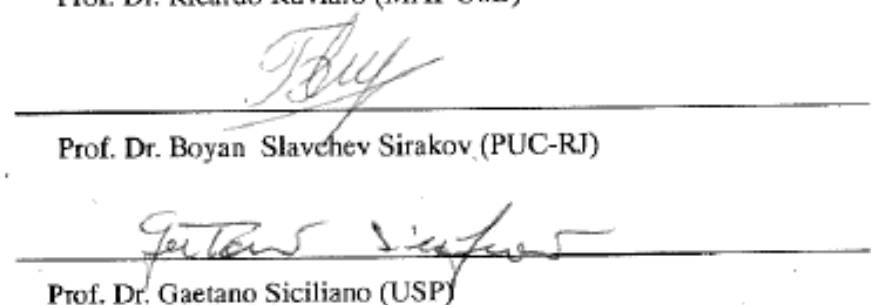

${ }^{*}$ The author has had financial support from PICME (CAPES) and CNPq during the elaboration of this work. 
Ficha catalográfica elaborada automaticamente, com os dados fornecidos pelo(a) autor(a)

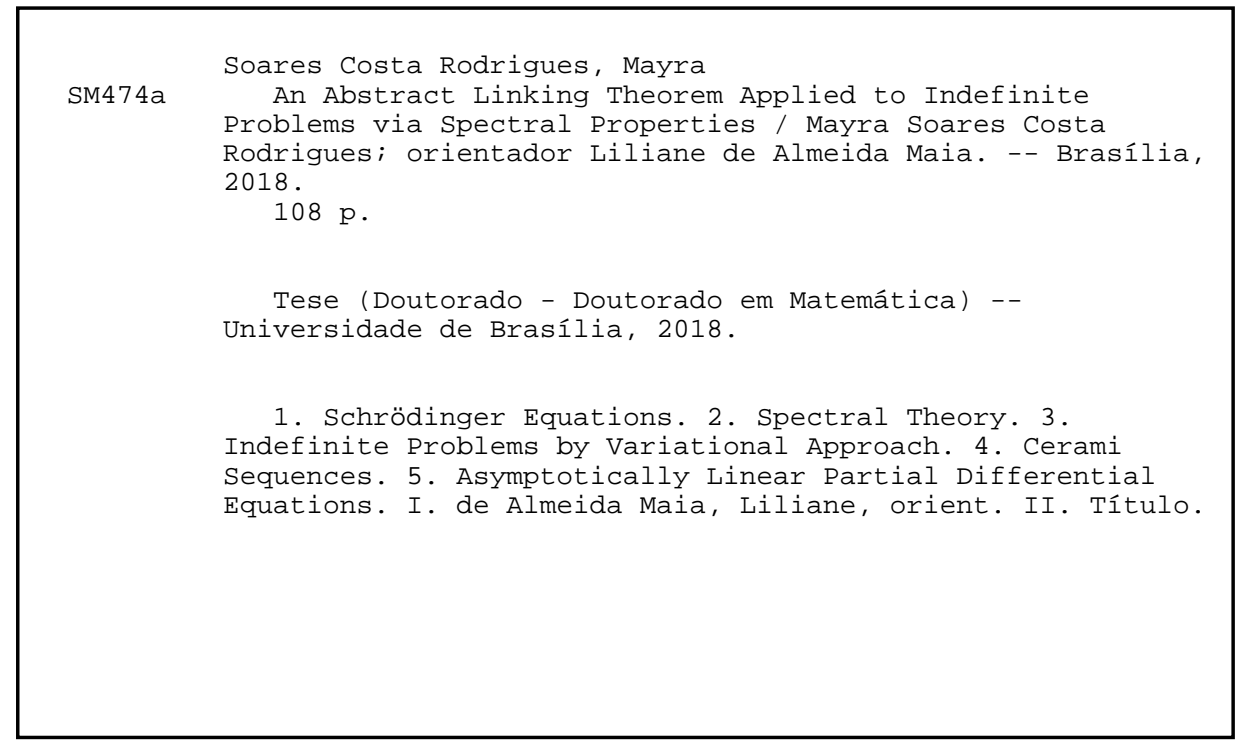


"For of Him, and through Him, and to Him, are all things, to whom be glory for ever. Amen!" Roman 11:36 


\section{Acknowledgments}

First of all I give thanks to my Holy God, who always surprises me, teaching me that I need to trust in His mercy and goodness, even when everything seems to be against my dreams and plans. I would never be here without Him guiding me.

I thank my family, mainly my patient and lovely husband Marcos Vinícius S. Rodrigues, who have been by my side during this whole trajectory. I also highlight my mother Rosangela S. da Silva and my maternal grandparents João S. da Silva and Maria de L. da Silva, who supported me since the beginning of my academic career. They always were a great motivation to go on in hard times.

Thanks to my advisor, for too many things she has taught me, for supporting me and encouraging me to give my best and never give up. I appreciate all her effort.

I extend my thanks to the other professors in the Department of Mathematics of the University of Brasilia, who participated of my academic training. I also thanks all the staff of this department, without them nothing done here would be possible.

I still want to thank $C A P E S$ and $C N P q$ for the financial support throughout my doctorate.

Thanks to my friends who cheered for me and believed that I could reach my goals. Especially those who prayed for me and stayed with me when I needed them most.

Finally, I thank to everyone who has been present in my life during this period and has contributed in some way, so that my work could be accomplished. I worship my Lord and salvator of my life, Jesus Christ for letting me get here, and for all who has been part of this story. 


\section{Dedication}

To my grandparents João and Maria, even though they were no longer here, they were my inspiration to go on until the end. 


\section{Abstract}

An abstract linking theorem for Cerami sequences is developed with the purpose of handling indefinite problems by variational approach, especially nonlinear Schrödinger equations with sign-changing potentials and asymptotically linear nonlinearities and Hamiltonian Systems. In order to find a nontrivial solution to the problem under discussion, an indefinite functional is associated to this problem and a nontrivial critical point to such a functional is obtained applying the abstract result. This critical point is going to be a weak solution to the problem, as desired.

Spectral theory is the main tool used in this work. Exploiting spectral properties of the elliptic operator associated to the problem in question, it enables to establish a linking structure on the Hilbert space where the mentioned critical points are sought.

Key words: Spectral Theory, Linking Geometry, Cerami Sequences, Asymptotically Linear, Indefinite Operator, Nonlinear Schrödinger Equation, Hamiltonian Systems, Non-cooperative Elliptic Systems. 


\section{Resumo}

Um teorema abstrato de linking para sequências de Cerami é desenvolvido com o propósito de lidar com problemas indefinidos por meio da abordagem variacional, em particular equações de Schrödinger com potencial que muda de sinal e não linearidades assintoticamente lineares e sistemas Hamiltonianos. Com o objetivo de encontrar uma solução não trivial para o problema sob discussão, um funcional indefinido é associado a este problema e um ponto crítico não trivial para tal funcional é obtido aplicando o resultado abstrato. Este ponto crítico será uma solução fraca para o problema, como desejado.

A teoria espectral é principal ferramenta usada nesse trabalho. Explorando propriedades espectrais do operador elíptico associado ao problema em questão, é possível estabelecer uma estrutura de linking no espaço de Hilbert onde os pontos críticos mencionados são procurados.

Palavras-chave: Teoria Espectral, Geometria de Linking, Sequências de Cerami, Assintoticamente Linear, Operadores Indefinidos, Equação de Schrödinger, Sistemas Hamiltonianos, Sistemas Elípticos Não-cooperativos. 


\section{Notation}

$B_{R}$

$B_{R}(x)$

$B_{R}[x]$

$u_{n} \rightarrow u$

$u_{n} \rightarrow u$

$u_{n} \rightarrow u$, a. e. in $\Omega$

$\nabla u=\left(\frac{\partial u}{\partial x_{1}}, \ldots, \frac{\partial u}{\partial x_{N}}\right)$

$\frac{\partial u}{\partial \eta}=\eta \cdot \nabla u$

$\Delta u=\sum_{i=1}^{N} \frac{\partial^{2} u}{\partial x_{i}^{2}}$

$\omega \subset \subset \Omega$

$|\Omega|$

$\bar{\Omega}$

$\partial \Omega$

$\operatorname{diam}(\Omega)$ closed ball of radius $R$ centered in zero;

open ball of radius $R$ centered in $x$;

closed ball of radius $R$ centered in $x$;

strong convergence (in norm);

weak convergence;

convergence almost everywhere in $\Omega$;

gradient of $u$;

exterior normal derivative;

Laplacian of $\mathrm{u}$;

$\bar{\omega}$ is compact and it is a subset of $\Omega$;

measure of $\Omega$;

closure of $\Omega$;

boundary of $\Omega$;

diameter of $\Omega$; 
$p^{\prime}=\frac{p}{p-1}$

$f=o(g), \quad$ when $x \rightarrow x_{0}$

$\operatorname{supp} f$

$C(X, Y)$

$C^{1}(X, Y)$

$X^{\prime}$

$L^{p}(\Omega)$

$L_{\mathrm{loc}}^{p}(\Omega)$

$L_{\mathrm{h}(\mathrm{x})}^{p}(\Omega)$

$W^{k, p}(\Omega)$

$H^{1}(\Omega)$

$H^{-1}(\Omega)$

$H^{2}(\Omega)$

$H_{\mathrm{loc}}^{2}(\Omega)$

$\|u\|_{H^{1}(\Omega)}=\left(\|\nabla u\|_{L^{2}(\Omega)}^{2}+\|u\|_{L^{2}(\Omega)}^{2}\right)^{1 / 2}$

$\|u\|_{L^{p}(\Omega)}=\left(\int_{\Omega}|u|^{p} d x\right)^{1 / p}, 1 \leq p<+\infty$

$\|u\|_{L_{h(x)}^{p}(\Omega)}=\left(\int_{\Omega} h(x)|u|^{p} d x\right)^{1 / p}, 1 \leq p<+\infty$ usual norm of $L_{h(x)}^{p}(\Omega), 1 \leq p<+\infty ;$

$\|u\|_{\infty}=\sup _{x \in \mathbb{R}^{N}} \operatorname{ess}|u(x)|$

$|\cdot|$ conjugate exponent of $p$;

$\lim _{x \rightarrow x_{0}} \frac{|f(x)|}{g(x)}=0$

support of $f$;

continuous functions from $X$ to $Y$;

continuously differentiable

functions from $X$ to $Y$;

dual space of $X$;

Lebesgue functions $p$-integrable;

$L_{\mathrm{loc}}^{p}(\Omega)=\left\{u \in L^{p}\left(\Omega^{\prime}\right), \quad \forall \Omega^{\prime} \subset \subset \Omega\right\}$

$L_{\mathrm{h}(\mathrm{x})}^{p}(\Omega)=\left\{u: \Omega \rightarrow \mathbb{R}: h(x)|u|^{p} \in L^{1}(\Omega)\right\}$

$W^{k, p}(\Omega)=\left\{u \in L^{p}(\Omega): D^{\alpha} u \in L^{p}(\Omega), \quad \forall|\alpha| \leq k\right\}$

Sobolev space $W^{1,2}(\Omega)$;

dual space of $H^{1}(\Omega)$;

Sobolev space $W^{2,2}(\Omega)$;

$W_{\text {loc }}^{2,2}(\Omega)=\left\{u \in W^{2,2}\left(\Omega^{\prime}\right), \quad \forall \Omega^{\prime} \subset \subset \Omega\right\} ;$

usual norm of $H^{1}\left(\mathbb{R}^{N}\right)$;

usual norm of $L^{p}(\Omega)$;

usual norm of $L^{\infty}\left(\mathbb{R}^{N}\right)$;

$\mathbb{R}^{N}$ norm. 


\section{Contents}

Introduction 1

1 An Abstract Linking Theorem for Cerami Sequences 12

1.1 Notion of Linking and Some Definitions . . . . . . . . . . . . . . . . . . . 12

1.2 A Quantitative Deformation Lemma . . . . . . . . . . . . . . . . . . . . 15

1.3 Proof of the Main Result . . . . . . . . . . . . . . . . . . . . . . . . . . 24

2 Applications on Indefinite Problems 26

2.1 An Asymptotically Quadratic Hamiltonian System via an Abstract Linking

Theorem . . . . . . . . . . . . . . . . . . . . . . 26

2.1 .1 Variational Structure . . . . . . . . . . . . . . . . . . . . 27

2.1 .2 Setting Compactness $\ldots \ldots \ldots \ldots$. . . . . . . . . . . . . . . . . 29

2.1 .3 Linking Geometry . . . . . . . . . . . . . . . . . . . . . 30

$2.1 .4 \quad$ Boundedness of Cerami Sequences $\ldots \ldots \ldots 33$

2.2 A Nonlinear Schrödinger Equation Corresponding to a Hamiltonian System . 35

$2.2 .1 \quad$ Variational Structure . . . . . . . . . . . . . . . . . . . . . . . . . . . . 36

$2.2 .2 \quad$ Setting Compactness $\ldots \ldots \ldots \ldots$. . . . . . . . . . . . . . . . . 38

2.2 .3 Linking Geometry . . . . . . . . . . . . . . . . . . . . . . . . . . . . . . . 39

$2.2 .4 \quad$ Boundedness of Cerami Sequences $\ldots \ldots \ldots$. . . . . . . . . . . . 42

2.3 Asymptotically Linear Schrödinger Equations in $\mathbb{R}^{N}$ via an Abstract Linking

Theorem . . . . . . . . . . . . . . . . . . . . . . . . 44

2.3 .1 Variational Setting . . . . . . . . . . . . . . . . . . 46

2.3 .2 Boundedness of Cerami Sequences . . . . . . . . . . . . . . 47

$2.3 .3 \quad$ Setting Some Necessary Hypotheses _ . . . . . . . . . . . . . . . . 52

2.3 .4 Linking Geometry . . . . . . . . . . . . . . . . . . . . 55

2.3.5 A Schrödinger Operator with Purely Absolutely Continuous Spectrum . . 59 
\begin{tabular}{|lll}
3 & A Radial Nonlinear Schrödinger Equation & 64
\end{tabular}

3.1 Variational Setting . . . . . . . . . . . . . . . . . . . . . . . 67

$3.2 \quad$ Weak Continuity and Uniform Differentiation of $\mathrm{I} \ldots \ldots \ldots \ldots$

3.3 Linking Geometry $\ldots \ldots \ldots \ldots \ldots \ldots$

3.4 Boundedness of Cerami Sequences $\ldots \ldots \ldots \ldots \ldots$. . . . . . . . . . 76

$\begin{array}{lll}4 & \text { Non-cooperative Hamiltonian Elliptic Systems in } \mathbb{R}^{N} & \mathbf{7 9}\end{array}$

4.1 Asymptotically Quadratic Elliptic Systems . . . . . . . . . . . . . . . . . . . . 81

4.1 .1 Variational Framework . . . . . . . . . . . . . . . . . . . . . . . . . 82

4.1 .2 Establishing Compactness $\ldots \ldots \ldots \ldots \ldots$. . . . . . . . . . . 84

4.1 .3 Linking Geometry . . . . . . . . . . . . . . . . . . . 86

4.1 .4 Boundedness of Cerami Sequences . . . . . . . . . . . . . . . 88

$4.2 \quad$ Super Quadratic Elliptic Systems $\ldots \ldots \ldots$. . . . . . . . . . . . . . . . . . . . . . . . 91

4.2 .1 Linking Structure . . . . . . . . . . . . . . . . . . . . . . . . . 93

4.2 .2 Boundedness of Cerami Sequences . . . . . . . . . . . . . . . . . . 95

\begin{tabular}{ll}
\hline Auxiliary Results & 101
\end{tabular}

\begin{tabular}{ll}
\hline Bibliography Rerefences & 104
\end{tabular} 


\section{Introduction}

In this work the groundbreaking paper [7] by V. Benci and P.H. Rabinowitz is revisited. The aim is to prove an abstract linking theorem for Cerami sequences [11], which will complement related works found in the literature and make possible to extend for many applications. Our interest in applications are twofold, on one hand, extending results for existence of solutions to nonlinear Schrödinger Equations, Elliptic Systems or even Hamiltonian Systems, with very general potentials which make the problems indefinite. On the other hand working with nonlinear terms which do not satisfy any monotonicity condition such as those required to perform projections on the so called Nehari Manifold as in [40, 54], for instance. In this purpose spectral properties of self-adjoint operators are going to be exploited, in order to get the geometry of a linking structure and then apply an abstract result to obtain a critical point to the functional associated to the nonlinear equation, namely a solution to the problem. Furthermore, a compactness structure given by Cerami sequences, $(C)_{c}$ sequence for short, is faced here, since asymptotically linear problems at infinity are studied. Thus, inspired by the theory developed in [7], in this work a more general version of their main result, Theorem 1.29, is provided for $(C)_{c}$ sequences. To do so, a Deformation Lemma adapted for Cerami sequences is proved and then the abstract results obtained by V. Benci and P. Rabinowitz are extended.

Our main result, developed throughout Chapter 1, is the following:

Linking Theorem for Cerami Sequences: Let $E$ be a real Hilbert space, with inner product $(\cdot, \cdot), E_{1}$ a closed subspace of $E$ and $E_{2}=E_{1}^{\perp}$. Let $I \in C^{1}(E, \mathbb{R})$ satisfying:

$\left(I_{1}\right) \quad I(u)=\frac{1}{2}(L u, u)+B(u)$, for all $u \in E$, where $u=u_{1}+u_{2} \in E_{1} \oplus E_{2}, L u=L_{1} u_{1}+L_{2} u_{2}$ and $L_{i}: E_{i} \rightarrow E_{i}, i=1,2$ is a bounded linear self adjoint mapping.

$\left(I_{2}\right) \quad B$ is weakly continuous and uniformly differentiable on bounded subsets of $E$.

$\left(I_{3}\right)$ There exist Hilbert manifolds $S, Q \subset E$, such that $Q$ is bounded and has boundary $\partial Q$, constants $\alpha>\omega$ and $v \in E_{2}$ such that

(i) $S \subset v+E_{1}$ and $I \geq \alpha$ on $S$; (ii) $I \leq \omega$ on $\partial Q$; (iii) $S$ and $\partial Q$ link.

$\left(I_{4}\right)$ If for a sequence $\left(u_{n}\right), I\left(u_{n}\right)$ is bounded and $\left(1+\left\|u_{n}\right\|\right)\left\|I^{\prime}\left(u_{n}\right)\right\| \rightarrow 0$, as $n \rightarrow+\infty$, then 
$\left(u_{n}\right)$ is bounded.

Then I possesses a critical value $c \geq \alpha$.

It is important to highlight $\left(I_{2}\right)$ implies that $B^{\prime}(u)$ maps weakly convergent to strongly convergent sequences, which gives a kind of partial compactness for $I$. Moreover, $\left(I_{4}\right)$ is a weakened version of Cerami condition, so denoted $(C)_{c}$ condition, once the boundedness of any $(C)_{c}$ sequence will be enough to look for a nontrivial critical point, without wondering whether it has a convergent subsequence. Together, hypotheses $\left(I_{2}\right)$ and $\left(I_{4}\right)$ ensure the existence of a critical value $c$ which can be characterized as a minimax level. Furthermore, hypotheses $\left(I_{1}\right)$ and $\left(I_{3}\right)$ produce a quite general linking geometry for functional $I$, so that both subspaces in the Hilbert's decomposition are allowed to be infinite dimensional. The conjunction of these hypotheses reproduces the sufficient structure to obtain a nontrivial critical point for $I$ in the desired applications scenario.

The pioneering work in this direction is 3 by P. Bartolo, V. Benci and D. Fortunato, where a Deformation Lemma for Cerami sequences was developed assuming $(C)_{c}$ condition, as a qualitative deformation lemma, with the purpose of extending previous critical point results to non super-quadratic problems. Thereafter, D. Costa and C. Magalhães in [15] proved abstract linking results for strongly indefinite non-quadratic problems on bounded domains, making use of the Deformation Lemma introduced in [3] and proving that under their assumptions the associated functional satisfied $(C)_{c}$ condition. Alternatively, as aforementioned, the same lines as [7] are followed, hence a deformation lemma without $(C)_{c}$ condition is proved, and furthermore, our version of linking theorem only requires the boundedness of Cerami sequences.

In the literature one also finds a paper by G. Li and C. Wang [34, which presented a similar argument, introducing a new kind of deformation lemma, without $(C)_{c}$ condition, but subsequently used in a linking theorem under $(C)_{c}$ condition. Moreover, in the abstract result they required that one of the subspaces in the linking decomposition being finite dimension, while in our result both subspaces in the decomposition may be of infinite dimension. Their construction was inspired by ideas of M. Willem [57], for the quantitative deformation lemma. Although a kind of deformation lemma is developed, it is deeply different from theirs, since nonstandard ideas in [7] are closely followed. In fact, the mapping $\eta$ in deformation is in general determined by solving an appropriate initial value problem involving $I^{\prime}(\eta)$. However, this is not suitable for our purposes, because is necessary to construct an $\eta$ satisfying special properties, which will be fundamental in attaining the critical minimax level.

It is also important to mention the theory developed by W. Kryszewski and A. Szulkin in [31], where they solved a more general class of superlinear problems, with assumptions of periodicity. Developing a new degree theory and a weaker topology, they generalized abstract linking theorems introduced in [7] also working with Palais-Smale sequences. Following the same idea, in [32] G. Li and A. Szulkin extended the results in [31] obtaining a $(C)_{c}$ sequence 
for the asymptotically linear case. Nevertheless, so as to get a non-trivial solution, without $(C)_{c}$ condition, these authors required extra assumptions, including a monotonicity condition on the nonlinearity in an auxiliary problem solved in [55], which had been treated by adapting techniques in [31] and [27].

Posteriorly, T. Bartsch and Y. Ding [5] complemented the results in [31] considering both, Palais-Smale and Cerami sequences, in order to apply their abstract results to a Dirac equation where the nonlinearity could be asymptotically linear or superlinear at infinity. Similarly, in [21], Y. Ding and B. Ruf worked with an asymptotically linear problem with a Dirac operator, but without periodicity conditions. Their operator satisfies that the essential spectrum is $\mathbb{R} \backslash(-a, a)$ and that the discrete spectrum intersects the interval $\left(0, q_{0}\right)$, for some positive $q_{0}$. Then they could make use of discrete and positive eigenvalues in the linking structure and apply a particular case of the result in [5], so as to obtain a Cerami sequence. Under their assumptions they were able to prove that their functional satisfied $(C)_{c}$ condition, which yielded their results.

It's worth to highlight that [21] adapted assumptions and arguments introduced in another very inspiring paper [20], where L. Jeanjean and Y. Ding worked with Hamiltonian Systems, looking for homoclinic orbits, without any periodicity condition. These authors also applied the abstract critical point theory developed in [5], and in order to recover the desired compactness they imposed hypotheses controlling the size of the nonlinearity with respect to the behavior of the potential at infinity. Thus, their assumptions yielded the linking geometry, and provided $(C)_{c}$ condition. In contrast to the argument presented by these authors, our approach does not require the guarantee of $(C)_{c}$ condition, the necessary compactness to solve the problem is embedded inside the four conditions assumed in the abstract result.

Still referring to abstract results involving linking structure, it is as well known that M. Schechter and W. Zou have developed many relevant papers in this spirit, see especially [45, 46, 47, 48] among other works by these authors. In our understanding, their results are away from ours in the sense that, roughly speaking, they usually work with weaker linking geometries in order to get either a Palais-Smale or a Cerami bounded sequence. Then they apply widely alternative arguments to find a solution to the proposed application. On the other side, our idea is to obtain a result which could ensure the existence of a nontrivial critical point directly, without stressing either on geometry or on compactness of the associated functional, separately. Notwithstanding, it's worth pointing out clever abstract results obtained in [47, 48] (cf. Theorem 2.1 in both), where the authors also made use of "Monotonicity Trick" developed by L. Jeanjean in [27], for the purpose of getting critical points for a family of functionals, converging to a critical point the functional associated to the initial problem. These results have been applied to solve asymptotically linear problems with spectral properties similar to those presented in this paper, see [13], for instance.

In Chapter 2 are presented some initial applications for the abstract result. The first one has the aim of complementing the applications to Hamiltonian systems in [7], which to our knowledge, has not been tackled yet. More specifically, a periodic solution to an asymptotically 
quadratic Hamiltonian System is sought, whereas in [7] the authors present applications getting periodic solutions to a super-quadratic and a sub-quadratic Hamiltonian Systems. The following first order Hamiltonian System $(H S)$ is considered:

$$
\dot{z}=\mathscr{I} H_{z}(t, z), \quad z=(p, q) \in \mathbb{R}^{2 N},
$$

where $\mathscr{I}=\left(\begin{array}{cc}0 & -I \\ I & 0\end{array}\right)$ and $H \in C^{1}\left(\mathbb{R} \times \mathbb{R}^{2 N}, \mathbb{R}\right)$ has the form

$$
H(t, z)=\frac{1}{2} V(t) z \cdot z+R(t, z)
$$

Assuming that $V(t)$ satisfies

$\left(V_{0}\right) V(t)$ is continuous, $2 \pi$-periodic, symmetric $2 N \times 2 N$-matrix valued function.

Moreover, on $R(t, z)$ assuming the hypotheses

$\left(R_{1}\right) \quad R(t, z) \geq 0$, for all $z \in \mathbb{R}^{2 N}, t \in \mathbb{R}$ and $R_{z}(t, z)=o(|z|)$, uniformly in $t$ as $z \rightarrow 0$;

$\left(R_{2}\right) R_{z}(t, z)=M(t) z+r_{z}(t, z)$, with $M$ a continuous, $2 \pi$-periodic, symmetric $2 N \times 2 N$-matrix valued function and $r_{z}(t, z)=o(|z|)$, uniformly in $t$ as $|z| \rightarrow \infty$;

$\left(R_{3}\right)$ Setting $A$ as the operator of $L^{2}\left(S^{1}, \mathbb{R}^{2 N}\right)$ given by $A:=-\left(\mathscr{I} \frac{d}{d t}+V(t)\right)$,

$$
a_{0}:=\inf _{\substack{t \in \mathbb{R}, z \in \mathbb{R}^{2 N} \\|z|=1}}[M(t) z \cdot z]>\lambda^{+}=\inf [\sigma(A) \cap(0, \infty)]
$$

$\left(R_{4}\right)$ Defining $\mathscr{O}$ as the operator of $L^{2}\left(S^{1}, \mathbb{R}^{2 N}\right)$ given by $\mathscr{O}:=A-\mathscr{M}$, where $\mathscr{M}$ denotes the operator multiplication by $M(t), 0 \notin \sigma_{p}(\mathscr{O})$.

Other notable applications on first order Hamiltonian Systems in the same direction are the papers [49] and [15] where periodic solutions under $(C)_{c}$ condition are found. In [49] E. Silva considers a sub-quadratic Hamiltonian $H$ at infinity and, establishing a new version of an abstract saddle point theorem, he obtains a periodic solution complementing the application in [7]. On the other hand, D. Costa and C. Magalhães in [15] work with a non-quadratic Hamiltonian at infinity and assume either asymptotic non-crossing or crossing conditions. In comparison to their results, our assumptions generalize for the potential $V$, which is non-constant, although our hypotheses on $R$ are just slightly different from theirs on the non-quadraticity conditions.

The subsequently applications of Chapter 2 are on Schrödinger Equations. The first of them is for a problem corresponding to the Hamiltonian System treated previously, namely, 
problem $(P)$ below is considered

$$
-\Delta u+V(x) u=g(x, u) \text { in } \mathbb{R}^{N}, \quad N \geq 3
$$

in the case where analogous hypotheses to those under operator $A$ and nonlinearity $R$ in problem $(H S)$ are assumed. Hence, following the same lines as in problem $(H S)$ this problem is solved. In the subsequent section, problem $(P)$ is considered where $g(x, s)=h(x) f(s)$, and $h$ satisfies

$\left(h_{1}\right) h \in C\left(\mathbb{R}^{N},(0,+\infty)\right)$ and $\lim _{|x| \rightarrow+\infty} h(x)=0$;

$\left(h_{2}\right) h \in L^{q}\left(\mathbb{R}^{N}\right), q=\frac{2^{*}}{2^{*}-p}$, for some $p \in\left(2,2^{*}\right)$.

Furthermore, $f$ is asymptotically linear satisfying

$\left(f_{1}\right) \quad f \in C(\mathbb{R}, \mathbb{R})$ and $\lim _{s \rightarrow 0} \frac{f(s)}{s}=0 ;$

$\left(f_{2}\right)$ There exists $a>0$ such that $\lim _{s \rightarrow+\infty} \frac{F(s)}{s^{2}}=\frac{a}{2}$, where $F(s):=\int_{0}^{s} f(t) d t$, and $F(s) \geq 0$.

$\left(f_{3}\right)$ Setting $Q(s):=\frac{1}{2} f(s) s-F(s)>0$ for all $s \in \mathbb{R} \backslash\{0\}, \lim _{s \rightarrow+\infty} Q(s)=+\infty$.

Moreover, for the second application, is assumed that $V$ satisfies:

$\left(V_{1}\right) \quad V \in C\left(\mathbb{R}^{N}, \mathbb{R}\right)$ and $\lim _{|x| \rightarrow \infty} V(x)=V_{\infty}>0 ;$

$\left(V_{2}\right)$ Setting $A:=-\Delta+V(x)$, as an operator of $L^{2}\left(\mathbb{R}^{N}\right)$, and denoting by $\sigma(A)$ the spectrum of $A$,

$$
\sup [\sigma(A) \cap(-\infty, 0)]=\sigma^{-}<0<\sigma^{+}=\inf [\sigma(A) \cap(0,+\infty)] .
$$

For examples satisfying such hypotheses see pages 44 and 45 in this work.

This application was inspired by L. Maia, J. Oliveira Junior and R. Ruviaro [36], where they solved problem $(P)$ with potential $V$ satisfying $\left(V_{1}\right)-\left(V_{2}\right)$. In addition, they required that $0 \notin \sigma(A)$ and assumed some more hypotheses of decay and compactness on $V$. About the nonlinearity they set $h \equiv 1, f \in C^{3}\left(\mathbb{R}^{N}, \mathbb{R}\right)$, with some growth hypotheses on its derivatives, and assumed $f(s) / s$ being increasing as well. Since this kind of potential ensures that the subspace where $A$ is negative definite is finite dimensional, they could apply the aforementioned version of Linking Theorem introduced by $\mathrm{G}$. Li and $\mathrm{C}$. Wang in [34] to get $(C)_{c}$ sequence. They used the associated problem at infinity and a Splitting Lemma to compare the levels of both problems and get the necessary compactness. Trying to improve their result, our abstract linking theorem for $(C)_{c}$ sequences is applied and a nontrivial critical point is obtained straightway, avoiding such monotonicity assumptions on $f$.

Staring at our hypotheses, it is also possible to say that our second application complements the work by L. Jeanjean and K. Tanaka in [28]. In fact, they assumed $V(x) \geq \alpha>0$, and 
so they worked with Ekeland's principle to get a $(C)_{c}$ sequence and due to the geometry of their functional, they applied the Mountain Pass Theorem to get a critical point. They also worked with an asymptotically linear problem where $f(s) / s$ is not necessarily increasing. In addition, they assumed $h \equiv 1$ and $f(s) / s \rightarrow a>\inf \sigma(A)>0$ as $|s| \rightarrow+\infty$. Differently, in our case $V$ changes sign and $\inf \sigma(A)<0$, which implies a linking geometry and prevents us to use the same argument. However, considering the positive spectrum a similar hypothesis is assumed:

$$
a>\inf _{u_{1} \in E_{1}, u \neq 0} \frac{\int_{\mathbb{R}^{N}}\left(\left|\nabla u_{1}(x)\right|^{2}+V(x) u_{1}^{2}(x)\right) d x}{\int_{\mathbb{R}^{N}} h(x) u_{1}^{2}(x) d x} \geq \frac{1}{h_{\infty}} \inf [\sigma(A) \cap(0,+\infty)]=\frac{\sigma^{+}}{h_{\infty}}>0,
$$

where $\|h\|_{L^{\infty}\left(\mathbb{R}^{N}\right)}:=h_{\infty}$ and $E_{1}$ is the subspace of $H^{1}\left(\mathbb{R}^{N}\right)$ on which operator $A$ is positive definite. This hypothesis allows to develop a linking structure.

On the other hand, the work [17] by D. Costa and H. Tehrani can be cited, since the same $V$ as theirs is presented here. More specifically, they required $\left(V_{1}\right)$, and our hypothesis $\left(V_{2}\right)$ implies that either 0 is an isolated point of $\sigma(A)$, or it is in a gap of the spectrum, which is also required by them. However, they did not work with an asymptotically linear problem, but they assumed Ambrosetti-Rabinowitz well known condition, and required a nondecreasing nonlinearity. In their assumptions, $h=a$ is a sign-changing function in $C^{1}\left(\mathbb{R}^{N}, \mathbb{R}\right)$ and such that $\lim _{|x| \rightarrow+\infty} a(x)=a_{\infty}<0$, differently from $\lim _{|x| \rightarrow+\infty} h(x)=0$, in our case. Moreover, instead of using an abstract linking theorem, they applied a method of approximations to solve their problem.

It is also worth to mention the paper [22] by A. Edelson and C. Stuart, since assumptions close to theirs are considered here, however they asked $f(s) / s$ strictly increasing, which is removed here. Moreover, they applied the method of sub and super-solution and bifurcation to get a solution to their problem.

Finally, for the last application in Chapter 2 all assumptions on $h$ and $f$ are kept, as well as $\left(V_{2}\right)$, but assumption $\left(V_{1}\right)$ is replaced by the following:

$\left(V_{1}^{\prime}\right) V \in C\left(\mathbb{R}^{N}, \mathbb{R}\right)$ is $(2 \pi)$-periodic in $x_{i}, i=1, \ldots, N$

This application is motivated by the fact that in virtue of $\left(V_{1}\right)$, the subspace in which operator $A$ is negative definite, is finite dimensional. Since this is irrelevant for applying our abstract result, to solve a problem where both subspaces, in which operator $A$ is positive and negative definite, are infinite dimensional became our goal. In fact, $\left(V_{1}^{\prime}\right)$ combined with $\left(V_{2}\right)$ ensure the desired. Although all other hypotheses are kept, this replacement changes completely the spectral properties of $A$, which are fundamental to determine the linking geometry. It would be interesting to note that, the requirement of $V$ being a periodic function is done in order to explore spectral properties, hence a periodic nonlinearity is not required, since unlike most of the works in the literature (cf. [5, 31] for instance), the periodicity is not used to translate a $(C)_{c}$ 
sequence and ensure the existence of a critical point.

Chapter 3 handles the following radial nonlinear Schrödinger equation with a signchanging potential and an asymptotically linear nonlinearity

$$
-\Delta u+V(|x|) u=g(|x|, u) \text { in } \mathbb{R}^{N}, N \geq 3 .
$$

Our goal is to tackle the problem dropping off the monotonicity hypothesis on the nonlinear term, namely $g(x, s) / s$ nondecreasing on $s>0$ and loosen the regularity hypotheses on $g$ and $V$. In view of this, approaches as constrained minimization either on so the called Nehari or on the Generalized Nehari or Pohozaev Manifolds, applied in [2, 6, 22, 36, 53, 54, and references therein are discarded here. Instead, spectral properties of the Schrödinger operator $A:=-\Delta+V(|x|)$ are exploited with the purpose of getting the linking geometry for the indefinite functional associated to the elliptic equation in $\left(P_{r}\right)$. Since problem $\left(P_{r}\right)$ is radially symmetric, to deal with the Spectral Theory of $A$ restrictive hypotheses on $V$ are not necessary. In fact, it suffices to request informations under an associated operator $\bar{A}$ on the half-line, which is more manageable. Hence is assumed that the potential $V$ satisfies:

$\left(V_{1}\right)_{r} V \in L^{\infty}\left(\mathbb{R}^{N}\right)$ is a radial sign-changing function, $V(x)=V(|x|)=V(r), r \geq 0$;

$\left(V_{2}\right)_{r}$ Setting $\bar{V}(r)=V(r)+\frac{(N-1)(N-3)}{4 r^{2}}$ and $\bar{A}:=-\frac{d^{2}}{d r^{2}}+\bar{V}(r)$, an operator of $L^{2}(0,+\infty)$, $0 \notin \sigma_{\text {ess }}(\bar{A})$ and

$$
\sup [\sigma(\bar{A}) \cap(-\infty, 0)]=\sigma^{-}<0<\sigma^{+}=\inf [\sigma(\bar{A}) \cap(0,+\infty)] .
$$

Moreover, the nonlinearity $g$ is taken under the hypotheses:

$\left(g_{1}\right) g(x, s) \in C\left(\mathbb{R}^{N} \times \mathbb{R}, \mathbb{R}\right)$ is a radial function such that $\lim _{|s| \rightarrow 0} \frac{g(x, s)}{s}=0$, uniformly in $x$ and for all $t \in \mathbb{R}, G(x, t)=\int_{0}^{t} g(x, s) d s \geq 0$;

$\left(g_{2}\right) \lim _{|s| \rightarrow+\infty} \frac{g(x, s)}{s}=h(x)$, uniformly in $x$, where $h \in L^{\infty}\left(\mathbb{R}^{N}\right)$;

$\left(g_{3}\right) \quad a_{0}=\inf _{x \in \mathbb{R}^{N}} h(x)>\sigma^{+}=\inf [\sigma(A) \cap(0,+\infty)] ;$

$\left(g_{4}\right)$ Setting $\mathscr{O}:=A-\mathscr{H}$, where $\mathscr{H}$ is the operator multiplication by $h(x)$ in $L^{2}\left(\mathbb{R}^{N}\right)$ and denoting by $\sigma_{p}(\mathscr{O})$ the point spectrum of $\mathscr{O}, 0 \notin \sigma_{p}(\mathscr{O})$;

$\left(g_{5}\right)$ Defining $Q(x, s):=\frac{1}{2} g(x, s) s-G(x, s) \geq 0$ for all $(x, s) \in \mathbb{R}^{N} \times \mathbb{R}$ and $\sigma_{0}:=\min \left\{\sigma^{+},-\sigma^{-}\right\}$, there exists $\delta_{0}>0$ such that $\frac{g(x, s)}{s} \geq \sigma_{0}-\delta_{0} \Longrightarrow Q(x, s) \geq \delta_{0}$.

For examples satisfying theses assumptions see pages 66 and 67 in this text.

The inspiration for this work came from the papers [2,53]. In the former, A. Azzollini and A. Pomponio treated an autonomous radial nonlinear Schrödinger equation with a nonlinearity under Berestycki and Lions hypotheses (cf. [8]). Besides their potential $V \in C^{1}\left(\mathbb{R}^{N}, \mathbb{R}\right)$ satisfied 
some restrictions on its derivatives and $\lim _{|x| \rightarrow+\infty} V(x) \leq 0$, which ensured that $0 \in \sigma_{\text {ess }}(A)$ in their case. Hence, their work is complemented, considering cases such that $0 \notin \sigma_{\text {ess }}(A)$. Furthermore, here is only require $V \in L^{\infty}\left(\mathbb{R}^{N}\right)$ such that the spectrum of $A$ has a gap in 0 , which is at most an isolated point of $\sigma(A)$.

C. A. Stuart and H. S. Zhou in [53] worked with a class of radial nonlinear Schrödinger equations depending on $\lambda$, which is a constant potential and an asymptotically linear nonlinearity, but including the monotonicity hypothesis, as mentioned previously. They solved their class of problems by applying a variant of the Mountain Pass Theorem in [3]. The most interesting feature in their paper was to make use of the relation between the associated problem on the half-line and the original problem in $\mathbb{R}^{N}$. Following their ideas, spectral informations from the associated operator $\bar{A}$ on the half-line are extracted to guarantee that problem 3.1 satisfies the necessary conditions for the linking geometry.

Another notable work, which is worth mentioning is [56] by T. Watanabe. Although the author considers an autonomous radial nonlinear Schrödinger equation in $\mathbb{R}^{2}$, with positive potential and a nonlinearity with monotonicity assumption, our hypotheses are similar to theirs on the nonlinear term. Furthermore, as in [53] T. Watanabe first worked with the associated problem on the half-line, which also encouraged us to make the same.

Since the Hilbert space considered is $H_{r a d}^{1}\left(\mathbb{R}^{N}\right)$, the novelty lies in using this idea of investigating the spectral properties of the associated operator $\bar{A}$ on the half-line, and by doing this, avoiding a deeper study of the spectral theory of the operator $A$ in $H^{1}\left(\mathbb{R}^{N}\right)$. Thereby, it is possible to deal with much more general potentials, for instance those which do not have a limit at infinity.

Chapter 4 is devoted to solve non-cooperative elliptic systems under hypotheses on the potentials introduced by B. Sirakov in [50]. For $N \geq 2$ consider the elliptic system $(E S)$ given by

$$
\left\{\begin{aligned}
-\Delta u+V_{1}(x) u & =F_{u}(x, u, v) \quad \text { in } \quad \mathbb{R}^{N} \\
-\Delta v+V_{2}(x) v & =-F_{v}(x, u, v) \quad \text { in } \quad \mathbb{R}^{N},
\end{aligned}\right.
$$

where, for $i=1,2$, the potentials $V_{i} \in L_{l o c}^{\infty}\left(\mathbb{R}^{N}\right)$, satisfy

$\left(V_{1}\right)_{i}$ There exist constants $a_{i} \geq 0$ such that $V_{i}(x) \geq-a_{i}$ for all $x \in \mathbb{R}^{N}$;

$\left(V_{2}\right)_{i}$ Defining for all $\Omega \subset \mathbb{R}^{N}$ open,

$$
\nu_{i}(\Omega):=\inf \left\{\int_{\Omega}\left(|\nabla u(x)|^{2}+V_{i}(x) u^{2}(x)\right) d x: u \in H_{0}^{1}(\Omega),\|u\|_{L^{2}(\Omega)}=1\right\}
$$

assume that $\lim _{r \rightarrow+\infty} \nu_{i}\left(\mathbb{R}^{N} \backslash B_{r}\right)=+\infty$, where $B_{r}=\left\{x \in \mathbb{R}^{N}:|x| \leq r\right\}$; 
$\left(V_{3}\right)_{i}$ Setting the first eigenvalue of operator $A_{i}:=-\Delta+V_{i}(x)$ in $L^{2}\left(\mathbb{R}^{N}\right)$ by

$$
\lambda_{1}^{i}:=\inf \left\{\int_{\mathbb{R}^{N}}\left(|\nabla u(x)|^{2}+V_{i}(x) u^{2}(x)\right) d x: u \in H^{1}\left(\mathbb{R}^{N}\right),\|u\|_{L^{2}\left(\mathbb{R}^{N}\right)}=1\right\},
$$

assume that $\lambda_{1}^{i}>0$;

$(V)$ There exists $h(x) \in L_{l o c}^{\infty}\left(\mathbb{R}^{N}\right)$ and constants $\alpha_{0}>1, c_{0}>0, r_{0}>0$ such that

$$
h(x) \leq c_{0}\left[1+\left(\max _{i=1,2}\left\{0, V_{i}(x)\right\}\right)^{\frac{1}{\alpha_{0}}}\right], \quad \text { if } \quad|x| \geq r_{0} .
$$

Moreover, setting $a_{0}:=\inf _{x \in \mathbb{R}^{N}} h(x)$, assume that $a_{0}>\lambda_{1}^{1}>0$;

Once again inspired by [50], suitable hypotheses involving function $h(x)$ are required on $F$ for the both asymptotically and super quadratic cases. In fact, on the asymptotically quadratic case the following are required.

$\left(F_{1}\right) \quad F(x, s, t) \in C^{1}\left(\mathbb{R}^{N} \times \mathbb{R}^{2}, \mathbb{R}^{+}\right)$,

$$
\frac{F(x, s, t)}{h(x)}=o\left(|(s, t)|^{2}\right) \quad \text { as } \quad|(s, t)| \rightarrow 0, \text { and } \frac{F(x, s, t)}{|(s, t)|^{2}}=h(x)+o(1) \quad \text { as } \quad|(s, t)| \rightarrow+\infty,
$$

uniformly in $x \in \mathbb{R}^{N}$.

$\left(F_{2}\right)$ Setting $Q(x, s, t):=F_{s}(x, s, t) s+F_{t}(x, s, t) t-2 F(x, s, t)$, one has

$$
\lim _{|(s, t)| \rightarrow+\infty} Q(x, s, t)=+\infty, \text { uniformly in } x \in \mathbb{R}^{N} .
$$

On the other hand, the following assumptions are required for the super quadratic case.

$\left(F_{1}^{\prime}\right) \quad F(x, s, t) \in C^{1}\left(\mathbb{R}^{N} \times \mathbb{R}^{2}, \mathbb{R}^{+}\right)$,

$$
\frac{\left|\left(F_{s}(x, s, t), F_{t}(x, s, t)\right)\right|}{h(x)}=o(|(s, t)|) \quad \text { as } \quad|(s, t)| \rightarrow 0, \quad \text { uniformly in } \quad x,
$$

and for some $2<p<+\infty$ if $N=2$ or $2<p<2^{\#}:=\frac{4}{\alpha_{0}(N-2)}$ if $N>2$,

$$
\left|\left(F_{s}(x, s, t), F_{t}(x, s, t)\right)\right| \leq c_{0} h(x)\left(1+|(s, t)|^{p-1}\right), \quad \text { for } \quad \text { all } \quad(x, s, t) \in \mathbb{R}^{N} \times \mathbb{R}^{2}
$$

$\left(F_{2}^{\prime}\right) \lim _{|(s, t)| \rightarrow+\infty} \frac{F(x, s, t)}{|(s, t)|^{2}}=+\infty$, uniformly in $x$. 
In addition, defining $Q(x, u, v)=F_{u}(x, u, v) u+F_{v}(x, u, v) v-2 F(x, u, v)$ for all $(x, u, v) \in$ $\mathbb{R}^{N} \times \mathbb{R}^{2}$, in order to ensure the boundedness of Cerami sequences, the following condition is required.

$\left(F_{3}^{\prime}\right)$ There exist constants $c_{1}>0, \theta>\min \left\{1, \frac{N}{2}(p-2)\right\}$ such that for all $(x, u, v) \in \mathbb{R}^{N} \times \mathbb{R}^{2}$,

$$
Q(x, u, v) \geq c_{1} h(x)|(u, v)|^{\theta}
$$

or

$\left(F_{3}^{\prime \prime}\right)$ There exists a constant $D \geq 1$ such that

$$
Q(x, w, z) \leq D Q(x, u, v)
$$

for all $(u, v),(w, z) \in \mathbb{R}^{2}$ with $|(w, z)| \leq|(u, v)|$.

Examples that fit into these hypotheses are found in pages 80, 82 and 92 of this work.

A relevant motivation to study problem $(E S)$ stems from the system

$$
\left\{\begin{array}{l}
i \frac{\partial \Phi_{1}}{\partial t}=\Delta \Phi_{1}-V_{1}(x) \Phi_{1}-\mu_{1}\left|\Phi_{1}\right|^{2} \Phi_{1}-\beta_{12}\left|\Phi_{2}\right|^{2} \Phi_{1} \\
i \frac{\partial \Phi_{2}}{\partial t}=\Delta \Phi_{2}-V_{2}(x) \Phi_{2}-\mu_{2}\left|\Phi_{2}\right|^{2} \Phi_{2}-\beta_{21}\left|\Phi_{1}\right|^{2} \Phi_{2}
\end{array}\right.
$$

which has been used to model Bose-Einstein condensates in two different hyperfine spin states with the corresponding condensate wave functions $\Phi_{j}, j=1,2$. In this case, $V_{j}$ is the magnetic trapping potential for the respective hyperfine spin state, the constants $\mu_{j}$ and $\beta_{j k}$ are the intraspecies and interspecies scattering lengths, which represent the interactions between particles. If $\beta_{12}>0$ it means there is repulsive interaction between particles 1 and 2 , on the other hand, if $\beta_{12}<0$, there exists attractive interaction between them. In purpose of finding its solitary wave solutions of the form $\Phi_{j}=e^{-i \lambda_{j} t} u_{j}(x), \lambda_{j}>0$, for $j=1,2$, the system above is transformed into the elliptic system

$$
\left\{\begin{array}{l}
-\Delta u_{1}+\left(V_{1}(x)+\lambda_{1}\right) u_{1}+\mu_{1} u_{1}^{3}+\beta_{12} u_{2}^{2} u_{1}=0 \\
-\Delta u_{2}+\left(V_{2}(x)+\lambda_{2}\right) u_{2}+\mu_{2} u_{2}^{3}+\beta_{21} u_{1}^{2} u_{2}=0
\end{array}\right.
$$

Assuming $\beta_{12} \beta_{21}<0$ this elliptic system is non-cooperative and therefore it is a practical example of our problem. For details of physical applications see [12, 34] and references therein.

Originally, the motivation to study $(E S)$ came from [14, 16]. Indeed, in [14] D. G. Costa treated the cooperative elliptic system

$$
\left\{\begin{array}{l}
-\Delta u+a(x) u=F_{u}(x, u, v) \quad \text { in } \quad \mathbb{R}^{N} \\
-\Delta v+b(x) v=F_{v}(x, u, v) \quad \text { in } \quad \mathbb{R}^{N},
\end{array}\right.
$$


under the hypotheses that $a(x), b(x) \rightarrow+\infty$ as $n \rightarrow+\infty$ and the nonlinear term $F$ is nonquadratic at infinity. Moreover, he mentioned that his results could be generalized to noncooperative elliptic systems applying techniques found in [16]. Following this spirit and trying to generalize the results in [14] for non-cooperative elliptic systems, the remarkable paper [50], where B. Sirakov considered a scalar problem under general hypotheses on the potentials is recalled.

To the best of our knowledge, non-cooperative elliptic systems under assumptions similar to those introduced by B. Sirakov have not been treated yet. In fact, most of the well known papers in non-cooperative elliptic systems deals with constant potentials or even considers only bounded domains, as for instance, [4, 16, 19, 41] and references therein. Although there exists in the literature some relevant works on non-cooperative elliptic systems with non-constant potentials and treated on the whole space, normally their authors consider $V=V_{i}, i=1,2$, and assume hypotheses away from those required by us, check for example [24, 58].

In our point of view, the most interesting feature of hypotheses on $V_{i}$ is that the elliptic operator associated to problem $(E S)$ has pure point spectrum. Although such a spectrum has negative and positive parts composed by unbounded sequences, which makes the problem strongly indefinite, its discreteness brings the necessary compactness for the problem. Furthermore, the narrow relation between potentials $V_{i}$ and nonlinearity $F$, enables us to get the desired geometry for the problem. In view of these facts, it is feasible to find a non trivial solution to $(E S)$.

All hypotheses and observations made up to now, will be assumed throughout Chapter 4, however, asymptotically and super quadratic cases are going to be treated separately in Section 4.1 and 4.2 , respectively. In the super quadratic case, it is worth to focus in the boundedness of Cerami sequences, since the well known Ambrosetti-Rabinowitz condition [1] would not be sufficient as happens in the scalar problem. So, to circumvent this difficulty the resourceful hypothesis $(f 5)$ introduced by L. Jeanjean and K. Tanaka in [29] is assumed. Finally, it is important to point out that this scheme can be also applied to systems with more than two equations.

Finally, at the end of this work, a brief chapter stating auxiliary results is presented in order to make the reading easier. 


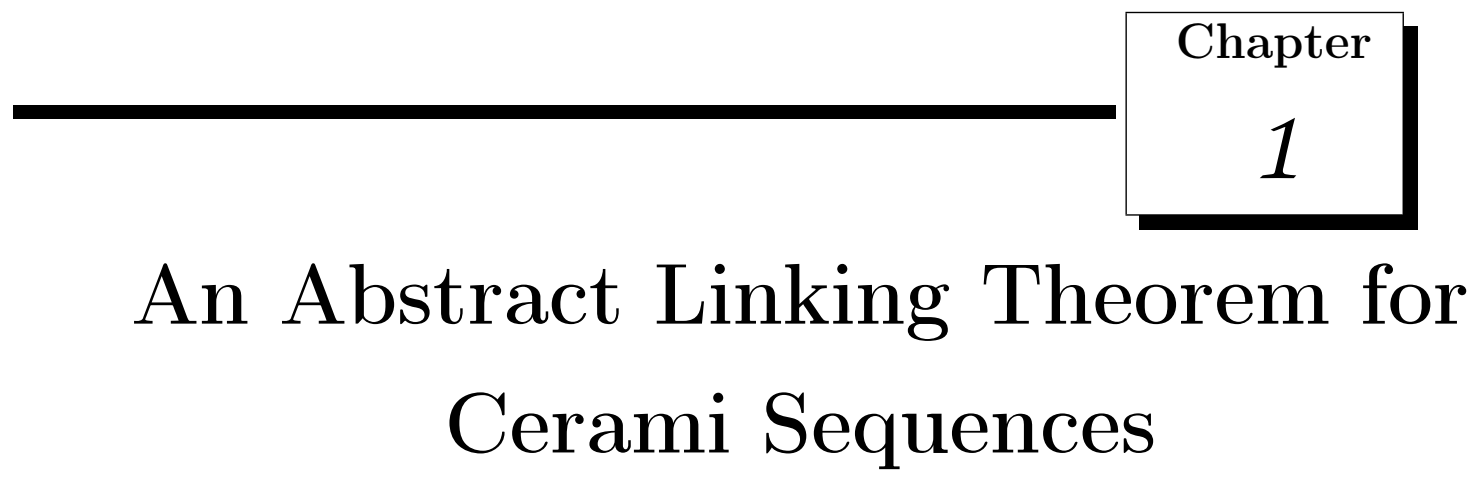

This chapter aims to develop an abstract critical point theorem for Cerami sequences by means of linking arguments, which will be applied in the next chapters to obtain solutions for indefinite problems involving nonlinear Schrödinger equations and Hamiltonian systems.

\subsection{Notion of Linking and Some Definitions}

In this section the work developed by Benci and Rabinowitz [7] is revisited, in order to present the notion of linking, and prove a new version of Abstract Linking Theorem based on their techniques, however working with Cerami sequences. Throughout this section $E$ always denotes a Hilbert space, $E=E_{1} \oplus E_{2}$ and if $u \in E$, write $u=u_{1}+u_{2}$ with $u_{i} \in E_{i}, i=1,2$, then set $P_{i} u:=u_{i}$, where $P_{i}: E \rightarrow E_{i}$ is the projector on $E_{i}, i=1,2$. Furthermore, mappings $h:[0,1] \times E \rightarrow E$ will be denoted by $h_{t}(u)$, and the closed ball of $E$ centered in zero with radius $r$, will be denoted by $B_{r}$. Furthermore, let $\mathscr{B}_{\tau}=\left(B_{\tau} \cap E_{1}\right) \oplus\left(B_{\tau} \cap E_{2}\right)$.

Definition 1.1. (Cf. [7]) Let $\Sigma$ denote the class of mappings $\Phi \in C([0,1] \times E, E)$, for which $P_{2} \Phi_{t}(u)=u_{2}-W_{t}(u)$, with $W_{t}$ compact for $t \in[0,1]$ and $\Phi_{0}(u)=u$. Let $S$ and $Q$ be Hilbert manifolds, $Q$ having a boundary $\partial Q, S$ and $\partial Q$ "link" if whenever $\Phi \in \Sigma$ and $\Phi_{t}(\partial Q) \cap S=\emptyset$, for all $t \in[0,1]$, then $\Phi_{t}(Q) \cap S \neq \emptyset$, for all $t \in[0,1]$.

Remark 1.1. A geometric understanding of this definition is that $S$ and $\partial Q$ link if every Hilbert manifold modeled on $Q$ and sharing the same boundary intersects $S$ (cf. [7]).

An useful example of linking sets, is provided in [7] and stated below. 
Lemma 1.1. (Cf. [7] Lemma 1.3) Let $e \in \partial B_{1} \cap E_{1}$ and $r_{1}>\rho>0$. If $S=\partial B_{\rho} \cap E_{1}$ and $Q=\left\{r e: r \in\left[0, r_{1}\right]\right\} \oplus\left(B_{r_{2}} \cap E_{2}\right)$, then $S$ and $Q$ link.

Proof. Let $\Phi \in \Sigma$ and suppose that $\Phi_{t}(\partial Q) \cap S=\emptyset$ for all $t \in[0,1]$. Is necessary to show that $\Phi_{t}(Q) \cap S \neq \emptyset$ or equivalently $P_{2} \Phi_{t}\left(q_{t}\right)=0$ and $\left\|P_{1} \Phi_{t}\left(q_{t}\right)\right\|=\left\|\Phi_{t}\left(q_{t}\right)\right\|=\rho$, for some $q_{t} \in Q$ and each $t \in[0,1]$. Let $r e+u \in Q$, and set

$$
\Psi(r e+u):=\left(\left\|P_{1} \Phi_{t}(r e+u)\right\|-\rho, P_{2} \Phi_{t}(r e+u)\right) .
$$

Then, observe that $P_{2} \Phi_{t}(r e+u)=u-W_{t}(r e+u)$ has the appropriate form to apply the theory of degree of Leray-Schauder. Denote the Leray-Schauder degree of a map $\Psi$ with respect to a bounded open set $\Omega$ and a point $a \notin \Psi(\partial Q)$ by $d(\Psi, \Omega, a)$. Consider $d\left(\Psi_{t}, Q, 0\right)$, since $\Phi(\partial Q) \cap S=\emptyset$, it is well defined for all $t \in[0,1]$. In fact, $0 \notin \Psi_{t}(\partial Q)$ for all $t \in[0,1]$, otherwise there would be $q_{t} \in \partial Q$ such that $\left\|P_{1} \Phi_{t}\left(q_{t}\right)\right\|=\rho$ and $P_{2} \Phi_{t}\left(q_{t}\right)=0$, and consequently $q_{t} \in S$, which is a contradiction. Thus, the homotopy invariance property of degree yields $d\left(\Psi_{t}, Q, 0\right)=d\left(\Psi_{0}, Q, 0\right)$ for all $t \in[0,1]$. Provided that $\Psi_{0}(r e+u)=(r-\rho, u)=0$, iff $r e+u=\rho e+0 \in Q$, then by the definition of degree $d\left(\Psi_{0}, Q, 0\right)=1$. Hence, $d\left(\Psi_{t}, Q, 0\right)=1$ for all $t \in[0,1]$, and thus, there exists $q_{t} \in Q$ such that $\Psi_{t}\left(q_{t}\right)=0$, namely $q_{t} \in \Phi_{t}(Q) \cap S$, and therefore, $\Phi_{t}(Q) \cap S \neq \emptyset$.

Henceforth, some definitions and notations introduced in [7] are required.

Definition 1.2. Let $B: E \rightarrow \mathbb{R}$ be a functional. $B$ is said to be uniformly differentiable in bounded subsets of $E$, if for any $R, \varepsilon>0$, there exists $\delta=\delta(R, \varepsilon)>0$, independent of $u$, such that

$$
\left|B(u+v)-B(u)-B^{\prime}(u) v\right| \leq \varepsilon|| v||
$$

for all $u, u+v \in B_{R}$ and $\|v\| \leq \delta$.

For the purpose of proving the abstract linking result in this chapter, next lemma is essential.

Lemma 1.2. Let $B: E \rightarrow \mathbb{R}$ be a functional which is weakly continuous and uniformly differentiable in bounded subsets of $E$. Then $B^{\prime}: E \rightarrow E^{\prime}$ is completely continuous.

Proof. Let $\left(u_{n}\right) \in E$ be a sequence such that $u_{n} \rightarrow u$ in $E$, then $\left(u_{n}\right)$ is bounded and there is $R>0$ such that $\left(u_{n}\right) \subset B_{R}$ and $u \in B_{R}$. Since $B$ is uniformly differentiable in bounded subsets of $E$, given $\varepsilon>0$ there exist $\delta>0$ such that

$$
\left|B(u+v)-B(u)-B^{\prime}(u) v\right| \leq \varepsilon|| v \|
$$

and

$$
\left|B\left(u_{n}+v\right)-B\left(u_{n}\right)-B^{\prime}\left(u_{n}\right) v\right| \leq \varepsilon\|v\|,
$$


for all $u+v, u_{n}+v \in B_{R}$ and $\|v\| \leq \delta$. Hence,

$$
\begin{aligned}
\left|\left(B^{\prime}\left(u_{n}\right)-B^{\prime}(u)\right) v\right| & \leq\left|B^{\prime}\left(u_{n}\right) v-\left(B\left(u_{n}+v\right)-B\left(u_{n}\right)\right)\right|+\left|(B(u+v)-B(u))-B^{\prime}(u) v\right| \\
& +\left|B\left(u_{n}+v\right)-B(u+v)\right|+\left|B(u)-B\left(u_{n}\right)\right| \\
& \leq 2 \varepsilon\|v\|+o_{n}(1)|| v \|
\end{aligned}
$$

as $n \rightarrow+\infty$, since $B$ is weakly continuous. Then,

$$
\left\|B^{\prime}\left(u_{n}\right)-B^{\prime}(u)\right\| \leq 2 \varepsilon+o_{n}(1)
$$

as $n \rightarrow+\infty$. Passing to the limit as $\varepsilon \rightarrow 0^{+}$, it yields $B^{\prime}\left(u_{n}\right) \rightarrow B^{\prime}(u)$ as $n \rightarrow+\infty$. Thus $B^{\prime}$ is completely continuous.

Definition 1.3. Let $\Gamma$ denote the set of mappings $h \in C([0,1] \times E, E)$ satisfying:

$\left(\Gamma_{1}\right) h_{t}(u)=U_{t}(u)+K_{t}(u)$, where $U, K \in C([0,1] \times E, E), U_{t}$ is a homeomorphism of $E$ onto $E$ and $K_{t}$ is compact for each $t \in[0,1]$;

$\left(\Gamma_{2}\right) \quad U_{0}(u)=u, K_{0}(u)=0$;

$\left(\Gamma_{3}\right) \quad P_{i} U_{t}(u)=U_{t}\left(P_{i}(u)\right), i=1,2$;

$\left(\Gamma_{4}\right) h_{t}$ maps bounded sets to bounded sets.

In addition, for $h \in \Gamma$, let $h_{t}^{j}(u)$ denote the $j$-fold composite of $h$ with itself, namely, $h_{t}^{1}(u)=h_{t}(u)$, $h_{t}^{2}(u)=h_{t}\left(h_{t}(u)\right)$, and $h_{t}^{j}(u)=h_{t}\left(h_{t}^{j-1}(u)\right)$, for $j>1$.

Now, it is convenient to state the abstract critical point theorem for Cerami sequences.

Theorem 1.4. (Abstract Linking Theorem) Let E be a real Hilbert space, with inner product $(\cdot, \cdot), E_{1}$ a closed subspace of $E$ and $E_{2}=E_{1}^{\perp}$. Let $I \in C^{1}(E, \mathbb{R})$ satisfying:

$\left(I_{1}\right) \quad I(u)=\frac{1}{2}(L u, u)+B(u)$, for all $u \in E$, where $u=u_{1}+u_{2} \in E_{1} \oplus E_{2}, L u=L_{1} u_{1}+L_{2} u_{2}$ and $L_{i}: E_{i} \rightarrow E_{i}, i=1,2$ is a bounded linear self adjoint mapping.

$\left(I_{2}\right) \quad B$ is weakly continuous and uniformly differentiable on bounded subsets of $E$.

$\left(I_{3}\right)$ There exist Hilbert manifolds $S, Q \subset E$, such that $Q$ is bounded and has boundary $\partial Q$, constants $\alpha>\omega$ and $v \in E_{2}$ such that

(i) $S \subset v+E_{1}$ and $I \geq \alpha$ on $S$;

(ii) $I \leq \omega$ on $\partial Q$;

(iii) $S$ and $\partial Q$ link, that is satisfy the linking Definition 1.1.

( $\left.I_{4}\right)$ Setting

$$
c:=\inf _{h \in \Lambda} \sup _{u \in \bar{Q}} I\left(h_{1}(u)\right)
$$


where $\bar{Q}$ is the closure of $Q$,

$$
\Lambda:=\left\{h \in C([0,1] \times E, E): \begin{array}{l}
h=h^{(1)} \circ \cdots \circ h^{(m)}, \quad h^{(1)}, \ldots, h^{(m)} \in \Gamma, m \in \mathbb{N} \\
h_{t}(\partial Q) \subset I^{\frac{\alpha-\omega}{2}}-\beta, \quad \beta \in\left(0, \frac{\alpha-\omega}{2}\right)
\end{array}\right\},
$$

and $I^{\lambda}=\{u \in E: I(u) \leq \lambda\}$, for all $\lambda \in \mathbb{R}$. If for a sequence $\left(u_{n}\right)$, there exists a constant $b>0$ such that $\left(u_{n}\right) \subset I^{-1}([c-b, c+b])$ and $\left(1+\left\|u_{n}\right\|\right)\left\|I^{\prime}\left(u_{n}\right)\right\| \rightarrow 0$ as $n \rightarrow+\infty$, then $\left(u_{n}\right)$ is bounded.

Then $c \geq \alpha$, and $c$ is a critical value of $I$.

Definition 1.5. Let $I \in C^{1}(E, \mathbb{R})$ be a functional. A sequence $\left(u_{n}\right) \subset E$ is said to be a Cerami sequence or a $(C)$ sequence for short, if it satisfies

$$
\sup _{n}\left|I\left(u_{n}\right)\right|<+\infty \text { and }\left\|I^{\prime}\left(u_{n}\right)\right\|\left(1+\left\|u_{n}\right\|\right) \rightarrow 0
$$

as $n \rightarrow+\infty$. Given $c \in \mathbb{R},\left(u_{n}\right) \subset E$ is said to be a Cerami sequence on level c or a $(C)_{c}$ sequence for short, if it satisfies

$$
I\left(u_{n}\right) \rightarrow c \text { and }\left\|I^{\prime}\left(u_{n}\right)\right\|\left(1+\left\|u_{n}\right\|\right) \rightarrow 0
$$

as $n \rightarrow+\infty$. Furthermore, functional I is said to satisfies Cerami condition or $(C)$ condition for short, if every Cerami sequence to I has convergent subsequence, analogously for $(C)_{c}$ condition.

\subsection{A Quantitative Deformation Lemma}

Inspired by [7] it is suitable to state a variant of the standard Quantitative Deformation Lemma for Cerami sequences, without Cerami condition, which is necessary to prove Theorem 1.4

Lemma 1.3. (Deformation Lemma): Let $I \in C^{1}(E, \mathbb{R})$ satisfying $\left(I_{1}\right)-\left(I_{2}\right)$ as in Theorem 1.4. Then for any $R \in \mathbb{N}, \varrho>0$ and $\varepsilon \in\left(0, \frac{1}{10}\right)$, if $s:=(R+2)^{2}$, there exist $k \in \mathbb{N}$ and $\eta \in \Gamma$, such that:

i) $I\left(\eta_{t}^{k s}(u)\right) \leq I(u)+\varrho$, for all $u \in \mathscr{B}_{R+2}$ and $t \in[0,1]$;

ii) If $c \in \mathbb{R}$ and $\left\|I^{\prime}(w)\right\|(1+\|w\|) \geq \sqrt{2 \varepsilon}$, for all $w \in \mathscr{B}_{R+1} \cap I^{-1}([c-\varepsilon, c+\varepsilon])$, then $I\left(\eta^{k s}(u)\right) \leq c-\frac{\varepsilon}{2}$, whenever $u \in \mathscr{B}_{\frac{R}{2}} \cap I^{-1}([c-\varepsilon, c+\varepsilon])$.

Remark 1.2. The mapping $\eta$ is usually determined by solving an appropriate differential equation involving $I^{\prime}(\eta)$. Such an approach seems to fail here since it does not give an $\eta$ satisfying $\left(\Gamma_{1}\right)-\left(\Gamma_{3}\right)$ which are crucial for the purpose of proving Theorem 1.4. Hence, in order to prove Lemma 1.3 it is necessary to argue similarly to Theorem 1.5 in [7]. 
Proof. First, choose $\chi \in C^{\infty}(\mathbb{R}, \mathbb{R})$ such that $\chi(t)=1$ for $t \leq R+1, \chi(t)=0$ for $t \geq R+2$, $\chi^{\prime}(t)<0$ for $t \in(R+1, R+2)$, and also assume $\chi(t) \leq(R+2-t)^{2}$ for $t \in\left[R+\frac{3}{2}, R+2\right]$. For $u=u_{1}+u_{2} \in E_{1} \oplus E_{2}$, set $V_{i}(u):=\chi\left(\left\|u_{i}\right\|\right) P_{i} I^{\prime}(u), i=1,2$ and $V(u):=V_{1}(u)+V_{2}(u)$. Note that $V(u) \equiv I^{\prime}(u)$ in $\mathscr{B}_{R+1}$. Moreover, by $\left(I_{1}\right)-\left(I_{2}\right)$, there is a constant $M=M(R)$ such that $\left\|I^{\prime}(u)\right\| \leq M$, for $u \in \mathscr{B}_{R+2}$. In fact, since $B$ is weakly continuous, it is bounded on $\mathscr{B}_{R+3}$, which is weakly compact in $E$. Let $M_{0}>0$ be a constant such that $|B(u)| \leq M_{0}$ for all $u \in \mathscr{B}_{R+3}$. Provided that $B$ is uniformly differentiable in $\mathscr{B}_{R+3}$, fixed $\varepsilon=1$, there exist $\delta_{0}>0$ such that $\left|B^{\prime}(u) v\right| \leq\|v\|+2 M_{0} \leq \delta_{0}+2 M_{0}$ for all $u+v, u \in \mathscr{B}_{R+3}$ and $\|v\| \leq \delta_{0}$. If $\delta_{0}>1$, for all $u \in \mathscr{B}_{R+2}$ and $\|v\| \leq 1$, it follows that $u+v \in \mathscr{B}_{R+3}$, then $\left|B^{\prime}(u) v\right| \leq \delta_{0}+2 M_{0}$. If $\delta_{0} \leq 1$, for all $\|v\| \leq 1$, note that $\left\|\frac{\delta_{0}}{\|v\|} v\right\|=\delta_{0} \leq 1$, hence for all $u \in \mathscr{B}_{R+2}$ it follows that $u+\frac{\delta_{0}}{\|v\|} v \in \mathscr{B}_{R+3}$ and then

$$
\left|B^{\prime}(u) v\right| \leq\left(\delta_{0}+2 M_{0}\right) \frac{\|v\|}{\delta_{0}} \leq \frac{1}{\delta_{0}}\left(\delta_{0}+2 M_{0}\right) .
$$

Thus, in both cases $\left\|B^{\prime}(u)\right\| \leq\left(1+\frac{1}{\delta_{0}}\right)\left(\delta_{0}+2 M_{0}\right)$. Therefore,

$$
\begin{aligned}
\left\|I^{\prime}(u)\right\| & =\left\|L u+B^{\prime}(u)\right\| \\
& \leq\|L\|\|u\|+\left\|B^{\prime}(u)\right\| \\
& \leq(R+2)\|L\|+\left(1+\frac{1}{\delta_{0}}\right)\left(\delta_{0}+2 M_{0}\right) \\
& :=M .
\end{aligned}
$$

With this, set

$$
\bar{\varepsilon}:=\frac{1}{M s} \min \left(\varrho, \frac{\varepsilon}{2}\right) .
$$

Since $I$ is uniformly differentiable on bounded sets, there is a $\delta=\delta(\bar{\varepsilon}, R)>0$ such that

$$
\left|I(u+v)-I(u)-I^{\prime}(u) v\right| \leq \bar{\varepsilon}|| v||
$$

for $u, u+v \in \mathscr{B}_{R+2}$ and $\|v\| \leq \delta$. Assume $\delta \leq 1$ and choose $k \in \mathbb{N}$ such that

$$
\frac{1}{k}<\min \left(\frac{\delta}{2 M}, \frac{1}{8(R+2)\left(1+\max _{\mathbb{R}}\left|\chi^{\prime}(t)\right|\left(|| L_{1}||+|| L_{2}||\right)\right.}\right) .
$$

Now define $\eta_{t}(u):=u-\frac{t}{k} V(u)$.

Claim. $\eta \in \Gamma$.

Assuming this claim and postponing its proof, it is necessary to check that $\mathscr{B}_{R+2}$ is an invariant set for $\eta_{t}$, for the purpose of proving $(i)-(i i)$. In fact, for $u=u_{1}+u_{2} \in \mathscr{B}_{R+2}$, by the 
definitions of $\eta$ and $V$ it follows that

$$
\left\|P_{i} \eta_{t}(u)-u_{i}\right\|=\left\|\left(u_{i}-\frac{t}{k} V_{i}(u)\right)-u_{i}\right\|=\frac{t}{k}\left\|V_{i}(u)\right\| \leq \frac{M}{k} \chi\left(\left\|u_{i}\right\|\right) .
$$

Note that, for $u_{i} \in B_{R+\frac{3}{2}}$, the choice of $\chi$ implies that

$$
\frac{M}{k} \chi\left(\left\|u_{i}\right\|\right) \leq \frac{1}{2} \leq R+2-\left\|u_{i}\right\|
$$

via 1.5 , while for $\left\|u_{i}\right\| \geq R+\frac{3}{2}$, it follows that

$$
\frac{1}{2} \geq R+2-\left\|u_{i}\right\| \geq\left(R+2-\left\|u_{i}\right\|\right)^{2} \geq \frac{M}{k} \chi\left(\left\|u_{i}\right\|\right),
$$

by the choice of $\chi$ and $k$. Hence, the right hand side of $(1.6)$ does not exceed $R+2-\left\|u_{i}\right\|$, $i=1,2$, which implies $\mathscr{B}_{R+2}$ is invariant for $\eta_{t}$. Indeed, from 1.6$)$ and the triangular inequality, it yields

$$
\left\|P_{i} \eta_{t}(u)\right\| \leq \frac{M}{k} \chi\left(\left\|u_{i}\right\|\right)+\left\|u_{i}\right\| \leq R+2 .
$$

Since $\operatorname{dist}\left(\eta_{t}(u), \partial \mathscr{B}_{R+2}\right)$, the distance from $\eta_{t}(u)$ to $\partial \mathscr{B}_{R+2}$ satisfies

$$
\operatorname{dist}\left(\eta_{t}(u), \partial \mathscr{B}_{R+2}\right)=\min _{i=1,2}\left(R+2-\left\|P_{i}\left(\eta_{t}(u)\right)\right\|\right),
$$

thus (1.7) implies that $\eta_{t}(u) \in \mathscr{B}_{R+2}$.

To prove $i$ ), observe that by 1.5 and the definitions of $\eta, M$ and $k$, it follows that

$$
\left\|\eta_{t}(u)-u\right\|=\left\|-\frac{t}{k} V(u)\right\|=\frac{t}{k}\|V(u)\| \leq \frac{M}{k}<\delta,
$$

for all $u \in \mathscr{B}_{R+2}$. Hence, fixing $u \in \mathscr{B}_{R+2}$ and using 1.4 with $v=-\frac{t}{k} V(u)$, it yields

$$
I(u+v)=I\left(\eta_{t}(u)\right) \leq I(u)-\frac{t}{k} I^{\prime}(u) V(u)+\frac{\bar{\varepsilon} t}{k}\|V(u)\| .
$$

Since $E_{2}=E_{1}^{\perp}$, then $P_{1}\left(I^{\prime}(u)\right) \perp P_{2}\left(I^{\prime}(u)\right)$, and by the definition of $V$, it follows that

$$
I^{\prime}(u) V(u)=\chi\left(\left\|u_{1}\right\|\right)\left\|P_{1}\left(I^{\prime}(u)\right)\right\|^{2}+\chi\left(\left\|u_{2}\right\|\right)\left\|P_{2}\left(I^{\prime}(u)\right)\right\|^{2} \geq 0 .
$$

Thus, by (1.3), 1.8), 1.9), 1.10) and the definition of $\bar{\varepsilon}$, it follows that

$$
I\left(\eta_{t}(u)\right) \leq I(u)+\frac{\bar{\varepsilon} M}{k} \leq I(u)+\frac{\varrho}{M s} \frac{M}{k}=I(u)+\frac{\varrho}{k s} .
$$

Provided that $\mathscr{B}_{R+2}$ is invariant under $\eta_{t}, i$ ) holds by iterating (1.11) $k s$ times. In fact, iterating twice means using $\eta_{t}(u)$ instead of $u$ in (1.11), and subsequently using again (1.11), but for $u$, it 
yields

$$
I\left(\eta_{t}^{2}(u)\right)=I\left(\eta_{t}\left(\eta_{t}(u)\right)\right) \leq I\left(\eta_{t}(u)\right)+\frac{\varrho}{k s} \leq I(u)+\frac{2 \varrho}{k s} .
$$

Then, after $k s$ iterations, it yields

$$
I\left(\eta_{t}^{k s}(u)\right) \leq I(u)+\frac{k s \varrho}{k s} \leq I(u)+\varrho,
$$

and $i$ ) is proved.

In order to prove $i i)$, take $u \in \mathscr{B}_{\frac{R}{2}} \cap I^{-1}([c-\varepsilon, c+\varepsilon])$. Three cases are considered:

Case I: $\eta_{1}^{j}(u) \in \mathscr{B}_{R+1} \cap I^{-1}([c-\varepsilon, c+\varepsilon])$ for $1 \leq j \leq k s$. By definition, $V(u)=I^{\prime}(u)$ in $\mathscr{B}_{R+1}$, then fixing $j, 1 \leq j \leq k s$, the definition of $\eta_{1}$ yields

$$
\eta_{1}^{j}(u)-\eta_{1}^{j-1}(u)=-\frac{1}{k} V\left(\eta_{1}^{j-1}(u)\right)=-\frac{1}{k} I^{\prime}\left(\eta_{1}^{j-1}(u)\right) .
$$

Then, using 1.9 for $\eta_{1}^{j}(u)$ and $\eta_{1}^{j-1}(u)$ instead of $u+v$ and $u$, by the definition of $M$, and due to 1.3 , it yields

$$
I\left(\eta_{1}^{j}(u)\right)-I\left(\eta_{1}^{j-1}(u)\right) \leq-\frac{1}{k}\left\|I^{\prime}\left(\eta_{1}^{j-1}(u)\right)\right\|^{2}+\frac{1}{k} \bar{\varepsilon} M \leq-\frac{1}{k}\left\|I^{\prime}\left(\eta_{1}^{j-1}(u)\right)\right\|^{2}+\frac{\varepsilon}{2 k s} .
$$

Then, by the telescopic sum, and using 1.12 for all $1 \leq j \leq k s$, it follows that

$$
I\left(\eta_{1}^{k s}(u)\right)-I(u)=\sum_{j=1}^{k s}\left[I\left(\eta_{1}^{j}(u)\right)-I\left(\eta_{1}^{j-1}(u)\right)\right] \leq \sum_{j=1}^{k s}\left[-\frac{1}{k}\left\|I^{\prime}\left(\eta_{1}^{j-1}(u)\right)\right\|^{2}+\frac{\varepsilon}{2 k s}\right] .
$$

Provided that $(R+2)\left\|I^{\prime}(u)\right\| \geq(1+\|u\|)\left\|I^{\prime}(u)\right\| \geq \sqrt{2 \varepsilon}$ from assumption, setting $\varepsilon_{s}:=\frac{\varepsilon}{s}$, it follows that $\left\|I^{\prime}(u)\right\|^{2} \geq 2 \varepsilon_{s}$, for all $u \in \mathscr{B}_{R+1}$. Thus, 1.13 yields

$$
I\left(\eta_{1}^{k s}(u)\right)-I(u) \leq \sum_{j=1}^{k s}\left[-\frac{2 \varepsilon_{s}}{k}+\frac{\varepsilon_{s}}{2 k}\right]=-\frac{3 \varepsilon}{2} .
$$

Therefore, since $I(u) \leq c+\varepsilon, 1.14$ implies that $I\left(\eta_{1}^{k s}(u)\right) \leq I(u)-\frac{3 \varepsilon}{2} \leq c-\frac{\varepsilon}{2}$, and the result holds for first case.

Case II: $\eta_{1}^{j}(u) \in \mathscr{B}_{R+1} \cap I^{-1}([c-\varepsilon, c+\varepsilon])$ for $1 \leq j \leq m-1$, but $\eta_{1}^{m}(u) \notin I^{-1}([c-\varepsilon, c+\varepsilon])$, for some $1 \leq m \leq k s$. From 1.12 and since $\left\|I^{\prime}(u)\right\|^{2} \geq 2 \varepsilon_{s}$, for all $u \in \mathscr{B}_{R+1}$, it follows that

$$
I\left(\eta_{1}^{j}(u)\right)-I\left(\eta_{1}^{j-1}(u)\right) \leq-\frac{2 \varepsilon_{s}}{k}+\frac{\varepsilon_{s}}{2 k}=-\frac{3 \varepsilon_{s}}{2 k},
$$

hence, $I\left(\eta_{1}^{j}(u)\right)<I\left(\eta_{1}^{j-1}(u)\right)$ for $1 \leq j \leq m$. Then, $\eta_{1}^{m}(u) \notin I^{-1}([c-\varepsilon, c+\varepsilon])$ and 
$I\left(\eta_{1}^{m}(u)\right)<I\left(\eta_{1}^{m-1}(u)\right)$ implies that $I\left(\eta_{1}^{m}(u)\right)<c-\varepsilon$. Thus, using again a telescopic sum, it follows that

$$
\begin{aligned}
I\left(\eta_{1}^{k s}(u)\right) & =I\left(\eta_{1}^{m}(u)\right)+\sum_{j=m+1}^{k s}\left[I\left(\eta_{1}^{j}(u)\right)-I\left(\eta_{1}^{j-1}(u)\right)\right] \\
& \leq c-\varepsilon+\sum_{j=m+1}^{k s}\left[I\left(\eta_{1}^{j}(u)\right)-I\left(\eta_{1}^{j-1}(u)\right)\right] .
\end{aligned}
$$

Fixing $j, m+1 \leq j \leq k s$, and using 1.9 as in 1.12 , it yields

$$
I\left(\eta_{1}^{j}(u)\right)-I\left(\eta_{1}^{j-1}(u)\right) \leq-\frac{1}{k}\left\|I^{\prime}\left(\eta_{1}^{j-1}(u)\right)\right\|^{2}+\frac{\varepsilon}{2 k s} \leq \frac{\varepsilon}{2 k s} .
$$

Replacing (1.16) in (1.15) for all $m+1 \leq j \leq k s$, it follows that

$$
\begin{aligned}
I\left(\eta_{1}^{k s}(u)\right) & \leq c-\varepsilon+\sum_{j=m+1}^{k s}\left[\frac{\varepsilon}{2 k s}\right] \\
& =c-\varepsilon+\left(\frac{k s-m}{k s}\right) \frac{\varepsilon}{2} \\
& \leq c-\frac{\varepsilon}{2} .
\end{aligned}
$$

Therefore, the result also holds in this case.

Case III: $\eta_{1}^{j}(u) \in \mathscr{B}_{R+1} \cap I^{-1}([c-\varepsilon, c+\varepsilon])$ for $1 \leq j \leq m-1$, but $\eta_{1}^{m}(u) \notin \mathscr{B}_{R+1}$ for some $1 \leq m \leq k s$. Since $u \in \mathscr{B}_{\frac{R}{2}}$, it follows that

$$
\|u\|+\frac{R}{2}+1 \leq R+1 \leq\left\|\eta_{1}^{m}(u)\right\|
$$

and hence, by the triangular inequality and the telescopic sum, it follows that

$$
\begin{aligned}
\frac{R+2}{2} & \leq\left\|\eta_{1}^{m}(u)\right\|-\|u\| \\
& \leq\left\|\eta_{1}^{m}(u)-u\right\| \\
& \leq \sum_{j=1}^{m}\left\|\eta_{1}^{j}(u)-\eta_{1}^{j-1}(u)\right\| .
\end{aligned}
$$

By the definition of $\eta_{1}$, and that $V(u)=I^{\prime}(u)$ in $\mathscr{B}_{R+1}$, it follows that

$$
\left\|\eta_{1}^{j}(u)-\eta_{1}^{j-1}(u)\right\|=\frac{1}{k}\left\|V\left(\eta_{1}^{j-1}(u)\right)\right\|=\frac{1}{k}\left\|I^{\prime}\left(\eta_{1}^{j-1}(u)\right)\right\|,
$$

for all $1 \leq j \leq m$, since $\eta_{1}^{j}(u) \in \mathscr{B}_{R+1}$ for all $1 \leq j \leq m-1$. Hence, replacing 1.18 in 1.17 
and applying Hölder's Inequality for finite sums, it yields

$$
\begin{aligned}
\frac{R+2}{2} & \leq \frac{1}{k} \sum_{j=1}^{m}\left\|I^{\prime}\left(\eta_{1}^{j-1}(u)\right)\right\| \\
& \leq \frac{m^{\frac{1}{2}}}{k}\left[\sum_{j=1}^{m}\left\|I^{\prime}\left(\eta_{1}^{j-1}(u)\right)\right\|^{2}\right]^{\frac{1}{2}} .
\end{aligned}
$$

From 1.12 , it follows that $\frac{1}{k}\left\|I^{\prime}\left(\eta_{1}^{j-1}(u)\right)\right\|^{2} \leq-\left[I\left(\eta_{1}^{j}(u)\right)-I\left(\eta_{1}^{j-1}(u)\right)\right]+\frac{\varepsilon}{2 k s}$, which yields

$$
\left\|I^{\prime}\left(\eta_{1}^{j-1}(u)\right)\right\|^{2} \leq k\left(I\left(\eta_{1}^{j-1}(u)\right)-I\left(\eta_{1}^{j}(u)\right)\right)+\frac{\varepsilon_{s}}{2},
$$

for all $1 \leq j \leq m$. Thus, using 1.20 in 1.19 , it yields

$$
\frac{R+2}{2} \leq \frac{m^{\frac{1}{2}}}{k}\left[\sum_{j=1}^{m} k\left(I\left(\eta_{1}^{j-1}(u)\right)-I\left(\eta_{1}^{j}(u)\right)\right)+\frac{\varepsilon_{s}}{2}\right]^{\frac{1}{2}}=\frac{m^{\frac{1}{2}}}{k}\left[k\left(I(u)-I\left(\eta_{1}^{m}(u)\right)\right)+\frac{m \varepsilon_{s}}{2}\right]^{\frac{1}{2}} .
$$

Squaring both sides, it follows that

$$
\begin{aligned}
\frac{s}{4}=\left(\frac{R+2}{2}\right)^{2} & \leq \frac{m}{k^{2}}\left[k\left(I(u)-I\left(\eta_{1}^{m}(u)\right)\right)+\frac{m \varepsilon_{s}}{2}\right] \\
& =\frac{m}{k}\left[I(u)-I\left(\eta_{1}^{m}(u)\right)+\frac{m \varepsilon}{2 k s}\right] \\
& \leq \frac{m}{k}\left[c+\varepsilon-I\left(\eta_{1}^{m}(u)\right)+\frac{\varepsilon}{2}\right] .
\end{aligned}
$$

Multiplying both sides of 1.21 by $\frac{k}{m}$, it yields

$$
\frac{1}{4} \leq \frac{k s}{4 m} \leq c+\frac{3 \varepsilon}{2}-I\left(\eta_{1}^{m}(u)\right)
$$

which implies $I\left(\eta_{1}^{m}(u)\right) \leq c+\frac{3 \varepsilon}{2}-\frac{10 \varepsilon}{4}=c-\varepsilon$, since $0<\varepsilon<\frac{1}{10}$ by assumption. Now, as in Case $I I$, using a telescopic sum and (1.16), it yields

$$
\begin{aligned}
I\left(\eta_{1}^{k s}(u)\right) & =I\left(\eta_{1}^{m}(u)\right)+\sum_{j=m+1}^{k s}\left[I\left(\eta_{1}^{j}(u)\right)-I\left(\eta_{1}^{j-1}(u)\right)\right] \\
& \leq c-\varepsilon+\sum_{j=m+1}^{k s}\left[\frac{\varepsilon}{2 k s}\right] \\
& =c-\varepsilon+\left(\frac{k s-m}{k s}\right) \frac{\varepsilon}{2} \\
& \leq c-\frac{\varepsilon}{2}
\end{aligned}
$$


Therefore $i$ i) also holds for the third case.

Proof of the Claim: Finally, to finish the proof of this lemma, it is left to prove the claim. Denoting by $I d: E \rightarrow E$ the identity map in $E$, since

$$
P_{i} V(u)=V_{i}(u)=\chi\left(\| u_{i}||\right) P_{i} I^{\prime}(u)=\chi\left(\left\|u_{i}\right\|\right)\left(L_{i} u_{i}+P_{i} B^{\prime}(u)\right)
$$

it holds that

$$
P_{i} \eta_{t}(u)=P_{i}\left(u-\frac{t}{k} V(u)\right)=\left(I d-\frac{t}{k} \chi\left(\left\|u_{i}\right\|\right) L_{i}\right) u_{i}-\frac{t}{k} \chi\left(\left\|u_{i}\right\|\right) P_{i} B^{\prime}(u) .
$$

Hence, it is suitable to set

$$
U_{t}(u):=\sum_{i=1,2}\left(I d-\frac{t}{k} \chi\left(\left\|u_{i}\right\|\right) L_{i}\right) u_{i},
$$

and

$$
K_{t}(u):=-\frac{t}{k} \sum_{i=1,2} \chi\left(\left\|u_{i}\right\|\right) P_{i} B^{\prime}(u)
$$

In fact, observe that given $u_{n} \rightarrow u$ in $E,\left(I_{2}\right)$ and Lemma 1.2 imply that $B^{\prime}\left(u_{n}\right) \rightarrow B^{\prime}(u)$ in $E^{\prime}$. Since $P_{i}$ is continuous, it follows that $K_{t}\left(u_{n}\right) \rightarrow K_{t}(u)$, thus $K_{t}$ is completely continuous, and therefore is compact for all $t \in[0,1]$. Moreover, by the definitions of $U_{t}$ and $K_{t}$, it is clear that $\eta$ satisfies $\left(\Gamma_{2}\right)-\left(\Gamma_{3}\right)$. Via $\left(I_{1}\right)-\left(I_{2}\right)$ there is a constant $C=C(r)$ such that $\left\|I^{\prime}(u)\right\| \leq C$ for all $u \in B_{r}$, hence by the definition of $\eta_{t}$, it yields

$$
\begin{aligned}
\left\|\eta_{t}(u)\right\| & \leq\|u\|+\|V(u)\| \\
& \leq r+\left\|I^{\prime}(u)\right\| \\
& \leq r+C
\end{aligned}
$$

and $\eta_{t}$ maps bounded sets into bounded sets, namely $\eta$ satisfies $\left(\Gamma_{4}\right)$. Lastly, it suffices to show that $U_{t}$ is a homeomorphism of $E$ onto $E$, so as to complete the verification of $\left(\Gamma_{1}\right)$. By $\left(\Gamma_{3}\right)$, it suffices to show that $P_{i} U_{t}$ is a homeomorphism of $E_{i}$ onto $E_{i}, i=1,2$. Let $u, v \in E_{i}$, for any $t \in[0,1]$, by the definition of $\chi$, if $\|u\|,\|v\| \geq R+2$, then

$$
\frac{t}{k}\left\|\chi(\|u\|) L_{i} u-\chi(\|v\|) L_{i} v\right\|=0 \leq \frac{1}{2}\|u-v\| .
$$

Hence, without loss of generality, suppose that $\|v\| \leq R+2$. By the definitions of $\chi$ and $k$, and via Mean Value Theorem, for any $t \in[0,1]$, it yields 


$$
\begin{aligned}
\frac{t}{k}\left\|\chi(\|u\|) L_{i} u-\chi(\|v\|) L_{i} v\right\| & \leq \frac{1}{k}\left\|\chi(\|u\|)\left(L_{i} u-L_{i} v\right)\right\|+\frac{1}{k}\left\|(\chi(\|u\|)-\chi(\|v\|)) L_{i} v\right\| \\
& \leq \frac{1}{k}\left\|L_{i}\right\|\|u-v\|+\frac{1}{k}\left\|L_{i}\right\||\chi(\|u\|)-\chi(\|v\|)|\|v\| \\
& \leq \frac{1}{k}\left\|L_{i}\right\|\left[\|u-v\|+(R+2) \max _{\mathbb{R}}\left|\chi^{\prime}(t)\right| \mid\|u\|-\|v\| \|\right] \\
& \leq \frac{1}{k}\left\|L_{i}\right\|(R+2)\left[1+\max _{\mathbb{R}}\left|\chi^{\prime}(t)\right|\right]\|u-v\| \\
& \leq \frac{1}{2}\|u-v\| .
\end{aligned}
$$

Thus, 1.22 holds for all $u, v \in E_{i}$. Now, for each $w \in E_{i}$ fixed, set $\mathscr{L}_{w}: E_{i} \rightarrow E_{i}$, given by $\mathscr{L}_{w}(u):=\frac{t}{k} \chi(\|u\|) L_{i} u+w$. Note that due to $1.22, \mathscr{L}_{w}(u)$ is a contraction on $E_{i}$. Then, it follows from Contracting Mapping Theorem that $\mathscr{L}_{w}(u)$ has a unique fixed point $u_{w}$. Therefore, $u_{w} \in E_{i}$ is the unique such that $\mathscr{L}_{w}\left(u_{w}\right)=u_{w}$, which implies that $P_{i} U_{t}\left(u_{w}\right)=w$ is a one-to-one correspondence, and hence $P_{i} U_{t}$ is bijection. Furthermore, 1.22 yields

$$
\begin{aligned}
\left\|P_{i} U_{t}(u)-P_{i} U_{t}(v)\right\| & =\left\|(u-v)-\frac{t}{k}\left(\chi(\|u\|) L_{i} u-\chi(\|v\|) L_{i} v\right)\right\| \\
& \geq\|u-v\|-\frac{t}{k}\left\|\chi(\|u\|) L_{i} u-\chi(\|v\|) L_{i} v\right\| \\
& \geq\|u-v\|-\frac{1}{2}\|u-v\|=\frac{1}{2}\|u-v\|,
\end{aligned}
$$

which implies that $\left(P_{i} U_{t}\right)^{-1}$ is continuous. Since $P_{i} U_{t}$ is continuous by definition, it ensures that $P_{i} U_{t}$ is a homeomorphism of $E_{i}$ onto $E_{i}$. Consequently, $\eta$ satisfies $\left(\Gamma_{1}\right)$ and finally the claim is proved.

The following lemma gives a significant information about level $c$.

Lemma 1.4. (Cf. [7] Proposition 1.17.) If I satisfies $\left(I_{3}\right)$, then $c \geq \alpha$.

Proof. For sake of completeness, this proof is included here. In fact, it suffices to show that

$$
h_{1}(\bar{Q}) \cap S \neq \emptyset,
$$

for all $h \in \Lambda$. In fact, if 1.24 holds, there is a $y \in h_{1}(\bar{Q}) \cap S$, hence

$$
\sup _{u \in \bar{Q}} I\left(h_{1}(u)\right) \geq I(y) \geq \inf _{w \in S} I(w) \geq \alpha
$$

due to $\left(I_{3}\right)(i)$. Since 1.25 holds for all $h \in \Lambda$, the definition of $c$ in $\left(I_{4}\right)$ yields $c \geq \alpha$. The proof of 1.24 follows from the stronger assertion that

$$
h_{t}(\bar{Q}) \cap S \neq \emptyset,
$$


for all $h \in \Lambda$ and $t \in[0,1]$. Since $S-v \subset E_{1}$ via $\left(I_{3}\right)(i)$, then 1.26 is equivalent to finding, for each $t \in[0,1]$, a $u \in \bar{Q}$ such that

$$
\begin{aligned}
& P_{1} h_{t}(u) \in S-v \\
& P_{2} h_{t}(u)=v .
\end{aligned}
$$

In order to solve (1.27), it is necessary to convert it into an equivalent problem to which the linking geometry hypotheses can be applied. Suppose first that $h \in \Lambda$ with the corresponding $m=1$. Letting $u=u_{1}+u_{2} \in E_{1} \oplus E_{2}$ as usual, by $\left(\Gamma_{1}\right)$ and $\left(\Gamma_{3}\right), 1.27$ becomes

$$
\begin{aligned}
& \text { (i) } P_{1} h_{t}(u) \in S-v \\
& \text { (ii) } P_{2} h_{t}(u)=U_{t}\left(u_{2}\right)+P_{2} K_{t}(u)=v .
\end{aligned}
$$

More generally, suppose (1.28) (ii) replaced by

$$
P_{2} h_{t}(u)=P_{2} Z_{t}(u)
$$

where $Z_{t}(u)$ is an arbitrary compact operator with $Z_{0}(u)=v$. Note that in $1.28, Z_{t}(u)=v$ is the compact constant operator, for all $t \in[0,1]$. Again via $\left(\Gamma_{1}\right)$ and $\left(\Gamma_{3}\right)(1.29)$ is equivalent to

$$
U_{t}\left(u_{2}\right)=-P_{2}\left(K_{t}(u)-Z_{t}(u)\right)
$$

which is equivalent to

$$
u_{2}=U_{t}^{-1}\left(-P_{2}\left(K_{t}(u)-Z_{t}(u)\right)\right) \equiv P_{2} Y_{t}(u)
$$

where $Y_{t}$ is compact, due to the compactness of $K_{t}$ and $Z_{t}$, and $Y_{0}(u)=v$, since

$$
U_{0}^{-1}\left(-P_{2}\left(K_{0}(u)-Z_{0}(u)\right)\right)=-P_{2}\left(-Z_{0}(u)\right)=v .
$$

Now, suppose by induction that 1.29 is equivalent to

$$
u_{2}=P_{2} Y_{t}(u)
$$

with $Y_{t}$ compact and $Y_{0}(u)=v$, whenever $h \in \Lambda$ with the corresponding $m=n-1$. Then, let $h \in \Lambda$ with $m=n$ so $h=h^{(1)} \circ \cdots \circ h^{(m)}$ and let $\hat{h}=h^{(2)} \circ \cdots \circ h^{(m)}$. Hence $h=h^{(1)} \circ \hat{h}$ and again by $\left(\Gamma_{1}\right)$ and $\left(\Gamma_{3}\right)$, equation

$$
v=P_{2} h_{t}(u)=P_{2}\left(U_{t}^{(1)}+K_{t}^{(1)}\right) \hat{h}_{t}(u)=U_{t}^{(1)} P_{2} \hat{h}_{t}(u)+P_{2} K_{t}^{(1)} \hat{h}_{t}(u)
$$


is equivalent to

$$
P_{2} \hat{h}_{t}(u)=\left(U_{t}^{(1)}\right)^{-1}\left(-P_{2} K_{t}^{(1)}\left(\hat{h}_{t}(u)\right)+v\right)=: P_{2} \hat{Z}_{t}(u)
$$

where $h^{(1)}=U^{(1)}+K^{(1)}$ and since $K_{t}^{(1)}$ is compact, $\hat{Z}_{t}$ given by the right hand side of 1.32 is compact and $P_{2} \hat{Z}_{0}(u)=v$, since $K_{0}=0$. Thus, by induction hypothesis there is a compact $Y_{t}$ such that 1.32 is equivalent to solving (1.31).

Now set $\Phi_{t}(u)=P_{1} h_{t}(u)+u_{2}-P_{2} Y_{t}(u)+v$, and note that $\Phi \in \Sigma$, in Definition 1.1 . since

$$
P_{2} \Phi_{t}=u_{2}-\left(P_{2} Y_{t}-v\right)
$$

and

$$
\Phi_{0}(u)=P_{1} h_{0}(u)+u_{2}-P_{2} Y_{0}(u)+v=P_{1} u+u_{2}-v+v=u .
$$

In addition, $P_{1} \Phi_{t}=P_{1} h_{t}$ and provided that $P_{2} h_{t}=v$ is equivalent to (1.31), due to all remarks above, it follows that $P_{2} \Phi_{t}=v$ is equivalent to $P_{2} h_{t}=v$, by the definition of $\Phi_{t}$. Therefore, $\Phi_{t}(u) \in S$ if and only if $h_{t}(u) \in S$, hence to obtain 1.26 and complete the proof, it is only necessary to show that

$$
\Phi_{t}(Q) \cap S \neq \emptyset,
$$

for all $t \in[0,1]$. Since $\Phi \in \Sigma$ and via $\left(I_{3}\right)$ (iii), $S$ and $\partial Q$ link, then 1.33 holds if

$$
\Phi_{t}(\partial Q) \cap S=\emptyset
$$

Suppose the contrary, so there is a $u \in \partial Q$ and $t \in[0,1]$ such that $\Phi_{t}(u) \in S$. Then $h_{t}(u) \in S$, but $h_{t}(\partial Q) \subset I^{\frac{\alpha+\omega}{2}-\beta}$, since $h \in \Lambda$. On the other hand, $S \cap I^{\frac{\alpha+\omega}{2}-\beta}=\emptyset$, due to $\left(I_{3}\right)(i)$ and provided that $\beta \in\left(0, \frac{\alpha-\omega}{2}\right)$, hence it yields a contradiction. Thus 1.34 is satisfied and this proof is complete.

\subsection{Proof of the Main Result}

Under the knowledge of all results in previous sections, Theorem 1.4 can be finally proved.

Proof of Theorem 1.4. First, since identity map $h(u)=u$ is in $\Lambda$, then $c<+\infty$ in view of $\left(I_{2}\right)$ and $\left(I_{3}\right)$. Moreover, $c \geq \alpha$ by Lemma 1.4. Now suppose that $c$ is not a critical value of $I$, then $I^{\prime}(u) \neq 0$, for all $u \in I^{-1}(c)$, hence there exists $\varepsilon>0$ such that

$$
(1+\|u\|)\left\|I^{\prime}(u)\right\| \geq \sqrt{2 \varepsilon}, \text { for all } u \in I^{-1}([c-\varepsilon, c+\varepsilon]) .
$$

If not, there exists a sequence of positive $\varepsilon_{n} \rightarrow 0$ and $u_{n} \in I^{-1}\left(\left[c-\varepsilon_{n}, c+\varepsilon_{n}\right]\right)$ such that

$$
\left(1+\left\|u_{n}\right\|\right)\left\|I^{\prime}\left(u_{n}\right)\right\|<\sqrt{2 \varepsilon_{n}} .
$$


From $\left(I_{4}\right)$ this sequence is bounded and then it possesses a weakly convergent subsequence, still denoted by $\left(u_{n}\right)$, namely $u_{n} \rightarrow u$ as $n \rightarrow+\infty$, for some $u \in E$. By $\left(I_{2}\right)$ and Lemma 1.2 one has $B^{\prime}\left(u_{n}\right) \rightarrow B^{\prime}(u)$ along this subsequence and by assumption, $I^{\prime}\left(u_{n}\right) \rightarrow 0$, then it follows that $L u_{n}=I^{\prime}\left(u_{n}\right)-B^{\prime}\left(u_{n}\right) \rightarrow-B^{\prime}(u)$ as $n \rightarrow+\infty$. On the other hand, $L u_{n}$ also converges weakly to $L u$ along this subsequence. Hence $L u=-B^{\prime}(u)$ and $L u_{n} \rightarrow L u$ strongly, then $I^{\prime}(u)=L u+B^{\prime}(u)=0$. Since $I\left(u_{n}\right) \rightarrow c$, again by $\left(I_{2}\right)$ it follows that $I\left(u_{n}\right)=\frac{1}{2}\left(L u_{n}, u_{n}\right)+B\left(u_{n}\right) \rightarrow I(u)=c$. But it means that $c$ is a critical value of $I$, contrary to assumption. Thus there exists an $\varepsilon$ as desired in 1.35 . It can further be assumed $\varepsilon<\frac{1}{10}$. By the definition of infimum, choose an $h \in \Lambda$ with corresponding $\beta$ such that

$$
c \leq \sup _{u \in \bar{Q}} I\left(h_{1}(u)\right) \leq c+\varepsilon \text { and } h_{t}(\partial Q) \subset I^{\frac{\alpha-\omega}{2}-\beta} .
$$

Since $h \in \Lambda, h_{t}$ maps bounded sets on bounded sets, due to the definition of $\Gamma$, hence $h_{1}(\bar{Q})$ is bounded. Therefore, there is an $R \in \mathbb{N}$ such that $h_{1}(\bar{Q}) \subset B_{\frac{R}{2}}$. By Lemma 1.3, with $\varrho=\frac{1}{2} \min (\beta, \varepsilon)$, there exist $\eta \in \Gamma$ and $k \in \mathbb{N}$ such that $\eta_{t}^{k s}$ satisfies $\left.i\right)$ and $\left.i i\right)$ of that lemma. Let $g_{t}(u)=\eta_{t}^{k s}\left(h_{t}(u)\right)$, provided that $h_{t}(\partial Q) \subset I^{\frac{\alpha-\omega}{2}-\beta}$, in view of $\left.i\right)$ Lemma 1.3. Hence $g \in \Lambda$ and from 1.2 it follows that

$$
c \leq \sup _{u \in \bar{Q}} I\left(g_{1}(u)\right)
$$

From 1.36), $I\left(h_{1}(u)\right) \leq c+\varepsilon$ for all $u \in \bar{Q}$. Thus, if $h_{1}(u) \in I^{-1}([c-\varepsilon, c+\varepsilon])$, by 1.35 it is possible to apply $i i)$ of Lemma 1.3 to conclude that $g_{1}(u) \in I^{c-\frac{\varepsilon}{2}}$. On the other hand, if $h_{1}(u) \in I^{c-\varepsilon}$, then $i$ ) of Lemma 1.3 yields

$$
I\left(g_{1}(u)\right)=I\left(\eta_{1}^{k s}\left(h_{1}(u)\right)\right) \leq I\left(h_{1}(u)\right)+\varrho \leq c-\varepsilon+\frac{\varepsilon}{2}=c-\frac{\varepsilon}{2},
$$

thus $g_{1}(u) \in I^{c-\frac{\varepsilon}{2}}$ by the choice of $\varrho$. Consequently, it follows that

$$
\sup _{u \in \bar{Q}} I\left(g_{1}(u)\right) \leq c-\frac{\varepsilon}{2},
$$

which contradicts (1.37) and the theorem is proved. 


\section{- \\ Applications on Indefinite Problems}

This chapter aims to present applications for the abstract result developed in Chapter 1. Since Theorem 1.4 works for Cerami sequences, the natural applications are asymptotically linear problems. This chapter contains three sections, in the first one, a periodic solution is obtained for a Hamiltonian System, in the second one, by applying similar arguments, a nonlinear Schrödinger equation corresponding to the Hamiltonian System in the previous section is solved, and in the last one, two application on nonlinear Schrödinger equations with different spectra are studied.

\subsection{An Asymptotically Quadratic Hamiltonian System via an Abstract Linking Theorem}

The goal of this section is to study Hamiltonian systems $(H S)$ as below

$$
\dot{z}=\mathscr{I} H_{z}(t, z), \quad z=(p, q) \in \mathbb{R}^{2 N},
$$

where $\mathscr{I}=\left(\begin{array}{cc}0 & -I \\ I & 0\end{array}\right)$ and $H \in C^{1}\left(\mathbb{R} \times \mathbb{R}^{2 N}, \mathbb{R}\right)$ has the form

$$
H(t, z)=\frac{1}{2} V(t) z \cdot z+R(t, z)
$$

Assume that $V(t)$ satisfies

$\left(V_{0}\right) V(t)$ is continuous, $2 \pi$-periodic, symmetric $2 N \times 2 N$-matrix valued function.

Moreover, on $R(t, z)$ assume the hypotheses

$\left(R_{1}\right) \quad R(t, z) \geq 0$, for all $z \in \mathbb{R}^{2 N}, t \in \mathbb{R}$ and $R_{z}(t, z)=o(|z|)$, uniformly in $t$ as $z \rightarrow 0$; 
$\left(R_{2}\right) \quad R_{z}(t, z)=M(t) z+r_{z}(t, z)$, with $M$ a continuous, $2 \pi$-periodic, symmetric $2 N \times 2 N$ matrix valued function and $r_{z}(t, z)=o(|z|)$, uniformly in $t$ as $|z| \rightarrow+\infty$;

$\left(R_{3}\right)$ Setting $A$ as the operator of $L^{2}\left(S^{1}, \mathbb{R}^{2 N}\right)$ given by $A:=-\left(\mathscr{I} \frac{d}{d t}+V(t)\right)$,

$$
a_{0}:=\inf _{\substack{t \in \mathbb{R}, z \in \mathbb{R}^{2 N} \\|z|=1}}[M(t) z \cdot z]>\lambda^{+}=\inf [\sigma(A) \cap(0,+\infty)]
$$

$\left(R_{4}\right)$ Defining $\mathscr{O}$ as the operator of $L^{2}\left(S^{1}, \mathbb{R}^{2 N}\right)$ given by $\mathscr{O}:=A-\mathscr{M}$, where $\mathscr{M}$ denotes the operator multiplication by $M(t)$,

$$
0 \notin \sigma_{p}(\mathscr{O})
$$

Remark 2.1. Since $R$ is asymptotically quadratic there exists an upper bound for the rate at which $H(t, z) \rightarrow+\infty$ as $|t| \rightarrow+\infty$, that is $|H(t, z)| \leq \frac{1}{2}\left(|| V \|_{\infty}+r_{\infty}\right)|z|^{2}$, where $\|\cdot\|_{\infty}$ denotes the norm in $L^{\infty}\left(\mathbb{R}^{2 N} \times \mathbb{R}^{2 N}\right)$ and $r_{\infty}>0$ is chosen such that $|R(t, z)| \leq r_{\infty}|z|^{2}$ due to $\left(R_{1}\right)-\left(R_{2}\right)$.

\subsubsection{Variational Structure}

Inspired by [7], $(H S)$ is treated in $E$, the space of $2 N$-tuples of $2 \pi$-periodic functions which possess a weak derivative of order $\frac{1}{2}$. Namely, let $L^{2}\left(S^{1}, \mathbb{R}^{m}\right)$ denote the set of $m$-tuples of $2 \pi$-periodic functions which are square integrable. If $z \in L^{2}\left(S^{1}, \mathbb{R}^{m}\right)$ it has a Fourier expansion $z=\sum_{j \in \mathbb{Z}} a_{j} e^{i j t}$, where $a_{j} \in \mathbb{C}^{m}, a_{-j}=\bar{a}_{j}$ and $\sum_{j \in \mathbb{Z}}\left|a_{j}\right|^{2}<+\infty$. Let $W^{\theta, 2}\left(S^{1}, \mathbb{R}^{m}\right)=\left\{z \in L^{2}\left(S^{1}, \mathbb{R}^{m}\right):\|z\|_{W^{\theta, 2}}<+\infty\right\}$ be the Sobolev space with the norm

$$
\|z\|_{W^{\theta, 2}} \equiv\left(\sum_{j \in \mathbb{Z}}\left(1+|j|^{2 \theta}\right)\left|a_{j}\right|^{2}\right)^{\frac{1}{2}} .
$$

Choose $\theta=\frac{1}{2}, m=2 N$ and then $E:=W^{\frac{1}{2}, 2}\left(S^{1}, \mathbb{R}^{2 N}\right)$, with the norm above. It is important to highlight that by [43] (cf. Proposition 6.6., page 36), for any $\beta \in[1,+\infty$ ), $E$ is compactly embedded in $L^{\beta}\left(S^{1}, \mathbb{R}^{2 N}\right)$. In particular, there is an $\alpha_{\beta}>0$ such that $\|z\|\left\|_{\beta} \leq \alpha_{\beta}\right\| z \|$, for all $z \in E$.

Observe that, for smooth $z=(p, q) \in E$, where $p$ and $q$ are each $n$-tuples, setting

$$
\begin{aligned}
Q_{A}(z) & :=\int_{0}^{2 \pi} p(t) \cdot \dot{q}(t) d t-\frac{1}{2} \int_{0}^{2 \pi} V(t) z(t) \cdot z(t) d t \\
& =\int_{0}^{2 \pi}-\frac{1}{2}\left(\mathscr{I} \frac{d}{d t}\right) z(t) \cdot z(t) d t-\frac{1}{2} \int_{0}^{2 \pi} V(t) z(t) \cdot z(t) d t \\
& =\frac{1}{2}(A z, z)_{L^{2}\left(S^{1}, \mathbb{R}^{2 N}\right)},
\end{aligned}
$$


since $V$ is bounded, in view of $\left(V_{0}\right)$ it follows that,

$$
\left|Q_{A}(z)\right| \leq C\left[\left(\sum_{j \in \mathbb{Z}}|j|\left|p_{j}\right|^{2}\right)^{\frac{1}{2}}\left(\sum_{j \in \mathbb{Z}}|j|\left|q_{j}\right|^{2}\right)^{\frac{1}{2}}+\left(\sum_{j \in \mathbb{Z}}\left|p_{j}\right|^{2}\right)^{\frac{1}{2}}\left(\sum_{j \in \mathbb{Z}}\left|q_{j}\right|^{2}\right)^{\frac{1}{2}}\right] \leq\left. C\|z\|\right|_{W^{\frac{1}{2}, 2}} ^{2} .
$$

Therefore, $Q_{A}$ extends to all of $E$ as a continuous quadratic form. If $E^{0}, E^{+}, E^{-}$are the closed subspaces of $E$ on which $Q_{A}$ is null, positive and negative definite, then $E=E^{0} \oplus E^{+} \oplus E^{-}$. Moreover, if

$$
\begin{aligned}
B_{Q_{A}}[z, \xi] & =\int_{0}^{2 \pi}(p(t) \cdot \dot{\psi}(t)+\phi(t) \cdot \dot{q}(t)) d t-\int_{0}^{2 \pi} V(t) z(t) \cdot \xi(t) d t \\
& =\int_{0}^{2 \pi}-\left(\mathscr{I} \frac{d}{d t}+V(t)\right) \cdot \xi(t) d t \\
& =(A z, \xi)_{L^{2}\left(S^{1}, \mathbb{R}^{2 N}\right)},
\end{aligned}
$$

is the bilinear form associated to $Q_{A}$ and $z=(p, q), \xi=(\phi, \psi)$ belong to distinct such subspaces, then $B_{Q_{A}}[z, \xi]=0$ and $Q_{A}(z+\xi)=Q_{A}(z)+Q_{A}(\xi)$. In addition $E^{0}, E^{+}, E^{-}$are mutually orthogonal in $L^{2}\left(S^{1}, \mathbb{R}^{2 N}\right)$. Hence, for $z=z^{0}+z^{+}+z^{-} \in E$, it is suitable to take as an equivalent norm in $E$ the expression

$$
\|z\|^{2}=\|z\|_{E}^{2}:=\left\|z^{0}\right\|_{L^{2}\left(S^{1}, \mathbb{R}^{2 N}\right)}^{2}+Q_{A}\left(z^{+}\right)-Q_{A}\left(z^{-}\right)
$$

and the associated inner product, obtained by means of (2.3), which makes $E$ a Hilbert space with $E^{0}, E^{+}, E^{-}$orthogonal subspaces of $E$.

Remark 2.2. Due to $\left(V_{0}\right)$ the operator multiplication by $V(t)$ is compact, hence the essential spectrum of operator $A$ is the same of $A+V(t)=-\mathscr{I} \frac{d}{d t}, \quad$ namely $\sigma_{\text {ess }}(A)=\sigma_{\text {ess }}(A+$ $V(t))=\{-1,1\}$ (cf. [44], Corollary 2 page 113 and [7], page 262). Then, $\operatorname{ker}(A)=E^{0}$ is finite dimensional, since $0 \notin \sigma_{\text {ess }}(A)$, and either 0 is a isolated point of $\sigma(A)$ or $0 \notin \sigma(A)$, hence $0<\inf [\sigma(A) \cap(0,+\infty)]=\lambda^{+}<a_{0}$, therefore $a_{0}>0$ in $\left(R_{3}\right)$. Furthermore, $\pm 1 \in \sigma_{\text {ess }}(A)$ are isolated points in $\sigma_{\text {ess }}(A)$, thus they are eigenvalues of infinity multiplicity, hence $E_{1}$ and $E_{2}$ are both infinite dimensional. In addition, it means that operator $A$ has at least two eigenvalues, being one positive and the other one negative. Thus, defining $\lambda^{+}, \lambda^{-}$as respectively the smallest positive and largest negative eigenvalues of $A$, they are well defined and $0<\left|\lambda^{-}\right|,\left|\lambda^{+}\right| \leq 1$. Therefore, for $z^{+} \in E^{+}$and $z^{-} \in E^{-}$, Spectral Theory (cf. [9], Theorem 1.1', page 394) asserts that

$$
Q_{A}\left(z^{+}\right) \geq \lambda^{+}\left\|z^{+}\right\|_{L^{2}\left(S^{1}, \mathbb{R}^{2 N}\right)}^{2} \text { and } Q_{A}\left(z^{-}\right) \leq \lambda^{-}\left\|z^{-}\right\|_{L^{2}\left(S^{1}, \mathbb{R}^{2 N}\right)}^{2} .
$$

An example of $V(t)$ satisfying $\left(V_{0}\right)$ is given by $V(t)=\cos (t)$. Moreover, since $\sigma(A)$ is pure point, choosing $a_{0}>1$ a constant such that $a_{0} \notin \sigma(A)$, and setting $M(t)=a_{0}$, it implies that $R(t, z)$ satisfies $\left(R_{4}\right)$. 
Let $I(z)=Q_{A}(z)-\int_{0}^{2 \pi} R(t, z(t)) d t$, for all $z \in E$, be the functional associated to $(H S)$. By $\left(R_{1}\right)-\left(R_{2}\right)$ and provided that $H \in C^{1}\left(\mathbb{R} \times \mathbb{R}^{2 N}, \mathbb{R}\right), I$ is well defined and $I \in C^{1}(E, \mathbb{R})$. Thus, a weak solution to $(H S)$ is a critical point to $I$, a function $z \in E$ such that for all $\phi \in E$

$$
I^{\prime}(z) \phi=Q_{A}^{\prime}(z) \phi-\int_{0}^{2 \pi} R_{z}(t, z(t)) \phi(t) d t=0 .
$$

Via the discussion above about $Q_{A}(z), I$ is indefinite, henceforth the goal is to apply Theorem 1.4 to get a critical point to $I$, namely a periodic solution to $(H S)$. The main result holds.

Theorem 2.1. Suppose $H \in C^{1}\left(\mathbb{R} \times \mathbb{R}^{2 N}, \mathbb{R}\right)$ consider I such that $\left(V_{0}\right)$ and $\left(R_{1}\right)-\left(R_{4}\right)$ hold. Then $(H S)$ possess a $2 \pi$-periodic, nontrivial, weak solution.

First of all, note that $I$ satisfies $\left(I_{1}\right)$ of Theorem 1.4 In fact, setting $E_{1}:=E^{+}$and $E_{2}:=E^{-} \oplus E^{0}$, it yields $E_{2}^{\perp}=E_{1}$. Then, define $L_{i}: E_{i} \rightarrow E_{i}$, for all $z \in E_{i}$, as given by

$$
\left(L_{i} z, \xi\right)_{E}=Q_{A}^{\prime}(z) \xi=B_{Q_{A}}[z, \xi]=(A z, \xi)_{L^{2}\left(S^{1}, \mathbb{R}^{2 N}\right)},
$$

for all $\xi \in E_{i}, i=1,2$, where $Q_{A}^{\prime}(z) \xi$ denotes Fréchet derivative of $Q_{A}$ at $z$ acting on $\xi$. Hence, $L=L_{1}+L_{2}: E_{1} \oplus E_{2} \rightarrow E_{1} \oplus E_{2}$ is a well defined, linear, bounded operator and satisfies

$$
Q_{A}(z)=\frac{1}{2}(A z, z)_{L^{2}\left(S^{1}, \mathbb{R}^{2 N}\right)}=\frac{1}{2} \int_{0}^{2 \pi}-\left(\mathscr{I} \frac{d}{d t}+V(t)\right) z \cdot z=\frac{1}{2} Q_{A}^{\prime}(z) z=\frac{1}{2}(L z, z)_{E} .
$$

Thus, setting $B(z):=-\int_{0}^{2 \pi} R(t, z(t)) d t$, for all $z \in E$, it is possible to write

$$
I(z)=\frac{1}{2}(L z, z)+B(z)
$$

satisfying $\left(I_{1}\right)$.

\subsubsection{Setting Compactness}

In this subsection two lemmas are proved so as to guarantee that $I$ satisfies hypothesis $\left(I_{2}\right)$ in Theorem 1.4 .

Lemma 2.1. If $R \in C\left(\mathbb{R} \times \mathbb{R}^{2 N}, \mathbb{R}\right)$, then $B$ is weakly continuous.

Proof. Let $\left(z_{n}\right) \in E$ be a sequence and suppose $z_{n} \rightarrow z$ in $E$. Provided that $E$ is compactly embedded in $L^{\beta}\left(S^{1}, \mathbb{R}^{2 N}\right)$, for all $\beta \in[1,+\infty)$, it yields $z_{n} \rightarrow z$ in $L^{\beta}\left(S^{1}, \mathbb{R}^{2 N}\right)$ for $\beta=2$ and hence there exists $\zeta \in L^{2}\left(S^{1}, \mathbb{R}^{2 N}\right)$ such that $\left|z_{n}(t)\right| \leq \zeta(t)$ almost everywhere in $S^{1}$, for all $n \in \mathbb{N}$. Since $R$ is continuous and $|R(t, z)| \leq r_{\infty}|z|^{2}$ for all $t \in \mathbb{R}$ and $z \in \mathbb{R}^{2 N}$, then 
$R\left(t, z_{n}(t)\right) \rightarrow R(t, z(t))$, almost everywhere in $S^{1}$ and

$$
\left|R\left(\cdot, z_{n}(\cdot)\right)\right| \leq r_{\infty}\left|z_{n}(\cdot)\right|^{2} \leq r_{\infty} \zeta^{2}(\cdot) \in L^{1}\left(S^{1}, \mathbb{R}^{2 N}\right)
$$

thus by Lebesgue Dominated Convergence Theorem,

$$
\int_{0}^{2 \pi} R\left(t, z_{n}(t)\right) d t \rightarrow \int_{0}^{2 \pi} R(t, z(t)) d t
$$

as $n \rightarrow+\infty$. Therefore $B\left(z_{n}\right) \rightarrow B(z)$ and $B$ is weakly continuous.

Lemma 2.2. Assume that $R \in C^{1}\left(\mathbb{R} \times \mathbb{R}^{2 N}, \mathbb{R}\right)$, then $B$ is uniformly differentiable on bounded sets of $E$.

Proof. Note that $\left|R_{z}(t, z)\right| \leq 2 r_{\infty}|z| \leq 2 r_{\infty}\left(1+|z|^{s}\right)$, for $1 \leq s<+\infty$ and $E$ is a subspace of $L^{1}\left(S^{1}, \mathbb{R}^{2 N}\right)$ with $\|z\|_{r} \leq \alpha_{r}\|z\|$, for all $z \in E$ and for all $r \in[2,+\infty)$, due to the embeddings of $E$ on Lebesgue Spaces. Then Proposition 3.12 in [7] implies that $B(z)$ is uniformly differentiable on bounded subsets of $E$.

\subsubsection{Linking Geometry}

Under the purpose of establishing a linking geometry, set $S:=\partial B_{\rho} \cap E_{1}$ and

$$
Q:=\left\{r e+z_{2}: r \geq 0, z_{2} \in E_{2},\left\|r e+z_{2}\right\| \leq r_{1}\right\}
$$

where $0<\rho<r_{1}$ are constants and $e \in E_{1},\|e\|=1$, is chosen suitably. Indeed, due to $\left(R_{3}\right)$ and 2.4 , let $e \in E_{1}$ be a unitary eigenvector associated to $\lambda^{+}$, the first positive eigenvalue, then it satisfies

$$
\begin{aligned}
1=\|e\|^{2}=Q_{A}(e) & =\frac{1}{2}(A e, e)_{L^{2}\left(S^{1}, \mathbb{R}^{2 N}\right)} \\
& =\frac{1}{2} \lambda^{+}\|e\|_{L^{2}\left(S^{1}, \mathbb{R}^{2 N}\right)}^{2} \\
& <\frac{1}{2} a_{0}\|e\|_{L^{2}\left(S^{1}, \mathbb{R}^{2 N}\right)}^{2} \\
& \leq \frac{1}{2} \int_{0}^{2 \pi} M(t) e(t) \cdot e(t) d t .
\end{aligned}
$$

Choosing such $e$, it is possible by means of 2.5 to show that for sufficiently large $r_{1}>0$, $\left.I\right|_{S} \geq \alpha>0$ and $\left.I\right|_{\partial Q} \leq 0$ hold, for some $\alpha>0$. Moreover, as noticed before, $S$ and $Q$ "link". Hence, $I$ satisfies $\left(I_{3}\right)$ for some $\alpha>0, \omega=0$ and arbitrary $v \in E_{2}$.

Lemma 2.3. Assume that $H \in C^{1}\left(\mathbb{R} \times \mathbb{R}^{2 N}, \mathbb{R}\right)$. Under hypotheses $\left(V_{0}\right)$ on $V$ and $\left(R_{1}\right)-\left(R_{3}\right)$ on $R, I$ satisfies $\left(I_{3}\right)$. 
Proof. Note that from $\left(R_{1}\right)-\left(R_{2}\right)$, given $\varepsilon>0$ and $2<p<2^{*}$, there exists $C_{\varepsilon}>0$ such that

$$
|R(t, z)| \leq \frac{\varepsilon}{2}|z|^{2}+\frac{C_{\varepsilon}}{p}|z|^{p} \text { and }\left|R_{z}(t, z)\right| \leq \varepsilon|z|+C_{\varepsilon}|z|^{p-1}
$$

for all $z \in \mathbb{R}^{2 N}$, and for all $t \in \mathbb{R}$. Since $S \subset E_{1}$, for all $z_{1} \in S$, it yields

$$
\begin{aligned}
I\left(z_{1}\right) & =\frac{1}{2}\left\|z_{1}\right\|^{2}-\int_{0}^{2 \pi} R\left(t, z_{1}(t)\right) d t \\
& \geq \frac{1}{2} \rho^{2}-\int_{0}^{2 \pi}\left(\frac{\varepsilon}{2}\left|z_{1}(t)\right|^{2}+\frac{C_{\varepsilon}}{p}\left|z_{1}(t)\right|^{p}\right) d t \\
& \geq \frac{1}{2} \rho^{2}-\left(\frac{\varepsilon}{2} C_{2}^{2}\left\|z_{1}\right\|^{2}+\frac{C_{\varepsilon}}{p} C_{p}^{p}\left\|z_{1}\right\|^{p}\right) \\
& =\rho^{2}\left[\frac{1}{2}\left(1-\varepsilon C_{2}^{2}\right)-\frac{C_{\varepsilon}}{p} C_{p}^{p} \rho^{p-2}\right] \\
& \geq \rho^{2}\left(d_{1}-d_{2}\right)=\alpha>0,
\end{aligned}
$$

where $\varepsilon, \rho$ are sufficiently small, such that $1>\varepsilon C_{2}^{2}$ and also

$$
d_{1}:=\frac{1}{2}\left(1-\varepsilon C_{2}^{2}\right)>\frac{C_{\varepsilon}}{p} C_{p}^{p} \rho^{p-2}=: d_{2} .
$$

Therefore, from (2.6), $\left(I_{3}\right)(i)$ holds for $I$.

In order to prove that $I$ satisfies $\left(I_{3}\right)$ (ii) in Theorem 1.4, with $\omega=0$, observe that $I(z) \leq 0$, for all $z \in E_{2}=E^{-} \oplus E^{0}$, then it suffices to show that $I(r e+z) \leq 0$ for $r>0, z \in E_{2}$ and $\|r e+z\| \geq r_{1}$, for some $r_{1}>0$ large enough. Arguing indirectly assume that for some sequence $\left(r_{n} e+z_{n}\right) \subset \mathbb{R}^{+} e \oplus E_{2}$ with $\left\|r_{n} e+z_{n}\right\| \rightarrow+\infty, I\left(r_{n} e+z_{n}\right)>0$ holds, for all $n \in \mathbb{N}$. Seeking a contradiction, set

$$
\tilde{z}_{n}:=\frac{r_{n} e+z_{n}}{\left\|r_{n} e+z_{n}\right\|}=s_{n} e+w_{n}
$$

where $s_{n} \in \mathbb{R}^{+}, w_{n}=w_{n}^{-}+w_{n}^{0} \in E_{2}=E^{-} \oplus E^{0}$ and $\left\|\tilde{z}_{n}\right\|=1$. Provided that $\left(\tilde{z}_{n}\right)$ is bounded, up to subsequences it follows that $\tilde{z}_{n} \rightarrow \tilde{z}=s e+w$ in $E$. Then, up to subsequences, $\tilde{z}_{n}(t) \rightarrow \tilde{z}(t)$ almost everywhere in $\mathbb{R}, s_{n} \rightarrow s$ in $\mathbb{R}^{+}, w_{n}^{-} \rightarrow w$ in $E$, and $w_{n}^{0} \rightarrow w^{0}$ in $E$, since $\left(w_{n}^{0}\right) \subset E^{0}$ and $E^{0}$ is finite dimensional. Seeing that

$$
1=\left\|s_{n} e+w_{n}\right\|^{2}=s_{n}^{2}+\left\|w_{n}^{-}\right\|^{2}+\left\|w_{n}^{0}\right\|^{2}
$$

it follows that $0 \leq s_{n}^{2} \leq 1$, and it yields

$$
\begin{aligned}
\frac{I\left(r_{n} e+z_{n}\right)}{\left\|r_{n} e+z_{n}\right\|^{2}} & =s_{n}^{2}\|e\|^{2}-\left\|w_{n}^{-}\right\|^{2}-\int_{0}^{2 \pi} \frac{R\left(t, r_{n} e(t)+z_{n}(t)\right)}{\left\|r_{n} e+z_{n}\right\|^{2}} d t \\
& =2 s_{n}^{2}-1-\left\|w_{n}^{0}\right\|^{2}-\int_{0}^{2 \pi} \frac{R\left(t, r_{n} e(t)+z_{n}(t)\right)}{\left\|r_{n} e+z_{n}\right\|^{2}} d t>0
\end{aligned}
$$


hence $\frac{1}{2} \leq s^{2} \leq 1$. Moreover, from 2.5 it follows that

$$
1<\frac{1}{2} \int_{0}^{2 \pi} M(t) e(t) \cdot e(t) d t
$$

Hence,

$$
\begin{aligned}
0 & >s^{2}-s^{2} \frac{1}{2} \int_{0}^{2 \pi} M(t) e(t) \cdot e(t) d t \\
& \geq s^{2}\left(1-\frac{1}{2} \int_{0}^{2 \pi} M(t) e(t) \cdot e(t) d t\right)-\left(1+\left\|w^{0}\right\|^{2}-s^{2}\right)-\frac{1}{2} \int_{0}^{2 \pi} M(t) w(t) \cdot w(t) d t \\
& =s^{2}\left(2-\frac{1}{2} \int_{0}^{2 \pi} M(t) e(t) \cdot e(t) d t\right)-1-\left\|w^{0}\right\|^{2}-\frac{1}{2} \int_{0}^{2 \pi} M(t) w(t) \cdot w(t) d t .
\end{aligned}
$$

On the other hand, from Remark 2.1 and since $\tilde{z}_{n}$ is convergent in $L^{2}\left(S^{1}, \mathbb{R}^{2 N}\right)$, there exists some $\psi \in L^{1}\left(S^{1}, \mathbb{R}\right)$ such that for sufficiently large $n \in \mathbb{N},\left\|r_{n} e+z_{n}\right\|^{2}>1$ and then

$$
\left|\frac{R\left(\cdot, r_{n} e(\cdot)+z_{n}(\cdot)\right)}{\left\|r_{n} e+z_{n}\right\|^{2}}\right| \leq r_{\infty}\left|\tilde{z}_{n}(\cdot)\right|^{2} \leq \psi(\cdot) \in L^{1}\left(S^{1}, \mathbb{R}\right)
$$

Moreover, provided that $\left\|r_{n} e+z_{n}\right\| \rightarrow+\infty$, and $\tilde{z}_{n}(t) \rightarrow \tilde{z}(t) \neq 0$, almost everywhere in $\operatorname{supp}(\tilde{z})$ as $n \rightarrow+\infty$, from $\left(R_{2}\right)$ it yields

$$
\frac{R\left(t, r_{n} e(t)+z_{n}(t)\right)}{\left\|r_{n} e+z_{n}\right\|^{2}}=\frac{1}{2} M(t) \tilde{z}_{n}(t) \cdot \tilde{z}_{n}(t)+\frac{r\left(t, \tilde{z}_{n}(t)|| r_{n} e+z_{n} \|\right)\left|\tilde{z}_{n}(t)\right|^{2}}{\left|\tilde{z}_{n}(t)\right|^{2}|| r_{n} e+z_{n} \|^{2}} \rightarrow \frac{1}{2} M(t) \tilde{z}(t) \cdot \tilde{z}(t),
$$

almost everywhere in $\operatorname{supp}(\tilde{z}) \cap S^{1}$ as $n \rightarrow+\infty$. Note that, $\operatorname{supp}(\tilde{z}) \neq \emptyset$, because $\tilde{z}=s e+w$, with $\operatorname{supp}(e) \neq \emptyset$ and $(e, w)_{L^{2}\left(S^{1}, \mathbb{R}^{2 N}\right)}=0$. Thus, by Lebesgue Dominated Convergence Theorem,

$$
\int_{0}^{2 \pi} \frac{R\left(t, r_{n} e(t)+z_{n}(t)\right)}{\left\|r_{n} e+z_{n}\right\|^{2}} d t \rightarrow \frac{1}{2} \int_{0}^{2 \pi} M(t)(s e(t)+w(t)) \cdot(s e(t)+w(t)) d t
$$

as $n \rightarrow+\infty$. Passing to the limit in (2.7) as $n \rightarrow+\infty$, it yields

$$
\begin{aligned}
0 & \leq 2 s^{2}-1-\left\|w^{0}\right\|^{2}-\frac{1}{2} \int_{0}^{2 \pi} M(t)\left(s^{2} e(t) \cdot e(t)+w(t) \cdot w(t)\right) d t \\
& =s^{2}\left(2-\frac{1}{2} \int_{0}^{2 \pi} M(t) e(t) \cdot e(t) d t\right)-1-\left\|w^{0}\right\|^{2} \\
& -\frac{1}{2} \int_{0}^{2 \pi} M(t) w(t) \cdot w(t) d t,
\end{aligned}
$$

which is contrary to 2.8. Therefore the result holds. 


\subsubsection{Boundedness of Cerami Sequences}

This section is dedicated to show the boundedness of Cerami sequences required in $\left(I_{4}\right)$ and finally to prove Theorem 2.1 .

Lemma 2.4. Suppose that $H \in C^{1}\left(\mathbb{R} \times \mathbb{R}^{2 N}, \mathbb{R}\right), V$ satisfies $\left(V_{0}\right)$ and $R$ satisfies $\left(R_{1}\right)-\left(R_{4}\right)$, then I satisfies $\left(I_{4}\right)$.

Proof. Let $b>0$ be an arbitrary constant, and take $\left(z_{n}\right) \subset I^{-1}([c-b, c+b])$ such that $\left(1+\left\|z_{n}\right\|\right)\left\|I^{\prime}\left(z_{n}\right)\right\| \rightarrow 0$. Then $\left(z_{n}\right)$ is bounded. In fact, suppose by contradiction that $\left\|z_{n}\right\| \rightarrow+\infty$, up to subsequences. Setting $\tilde{z}_{n}:=\frac{z_{n}}{\left\|z_{n}\right\|}$, it is bounded, hence $\tilde{z}_{n} \rightarrow \tilde{z}$ in $E$ and $\tilde{z}_{n} \rightarrow \tilde{z}$ in $L^{s}\left(S^{1}, \mathbb{R}^{2 N}\right)$, for $s \in[1,+\infty)$, due to the compact embeddings previously mentioned. Writing $z_{n}=z_{n}^{+}+z_{n}^{-}+z_{n}^{0} \in E^{+} \oplus E^{-} \oplus E^{0}$, by the choice of $z_{n}$ it satisfies

$$
\begin{aligned}
o_{n}(1) & =I^{\prime}\left(z_{n}\right) \frac{z_{n}^{+}}{\left\|z_{n}\right\|^{2}} \\
& =\frac{1}{\left\|z_{n}\right\|} I^{\prime}\left(z_{n}\right) \tilde{z}_{n}^{+} \\
& =\left\|\tilde{z}_{n}^{+}\right\|^{2}-\int_{0}^{2 \pi} \frac{R_{z}\left(t, z_{n}(t)\right) \tilde{z}_{n}^{+}(t)}{\left|z_{n}(t)\right|}\left|\tilde{z}_{n}(t)\right| d t,
\end{aligned}
$$

and

$$
\begin{aligned}
o_{n}(1) & =I^{\prime}\left(z_{n}\right) \frac{z_{n}^{-}}{\left\|z_{n}\right\|^{2}} \\
& =\frac{1}{\left\|z_{n}\right\|} I^{\prime}\left(z_{n}\right) \tilde{z}_{n}^{-} \\
& =-\left\|\tilde{z}_{n}^{-}\right\|^{2}-\int_{0}^{2 \pi} \frac{R_{z}\left(t, z_{n}(t)\right) \tilde{z}_{n}^{-}(t)}{\left|z_{n}(t)\right|}\left|\tilde{z}_{n}(t)\right| d t .
\end{aligned}
$$

Subtracting 2.12) from 2.11), and using that $1=\left\|\tilde{z}_{n}^{+}\right\|^{2}+\left\|\tilde{z}_{n}^{-}\right\|^{2}+\left\|\tilde{z}_{n}^{0}\right\|^{2}$, it yields

$$
o_{n}(1)=1-\left\|\tilde{z}_{n}^{0}\right\|^{2}-\int_{0}^{2 \pi} \frac{R_{z}\left(t, z_{n}(t)\right)\left[\tilde{z}_{n}^{+}(t)-\tilde{z}_{n}^{-}(t)\right]}{\left|z_{n}(t)\right|}\left|\tilde{z}_{n}(t)\right| d t .
$$

Provided that $\left(\tilde{z}_{n}^{0}\right) \subset E^{0}$, which is finite dimensional, then weak convergence implies that $\tilde{z}_{n}^{0} \rightarrow \tilde{z}^{0}$ in $E$. Furthermore, since $\tilde{z}_{n} \rightarrow \tilde{z}$ in $L^{2}\left(S^{1}, \mathbb{R}^{2 N}\right)$, there exist $\psi^{+}, \psi^{-} \in L^{2}\left(S^{1}, \mathbb{R}\right)$ such that $\left|\tilde{z}_{n}^{+}(t)\right| \leq \psi^{+}(t)$ and $\left|\tilde{z}_{n}^{-}(t)\right| \leq \psi^{-}(t)$, almost everywhere in $\mathbb{R}$, hence from Remark 2.1 it follows that

$$
\left|\frac{R_{z}\left(t, z_{n}(t)\right)\left[\tilde{z}_{n}^{+}(t)-\tilde{z}_{n}^{-}(t)\right]}{\left|z_{n}(t)\right|}\right| \tilde{z}_{n}(t)|| \leq 2 r_{\infty}\left[\left(\psi^{+}(\cdot)\right)^{2}+\left(\psi^{-}(\cdot)\right)^{2}\right] \in L^{1}\left(S^{1}, \mathbb{R}\right) .
$$

In addition, since $\tilde{z}_{n} \rightarrow \tilde{z}$ in $L^{2}\left(S^{1}, \mathbb{R}^{2 N}\right),\left|z_{n}(t)\right| \rightarrow+\infty$, for all $t$ such that $\tilde{z}(t) \neq 0$, from $\left(R_{2}\right)$ 
it follows that

$$
\begin{aligned}
\frac{R_{z}\left(t, z_{n}(t)\right)\left[\tilde{z}_{n}^{+}(t)-\tilde{z}_{n}^{-}(t)\right]}{\left|z_{n}(t)\right|}\left|\tilde{z}_{n}(t)\right| & =\left(M(t) \tilde{z}_{n}(t)+\frac{\left|\tilde{z}_{n}(t)\right| r_{z}\left(t, z_{n}(t)\right)}{\left|z_{n}(t)\right|}\right)\left[\tilde{z}_{n}^{+}(t)-\tilde{z}_{n}^{-}(t)\right] \\
& \rightarrow M(t) \tilde{z}(t) \cdot\left[\tilde{z}^{+}(t)-\tilde{z}^{-}(t)\right]
\end{aligned}
$$

as $n \rightarrow+\infty$. Therefore, by Lebesgue Dominated Convergence Theorem, passing to the limit in (2.13) as $n \rightarrow+\infty$, it yields

$$
\int_{0}^{2 \pi} M(t) \tilde{z}(t) \cdot\left[\tilde{z}^{+}(t)-\tilde{z}^{-}(t)\right] d t=1-\left\|\tilde{z}^{0}\right\|^{2}
$$

On the other hand, given $\phi \in E$, from $\left(R_{2}\right)$ it follows that

$$
\int_{0}^{2 \pi} \frac{R_{z}\left(t, z_{n}(t)\right) \phi(t)}{\left\|z_{n}\right\|} d t=\int_{0}^{2 \pi} M(t) \tilde{z}_{n}(t) \cdot \phi(t) d t+\int_{0}^{2 \pi} \frac{r_{z}\left(t, z_{n}(t)\right) \phi(t)}{\left\|z_{n}\right\|} d t .
$$

Since $\tilde{z}_{n} \rightarrow \tilde{z}$ in $L^{2}\left(S^{1}, \mathbb{R}^{2 N}\right),\left|z_{n}(t)\right| \rightarrow+\infty$ for all $t$ such that $\tilde{z}(t) \neq 0$, then again from $\left(R_{2}\right)$ it follows that

$$
\int_{0}^{2 \pi} \frac{R_{z}\left(t, z_{n}(t)\right) \phi(t)}{\left\|z_{n}\right\|} d t=\int_{0}^{2 \pi} M(t) \tilde{z}_{n}(t) \cdot \phi(t) d t+o_{n}(1),
$$

as $n \rightarrow+\infty$. Hence, using the same arguments as above and applying Lebesgue Dominated Convergence Theorem, it yields

$$
\begin{aligned}
o_{n}(1) & =\frac{I^{\prime}\left(z_{n}\right) \phi}{\left\|z_{n}\right\|} \\
& =\frac{Q_{A}^{\prime}\left(z_{n}\right) \phi}{\left\|z_{n}\right\|}-\int_{0}^{2 \pi} \frac{R_{z}\left(t, z_{n}(t)\right) \phi(t)}{\left\|z_{n}\right\|} d t \\
& =\left(A \tilde{z}_{n}, \phi\right)_{L^{2}\left(S^{1}, \mathbb{R}^{2 N}\right)}-\int_{0}^{2 \pi} M(t) \tilde{z}_{n}(t) \cdot \phi(t) d t+o_{n}(1) \\
& =\left(\mathscr{O} \tilde{z}_{n}, \phi\right)_{L^{2}\left(S^{1}, \mathbb{R}^{2 N}\right)}+o_{n}(1) \\
& =(\mathscr{O} \tilde{z}, \phi)_{L^{2}\left(S^{1}, \mathbb{R}^{2 N}\right)}+o_{n}(1) .
\end{aligned}
$$

Due to 2.15, if $\tilde{z} \neq 0$, it is an eigenvector of $\mathscr{O}$ with eigenvalue 0 . Nevertheless, from $\left(R_{4}\right)$, $0 \notin \sigma_{p}(\mathscr{O})$ thus, $\tilde{z}=0$. It means that $\tilde{z}^{+}=\tilde{z}^{-}=\tilde{z}^{0}=0$ thus, 2.14 yields another contradiction. Therefore, the result holds.

Now, the main result of this section can be proved.

Proof of Theorem 2.1. Provided that $I$ satisfies all assumptions $\left(I_{1}\right)-\left(I_{4}\right)$ in Theorem 1.4, applying it provides a critical point $u \in E$ of $I$, and therefore $u$ is a $2 \pi$-periodic, nontrivial, weak solution to $(H S)$, since $I(u)=c>0$, hence $u \not \equiv 0$. 
Remark 2.3. With minor changes in the arguments above, it is possible to find a periodic solution to a type of problem involving a nonlinear Schrödinger equation corresponding to $(H S)$ :

$$
-\Delta u+V(x) u=g(x, u), \quad x \in \mathbb{R}^{N}, N \geq 3
$$

where, $V(x)$ is $(2 \pi)$-periodic in $x_{i}, i=1, \ldots, N$, and $g(x, s)$ satisfies similar hypotheses as those satisfied by $R_{z}(t, z)$. This problem is going to be studied next section.

\subsection{A Nonlinear Schrödinger Equation Corresponding to a Hamiltonian System}

The goal of this section is to study a type of problem involving a Schrödinger operator and a nonlinearity satisfying hypotheses very similar as those satisfied by $R_{z}(t, z)$ in the Hamiltonian systems $(H S)$ in last section. This type of problem is known as a nonlinear Schrödinger equation corresponding to a Hamiltonian system like $(H S)$, since it is possible to employ analogous arguments to obtain existence of solution to this problem.

More precisely, consider again problem $(P)$ treated in Section 3.3:

$$
-\Delta u+V(x) u=g(x, u), \quad x \in \mathbb{R}^{N}, N \geq 3 .
$$

For this setting $V(x)$ satisfies

$\left(V_{1}^{\prime}\right) \quad V \in C\left(\mathbb{R}^{N}, \mathbb{R}\right)$ is $(2 \pi)$-periodic in $x_{i}, i=1, \ldots, N$;

$\left(V_{2}^{\prime}\right)$ Setting $A:=-\Delta+V(x)$, as an operator of $L^{2}\left(\left(S^{1}\right)^{N}, \mathbb{R}\right)$,

$$
\inf \sigma(A)<0 \text {. }
$$

Moreover, $g(x, s)$ satisfies

$\left(G_{1}\right) g(x, s) \in C\left(\mathbb{R}^{N} \times \mathbb{R}, \mathbb{R}\right), \quad g(x, s)=o(|s|)$ as $|s| \rightarrow 0$, uniformly in $x$ and for all $t \in \mathbb{R}$

$$
G(x, t)=\int_{0}^{t} g(x, s) d s \geq 0
$$

$\left(G_{2}\right) g(x, s)=h(x) s+\tilde{g}(x, s)$, where $h(x)$ and $\tilde{g}(x, s)$ are $(2 \pi)$-periodic in $x_{i}, i=1, \ldots, N$, and $\tilde{g}(x, s)=o(|s|)$ as $|s| \rightarrow+\infty$, uniformly in $x$;

$\left(G_{3}\right) \quad a_{0}=\inf _{x \in \mathbb{R}^{N}} h(x)>\lambda^{+}=\inf [\sigma(A) \cap(0,+\infty)] ;$ 
$\left(G_{4}\right)$ Setting $\mathscr{O}:=A-\mathscr{H}$, where $\mathscr{H}$ is the operator multiplication by $h(x)$ in $L^{2}\left(\left(S^{1}\right)^{N}, \mathbb{R}\right)$,

$$
0 \notin \sigma_{p}(\mathscr{O})
$$

Remark 2.4. From $\left(G_{1}\right)-\left(G_{2}\right)$ there exist $C_{0}>0$ such that $|\tilde{g}(x, s)| \leq C_{0}|s|$ for all $s \in \mathbb{R}$ and $x \in \mathbb{R}^{N}$. Hence, there exist $C>0$ such that

$$
|g(x, s)| \leq h_{\infty}|s|+C_{0}|s| \leq C|s|
$$

for all $s \in \mathbb{R}$ and $x \in \mathbb{R}^{N}$, where $h_{\infty}=\|h\|_{L^{\infty}\left(\left(S^{1}\right)^{N}, \mathbb{R}\right)}$. In addition, it follows that

$$
|G(x, s)| \leq \frac{C}{2}|s|^{2}
$$

for all $s \in \mathbb{R}$ and $x \in \mathbb{R}^{N}$.

\subsubsection{Variational Structure}

Making a parallel with Section 3.3, this problem is treated in a space of ( $2 \pi)$-periodic functions in $x_{i}, i=1, \ldots, N$. Being $A:=-\Delta+V(x)$, an operator of $L^{2}\left(\left(S^{1}\right)^{N}, \mathbb{R}\right)$, and observing that the functional associated to problem $(2.16)$ is given by

$$
I(u)=\frac{1}{2}(A u, u)_{L^{2}\left(\left(S^{1}\right)^{N}, \mathbb{R}\right)}-\int_{\left(S^{1}\right)^{N}} G(x, u) d x
$$

it is natural to define $E:=H^{1}\left(\left(S^{1}\right)^{N}, \mathbb{R}\right)=W^{1,2}\left(\left(S^{1}\right)^{N}, \mathbb{R}\right)$ and to remind that by 43] (cf. Proposition 6.6., page 36) $E$ is compactly embedded in $L^{\beta}\left(\left(S^{1}\right)^{N}, \mathbb{R}\right)$, for any $\beta \in[0,+\infty)$.

Defining $Q_{A}: E \rightarrow \mathbb{R}$ by

$$
Q_{A}(u):=\int_{\left(S^{1}\right)^{N}}|\nabla u(x)|^{2} d x+\int_{\left(S^{1}\right)^{N}} V(x) u^{2}(x) d x=\frac{1}{2}(A u, u)_{L^{2}\left(\left(S^{1}\right)^{N}, \mathbb{R}\right)},
$$

it is a continuous quadratic form on $E$. If $E^{0}, E^{+}, E^{-}$are the closed subspaces of $E$ on which $Q_{A}$ is null, positive and negative definite, then $E=E^{0} \oplus E^{+} \oplus E^{-}$. Moreover, if $B_{Q_{A}}[u, v]=(A u, v)_{L^{2}\left(\left(S^{1}\right)^{N}, \mathbb{R}\right)}$ for all $u, v \in E$, is the bilinear form associated to $Q_{A}$ and $u, v$ belong to distinct such subspaces, then $B_{Q_{A}}[u, v]=0$ and $Q_{A}(u+v)=Q_{A}(u)+Q_{A}(v)$. In addition $E^{0}, E^{+}, E^{-}$are mutually orthogonal in $L^{2}\left(\left(S^{1}\right)^{N}, \mathbb{R}\right)$. Hence, for $u=u^{0}+u^{+}+u^{-} \in E$, it is suitable to take as an equivalent norm in $E$ the expression

$$
\|u\|^{2}=\|u\|_{E}^{2}:=\left\|u^{0}\right\|_{L^{2}\left(\left(S^{1}\right)^{N}, \mathbb{R}\right)}^{2}+Q_{A}\left(u^{+}\right)-Q_{A}\left(u^{-}\right),
$$

and the associated inner product, obtained by means of $B_{Q_{A}}[u, v]$, which makes $E$ a Hilbert space with $E^{0}, E^{+}, E^{-}$orthogonal subspaces of $E$. 
Due to $\left(V_{1}^{\prime}\right)$ the operator multiplication by $V(x)$ is compact from $E \subset L^{2}\left(\left(S^{1}\right)^{N}, \mathbb{R}^{2 N}\right)$ to $L^{2}\left(\left(S^{1}\right)^{N}, \mathbb{R}^{2 N}\right)$, hence the essential spectrum of operator $A$ is the same of $A-V(x)=-\Delta$, namely $\sigma_{\text {ess }}(A)=\sigma_{\text {ess }}(A-V(x))=\emptyset$ (cf. [44, Corollary 2 page 113). Then, $A$ has discrete spectrum and $\operatorname{ker}(A)=E^{0}$ is finite dimensional, since $0 \notin \sigma_{\text {ess }}(A)$ and either 0 is a isolated point of $\sigma(A)$ or $0 \notin \sigma(A)$, hence $0<\inf [\sigma(A) \cap(0,+\infty)]=\lambda^{+}<a_{0}$, therefore $a_{0}>0$ in $\left(G_{3}\right)$. Furthermore, due to $\left(V_{2}\right)$ both $E_{1}$ and $E_{2}$ are nonzero dimensional. In addition, it means that operator $A$ has at least two eigenvalues, being one positive and the other one negative. Thus, defining $\lambda^{+}, \lambda^{-}$as respectively the smallest positive and largest negative eigenvalues of $A$, they are well defined and $0<\left|\lambda^{-}\right|,\left|\lambda^{+}\right| \leq 1$. Therefore, for $u^{+} \in E^{+}$and $u^{-} \in E^{-}$, Spectral Theory in [9] (cf. page 394 Theorem 1.1') asserts that

$$
Q_{A}\left(u^{+}\right) \geq \lambda^{+}\left\|u^{+}\right\|_{L^{2}\left(\left(S^{1}\right)^{N}, \mathbb{R}\right)}^{2} \text { and } Q_{A}\left(u^{-}\right) \leq \lambda^{-}\left\|u^{-}\right\|_{L^{2}\left(\left(S^{1}\right)^{N}, \mathbb{R}\right)}^{2} .
$$

Since $I(u)=Q_{A}(u)-\int_{\left(S^{1}\right)^{N}} G(x, u(x)) d x$, for all $x \in E$, by $\left(V_{1}^{\prime}\right)$ and $\left(G_{1}\right)-\left(G_{2}\right), I$ is well defined and $I \in C^{1}(E, \mathbb{R})$. Thus, a weak solution to $(P)$ is a critical point to $I$, a function $u \in E$ such that for all $v \in E$

$$
I^{\prime}(u) \phi=Q_{A}^{\prime}(u) v-\int_{\left(S^{1}\right)^{N}} g(x, u(x)) v(x) d x=0 .
$$

Via the study above about $Q_{A}(u), I$ is indefinite, henceforth the goal is to apply Theorem 1.4 in order to get a critical point to $I$, namely a periodic solution to $(P)$. The main result holds.

Theorem 2.2. Suppose $\left(V_{1}^{\prime}\right)-\left(V_{2}^{\prime}\right)$ and $\left(G_{1}\right)-\left(G_{4}\right)$ hold. Then problem $(P)$ in (2.16) possess a $(2 \pi)$-periodic in $x_{i}, i=1, \ldots, N$, nontrivial, weak solution.

It is clear that $I$ satisfies $\left(I_{1}\right)$ of Theorem 1.4 . In fact, setting $E_{1}:=E^{+}$and $E_{2}:=E^{-} \oplus E^{0}$, it yields $E_{2}^{\perp}=E_{1}$. Then, define $L_{i}: E_{i} \rightarrow E_{i}$, for all $u \in E_{i}$, as given by

$$
\left(L_{i} u, v\right)_{E}=Q_{A}^{\prime}(u) v=B_{Q_{A}}[u, v]=(A u, v)_{L^{2}\left(\left(S^{1}\right)^{N}, \mathbb{R}\right)}
$$

for all $v \in E_{i}, i=1,2$, where $Q_{A}^{\prime}(u) v$ denotes Fréchet derivative of $Q_{A}$ at $u$ acting on $v$. Hence, $L=L_{1}+L_{2}: E_{1} \oplus E_{2} \rightarrow E_{1} \oplus E_{2}$ is a well defined, linear, bounded operator and satisfies

$$
Q_{A}(u)=\frac{1}{2}(A u, u)_{L^{2}\left(\left(S^{1}\right)^{N}, \mathbb{R}\right)}=\frac{1}{2} Q_{A}^{\prime}(u) u=\frac{1}{2} B_{Q_{A}}[u, u]=\frac{1}{2}(L u, u)_{E} .
$$

Thus, setting $B(u):=-\int_{\left(S^{1}\right)^{N}} G(x, u(x)) d x$, for all $u \in E$, it is possible to write

$$
I(u)=\frac{1}{2}(L u, u)+B(u)
$$

satisfying $\left(I_{1}\right)$. 


\subsubsection{Setting Compactness}

In this subsection assumption $\left(I_{2}\right)$ is verified by means of the following two lemmas.

Lemma 2.5. If $g$ satisfies $\left(G_{1}\right)-\left(G_{2}\right)$, then $B$ is weakly continuous.

Proof. Let $\left(u_{n}\right) \in E$ be a sequence and suppose $u_{n} \rightarrow u$ in $E$. Provided that $E$ is compactly embedded in $L^{\beta}\left(\left(S^{1}\right)^{N}, \mathbb{R}\right)$, for all $\beta \in[1,+\infty)$, it yields $u_{n} \rightarrow u$ in $L^{\beta}\left(\left(S^{1}\right)^{N}, \mathbb{R}\right)$ for $\beta=2$ and hence there exists $\zeta \in L^{2}\left(\left(S^{1}\right)^{N}, \mathbb{R}\right)$ such that $\left|u_{n}(x)\right| \leq \zeta(x)$ almost everywhere in $\left(S^{1}\right)^{N}$, for all $n \in \mathbb{N}$. By $\left(G_{1}\right), G$ is continuous, hence $G\left(x, u_{n}(x)\right) \rightarrow G(x, u(x))$, almost everywhere in $\left(S^{1}\right)^{N}$ and due to Remark 2.4 it follows that

$$
\left|G\left(\cdot, u_{n}(\cdot)\right)\right| \leq \frac{C}{2}\left|u_{n}(\cdot)\right|^{2} \leq \frac{C}{2} \zeta^{2}(\cdot) \in L^{1}\left(\left(S^{1}\right)^{N}, \mathbb{R}\right),
$$

thus by Lebesgue Dominated Convergence Theorem,

$$
\int_{\left(S^{1}\right)^{N}} G\left(x, u_{n}(x)\right) d x \rightarrow \int_{\left(S^{1}\right)^{N}} G(x, u(x)) d x,
$$

as $n \rightarrow+\infty$. Therefore $B\left(u_{n}\right) \rightarrow B(u)$ and $B$ is weakly continuous.

Lemma 2.6. Assume that $g$ satisfies $\left(G_{1}\right)-\left(G_{2}\right)$, then $B$ is uniformly differentiable on bounded sets of $E$.

Proof. First, note that fixed $R>0$ and given $u+v, v \in B_{R}$, it follows that

$$
\begin{aligned}
\left|B(u+v)-B(u)-B^{\prime}(u) v\right| & =\int_{\left(S^{1}\right)^{N}}|G(x, u(x)+v(x))-G(x, u(x))-g(x, u(x)) v(x)| d x \\
& \leq \int_{\left(S^{1}\right)^{N}}|g(x, z(x))-g(x, u(x))||v(x)| d x \\
& \leq C_{2}\|\xi\|_{L^{2}\left(\left(S^{1}\right)^{N}, \mathbb{R}\right)}\|v\|
\end{aligned}
$$

where $\xi(x):=|g(x, z(x))-g(x, u(x))|$ and $z(x)=u(x)+\theta(x) v(x)$, with $0 \leq \theta(x) \leq 1$ given by Mean Value Theorem and $C_{2}>0$ is the constant given by the continuous embedding $E \hookrightarrow$ $L^{2}\left(\left(S^{1}\right)^{N}, \mathbb{R}\right)$.

In order to prove that $B$ is uniformly differentiable on bounded sets of $E$, given $\varepsilon>0$ is sufficient to show there exist $\delta>0$ such that $C_{2}\|\xi\|_{L^{2}\left(\left(S^{1}\right)^{N}, \mathbb{R}\right)} \leq \varepsilon$ for all $u+v, v \in B_{R}$ with $\|v\| \leq \delta$. Seeking a contradiction, suppose that it is not the case, then there exist $R_{0}, \varepsilon_{0}>0$ such that for all $\delta>0$ there are $u_{\delta}+v_{\delta}, v_{\delta} \in B_{R_{0}}$ with $\left\|v_{\delta}\right\| \leq \delta$ and $C_{2}\|\xi\|_{L^{2}\left(\left(S^{1}\right)^{N}, \mathbb{R}\right)}>\varepsilon_{0}$. Thus, it is possible to obtain for all $n \in \mathbb{N}$ and $\delta=\frac{1}{n}$ functions $u_{n}+v_{n}, v_{n} \in B_{R_{0}}$ such that $\left\|v_{n}\right\| \leq \frac{1}{n}$ and $C_{2}|| \xi_{n} \|_{L^{2}\left(\left(S^{1}\right)^{N}, \mathbb{R}\right)}>\varepsilon_{0}$, for $\xi_{n}(x)=\left|g\left(x, z_{n}(x)\right)-g\left(x, u_{n}(x)\right)\right|$, with $z_{n}=u_{n}+\theta_{n} v_{n}$, and $0 \leq \theta_{n} \leq 1$ depending on $u_{n}$ and $v_{n}$ as before. Since $v_{n} \rightarrow 0$ in $E, v_{n} \rightarrow 0$ in $L^{2}\left(\left(S^{1}\right)^{N}, \mathbb{R}\right)$, $v_{n}(x) \rightarrow 0$ almost everywhere in $\left(S^{1}\right)^{N}$ and there exists $\psi \in L^{2}\left(\left(S^{1}\right)^{N}, \mathbb{R}\right)$ such that $\left|v_{n}(x)\right| \leq$ 
$\psi(x)$ almost everywhere in $\left(S^{1}\right)^{N}$. Furthermore, since $\left(u_{n}\right) \subset B_{R_{0}}$, it is bounded in $E$, then $u_{n} \rightarrow u$ in $E$, up to subsequences. Due to the compact embedding $E \hookrightarrow L^{2}\left(\left(S^{1}\right)^{N}, \mathbb{R}\right), u_{n} \rightarrow u$ in $L^{2}\left(\left(S^{1}\right)^{N}, \mathbb{R}\right)$ up to subsequences, hence $u_{n}(x) \rightarrow u(x)$ almost everywhere in $\left(S^{1}\right)^{N}$ and there exists $\varphi \in L^{2}\left(\left(S^{1}\right)^{N}, \mathbb{R}\right)$ such that $\left|u_{n}(x)\right| \leq \varphi(x)$ almost everywhere in $\left(S^{1}\right)^{N}$ up to subsequences. Thus, $z_{n}(x) \rightarrow u(x)$ almost everywhere in $\left(S^{1}\right)^{N}$, which implies that $\xi_{n}(x) \rightarrow 0$, almost everywhere in $\left(S^{1}\right)^{N}$, provided that $g$ is continuous. Moreover, in view of Remark 2.4 it follows that

$$
\begin{aligned}
\left|\xi_{n}(x)\right|^{2} & \leq 2\left[\left|g\left(x, z_{n}(x)\right)\right|^{2}+\left|g\left(x, u_{n}(x)\right)\right|^{2}\right] \\
& \leq 2\left[C^{2}\left|z_{n}(x)\right|^{2}+C^{2}\left|u_{n}(x)\right|^{2}\right] \\
& \leq 2 C^{2}\left[2\left(\left|u_{n}(x)\right|^{2}+\left|v_{n}(x)\right|^{2}\right)+\left|u_{n}(x)\right|^{2}\right] \\
& \leq 2 C^{2}\left[3\left|u_{n}(x)\right|^{2}+2\left|v_{n}(x)\right|^{2}\right] \\
& \leq 6 C^{2}\left[\varphi^{2}(x)+\psi^{2}(x)\right],
\end{aligned}
$$

almost everywhere in $\left(S^{1}\right)^{N}$. Since $\varphi^{2}+\psi^{2} \in L^{1}\left(\left(S^{1}\right)^{N}, \mathbb{R}\right)$, applying Lebesgue Dominated Convergence Theorem, it yields

$$
\left(\frac{\varepsilon_{0}}{C_{2}}\right)^{2}<\left\|\xi_{n}\right\|_{L^{2}\left(\left(S^{1}\right)^{N}, \mathbb{R}\right)}^{2}=\int_{\left(S^{1}\right)^{N}}\left|\xi_{n}(x)\right|^{2} d x \rightarrow 0,
$$

as $n \rightarrow+\infty$. Which gives a contradiction. Therefore, the result holds.

\subsubsection{Linking Geometry}

To obtain a linking geometry, set $S:=\left(\partial B_{\rho} \cap E_{1}\right)$ and

$$
Q:=\left\{r e+u_{2}: r \geq 0, u_{2} \in E_{2},\left\|r e+u_{2}\right\| \leq r_{1}\right\}
$$

where $0<\rho<r_{1}$ are constants and $e \in E_{1},\|e\|=1$, is chosen suitably. Indeed, due to $\left(G_{3}\right)$ and (2.18), let $e \in E_{1}$ be a unitary eigenvector associated to $\lambda^{+}$, the first positive eigenvalue, then it satisfies

$$
\begin{aligned}
1=\|e\|^{2}=Q_{A}(e) & =\frac{1}{2}(A e, e)_{L^{2}\left(\left(S^{1}\right)^{N}, \mathbb{R}\right)} \\
& =\frac{1}{2} \lambda^{+}\|e\|_{L^{2}\left(\left(S^{1}\right)^{N}, \mathbb{R}\right)}^{2} \\
& <\frac{1}{2} a_{0}\|e\|_{L^{2}\left(\left(S^{1}\right)^{N}, \mathbb{R}\right)}^{2} \\
& \leq \frac{1}{2} \int_{\left(S^{1}\right)^{N}} h(x) e^{2}(x) d x .
\end{aligned}
$$


Choosing such an $e$, it is possible by means of 2.5 to show that for sufficiently large $r_{1}>0$, $\left.I\right|_{S} \geq \alpha>0$ and $\left.I\right|_{\partial Q} \leq 0$ hold, for some $\alpha>0$. Moreover, as noticed before, $S$ and $Q$ "link". Hence, $I$ satisfies $\left(I_{3}\right)$ for some $\alpha>0, \omega=0$ and arbitrary $v \in E_{2}$.

Lemma 2.7. Under hypotheses $\left(V_{1}^{\prime}\right)-\left(V_{2}^{\prime}\right)$ on $V$ and $\left(G_{1}\right)-\left(G_{3}\right)$ on $g$, I satisfies $\left(I_{3}\right)$.

Proof. Note that from $\left(G_{1}\right)-\left(G_{2}\right)$, given $\varepsilon>0$ and $2<p<2^{*}$, there exists $C_{\varepsilon}>0$ such that

$$
|G(x, s)| \leq \frac{\varepsilon}{2}|s|^{2}+\frac{C_{\varepsilon}}{p}|s|^{p}
$$

and

$$
|g(x, s)| \leq \varepsilon|s|+C_{\varepsilon}|s|^{p-1},
$$

for all $s \in \mathbb{R}$, and for all $x \in \mathbb{R}^{N}$. Since $S \subset E_{1}$, for all $u_{1} \in S$, it yields

$$
\begin{aligned}
I\left(u_{1}\right) & =\frac{1}{2}\left\|u_{1}\right\|^{2}-\int_{\left(S^{1}\right)^{N}} G\left(x, u_{1}(x)\right) d x \\
& \geq \frac{1}{2} \rho^{2}-\int_{\left(S^{1}\right)^{N}}\left(\frac{\varepsilon}{2}\left|u_{1}(x)\right|^{2}+\frac{C_{\varepsilon}}{p}\left|u_{1}(x)\right|^{p}\right) d x \\
& \geq \frac{1}{2} \rho^{2}-\left(\frac{\varepsilon}{2} C_{2}^{2}\left\|u_{1}\right\|^{2}+\frac{C_{\varepsilon}}{p} C_{p}^{p}\left\|u_{1}\right\|^{p}\right) \\
& =\rho^{2}\left[\frac{1}{2}\left(1-\varepsilon C_{2}^{2}\right)-\frac{C_{\varepsilon}}{p} C_{p}^{p} \rho^{p-2}\right] \\
& \geq \rho^{2}\left(d_{1}-d_{2}\right)=\alpha>0,
\end{aligned}
$$

where $\varepsilon, \rho$ are sufficiently small, such that $1>\varepsilon C_{2}^{2}$ and also

$$
d_{1}:=\frac{1}{2}\left(1-\varepsilon C_{2}^{2}\right)>\frac{C_{\varepsilon}}{p} C_{p}^{p} \rho^{p-2}=: d_{2} .
$$

Therefore, from (2.22), $\left(I_{3}\right)(i)$ holds for $I$.

So as to ensure that $I$ satisfies $\left(I_{3}\right)(i i)$ in Theorem 1.4 , with $\omega=0$, observe that $I(u) \leq 0$, for all $u \in E_{2}=E^{-} \oplus E^{0}$, then it suffices to show that $I(r e+u) \leq 0$ for $r>0, u \in E_{2}$ and $\|r e+u\| \geq r_{1}$, for some $r_{1}>0$ large enough. Arguing indirectly assume that for some sequence $\left(r_{n} e+u_{n}\right) \subset \mathbb{R}^{+} e \oplus E_{2}$ with $\left\|r_{n} e+u_{n}\right\| \rightarrow+\infty, I\left(r_{n} e+u_{n}\right)>0$ holds, for all $n \in \mathbb{N}$. Seeking a contradiction, set

$$
\tilde{u}_{n}:=\frac{r_{n} e+u_{n}}{\left\|r_{n} e+u_{n}\right\|}=s_{n} e+w_{n}
$$

where $s_{n} \in \mathbb{R}^{+}, w_{n}=w_{n}^{-}+w_{n}^{0} \in E_{2}=E^{-} \oplus E^{0}$ and $\left\|\tilde{u}_{n}\right\|=1$. Provided that $\left(\tilde{u}_{n}\right)$ is bounded, up to subsequences it follows that $\tilde{u}_{n} \rightarrow \tilde{u}=s e+w$ in $E$. Then, up to subsequences, $\tilde{u}_{n}(x) \rightarrow \tilde{u}(x)$ almost everywhere in $\mathbb{R}^{N}, s_{n} \rightarrow s$ in $\mathbb{R}^{+}, w_{n}^{-} \rightarrow w$ in $E$, and $w_{n}^{0} \rightarrow w^{0}$ in $E$, since $\left(w_{n}^{0}\right) \subset E^{0}$ and $E^{0}$ is finite dimensional. Seeing that $1=\left\|s_{n} e+w_{n}\right\|^{2}=s_{n}^{2}+\left\|w_{n}^{-}\right\|^{2}+\left\|w_{n}^{0}\right\|^{2}$, it follows 
that $0 \leq s_{n}^{2} \leq 1$, and it yields

$$
\begin{aligned}
\frac{I\left(r_{n} e+u_{n}\right)}{\left\|r_{n} e+u_{n}\right\|^{2}} & =s_{n}^{2}\|e\|^{2}-\left\|w_{n}^{-}\right\|^{2}-\int_{\left(S^{1}\right)^{N}} \frac{G\left(x, r_{n} e(x)+u_{n}(x)\right)}{\left\|r_{n} e+u_{n}\right\|^{2}} d x \\
& =2 s_{n}^{2}-1-\left\|w_{n}^{0}\right\|^{2}-\int_{\left(S^{1}\right)^{N}} \frac{G\left(x, r_{n} e(x)+u_{n}(x)\right)}{\left\|r_{n} e+u_{n}\right\|^{2}} d x>0,
\end{aligned}
$$

hence $\frac{1}{2} \leq s^{2} \leq 1$. Moreover, from 2.21 it follows that $1<\frac{1}{2} \int_{\left(S^{1}\right)^{N}} h(x) e^{2}(x) d x$. Then,

$$
\begin{aligned}
0 & >s^{2}-s^{2} \frac{1}{2} \int_{\left(S^{1}\right)^{N}} h(x) e^{2}(x) d x \\
& \geq s^{2}\left(1-\frac{1}{2} \int_{\left(S^{1}\right)^{N}} h(x) e^{2}(x) d x\right)-\left(1+\left\|w^{0}\right\|^{2}-s^{2}\right)-\frac{1}{2} \int_{\left(S^{1}\right)^{N}} h(x) w^{2}(x) d x \\
& =s^{2}\left(2-\frac{1}{2} \int_{\left(S^{1}\right)^{N}} h(x) e^{2}(x) d x\right)-1-\left\|w^{0}\right\|^{2}-\frac{1}{2} \int_{\left(S^{1}\right)^{N}} h(x) w^{2}(x) d x .
\end{aligned}
$$

On the other hand, from assumptions $\left(G_{1}\right)-\left(G_{2}\right)$ and since $\tilde{u}_{n}$ is convergent in $L^{2}\left(\left(S^{1}\right)^{N}, \mathbb{R}\right)$, there exists some $\psi \in L^{1}\left(\left(S^{1}\right)^{N}, \mathbb{R}\right)$ such that

$$
\left|\frac{G\left(\cdot, r_{n} e(\cdot)+u_{n}(\cdot)\right)}{|| r_{n} e+u_{n} \|^{2}}\right| \leq r_{\infty}\left|\tilde{u}_{n}(\cdot)\right|^{2} \leq \psi(\cdot) \in L^{1}\left(\left(S^{1}\right)^{N}, \mathbb{R}\right) .
$$

Moreover, provided that $\left\|r_{n} e+u_{n}\right\| \rightarrow+\infty$, and $\tilde{u}_{n}(x) \rightarrow \tilde{u}(x) \neq 0$, almost everywhere in $\operatorname{supp}(\tilde{u})$, it follows that $u_{n}(x)=\tilde{u}_{n}(x)\left\|r_{n} e+u_{n}(x)\right\| \rightarrow+\infty$ almost everywhere in $\operatorname{supp}(\tilde{u})$, as $n \rightarrow+\infty$, hence

$$
\frac{G\left(x, r_{n} e(x)+u_{n}(x)\right)}{\left\|r_{n} e+u_{n}\right\|^{2}}=\frac{G\left(x, \tilde{u}_{n}(x)\left\|r_{n} e+u_{n}\right\|\right) \tilde{u}_{n}^{2}(x)}{\tilde{u}_{n}^{2}(x)\left\|r_{n} e+u_{n}\right\|^{2}} \rightarrow \frac{1}{2} h(x) \tilde{u}^{2}(x)
$$

almost everywhere in $\operatorname{supp}(\tilde{z}) \cap S^{1}$ as $n \rightarrow+\infty$. Note that, $\operatorname{supp}(\tilde{u}) \neq \emptyset$, because $\tilde{u}=s e+w$, with $\operatorname{supp}(e) \neq \emptyset$ and $(e, w)_{L^{2}\left(\left(S^{1}\right)^{N}, \mathbb{R}\right)}=0$. Thus, by Lebesgue Dominated Convergence Theorem,

$$
\int_{\left(S^{1}\right)^{N}} \frac{G\left(x, r_{n} e(x)+u_{n}(x)\right)}{\left\|r_{n} e+u_{n}\right\|^{2}} d x \rightarrow \frac{1}{2} \int_{\left(S^{1}\right)^{N}} h(x)(s e(x)+w(x))^{2} d x,
$$

as $n \rightarrow+\infty$. Passing to the limit in 2.23 as $n \rightarrow+\infty$, it yields

$$
\begin{aligned}
0 & \leq 2 s^{2}-1-\left\|w^{0}\right\|^{2}-\frac{1}{2} \int_{\left(S^{1}\right)^{N}} h(x)\left(s^{2} e^{2}(x)+w^{2}(x)\right) d x \\
& =s^{2}\left(2-\frac{1}{2} \int_{\left(S^{1}\right)^{N}} h(x) e^{2}(x) d x\right)-1-\left\|w^{0}\right\|^{2}-\frac{1}{2} \int_{\left(S^{1}\right)^{N}} h(x) w^{2}(x) d x
\end{aligned}
$$


which is contrary to 2.24). Therefore the result holds.

\subsubsection{Boundedness of Cerami Sequences}

Now, last condition of Theorem 1.4 is proved, and lastly the main result of this section is demonstrated.

Lemma 2.8. Suppose that $V$ satisfies $\left(V_{1}^{\prime}\right)-\left(V_{2}^{\prime}\right)$ and $g$ satisfies $\left(G_{1}\right)-\left(G_{4}\right)$, then I satisfies $\left(I_{4}\right)$.

Proof. Let $b>0$ be an arbitrary constant, and take $\left(u_{n}\right) \subset I^{-1}([c-b, c+b])$ such that $\left(1+\left\|u_{n}\right\|\right)\left\|I^{\prime}\left(u_{n}\right)\right\| \rightarrow 0$. Then $\left(u_{n}\right)$ is bounded. In fact, suppose by contradiction that $\left\|u_{n}\right\| \rightarrow+\infty$, up to subsequences. Setting $\tilde{u}_{n}:=\frac{u_{n}}{\left\|u_{n}\right\|}$, it is bounded, hence $\tilde{u}_{n} \rightarrow \tilde{u}$ in $E$ and $\tilde{u}_{n} \rightarrow \tilde{u}$ in $L^{\beta}\left(\left(S^{1}\right)^{N}, \mathbb{R}\right)$, for $\beta \in[1,+\infty)$, due to the compact embeddings previously mentioned. Writing $u_{n}=u_{n}^{+}+u_{n}^{-}+u_{n}^{0} \in E^{+} \oplus E^{-} \oplus E^{0}$, by the choice of $u_{n}$ it satisfies

$$
\begin{aligned}
o_{n}(1) & =I^{\prime}\left(u_{n}\right) \frac{u_{n}^{+}}{\left\|u_{n}\right\|^{2}} \\
& =\frac{1}{\left\|u_{n}\right\|} I^{\prime}\left(u_{n}\right) \tilde{u}_{n}^{+} \\
& =\left\|\tilde{u}_{n}^{+}\right\|^{2}-\int_{\left(S^{1}\right)^{N}} \frac{g\left(x, u_{n}(x)\right)}{u_{n}(x)} \tilde{u}_{n}(x) \tilde{u}_{n}^{+}(x) d x .
\end{aligned}
$$

and

$$
\begin{aligned}
o_{n}(1) & =I^{\prime}\left(u_{n}\right) \frac{u_{n}^{-}}{\left\|u_{n}\right\|^{2}} \\
& =\frac{1}{\left\|u_{n}\right\|} I^{\prime}\left(u_{n}\right) \tilde{u}_{n}^{-} \\
& =-\left\|\tilde{u}_{n}^{-}\right\|^{2}-\int_{\left(S^{1}\right)^{N}} \frac{g\left(x, z_{n}(x)\right)}{u_{n}(x)} \tilde{u}_{n}(x) \tilde{u}_{n}^{-}(x) d x .
\end{aligned}
$$

Subtracting 2.27) from 2.26), and using that $1=\left\|\tilde{u}_{n}^{+}\right\|^{2}+\left\|\tilde{u}_{n}^{-}\right\|^{2}+\left\|\tilde{u}_{n}^{0}\right\|^{2}$, it yields

$$
o_{n}(1)=1-\left\|\tilde{u}_{n}^{0}\right\|^{2}-\int_{\left(S^{1}\right)^{N}} \frac{g\left(x, u_{n}(x)\right)}{u_{n}(x)}\left[\left(\tilde{u}_{n}^{+}(x)\right)^{2}-\left(\tilde{u}_{n}^{-}(x)\right)^{2}\right] d x .
$$

Provided that $\left(\tilde{u}_{n}^{0}\right) \subset E^{0}$, which is finite dimensional, then weak convergence implies that $\tilde{u}_{n}^{0} \rightarrow \tilde{u}^{0}$ in $E$. Furthermore, since $\tilde{u}_{n} \rightarrow \tilde{u}$ in $L^{2}\left(\left(S^{1}\right)^{N}, \mathbb{R}\right)$, there exist $\psi^{+}, \psi^{-} \in L^{2}\left(\left(S^{1}\right)^{N}, \mathbb{R}\right)$ such that $\left|\tilde{u}_{n}^{+}(x)\right| \leq \psi^{+}(x)$ and $\left|\tilde{u}_{n}^{-}(x)\right| \leq \psi^{-}(x)$, almost everywhere in $\mathbb{R}^{N}$, hence from Remark 2.4 it follows that

$$
\left|\frac{g\left(\cdot, u_{n}(\cdot)\right)}{u_{n}(\cdot)}\left[\left(\tilde{u}_{n}^{+}(\cdot)\right)^{2}-\left(\tilde{u}_{n}^{-}(\cdot)\right)^{2}\right]\right| \leq C\left[\left(\psi^{+}(\cdot)\right)^{2}+\left(\psi^{-}(\cdot)\right)^{2}\right] \in L^{1}\left(\left(S^{1}\right)^{N}, \mathbb{R}\right) .
$$


Since $\tilde{u}_{n} \rightarrow \tilde{u}$ in $L^{2}\left(\left(S^{1}\right)^{N}, \mathbb{R}\right), u_{n}(x) \rightarrow+\infty$, for all $x$ such that $\tilde{u}(x) \neq 0$, from $\left(G_{2}\right)$ it follows that

$$
\begin{aligned}
\frac{g\left(x, u_{n}(x)\right)}{u_{n}(x)}\left[\left(\tilde{u}_{n}^{+}(x)\right)^{2}-\left(\tilde{u}_{n}^{-}(x)\right)^{2}\right] & =\left(\frac{h(x) \tilde{u}_{n}(x)}{\tilde{u}_{n}(x)}+\frac{\tilde{g}\left(x, u_{n}(x)\right)}{u_{n}(x)}\right)\left[\left(\tilde{u}_{n}^{+}(x)\right)^{2}-\left(\tilde{u}_{n}^{-}(x)\right)^{2}\right] \\
& \rightarrow h(x)\left[\left(\tilde{u}^{+}(x)\right)^{2}-\left(\tilde{u}^{-}(x)\right)^{2}\right],
\end{aligned}
$$

as $n \rightarrow+\infty$. Therefore, by Lebesgue Dominated Convergence Theorem, passing to the limit in 2.28 as $n \rightarrow+\infty$, it yields

$$
\int_{\left(S^{1}\right)^{N}} h(x)\left[\left(\tilde{u}^{+}(x)\right)^{2}-\left(\tilde{u}^{-}(x)\right)^{2}\right] d x=1-\left\|\tilde{u}^{0}\right\|^{2} .
$$

On the other hand, given $v \in E$, from $\left(G_{2}\right)$ it follows that

$$
\int_{\left(S^{1}\right)^{N}} \frac{g\left(x, u_{n}(x)\right)}{u_{n}(x)} \tilde{u}_{n}(x) v(x) d x=\int_{\left(S^{1}\right)^{N}} h(x) \tilde{u}_{n}(x) v(x) d x+\int_{\left(S^{1}\right)^{N}} \frac{\tilde{g}\left(x, u_{n}(x)\right)}{u_{n}(x)} \tilde{u}_{n}(x) v(x) d x .
$$

In virtue of the same arguments as above it follows that

$$
\int_{\left(S^{1}\right)^{N}} \frac{g\left(x, u_{n}(x)\right)}{u_{n}(x)} \tilde{u}_{n}(x) v(x) d x=\int_{\left(S^{1}\right)^{N}} h(x) \tilde{u}_{n}(x) v(x) d x+o_{n}(1),
$$

as $n \rightarrow+\infty$. Hence, using the same arguments as above and applying Lebesgue Dominated Convergence Theorem, it yields

$$
\begin{aligned}
o_{n}(1) & =\frac{I^{\prime}\left(u_{n}\right) v}{\left\|u_{n}\right\|} \\
& =\frac{Q_{A}^{\prime}\left(u_{n}\right) v}{\left\|u_{n}\right\|}-\int_{\left(S^{1}\right)^{N}} \frac{g\left(x, u_{n}(x)\right)}{u_{n}(x)} \tilde{u}_{n}(x) v(x) d x \\
& =\left(A \tilde{u}_{n}, v\right)_{L^{2}\left(\left(S^{1}\right)^{N}, \mathbb{R}\right)}-\int_{\left(S^{1}\right)^{N}} h(x) \tilde{u}_{n}(x) v(x) d x+o_{n}(1) \\
& =\left(\mathscr{O} \tilde{u}_{n}, v\right)_{L^{2}\left(\left(S^{1}\right)^{N}, \mathbb{R}\right)}+o_{n}(1) \\
& =(\mathscr{O} \tilde{u}, v)_{L^{2}\left(\left(S^{1}\right)^{N}, \mathbb{R}\right)}+o_{n}(1) .
\end{aligned}
$$

Due to 2.30), if $\tilde{u} \neq 0$, it is an eigenvector of $\mathscr{O}$, with eigenvalue 0 . Nevertheless, from $\left(G_{4}\right)$, $0 \notin \sigma_{p}(\mathscr{O})$ and hence, $\tilde{u}=0$. It means that $\tilde{u}^{+}=\tilde{u}^{-}=\tilde{u}^{0}=0$ and thus, 2.29 yields another contradiction. Therefore, the result holds.

Finally it is possible to prove the main result of this section.

Proof of Theorem 2.2. Provided that $I$ satisfies all assumptions $\left(I_{1}\right)-\left(I_{4}\right)$ in Theorem 1.4 , ap-

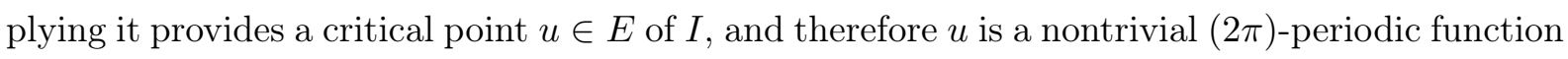


in $x_{i}, i=1, \ldots, N$, and a weak solution to $(H S)$, since $I(u)=c>0$, hence $u \not \equiv 0$.

Next section, two nonlinear Schrödinger equations are studied and nontrivial weak solutions are found by applying the abstract result obtained in Chapter 2 .

\subsection{Asymptotically Linear Schrödinger Equations in $\mathbb{R}^{N}$ via an Abstract Linking Theorem}

This section introduce two applications for the abstract critical point theorem developed in Chapter 2. The main difference between them is how to obtain the linking geometry, based on their spectra, which are very different to each other.

First, consider problem $(P)$

$$
-\Delta u+V(x) u=g(x, u) \text { in } \mathbb{R}^{N},
$$

for $N \geq 3$, where potential $V$ satisfies:

$\left(V_{1}\right) \quad V \in C\left(\mathbb{R}^{N}, \mathbb{R}\right)$ and $\lim _{|x| \rightarrow+\infty} V(x)=V_{\infty}>0 ;$

$\left(V_{2}\right)$ Setting $A:=-\Delta+V(x)$, a self-adjoint operator of $L^{2}\left(\mathbb{R}^{N}\right)$,

$$
\sup [\sigma(A) \cap(-\infty, 0)]=\sigma^{-}<0<\sigma^{+}=\inf [\sigma(A) \cap(0,+\infty)]
$$

Remark 2.5. In view of $\left(V_{1}\right), V(x)$ is bounded and $\sigma_{\text {ess }}(A)=\left[V_{\infty},+\infty\right) \subset(0,+\infty)$ (cf. [52] Theorem 3.15, page 44), hence $\sigma(A) \cap\left(-\infty, V_{\infty}\right)=\sigma_{d}(A) \cap\left(-\infty, V_{\infty}\right)$. Furthermore, hypothesis $\left(V_{2}\right)$ implies that either $0 \notin \sigma(A)$ or $0 \in \sigma_{d}(A)$, since $0 \in\left(\sigma^{-}, \sigma^{+}\right)$is an isolated point and the essential spectrum $\left[V_{\infty},+\infty\right)$ does not have isolated points, hence $0 \notin \sigma_{\text {ess }}(A)=\left[V_{\infty},+\infty\right)$ which implies that $V_{\infty}>0$, namely $V_{\infty}$ is positive, therefore assumption $V_{\infty}>0$ in $\left(V_{1}\right)$ is redundant. With this in hand, it is possible to introduce by means of operator $A$ an equivalent norm $\|\cdot\|$ to the usual norm $\|\cdot\|_{H^{1}\left(\mathbb{R}^{N}\right)}$, in $H^{1}\left(\mathbb{R}^{N}\right)$ (cf. [17], Lemma 1.2). Thus, space $E=\left(H^{1}\left(\mathbb{R}^{N}\right),\|\cdot\|\right)$, will be the Hilbert space used in order to apply Theorem 1.4. Finally, $\left(V_{2}\right)$ implies that $\emptyset \neq \sigma(A) \cap(-\infty, 0)=\sigma_{d}(A) \cap(-\infty, 0)$, i. e., operator $A$ has negative eigenvalues. Furthermore, this set is finite (cf. [23] Theorem 30, page 150). An example satisfying $\left(V_{1}\right)-\left(V_{2}\right)$ is given by a continuous $V(x)$ such that

$$
V(x)=\left\{\begin{array}{cc}
-V_{0} & |x|<R \\
V_{\infty} & |x|>2 R
\end{array}\right.
$$

where $V_{0}>\frac{\lambda_{1}(1)}{R^{2}}>0$ is a constant and $\lambda_{1}(1)$ is the first eigenvalue of the operator $\left(-\Delta, H_{0}^{1}\left(B_{1}(0)\right)\right)$. 
In fact, if $\psi$ is an eigenfunction associated to $\lambda_{1}(R)=\frac{\lambda_{1}(1)}{R^{2}}$, which is the first eigenvalue of the operator $\left(-\Delta, H_{0}^{1}\left(B_{R}(0)\right)\right)$, it yields

$$
\begin{aligned}
(A \psi, \psi)_{L^{2}\left(\mathbb{R}^{N}\right)} & =\int_{B_{1}(R)}|\nabla \psi(x)|^{2}+V(x) \psi(x)^{2} d x \\
& \leq\left(\lambda_{1}(R)-V_{0}\right)\|\psi\|_{L^{2}\left(B_{R}(0)\right)}^{2} \\
& =\left(\frac{\lambda_{1}(1)}{R^{2}}-V_{0}\right)\|\psi\|_{L^{2}\left(\mathbb{R}^{N}\right)}^{2} \\
& <-\varepsilon\|\psi\|_{L^{2}\left(\mathbb{R}^{N}\right)}^{2}
\end{aligned}
$$

for some $\varepsilon>0$, which implies the bottom of the spectrum of $A$ is negative.

Henceforth consider the case where $g(x, s)=h(x) f(s)$, and $h$ satisfies

$\left(h_{1}\right) h \in C\left(\mathbb{R}^{N},(0,+\infty)\right)$ and $\lim _{|x| \rightarrow+\infty} h(x)=0 ;$

$\left(h_{2}\right) h \in L^{q}\left(\mathbb{R}^{N}\right), q=\frac{2^{*}}{2^{*}-p}$, for some $p \in\left(2,2^{*}\right)$.

Furthermore, $f$ is asymptotically linear satisfying

$\left(f_{1}\right) \quad f \in C(\mathbb{R}, \mathbb{R})$ and $\lim _{s \rightarrow 0} \frac{f(s)}{s}=0 ;$

$\left(f_{2}\right)$ There exists $a>0$ such that $\lim _{s \rightarrow+\infty} \frac{F(s)}{s^{2}}=\frac{a}{2}$, where $F(s):=\int_{0}^{s} f(t) d t$, and $F(s) \geq 0$.

$\left(f_{3}\right)$ Setting $Q(s):=\frac{1}{2} f(s) s-F(s)>0$ for all $s \in \mathbb{R} \backslash\{0\}$,

$$
\lim _{s \rightarrow+\infty} Q(s)=+\infty
$$

Remark 2.6. Observe that assumptions $\left(h_{1}\right)-\left(h_{2}\right)$ give that $h \in L^{\infty}\left(\mathbb{R}^{N}\right)$ and $\|h\|_{\infty}=h_{\infty}>0$. In addition, $\left(f_{2}\right)$ implies that $\lim _{s \rightarrow+\infty} F(s)=+\infty$, hence by $\left(f_{2}\right)$ and L'Hospital rule, it follows that $\lim _{s \rightarrow+\infty} \frac{f(s)}{s}=a$. Moreover, due to assumptions $\left(f_{1}\right)-\left(f_{2}\right)$ there exists $\kappa>0$ such that $|f(s)| \leq \kappa|s|$ for all $s \in \mathbb{R}$ and $a \leq \kappa$. An example satisfying $\left(f_{1}\right)-\left(f_{3}\right)$ but not with $\frac{f(s)}{s}$ increasing, is a continuous $f(s)$ such that

$$
f(s)=\left\{\begin{array}{cl}
\frac{s^{7}-\frac{3}{2} s^{5}+2 s^{3}}{1+s^{6}} & |s|<5 \\
\frac{s^{3}}{1+s^{2}} & |s|>10 .
\end{array}\right.
$$




\subsubsection{Variational Setting}

Let $I: E \rightarrow \mathbb{R}$ be the energy functional associated to problem $(P)$ in $(2.31)$, which is given by

$$
I(u)=\frac{1}{2} \int_{\mathbb{R}^{N}}\left(|\nabla u|^{2}+V(x) u^{2}\right)-\int_{\mathbb{R}^{N}} h(x) F(u(x)) d x,
$$

for all $u \in E$. As observed in Remark 2.5, the set of eigenvalues $\sigma_{d}(A) \cap(-\infty, 0)$ is finite, then one can denote it by $\left\{\lambda_{i}\right\}_{i=1}^{j}$, for some $j \in \mathbb{N}$, counting multiplicities, and in addition, denote by $\varphi_{i} \in E$ the eigenfunction associated to $\lambda_{i}$, for $i=1, \ldots, j$ and then set $E^{-}:=\operatorname{span}\left\{\varphi_{i}\right\}_{i=1}^{j}$. Moreover, setting $E^{0}:=\operatorname{ker}(A)$, if $0 \notin \sigma(A)$, then $E^{0}=\{0\}$, if not, then $0 \in \sigma_{d}(A)$, hence $E^{0}$ is finite dimensional. Thus, $E^{-} \oplus E^{0}$, it is a finite dimensional subspace of $E$ and setting $E^{+}:=\left(E^{-} \oplus E^{0}\right)^{\perp}$, it is the subspace of $E$ in which operator $A$ is positive definite. With this, $E=E^{+} \oplus E^{-} \oplus E^{0}$ and every function $u \in E$ can be uniquely written as $u=u^{+}+u^{-}+u^{0}$, with $u^{+} \in E^{+}, u^{0} \in E^{0}$ and $u^{-} \in E^{-}$. Furthermore, as in [17] operator $A$ induce an equivalent norm $\|\cdot\|$ to the standard $H^{1}\left(\mathbb{R}^{N}\right)$-norm and a corresponding inner product $(\cdot, \cdot)$ in $E$ given by

$$
\|u\|^{2}:=\left(A u^{+}, u^{+}\right)_{L^{2}\left(\mathbb{R}^{N}\right)}-\left(A u^{-}, u^{-}\right)_{L^{2}\left(\mathbb{R}^{N}\right)}+\left\|u^{0}\right\|_{L^{2}\left(\mathbb{R}^{N}\right)}^{2},
$$

and

$$
(u, v):=\left\{\begin{array}{lr}
\int_{\mathbb{R}^{N}}(\nabla u(x) \nabla v(x)+V(x) u(x) v(x)) d x=(A u, v)_{L^{2}\left(\mathbb{R}^{N}\right)} & \text { if } u, v \in E^{+}, \\
-\int_{\mathbb{R}^{N}}(\nabla u(x) \nabla v(x)+V(x) u(x) v(x)) d x=-(A u, v)_{L^{2}\left(\mathbb{R}^{N}\right)} & \text { if } u, v \in E^{-}, \\
(u, v)_{L^{2}\left(\mathbb{R}^{N}\right)} & \text { if } u, v \in E^{0}, \\
0 & \text { if } u \in E^{j}, v \in E^{k}, j \neq k,
\end{array}\right.
$$

for $j, k \in\{+,-, 0\}$. Henceforth, the Hilbert space used in this application is $E=\left(H^{1}\left(\mathbb{R}^{N}\right),\|\cdot\|\right)$ and in addition, it is possible to write

$$
I(u)=\frac{1}{2}\left(\left\|u^{+}\right\|^{2}-\left\|u^{-}\right\|^{2}\right)-\int_{\mathbb{R}^{N}} h(x) F(u(x)) d x,
$$

for all $u=u^{+}+u^{-}+u^{0} \in E$. Note that the orthogonality among $E^{+}, E^{-}, E^{0}$, ensures that $u^{+}, u^{-}, u^{0}$ are also orthogonal in $L^{2}\left(\mathbb{R}^{\mathbb{N}}\right)$. As usual, $\|u\|_{L^{2}\left(\mathbb{R}^{N}\right)}^{2}=(u, u)_{L^{2}\left(\mathbb{R}^{N}\right)}$ denotes the norm and inner product in $L^{2}\left(\mathbb{R}^{\mathbb{N}}\right)$, then $\left(u^{j}, u^{k}\right)_{L^{2}\left(\mathbb{R}^{N}\right)}=0, j \neq k$ and $j, k \in\{+,-, 0\}$.

Denoting by $\{\mathscr{E}(\lambda)\}$ the spectral family of operator $A$, in view of Spectral Theory (cf. [9], Supplement S1.1 page 386; see also [39] Chapter 3) it is possible to define $E_{2}:=\mathscr{E}(0) E=E^{-} \oplus E^{0}$ and $E_{1}:=(I-\mathscr{E}(0)) E$. Furthermore, $\mathscr{E}(0)=\mathscr{E}(\lambda)$, for all $0<\lambda<\sigma^{+}$, 
by the definition of $\sigma^{+}$in $\left(V_{2}\right)$, then $E_{1}=(I-\mathscr{E}(\lambda)) E$, for all $0<\lambda<\sigma^{+}$. Hence, by [9] (cf. Theorem 1.1', page 394) it follows that $\sigma^{+}\left\|u_{1}\right\|_{L^{2}\left(\mathbb{R}^{N}\right)}^{2} \leq\left\|u_{1}\right\|^{2}$, for all $u_{1} \in E_{1}$. Therefore,

$$
\inf _{u_{1} \in E_{1}, u_{1} \neq 0} \frac{\left\|u_{1}\right\|^{2}}{\left\|u_{1}\right\|_{L^{2}\left(\mathbb{R}^{N}\right)}^{2}} \geq \sigma^{+}
$$

and then, setting

$$
a_{0}:=\inf _{u_{1} \in E_{1}, u_{1} \neq 0} \frac{\left\|u_{1}\right\|^{2}}{\left\|h^{\frac{1}{2}} u_{1}\right\|_{L^{2}\left(\mathbb{R}^{N}\right)}^{2}} \geq \frac{1}{h_{\infty}} \inf _{u_{1} \in E_{1}, u_{1} \neq 0} \frac{\left\|u_{1}\right\|^{2}}{\left\|u_{1}\right\|_{L^{2}\left(\mathbb{R}^{N}\right)}^{2}} \geq \frac{\sigma^{+}}{h_{\infty}}>0,
$$

it follows that

$$
\left\|u_{1}\right\|^{2} \geq a_{0} \int_{\mathbb{R}^{N}} h(x) u_{1}^{2}(x) d x
$$

for all $u_{1} \in E_{1}$.

Under all previous assumptions and notations, it is possible to state the first main result of this section.

Theorem 2.3. Assume $V$ satisfying $\left(V_{1}\right)-\left(V_{2}\right)$, $h$ satisfying $\left(h_{1}\right)-\left(h_{2}\right)$ and $f$ satisfying $\left(f_{1}\right)-$ $\left(f_{3}\right)$, with $a>a_{0}$. Then problem $(P)$ has a nontrivial weak solution $u \in H^{1}\left(\mathbb{R}^{N}\right)$.

\subsubsection{Boundedness of Cerami Sequences}

In order to prove Theorem 2.3, first the boundedness of Cerami sequences of $I$ defined in (2.32) is going to be showed. Subsequently, hypotheses $\left(I_{1}\right)-\left(I_{4}\right)$ in Theorem 1.4 will be checked, and then will be possible to ensure the result by applying the theorem concerned. For now, observe that by $\left(f_{1}\right)$ and $\left(f_{2}\right)$, given $\varepsilon>0$ and $2<p<2^{*}$ there exists a constant $C_{\varepsilon}>0$ such that

$$
|F(s)| \leq \frac{\varepsilon}{2}|s|^{2}+\frac{C_{\varepsilon}}{p}|s|^{p}
$$

and

$$
|f(s)| \leq \varepsilon|s|+C_{\varepsilon}|s|^{p-1}
$$

for all $s \in \mathbb{R}$.

Recall that given $\left(v_{n}\right)$ a bounded sequence in $H^{1}\left(\mathbb{R}^{N}\right)$, up to subsequences, $\left(v_{n}\right)$ satisfies exactly one of the cases below:

(i) Vanishing: for all $r>0$,

$$
\limsup _{n \rightarrow+\infty} \sup _{y \in \mathbb{R}^{N}} \int_{B_{r}(y)}\left|u_{n}(x)\right|^{2} d x=0 .
$$


(ii) Non-vanishing: there exist, $r, \varrho>0$ and a sequence $\left(y_{n}\right) \in \mathbb{R}^{N}$ such that

$$
\limsup _{n \rightarrow+\infty} \int_{B_{r}\left(y_{n}\right)}\left|u_{n}(x)\right|^{2} d x>\varrho .
$$

Lemma 2.9. Assume that $\left(V_{1}\right)-\left(V_{2}\right),\left(h_{1}\right)-\left(h_{2}\right)$ and $\left(f_{1}\right)-\left(f_{3}\right)$ hold for $I$ and let $\left(u_{n}\right) \subset E$ be a Cerami sequence of $I$ in an arbitrary level $c \in \mathbb{R}$, then $\left(u_{n}\right)$ is bounded.

Proof. Suppose by contradiction that, up to subsequences, $\left\|u_{n}\right\| \rightarrow+\infty$ as $n \rightarrow+\infty$. Defining $v_{n}:=\frac{u_{n}}{\left\|u_{n}\right\|}$ it follows that $\left(v_{n}\right)$ is a bounded sequence in $E$. Then $v_{n} \rightarrow v$ as $n \rightarrow+\infty$, up to subsequences. Since $\left(v_{n}\right)$ is bounded it satisfies one of the cases, either vanishing or nonvanishing. The idea is to show that neither vanishing, nor non-vanishing can occur for $\left(v_{n}\right)$, which yields a contradiction and proves that $\left(u_{n}\right)$ is bounded.

Firstly, suppose that non-vanishing case holds for $\left(v_{n}\right)$, up to subsequences and let $\left(y_{n}\right) \subset \mathbb{R}^{N}$ be a sequence given by $(i i)$. Whereas $\left(u_{n}\right)$ is a Cerami sequence, given $\phi \in C_{0}^{\infty}\left(\mathbb{R}^{N}\right)$ set $\phi_{n}(x):=\phi\left(x-y_{n}\right)$, then $\left\|\phi_{n}\right\|_{H^{1}\left(\mathbb{R}^{N}\right)}=\|\phi\|_{H^{1}\left(\mathbb{R}^{N}\right)}$ and

$$
\left|I^{\prime}\left(u_{n}\right) \phi_{n}\right| \leq\left\|I^{\prime}\left(u_{n}\right)\right\|_{E^{*}}\left\|\phi_{n}\right\|_{E} \leq\left. c_{1}\left\|I^{\prime}\left(u_{n}\right)\right\|\right|_{E^{*}}\|\phi\|_{H_{0}^{1}\left(\mathbb{R}^{N}\right)} \leq c_{2}\left\|I^{\prime}\left(u_{n}\right)\right\|\|\phi\|_{E} \rightarrow 0,
$$

as $n \rightarrow+\infty$, where $c_{1}, c_{2}$ are positive constants such that $\|w\|_{E} \leq c_{1}\|w\|_{H_{0}^{1}\left(\mathbb{R}^{N}\right)} \leq c_{2}\|w\|_{E}$, for all $w \in E$, by the equivalence of norms. Since $\left\|u_{n}\right\| \rightarrow+\infty$, setting

$$
\Omega_{n}:=\left\{x \in \mathbb{R}^{N}:\left|u_{n}(x)\right| \neq 0\right\},
$$

it follows that the Lebesgue measure is $\mu\left(\Omega_{n}\right)>0$, therefore putting $v_{n}=v_{+, n}+v_{-, n}+v_{0, n}$, where $v_{i, n} \in E^{j}, j=+,-, 0$ it yields

$$
\begin{aligned}
o_{n}(1) & =\frac{1}{\left\|u_{n}\right\|} I^{\prime}\left(u_{n}\right) \phi_{n} \\
& =\left(v_{+, n}-v_{-, n}, \phi_{n}\right)-\int_{\mathbb{R}^{N}} h(x) \frac{f\left(u_{n}(x)\right)}{\left\|u_{n}\right\|} \phi_{n}(x) d x \\
& =\left(v_{+, n}-v_{-, n}, \phi_{n}\right)-\int_{\Omega_{n}} h(x) \frac{f\left(u_{n}(x)\right)}{u_{n}(x)} v_{n}(x) \phi_{n}(x) d x .
\end{aligned}
$$

Setting $\tilde{v}_{n}(x)=v_{n}\left(x+y_{n}\right)$ and $\tilde{u}_{n}(x)=u_{n}\left(x+y_{n}\right)$, note that $\left(\tilde{v}_{n}\right)$ is bounded in $E$. In fact, $\left(v_{n}\right)$ is bounded and by the invariance of $H^{1}\left(\mathbb{R}^{N}\right)$-norm due to translations, it follows that

$$
\left\|\tilde{v}_{n}\right\|_{E} \leq c_{1}\left\|\tilde{v}_{n}\right\|_{H^{1}\left(\mathbb{R}^{N}\right)}=c_{1}\left\|v_{n}\right\|_{H^{1}\left(\mathbb{R}^{N}\right)} \leq c_{2}\left\|v_{n}\right\|_{E}=c_{2} .
$$

Hence, up to subsequences,

$$
\begin{array}{rlll}
\tilde{v}_{n} \rightarrow \tilde{v}=\tilde{v}^{+}+\tilde{v}^{-}+\tilde{v}^{0} & \text { in } & E=E^{+}+E^{-}+E^{0}, \\
\tilde{v}_{n} \rightarrow \tilde{v} & \text { in } & L_{l o c}^{2}\left(\mathbb{R}^{N}\right) .
\end{array}
$$


Then, making a change of variables, it yields

$$
\begin{aligned}
o_{n}(1) & =\frac{1}{\left\|u_{n}\right\|} I^{\prime}\left(u_{n}\right) \phi_{n} \\
& =\int_{\mathbb{R}^{N}}\left(\nabla v_{+, n}(x) \nabla \phi\left(x-y_{n}\right)+v_{+, n}(x) V(x) \phi\left(x-y_{n}\right)\right) d x \\
& +\int_{\mathbb{R}^{N}}\left(\nabla v_{-, n}(x) \nabla \phi\left(x-y_{n}\right)+v_{-, n}(x) V(x) \phi\left(x-y_{n}\right)\right) d x \\
& -\int_{\mathbb{R}^{N}} h(x) \frac{f\left(u_{n}(x)\right)}{u_{n}(x)} v_{n}(x) \phi\left(x-y_{n}\right) d x \\
& =\int_{\mathbb{R}^{N}}\left(\nabla \tilde{v}_{+, n}(x) \nabla \phi(x)+\tilde{v}_{+, n}(x) V\left(x+y_{n}\right) \phi(x)\right) d x \\
& +\int_{\mathbb{R}^{N}}\left(\nabla \tilde{v}_{-, n}(x) \nabla \phi(x)+\tilde{v}_{-, n}(x) V\left(x+y_{n}\right) \phi(x)\right) d x \\
& -\int_{\mathbb{R}^{N}} h\left(x+y_{n}\right) \frac{f\left(\tilde{u}_{n}(x)\right)}{\tilde{u}_{n}(x)} \tilde{v}_{n}(x) \phi(x) d x .
\end{aligned}
$$

Now consider two cases:

Case 1. $\left|y_{n}\right| \rightarrow+\infty$. Let $K=\operatorname{supp}(\phi)$ be the support of $\phi$. Note that due to Remark 2.6 $|f(s)| \leq \kappa|s|$ for all $s \in \mathbb{R}$, and from 2.38) there exists $\zeta \in L^{1}(K)$ such that $\left|\tilde{v}_{n}(x)\right| \leq \zeta(x)$, almost everywhere in $K$. Thus,

$$
\left|h\left(\cdot+y_{n}\right) \frac{f\left(\tilde{u}_{n}(\cdot)\right)}{\tilde{u}_{n}(\cdot)} \tilde{v}_{n}(\cdot) \phi(\cdot)\right| \leq h_{\infty} \kappa \zeta(\cdot) \phi(\cdot) \in L^{1}(K)
$$

On the other hand, $\tilde{v}_{n}(x) \rightarrow \tilde{v}(x)$, almost everywhere in $K$, and $h(x) \rightarrow 0$ as $|x| \rightarrow+\infty$, in virtue of $\left(h_{1}\right)$, then

$$
\left|h\left(x+y_{n}\right) \frac{f\left(\tilde{u}_{n}(x)\right)}{\tilde{u}_{n}(x)} \tilde{v}_{n}(x) \phi(x)\right| \leq \kappa\left|\tilde{v}_{n}(x) \phi(x)\right|\left|h\left(x+y_{n}\right)\right| \rightarrow 0,
$$

almost everywhere in $K$ as $n \rightarrow+\infty$. Therefore from Lebesgue Dominated Convergence Theorem, it follows that

$$
\int_{\mathbb{R}^{N}} h\left(x+y_{n}\right) \frac{f\left(\tilde{u}_{n}(x)\right)}{\tilde{u}_{n}(x)} \tilde{v}_{n}(x) \phi(x) d x=\int_{K} h\left(x+y_{n}\right) \frac{f\left(\tilde{u}_{n}(x)\right)}{\tilde{u}_{n}(x)} \tilde{v}_{n}(x) \phi(x) d x \rightarrow 0,
$$

as $n \rightarrow+\infty$. Moreover, $V\left(x+y_{n}\right) \rightarrow V_{\infty}$ almost everywhere in $\mathbb{R}^{N}$ as $n \rightarrow+\infty$, in view of $\left(V_{1}\right)$. Thus, 2.39 and 2.40 yield

$$
\begin{aligned}
o_{n}(1) & =\int_{K}\left[\nabla \tilde{v}_{+, n}(x) \nabla \phi(x)+\left(V_{\infty}+o_{n}(1)\right) \tilde{v}_{+, n}(x) \phi(x)\right] d x \\
& +\int_{K}\left[\nabla \tilde{v}_{-, n}(x) \nabla \phi(x)+\left(V_{\infty}+o_{n}(1)\right) \tilde{v}_{-, n}(x) \phi(x)\right] d x
\end{aligned}
$$


Taking $n \rightarrow+\infty$, for any $\phi \in C_{0}^{\infty}\left(\mathbb{R}^{N}\right)$, it follows that

$$
\int_{\mathbb{R}^{N}}\left[\nabla\left(\tilde{v}_{+}(x)+\tilde{v}_{-}(x)\right) \nabla \phi+V_{\infty}\left(\tilde{v}_{+}(x)+\tilde{v}_{-}(x)\right) \phi(x)\right] d x=0,
$$

thus, $w=\tilde{v}^{+}+\tilde{v}^{-}$is a weak solution to problem $-\Delta w(x)+V_{\infty} w(x)=0$, in $\mathbb{R}^{N}$. Since operator Laplacian in $H^{1}\left(\mathbb{R}^{N}\right)$ does not have eigenfunctions, it implies that $w=0$. Thus, $\tilde{v}=\tilde{v}^{0} \in E^{0}$. If $E_{0}=\{0\}$, then $\tilde{v}=0$ and it yields a contradiction. In fact, by $(i i)$ and 2.38

$$
\int_{B_{r}(0)}|\tilde{v}(x)|^{2} d x=\limsup _{n \rightarrow+\infty} \int_{B_{r}(0)}\left|\tilde{v}_{n}(x)\right|^{2} d x=\limsup _{n \rightarrow+\infty} \int_{B_{r}\left(y_{n}\right)}\left|v_{n}(x)\right|^{2} d x>\varrho>0 .
$$

On the other hand, if $E^{0}$ is nontrivial, since $\left\|v_{0, n}\right\|=\left\|\tilde{v}_{0, n}\right\| \leq 1$ and $E^{0}$ is finite dimensional, it implies that $v_{0, n} \rightarrow v^{0}$ in $E$ up to subsequences, with $\left\|v^{0}\right\|=\left\|\tilde{v}^{0}\right\| \neq 0$. Hence

$$
\begin{aligned}
o_{n}(1) & =\frac{1}{\left\|u_{n}\right\|} I^{\prime}\left(u_{n}\right) v^{0} \\
& =\int_{\mathbb{R}^{N}}\left(\nabla v_{+, n}(x) \nabla v^{0}(x)+v_{+, n}(x) V(x) v^{0}(x)\right) d x \\
& +\int_{\mathbb{R}^{N}}\left(\nabla v_{-, n}(x) \nabla v^{0}(x)+v_{-, n}(x) V(x) v^{0}(x)\right) d x \\
& -\int_{\mathbb{R}^{N}} h(x) \frac{f\left(u_{n}(x)\right)}{u_{n}(x)} v_{n}(x) v^{0}(x) d x \\
& =-\int_{\mathbb{R}^{N}} h(x) \frac{f\left(u_{n}(x)\right)}{u_{n}(x)} v_{0, n}(x) v^{0}(x) d x .
\end{aligned}
$$

Since $h(x) \frac{f\left(u_{n}(x)\right)}{u_{n}(x)} v_{0, n}(x) v^{0}(x) \rightarrow a h(x)\left(v^{0}(x)\right)^{2}$, almost everywhere in $\operatorname{supp}\left(v^{0}\right)$, in virtue of the same arguments as above, by applying Lebesgue Dominated Convergence Theorem in 2.41) it yields

$$
\int_{\mathbb{R}^{N}} a h(x)\left(v^{0}(x)\right)^{2} d x=0,
$$

which contradicts $v^{0} \not \equiv 0$. Therefore $(i i)$ does not occur in this case.

Case 2. $\left(y_{n}\right)$ is bounded. Provided that

$$
\left\|\tilde{u}_{n}\right\| \geq \frac{c_{1}}{c_{2}}\left\|\tilde{u}_{n}\right\|_{H^{1}\left(\mathbb{R}^{N}\right)}=\frac{c_{1}}{c_{2}}\left\|u_{n}\right\|_{H^{1}\left(\mathbb{R}^{N}\right)} \geq \frac{1}{c_{2}}\left\|u_{n}\right\|,
$$

then $\left\|\tilde{u}_{n}\right\| \rightarrow+\infty$ as $n \rightarrow+\infty$. Moreover, there exists $\Omega \subset B_{r}(0)$, such that the Lebesgue measure is $\mu(\Omega)>0$ and

$$
0 \neq|\tilde{v}(x)|=\lim _{n \rightarrow+\infty}\left|\tilde{v}_{n}(x)\right|=\lim _{n \rightarrow+\infty} \frac{\left|\tilde{u}_{n}(x)\right|}{\left\|\tilde{u}_{n}\right\|}
$$


almost everywhere in $\Omega$, since $\tilde{v} \not \equiv 0$ in $B_{r}(0)$. Provided that $\left\|\tilde{u}_{n}\right\| \rightarrow+\infty$, it follows that $\left|\tilde{u}_{n}(x)\right| \rightarrow+\infty$, almost everywhere in $\Omega$. Thus, $\Omega \subset B_{r}(0)$ and $h>0$ in $\overline{B_{r}(0)}$ implies $0<h_{0}=\inf _{x \in \overline{B_{r}(0)}}\{h(x)\}$ and $\left(f_{3}\right)$ yields

$$
\begin{aligned}
& \liminf _{n \rightarrow+\infty} \int_{\mathbb{R}^{N}} h(x)\left[\frac{1}{2} f\left(u_{n}(x)\right) u_{n}(x)-F\left(u_{n}(x)\right)\right] d x \\
= & \liminf _{n \rightarrow+\infty} \int_{\mathbb{R}^{N}} h\left(x+y_{n}\right)\left[\frac{1}{2} f\left(\tilde{u}_{n}(x)\right) \tilde{u}_{n}(x)-F\left(\tilde{u}_{n}(x)\right)\right] d x \\
\geq & h_{0} \int_{\Omega} \liminf _{n \rightarrow+\infty}\left[\frac{1}{2} f\left(\tilde{u}_{n}(x)\right) \tilde{u}_{n}(x)-F\left(\tilde{u}_{n}(x)\right)\right] d x \\
= & +\infty,
\end{aligned}
$$

since $Q(s) \rightarrow+\infty$ as $|s| \rightarrow+\infty$. However, this is a contradiction with the fact that

$$
\int_{\mathbb{R}^{N}} h(x)\left[\frac{1}{2} f\left(u_{n}(x)\right) u_{n}(x)-F\left(u_{n}(x)\right)\right] d x=I\left(u_{n}\right)-\frac{1}{2} I^{\prime}\left(u_{n}\right) u_{n}=c+o_{n}(1) .
$$

Hence, $(i i)$ does not occur neither in Case 2. Therefore non-vanishing does not hold for $\left(v_{n}\right)$.

On the other hand, suppose that vanishing case holds for $\left(v_{n}\right)$, up to subsequences. Since $\left(u_{n}\right)$ is a Cerami sequence then $I^{\prime}\left(u_{n}\right) u_{+, n} \rightarrow 0$ and $I^{\prime}\left(u_{n}\right) u_{-, n} \rightarrow 0$ as $n \rightarrow+\infty$. Therefore,

$$
o_{n}(1)=\frac{I^{\prime}\left(u_{n}\right) u_{+, n}}{\left\|u_{n}\right\|^{2}}=\frac{1}{\left\|u_{n}\right\|} I^{\prime}\left(u_{n}\right) v_{+, n}=\left\|v_{+, n}\right\|^{2}-\int_{\Omega_{n}} h(x)\left[\frac{f\left(u_{n}(x)\right)}{u_{n}(x)} v_{n}(x) v_{+, n}(x)\right] d x
$$

and

$$
o_{n}(1)=\frac{I^{\prime}\left(u_{n}\right) u_{-, n}}{\left\|u_{n}\right\|^{2}}=\frac{1}{\left\|u_{n}\right\|} I^{\prime}\left(u_{n}\right) v_{-, n}=-\left\|v_{-, n}\right\|^{2}-\int_{\Omega_{n}} h(x)\left[\frac{f\left(u_{n}(x)\right)}{u_{n}(x)} v_{n}(x) v_{-, n}(x)\right] d x .
$$

Subtracting the latter equation from the former one it yields

$$
\begin{aligned}
o_{n}(1) & =\left\|v_{+, n}\right\|^{2}+\left\|v_{-, n}\right\|^{2}-\int_{\Omega_{n}} h(x)\left[\frac{f\left(u_{n}(x)\right)}{u_{n}(x)} v_{n}(x)\left(v_{+, n}(x)-v_{-, n}(x)\right)\right] d x \\
& =1-\left\|v_{0, n}\right\|^{2}-\int_{\Omega_{n}} h(x)\left[\frac{f\left(u_{n}(x)\right)}{u_{n}(x)}\left(v_{+, n}^{2}(x)-v_{-, n}^{2}(x)\right)\right] d x .
\end{aligned}
$$

Thus,

$$
\int_{\Omega_{n}} h(x)\left[\frac{f\left(u_{n}(x)\right)}{u_{n}(x)}\left(v_{+, n}^{2}(x)-v_{-, n}^{2}(x)\right)\right] d x \rightarrow 1-\left\|v^{0}\right\|^{2},
$$

as $n \rightarrow+\infty$. However, since $\left|\frac{f(s)}{s}\right| \leq \kappa$, for all $s \in \mathbb{R} \backslash\{0\}$ and provided that $h \in L^{q}\left(\mathbb{R}^{N}\right)$, with $q=\frac{2^{*}}{2^{*}-p}$ and $2<2 \frac{2^{*}}{p}<2^{*}$, Hölder's Inequality for $q$ and $q^{\prime}=\frac{2^{*}}{p}$ and Minkowski's Inequality yield 


$$
\begin{aligned}
\left|\int_{\Omega_{n}} h(x) \frac{f\left(u_{n}(x)\right)}{u_{n}(x)}\left(v_{+, n}^{2}(x)-v_{-, n}^{2}(x)\right) d x\right| & \leq \kappa\|h\|_{L^{q}\left(\mathbb{R}^{N}\right)}\left[\left\|v_{+, n}^{2}\right\|_{L^{\frac{2^{*}}{p}}\left(\mathbb{R}^{N}\right)}+\left\|v_{-, n}^{2}\right\|_{L^{2^{\frac{2}{p}}}\left(\mathbb{R}^{N}\right)}\right] \\
& =\kappa\|h\|_{L^{q}\left(\mathbb{R}^{N}\right)}\left[\left\|v_{+, n}\right\|_{L^{2^{\frac{2 *}{p}}}\left(\mathbb{R}^{N}\right)}^{2}+\left\|v_{-, n}\right\|_{L^{2 \frac{2^{*}}{p}}\left(\mathbb{R}^{N}\right)}^{2}\right] \\
& \rightarrow 0,
\end{aligned}
$$

as $n \rightarrow+\infty$, provided that $\left\|v_{j, n}\right\|_{L^{2 \frac{2^{*}}{p}\left(\mathbb{R}^{N}\right)}} \leq\left\|v_{n}\right\|_{L^{2 \frac{2^{*}}{p}}\left(\mathbb{R}^{N}\right)} \rightarrow 0$, for $j=+,-$, by Lions' Lemma (cf. [35] and also [57, Lemma 1.21 page 16). Hence, combining (2.42) and (2.43) it yields $\left\|v^{0}\right\|_{L^{2}\left(\mathbb{R}^{N}\right)}=\left\|v^{0}\right\|=1$ which also contradicts vanishing condition. Thus, it does not hold either. Therefore $(i)$ cannot occur for $\left(v_{n}\right)$ and this proof is complete.

\subsubsection{Setting Some Necessary Hypotheses}

Next step is to prove that $I$ satisfies $\left(I_{1}\right),\left(I_{2}\right),\left(I_{3}\right)$ and $\left(I_{4}\right)$ in Theorem 1.4 . Throughout this subsection, conditions $\left(I_{1}\right)$ and $\left(I_{2}\right)$ are proved. First of all, see that $I \in C^{1}(E, \mathbb{R})$, due to the hypotheses assumed about $h$ and $f$. Moreover, on one hand,

$$
(L u, u)=\left(L_{1} u_{1}+L_{2} u_{2}, u_{1}+u_{2}\right)=\left(L_{1} u_{1}, u_{1}\right)+\left(L_{2} u_{2}, u_{2}\right)
$$

and on the other hand, denoting by $I_{1}: E_{1} \rightarrow E_{1}$ the identity operator in $E_{1}$, and by $P^{-}: E_{2} \rightarrow E_{2}$ the projector operator of $E_{2}$ on $E^{-}$, note that $u_{2} \in E_{2}$ is such that $u_{2}=u^{-}+u^{0}$, hence

$$
\begin{aligned}
\left\|u^{+}\right\|^{2}-\left\|u^{-}\right\|^{2} & =\left\|u_{1}\right\|^{2}-\left\|u_{2}-u^{0}\right\|^{2} \\
& =\left(u_{1}, u_{1}\right)-\left(u_{2}-u^{0}, u_{2}-u^{0}\right) \\
& =\left(I_{1}\left(u_{1}\right), u_{1}\right)+\left(-P^{-}\left(u_{2}\right), u_{2}\right)
\end{aligned}
$$

Thus, setting $L_{1}:=I_{1}$ and $L_{2}:=-P^{-}$, it follows that $L_{i}: E_{i} \rightarrow E_{i}$ are bounded, linear and self-adjoint operators, for $i=1,2$. Therefore $I(u)=\frac{1}{2}(L u, u)+B(u)$, where

$$
B(u)=-\int_{\mathbb{R}^{N}} h(x) F(u(x)) d x
$$

and this gives $\left(I_{1}\right)$.

In order to prove $\left(I_{2}\right)$ the following lemma is needed.

Lemma 2.10. Assume that $\left(h_{1}\right)-\left(h_{2}\right)$ and $\left(f_{1}\right)-\left(f_{2}\right)$ hold for $I$, then $B$ given in (2.44) is weakly continuous and uniformly differentiable on bounded subsets.

Proof. Let $u_{n} \rightarrow u$ be a sequence in $E$, then $u_{n}(x) \rightarrow u(x)$ almost everywhere in $\mathbb{R}^{N}$, and $F\left(u_{n}\right)(x) \rightarrow F(u)(x)$ almost everywhere in $\mathbb{R}^{N}$, since $F(s)$ is a continuous function. Moreover, 
(2.36) yields

$$
\left|F\left(u_{n}\right)\right|^{\frac{2^{*}}{p}} \leq\left(\frac{\varepsilon}{2}\left|u_{n}\right|^{2}+\frac{C_{\varepsilon}}{p}\left|u_{n}\right|^{p}\right)^{\frac{2^{*}}{p}} \leq C\left(\frac{\varepsilon}{2}\left|u_{n}\right|^{2^{\frac{2^{*}}{p}}}+\frac{C_{\varepsilon}}{p}\left|u_{n}\right|^{2^{*}}\right) \in L^{1}\left(\mathbb{R}^{N}\right),
$$

since $2<2 \frac{2^{*}}{p}<2^{*}$ and $\left(u_{n}\right) \subset E \hookrightarrow L^{s}\left(\mathbb{R}^{N}\right)$, for $2 \leq s \leq 2^{*}$. Thus $\left(F\left(u_{n}(\cdot)\right)\right) \subset L^{\frac{2^{*}}{p}}\left(\mathbb{R}^{N}\right)$ is bounded, provided that $\left(u_{n}\right)$ is bounded in $E$ and then it is bounded in $L^{2 \frac{2^{*}}{p}}\left(\mathbb{R}^{N}\right)$ and in $L^{2^{*}}\left(\mathbb{R}^{N}\right)$. Since $F\left(u_{n}\right)(x) \rightarrow F(u)(x)$ almost everywhere in $\mathbb{R}^{N}$ and $\left\|F\left(u_{n}\right)\right\|_{L^{\frac{2^{*}}{p}\left(\mathbb{R}^{N}\right)}} \leq C$ for all $n \in \mathbb{N}$, by Brezis-Lieb's Lemma in [10], $F\left(u_{n}\right) \rightarrow F(u)$ in $L^{\frac{2^{*}}{p}}\left(\mathbb{R}^{N}\right)$. Provided that $\left(h_{2}\right)$ implies that $h \in L^{q}\left(\mathbb{R}^{N}\right)$, where $q$ is the conjugate exponent of $\frac{2^{*}}{p}$, then

$$
\int_{\mathbb{R}^{N}} h(x) F\left(u_{n}(x)\right) d x \rightarrow \int_{\mathbb{R}^{N}} h(x) F(u(x)) d x,
$$

as $n \rightarrow+\infty$. Therefore $B$ is weakly continuous.

Showing that $B$ is uniformly differentiable on bounded subsets of $E$ means that given $\varepsilon>0$ and $B_{R} \subset E$, there exists $\delta>0$ such that $\left|B(u+v)-B(u)-B^{\prime}(u) v\right|<\varepsilon\|v\|$, for all $u+v \in B_{R}$ with $\|v\|<\delta$. First, note that $B$ satisfies

$$
\begin{aligned}
\left|B(u+v)-B(u)-B^{\prime}(u) v\right| & =\left|\int_{\mathbb{R}^{N}} h(x)[F(u(x)+v(x))-F(u(x))-f(u(x)) v(x)] d x\right| \\
& \leq \int_{\mathbb{R}^{N}} h(x)|f(z(x))-f(u(x))||v(x)| d x \\
& \leq h_{\infty}^{\frac{1}{2}} \int_{\mathbb{R}^{N}}(h(x))^{\frac{1}{2}}|f(z(x))-f(u(x))||v(x)| d x
\end{aligned}
$$

since for $\psi(t):=F(u+t v)$, it yields $\psi^{\prime}(t)=f(u+t v) v$. Hence, Mean Value Theorem implies there exists some function $\theta(x)$, such that $0<\theta(x)<1$, almost everywhere in $\mathbb{R}^{N}$ and writing $z=u+\theta v$, it follows that

$$
F(u+v)-F(u)=\psi(1)-\psi(0)=\psi^{\prime}(\theta)=f(z) v,
$$

almost everywhere in $\mathbb{R}^{N}$. Moreover, provided that $h \in L^{\infty}\left(\mathbb{R}^{N}\right)$, from 2.45

$$
\begin{aligned}
\left|B(u+v)-B(u)-B^{\prime}(u) v\right| & \leq h_{\infty}^{\frac{1}{2}}\|\xi\|_{L^{2}\left(\mathbb{R}^{N}\right)}\|v\|_{L^{2}\left(\mathbb{R}^{N}\right)} \\
& \leq h_{\infty}^{\frac{1}{2}} C_{2}\|\xi\|_{L^{2}\left(\mathbb{R}^{N}\right)}\|v\|,
\end{aligned}
$$

where $C_{2}>0$ is the constant given by the continuous embedding $E \hookrightarrow L^{2}\left(\mathbb{R}^{N}\right)$ and $\xi:=h^{\frac{1}{2}}(\cdot)|f(z(\cdot))-f(u(\cdot))|$ it belongs to $L^{2}\left(\mathbb{R}^{N}\right)$. Indeed, since $\frac{2^{*}}{p}$ is the conjugate exponent of 
$q$, applying Hölder's Inequality for $q$ and $\frac{2^{*}}{p}$ it follows that

$$
\begin{aligned}
\int_{\mathbb{R}^{N}}|\xi|^{2} d x & =\int_{\mathbb{R}^{N}} h(x)|f(z(x))-f(u(x))|^{2} d x \\
& \leq\|h\|_{L^{q}\left(\mathbb{R}^{N}\right)}|| f(z(x))-f(u(x)) \|_{L^{2 \frac{2^{*}}{p}}\left(\mathbb{R}^{N}\right)}^{2} \\
& <+\infty
\end{aligned}
$$

since $2<2 \frac{2^{*}}{p}<2^{*}$ and $|f(u)|^{2 \frac{2^{*}}{p}} \leq \kappa^{2 \frac{2^{*}}{p}}|u|^{2 \frac{2^{*}}{p}} \in L^{1}\left(\mathbb{R}^{N}\right)$ due to Remark 2.6. Observe that by (2.46) is sufficient to show that given $\varepsilon>0$ and $B_{R} \subset E$, there exists $\delta>0$ such that $\|\xi\|_{L^{2}\left(\mathbb{R}^{N}\right)} \leq \frac{\varepsilon}{h_{\infty}^{\frac{1}{2}} C_{2}}$ for all $u+v \in B_{R}$ with $\|v\|<\delta$.

In order to prove this indirectly, suppose there exists $\varepsilon_{0}>0$ and $B_{R_{0}} \subset E$ fixed, such that for all $\delta>0$ it is possible to obtain $u_{\delta}+v_{\delta} \in B_{R_{0}}$ with $\left\|v_{\delta}\right\|<\delta$ and $\left\|\xi_{\delta}\right\|_{L^{2}\left(\mathbb{R}^{N}\right)}>\frac{\varepsilon_{0}}{h_{\infty}^{\frac{1}{2}} C_{2}}$, where

$$
\xi_{\delta}=h^{\frac{1}{2}}(\cdot)\left|f\left(z_{\delta}(\cdot)\right)-f\left(u_{\delta}(\cdot)\right)\right| \text { and } z_{\delta}=u_{\delta}+\theta v_{\delta} .
$$

Choosing $\delta_{n}=\frac{1}{n}$, for each $n \in \mathbb{N}$ there exist $u_{n}+v_{n} \in B_{R_{0}}$ such that $\left\|v_{n}\right\| \leq \frac{1}{n}$ and

$$
\left\|\xi_{n}\right\|_{L^{2}\left(\mathbb{R}^{N}\right)}>\frac{\varepsilon_{0}}{h_{\infty}^{\frac{1}{2}} C_{2}} .
$$

Hence, $v_{n} \rightarrow 0$ in $E$ and $u_{n} \rightarrow u$ in $E$, up to subsequences as $n \rightarrow+\infty$. In addition, $z_{n} \rightarrow u$ in $E$ and $z_{n}(x), u_{n}(x) \rightarrow u(x)$ almost everywhere in $\mathbb{R}^{N}$, up to subsequences. Thus,

$$
\left|f\left(z_{n}(x)\right)-f\left(u_{n}(x)\right)\right|^{2} \rightarrow 0,
$$

almost everywhere in $\mathbb{R}^{N}$ as $n \rightarrow+\infty$. Moreover, $\left(z_{n}\right)$ and $\left(u_{n}\right)$ are bounded in $E$ and the Sobolev embedding $L^{2 \frac{2^{*}}{p}}\left(\mathbb{R}^{N}\right) \hookrightarrow E$ holds, then

$$
\begin{aligned}
\left\|\left|f\left(z_{n}\right)-f\left(u_{n}\right)\right|^{2}\right\|_{L^{\frac{2^{*}}{p}}\left(\mathbb{R}^{N}\right)}^{\frac{2^{*}}{p}} & \leq C\left(\left\|f\left(z_{n}\right)\right\|_{L^{2 \frac{2^{*}}{p}}\left(\mathbb{R}^{N}\right)}^{\frac{2^{*}}{p}}+\left\|f\left(u_{n}\right)\right\|_{L^{2 \frac{2^{*}}{p}\left(\mathbb{R}^{N}\right)}}^{2^{\frac{2^{*}}{p}}}\right) \\
& \leq C \kappa^{2 \frac{2^{*}}{p}}\left(\left\|z_{n}\right\|_{L^{2 \frac{2^{*}}{p}}\left(\mathbb{R}^{N}\right)}^{2 \frac{2^{*}}{p}}+\left\|u_{n}\right\|_{L^{2 \frac{2^{*}}{p}}\left(\mathbb{R}^{N}\right)}^{2 \frac{2^{*}}{p}}\right) \\
& \leq C\left(C_{2 \frac{2^{*}}{p}} \kappa\right)^{2 \frac{2^{*}}{p}}\left(\left\|z_{n}\right\|^{2 \frac{2^{*}}{p}}+\left\|u_{n}\right\|^{2 \frac{2^{*}}{p}}\right) \\
& \leq 2 C\left(C_{2 \frac{2^{*}}{p}} \kappa R_{0}\right)^{2 \frac{2^{*}}{p}} .
\end{aligned}
$$

Therefore, the sequence $\left(\left|f\left(z_{n}\right)-f\left(u_{n}\right)\right|^{2}\right)$ is bounded in $L^{\frac{2^{*}}{p}}\left(\mathbb{R}^{N}\right)$. Applying Brezis-Lieb's Lemma again, it yields $\left|f\left(z_{n}\right)-f\left(u_{n}\right)\right|^{2} \rightarrow 0$ in $L^{\frac{2^{*}}{p}}\left(\mathbb{R}^{N}\right)$ as $n \rightarrow+\infty$. Since $h \in L^{q}\left(\mathbb{R}^{N}\right)$, which is the 
dual space of $L^{\frac{2^{*}}{p}}\left(\mathbb{R}^{N}\right)$, by weak convergence it yields

$$
\left\|\xi_{n}\right\|_{L^{2}\left(\mathbb{R}^{N}\right)}^{2}=\int_{\mathbb{R}^{N}} h(x)\left|f\left(z_{n}(x)\right)-f\left(u_{n}(x)\right)\right|^{2} d x \rightarrow 0,
$$

as $n \rightarrow+\infty$, which contradicts $\left\|\xi_{n}\right\|_{L^{2}\left(\mathbb{R}^{N}\right)}>\frac{\varepsilon_{0}}{h_{\infty}^{\frac{1}{2}} C_{2}}$ and completes the proof.

\subsubsection{Linking Geometry}

This subsection is devoted to obtain the linking geometry in $\left(I_{3}\right)$, to observe that $\left(I_{4}\right)$ comes from the boundedness in Subsection 2.3.2, and finally to prove Theorem 2.3 .

First, for the linking structure, choose $Q=\left\{r e: r \in\left[0, r_{1}\right]\right\} \oplus\left(E_{2} \cap B_{r_{2}}\right)$, and $S=\partial B_{\rho} \cap E_{1}$, where $0<\rho<r_{1}<r_{2}$ are constants and $e \in E_{1}$, $\|e\|=1$, must be a suitable vector. Then, observe that if $a$ as in $\left(f_{2}\right)$ is such that $a>a_{0}$, for $\varepsilon>0$ small enough and $a_{\varepsilon}:=a-\varepsilon$, it follows that $a>a_{\varepsilon}>a_{0}$, and by the definition of $a_{0}$ in (2.34), there exists some $e_{0} \in E_{1}$ such that

$$
a_{0} \int_{\mathbb{R}^{N}} h(x) e_{0}^{2}(x) d x \leq\left\|e_{0}\right\|^{2} \leq a_{\varepsilon} \int_{\mathbb{R}^{N}} h(x) e_{0}^{2}(x) d x
$$

Normalizing $e_{0}$ it follows that $e:=\frac{e_{0}}{\left\|e_{0}\right\|} \in E_{1}$ is such that

$$
1=\|e\|^{2}=\int_{\mathbb{R}^{N}}\left(|\nabla e(x)|^{2}+V(x) e^{2}(x)\right) d x \leq a_{\varepsilon} \int_{\mathbb{R}^{N}} h(x) e^{2}(x) d x .
$$

Therefore, choose such $e$ for the structure of $Q$. Furthermore, by Lemma $1.1 \quad S$ and $\partial Q$ "link" , where $\partial Q$ can be written as $\partial Q=Q_{1} \cap Q_{2} \cap Q_{3}$, with $Q_{1}=\{0\} \oplus\left(E_{2} \cap B_{r_{2}}\right)$, $Q_{2}=\left\{r e: r \in\left[0, r_{1}\right]\right\} \oplus\left(E_{2} \cap \partial B_{r_{2}}\right)$ and $Q_{3}=\left\{r_{1} e\right\} \oplus\left(E_{2} \cap B_{r_{2}}\right)$. The following lemma shows that $I$ satisfies $\left(I_{3}\right)(i)-(i i)$ in Theorem 1.4 for some $\alpha>0, \omega=0$, and arbitrary $v \in E_{2}$.

Lemma 2.11. Assume that $\left(V_{1}\right)-\left(V_{2}\right),\left(h_{1}\right)-\left(h_{2}\right)$ and $\left(f_{1}\right)-\left(f_{2}\right)$ hold for $I$. For $Q$ and $S$ as above, and for sufficiently large $r_{1}>0,\left.I\right|_{S} \geq \alpha>0$ and $\left.I\right|_{\partial Q} \leq 0$ hold, for some $\alpha>0$.

Proof. By definition, $S \subset E_{1}$, hence for all $u_{1} \in S$ it yields

$$
\begin{aligned}
I\left(u_{1}\right) & =\frac{1}{2}\left\|u_{1}\right\|^{2}-\int_{\mathbb{R}^{N}} h(x) F\left(u_{1}(x)\right) d x \\
& \geq \frac{1}{2} \rho^{2}-h_{\infty} \int_{\mathbb{R}^{N}}\left(\frac{\varepsilon}{2}\left|u_{1}(x)\right|^{2}+\frac{C_{\varepsilon}}{p}\left|u_{1}(x)\right|^{p}\right) d x \\
& \geq \frac{1}{2} \rho^{2}-h_{\infty}\left(\frac{\varepsilon}{2} C_{2}^{2}|| u_{1}\left\|^{2}+\frac{C_{\varepsilon}}{p} C_{p}^{p}|| u_{1}\right\|^{p}\right) \\
& =\rho^{2}\left(\frac{1}{2}\left(1-\varepsilon h_{\infty} C_{2}^{2}\right)-\frac{C_{\varepsilon}}{p} h_{\infty} C_{p}^{p} \rho^{p-2}\right) .
\end{aligned}
$$


Thus, if $\varepsilon, \rho$ are sufficiently small, $1>\varepsilon h_{\infty} C_{2}^{2}$ and also

$$
d_{1}:=\frac{1}{2}\left(1-\varepsilon h_{\infty} C_{2}^{2}\right)>\frac{C_{\varepsilon}}{p} h_{\infty} C_{p}^{p} \rho^{p-2}=: d_{2},
$$

therefore from 2.48 it holds $I\left(u_{1}\right) \geq \rho^{2}\left(d_{1}-d_{2}\right)=\alpha>0$.

Now, for the purpose of checking that $\left.I\right|_{\partial Q} \leq 0<\alpha$, consider the three cases as follows:

Case i. $u \in Q_{1} \subset E_{2}$, thus

$$
I(u)=-\frac{1}{2}\|u\|^{2}-\int_{\mathbb{R}^{N}} h(x) F(u(x)) d x \leq 0,
$$

since $h(x) F(u(x)) \geq 0$, for all $x \in \mathbb{R}^{N}$.

Case ii. $u \in Q_{2}$, thus $u=u_{1}+u_{2}$, where $u_{1}=r e$, with $0 \leq\left\|u_{1}\right\|=r \leq r_{1}$ and $\left\|u_{2}\right\|=r_{2}>r_{1}$, therefore

$$
I(u)=\frac{1}{2}\left(\left\|u_{1}\right\|^{2}-r_{2}^{2}\right)-\int_{\mathbb{R}^{N}} h(x) F(u(x)) d x \leq \frac{1}{2}\left(r_{1}^{2}-r_{2}^{2}\right)<0 .
$$

Case iii. $u \in Q_{3}$, thus $u=r_{1} e+u_{2}$, where $0 \leq\left\|u_{2}\right\| \leq r_{2}$. If $r_{1} \leq\left\|u_{2}\right\| \leq r_{2}$, then

$$
I(u)=\frac{1}{2}\left(r_{1}^{2}-\left\|u_{2}\right\|^{2}\right)-\int_{\mathbb{R}^{N}} h(x) F(u(x)) d x \leq \frac{1}{2}\left(r_{1}^{2}-r_{1}^{2}\right) \leq 0 .
$$

If $0 \leq\left\|u_{2}\right\|<r_{1}$, put $u_{2}=r_{1} v_{2}$, where $v_{2} \in B_{1} \cap E_{2}$. Thus,

$$
\begin{aligned}
I(u) & =\frac{1}{2} r_{1}^{2}\left(1-\left\|v_{2}\right\|^{2}\right)-\int_{\mathbb{R}^{N}} h(x) F(u(x)) d x \\
& \leq \frac{1}{2} r_{1}^{2}\left(1-\int_{\mathbb{R}^{N}} 2 h(x) \frac{F\left(r_{1}\left(e(x)+v_{2}(x)\right)\right)}{r_{1}^{2}} d x\right) .
\end{aligned}
$$

Now, observe that $\left|2 h(\cdot) \frac{F\left(r_{1}\left(e(\cdot)+v_{2}(\cdot)\right)\right)}{r_{1}^{2}}\right| \leq h_{\infty} \kappa\left|e(\cdot)+v_{2}(\cdot)\right|^{2} \in L^{1}\left(\mathbb{R}^{N}\right)$, for all $r_{1}>0$, since $e, v_{2} \in L^{2}\left(\mathbb{R}^{N}\right)$. Moreover, from $\left(f_{2}\right)$ it follows that

$$
2 h(x) \frac{F\left(r_{1}\left(e(x)+v_{2}(x)\right)\right)}{r_{1}^{2}} \rightarrow a h(x)\left(e(x)+v_{2}(x)\right)^{2},
$$

almost everywhere in $\mathbb{R}^{N}$ as $r_{1} \rightarrow+\infty$. Lebesgue Dominated Convergence Theorem yields

$$
\int_{\mathbb{R}^{N}} 2 h(x) \frac{F\left(r_{1}\left(e(x)+v_{2}(x)\right)\right)}{r_{1}^{2}} d x \rightarrow a \int_{\mathbb{R}^{N}} h(x)\left[e(x)+v_{2}(x)\right]^{2} d x,
$$

as $r_{1} \rightarrow+\infty$, for all $v_{2} \in B_{1} \cap E_{2}$ fixed. 
Claim. The limit

$$
\lim _{r_{1} \rightarrow+\infty} \int_{\mathbb{R}^{N}} 2 h(x) \frac{F\left(r_{1}\left(e(x)+v_{2}(x)\right)\right)}{r_{1}^{2}} d x=a \int_{\mathbb{R}^{N}} h(x)\left[e(x)+v_{2}(x)\right]^{2} d x,
$$

is uniform for $v_{2} \in B_{1} \cap E_{2}$.

Assume that claim postponing its proof, so as to conclude case iii). From the uniform convergence in $B_{1} \cap E_{2}$, for each $\varepsilon>0$ there exists $r_{0}>0$ such that, for all $r_{1} \geq r_{0}$

$$
\int_{\mathbb{R}^{N}}\left[a-2 \frac{F\left(r_{1}\left(e(x)+v_{2}(x)\right)\right)}{r_{1}^{2}\left(e(x)+v_{2}(x)\right)^{2}}\right] h(x)\left(e(x)+v_{2}(x)\right)^{2} d x<\varepsilon \int_{\mathbb{R}^{N}} h(x)\left(e(x)+v_{2}(x)\right)^{2} d x,
$$

for all $v_{2} \in B_{1} \cap E_{2}$. Thus,

$$
\frac{1}{2} r_{1}^{2}\left(1-\int_{\mathbb{R}^{N}} 2 h(x) \frac{F\left(r_{1}\left(e(x)+v_{2}(x)\right)\right)}{r_{1}^{2}} d x\right)<\frac{1}{2} r_{1}^{2}\left(1-a_{\varepsilon} \int_{\mathbb{R}^{N}} h(x)\left(e(x)+v_{2}(x)\right)^{2} d x\right),
$$

where $a_{\varepsilon}=(a-\varepsilon)$. Now, observe that $e$ and $v_{2}$ are orthogonal, then

$$
\int_{\mathbb{R}^{N}} h(x)\left(e(x)+v_{2}(x)\right)^{2} d x=\int_{\mathbb{R}^{N}} h(x)\left[e^{2}(x)+v_{2}^{2}(x)\right] d x \geq \int_{\mathbb{R}^{N}} h(x) e^{2}(x) d x .
$$

Hence, from 2.50)

$$
\frac{1}{2} r_{1}^{2}\left(1-\int_{\mathbb{R}^{N}} 2 h(x) \frac{F\left(r_{1}\left(e(x)+v_{2}(x)\right)\right)}{r_{1}^{2}} d x\right)<\frac{1}{2} r_{1}^{2}\left(1-a_{\varepsilon} \int_{\mathbb{R}^{N}} h(x) e^{2}(x) d x\right),
$$

and thus, substituting in 2.49 , it yields

$$
I(u)<\frac{1}{2} r_{1}^{2}\left(1-a_{\varepsilon} \int_{\mathbb{R}^{N}} h(x) e^{2}(x) d x\right) .
$$

Therefore, from (2.47) and (2.51) it follows that $I(u)<0$, and the result holds.

In order to prove the claim, it is necessary to prove that

$$
\lim _{r_{1} \rightarrow+\infty} \int_{\mathbb{R}^{N}}\left[a-2 \frac{F\left(r_{1}\left(e(x)+v_{2}(x)\right)\right)}{r_{1}^{2}\left(e(x)+v_{2}(x)\right)^{2}}\right] h(x)\left(e(x)+v_{2}(x)\right)^{2} d x=0,
$$

uniformly in $v_{2} \in B_{1} \cap E_{2}$, which gives the result. First of all, define for all $n \in \mathbb{N}$, functional $J_{n}: B_{1} \cap E_{2} \rightarrow \mathbb{R}$ given by

$$
J_{n}\left(v_{2}\right):=\int_{\mathbb{R}^{N}}\left[a-2 \frac{F\left(n\left(e(x)+v_{2}(x)\right)\right)}{n^{2}\left(e(x)+v_{2}(x)\right)^{2}}\right] h(x)\left(e(x)+v_{2}(x)\right)^{2} d x .
$$


The continuity of $F$ implies that $J_{n}$ is continuous for all $n \in \mathbb{N}$. From $\left(f_{2}\right)$ and by the equivalence of $H_{1}$ and $E$ norms,

$$
0 \leq J_{n}\left(v_{2}\right) \leq a h_{\infty}\left(\|e\|_{L^{2}\left(\mathbb{R}^{N}\right)}^{2}+\left\|v_{2}\right\|_{L^{2}\left(\mathbb{R}^{N}\right)}^{2}\right) \leq 2 C_{2}^{2} a h_{\infty},
$$

for all $v_{2} \in B_{1} \cap E_{2}$. Then, seeing that $E_{2}$ is finite dimensional, $B_{1} \cap E_{2}$ is compact, and since $J_{n}$ is continuous in $B_{1} \cap E_{2}$, it attains a maximum value, denoted by $u_{n} \in B_{1} \cap E_{2}$. Considering this sequence of maximums $\left(u_{n}\right)$, and provided that $\left\|u_{n}\right\| \leq 1$ for all $n \in \mathbb{N}$, the sequence is bounded. Again, provided that $E_{2}$ is finite dimensional such a sequence converges, up to subsequences, in $B_{1} \cap E_{2}$, namely, $u_{n} \rightarrow u$ in the $E$-norm. Moreover, for all $v_{2} \in B_{1} \cap E_{2}$, for all $n \in \mathbb{N}$, $0 \leq J_{n}\left(v_{2}\right) \leq J_{n}\left(u_{n}\right)$ holds, that is

$$
\begin{aligned}
0 & \leq \int_{\mathbb{R}^{N}}\left[a-2 \frac{F\left(n\left(e(x)+v_{2}(x)\right)\right)}{n^{2}\left(e(x)+v_{2}(x)\right)^{2}}\right] h(x)\left(e(x)+v_{2}(x)\right)^{2} d x \\
& \leq \int_{\mathbb{R}^{N}}\left[a-2 \frac{F\left(n\left(e(x)+u_{n}(x)\right)\right)}{n^{2}\left(e(x)+u_{n}(x)\right)^{2}}\right] h(x)\left(e(x)+u_{n}(x)\right)^{2} d x .
\end{aligned}
$$

Now, note that $u_{n}(x) \rightarrow u(x)$ almost everywhere in $\mathbb{R}^{N}$, then from $\left(f_{2}\right)$

$$
\left[a-2 \frac{F\left(n\left(e(x)+u_{n}(x)\right)\right)}{n^{2}\left(e(x)+u_{n}(x)\right)^{2}}\right] h(x)\left(e(x)+u_{n}(x)\right)^{2} \rightarrow 0,
$$

almost everywhere in $\mathbb{R}^{N}$ as $n \rightarrow+\infty$. More than this, since $u_{n} \rightarrow u$ in $E$, then $u_{n} \rightarrow u$ in $L^{2}\left(\mathbb{R}^{N}\right)$, then there exists $\psi \in L^{1}\left(\mathbb{R}^{N}\right)$ such that $\left|u_{n}(x)\right|^{2} \leq \psi(x)$ almost everywhere in $\mathbb{R}^{N}$, thus

$$
0 \leq\left[a-2 \frac{F\left(n\left(e(\cdot)+u_{n}(\cdot)\right)\right)}{n^{2}\left(e(\cdot)+u_{n}(\cdot)\right)^{2}}\right] h(\cdot)\left(e(\cdot)+u_{n}(\cdot)\right)^{2} \leq a h_{\infty}(e(\cdot)+\psi(\cdot))^{2} \in L^{1}\left(\mathbb{R}^{N}\right) .
$$

By Lebesgue Dominated Convergence Theorem, it follows

$$
\lim _{n \rightarrow+\infty} \int_{\mathbb{R}^{N}}\left[a-2 \frac{F\left(n\left(e(x)+u_{n}(x)\right)\right)}{n^{2}\left(e(x)+u_{n}(x)\right)^{2}}\right] h(x)\left(e(x)+u_{n}(x)\right)^{2} d x=0,
$$

Now, applying in $(2.52)$ the limit as $n \rightarrow+\infty$, Sandwich Theorem yields

$$
\lim _{n \rightarrow+\infty} \int_{\mathbb{R}^{N}}\left[a-2 \frac{F\left(n\left(e(x)+v_{2}(x)\right)\right)}{n^{2}\left(e(x)+v_{2}(x)\right)^{2}}\right] h(x)\left(e(x)+v_{2}(x)\right)^{2} d x=0,
$$

uniformly for all $v_{2} \in B_{1} \cap E_{2}$ and the claim is proved.

In order to verify $\left(I_{4}\right)$, fix $b>0$ and take $\left(u_{n}\right)$ such that $I\left(u_{n}\right) \subset[c-b, c+b]$ and $\left\|I^{\prime}\left(u_{n}\right)\right\|\left(1+\left\|u_{n}\right\|\right) \rightarrow 0$ as $n \rightarrow+\infty$. Suppose that $\left(u_{n}\right)$ is unbounded and take $\left(u_{n_{k}}\right) \subset\left(u_{n}\right)$ such that $\left\|u_{n_{k}}\right\| \rightarrow+\infty$ as $k \rightarrow+\infty$. Seeing that $I\left(u_{n_{k}}\right) \subset[c-b, c+b]$ is bounded in $\mathbb{R}$, it 
implies that $I\left(u_{n_{k}}\right) \rightarrow d$, up to subsequences. Then, $\left(u_{n_{k}}\right)$ is a Cerami sequence on level $d$, up to subsequences, hence $\left(u_{n_{k}}\right)$ is bounded up to subsequences, by Lemma 2.9. However, it yields a contradiction, since $\left\|u_{n_{k}}\right\| \rightarrow+\infty$ as $k \rightarrow+\infty$. Thus, $\left(u_{n}\right)$ is bounded.

Now, after all theses results, one is finally ready to prove Theorem 2.3

Proof of Theorem 2.3. In view of all assumptions $I$ satisfies $\left(I_{1}\right),\left(I_{2}\right),\left(I_{3}\right)$ and $\left(I_{4}\right)$ in Theorem 1.4 so it is possible to apply this theorem for $I$. Theorem 1.4 provides a $c \geq \alpha>\omega=0$, critical value of $I$. Therefore, there exists $u \in E$ such that $I(u)=c>0$ and $I^{\prime}(u)=0$, hence, $u \neq 0$, since $I(u)>0$. Provided that $I \in C^{1}(E, \mathbb{R})$, it follows that $u$ is a nontrivial weak solution to $(P)$ in $H^{1}\left(\mathbb{R}^{N}\right)$.

Remark 2.7. Note that problem $(P)$ in 2.31), has just been solved with conditions on potential $V$, which ensures a spectrum with negative and positive parts. Such conditions implied that the subspace $E^{-}$, corresponding to negative spectrum, was finite dimensional (cf. Remark 2.5)). Although the fact of $E_{2}=E^{-} \oplus E^{0}$ being finite dimensional was not necessary to apply Theorem 2.3. this information was used to obtain the linking geometry (cf. the proof of claim in Lemma 2.11). However, with minor changes it is possible to prove the linking geometry indirectly, without the assumption that $\operatorname{dim} E_{2}$ is finite.

\subsubsection{A Schrödinger Operator with Purely Absolutely Continuous Spectrum}

Since Theorem 1.4 does not require that any subspace in the linking decomposition needs to be finite dimensional, the main goal of this section is to work with the same problem, but assuming on $V$ conditions which gives both subspaces in the linking decomposition being infinite dimensional. Henceforth, consider problem $(P)$, but replacing condition $\left(V_{1}\right)$ on $V$ by

$\left(V_{1}^{\prime}\right) V \in C\left(\mathbb{R}^{N}, \mathbb{R}\right)$ is $(2 \pi)$-periodic in $x_{i}, i=1, \ldots, N$

and also assuming condition $\left(V_{2}\right)$. Assumptions on $h$ and $f$ are the same as before.

Remark 2.8. In view of $\left(V_{1}^{\prime}\right)$, potential $V$ is periodic and continuous, hence bounded. In addition, Spectral Theory asserts that operator A has pure absolutely continuous spectrum, which is bounded from below and consists of closed disjoint intervals (cf. [9] Section 3.5). Namely, $\sigma(A)=\sigma_{\text {ess }}(A)=\sigma_{a c}(A)=\cup\left[\alpha_{i}, \beta_{i}\right]$. In view of $\left(V_{2}\right)$, operator $A$ has nonempty negative and positive spectra, and 0 is in the gap between them. Indeed, if $0 \in \sigma(A)$ it would be an isolated point of the spectrum, which contradicts the fact that operator A has pure continuous spectrum. 
Moreover, since $\sigma(A)=\sigma_{\text {ess }}(A)$ negative and positive spectrum are both part of essential spectrum. Thus, if $\{\mathscr{E}(\lambda)\}$ is the spectral family of operator $A$, by Spectral Theory and essential spectrum definition ( $c f$. [9] and [44]), the subspaces associated to negative and positive spectrum, namely $\mathscr{E}(0)\left(H^{1}\left(\mathbb{R}^{N}\right)\right)$ and $(I-\mathscr{E}(0))\left(H^{1}\left(\mathbb{R}^{N}\right)\right)$ are both infinite dimensional.

Setting $E_{1}:=(I-\mathscr{E}(0))\left(H^{1}\left(\mathbb{R}^{N}\right)\right)$ and $E_{2}:=\mathscr{E}(0)\left(H^{1}\left(\mathbb{R}^{N}\right)\right)$, these subspaces are such that operator $A$ is positive definite in the former and negative definite in the latter. Indeed, by the spectral family definition (cf. [9], Theorem 1.1', page 394; see also [39] Chapter 3), for all $u_{1} \in E_{1}$ and for all $u_{2} \in E_{2}$ it yields

$$
\sigma^{+}|| u_{1} \|_{L^{2}\left(\mathbb{R}^{N}\right)}^{2} \leq \int_{\mathbb{R}^{N}}\left(\left|\nabla u_{1}(x)\right|^{2}+V(x) u_{1}^{2}(x)\right) d x
$$

and

$$
-\sigma^{-}|| u_{2} \|_{L^{2}\left(\mathbb{R}^{N}\right)}^{2} \leq-\int_{\mathbb{R}^{N}}\left(\left|\nabla u_{2}(x)\right|^{2}+V(x) u_{2}^{2}(x)\right) d x .
$$

Because of this, it is possible to proceed similarly as before and consider the norm induced by operator $A$ :

$$
\|u\|^{2}:=\left\{\begin{array}{cl}
\int_{\mathbb{R}^{N}}\left(|\nabla u|^{2}+V(x) u^{2}\right) d x=(A u, u)_{L^{2}\left(\mathbb{R}^{N}\right)}, & \text { if } u \in E_{1}, \\
-\int_{\mathbb{R}^{N}}\left(|\nabla u|^{2}+V(x) u^{2}\right) d x=-(A u, u)_{L^{2}\left(\mathbb{R}^{N}\right)}, & \text { if } u \in E_{2},
\end{array}\right.
$$

which is equivalent to the usual norm in $H^{1}\left(\mathbb{R}^{N}\right)$. Denote $E=\left(H^{1}\left(\mathbb{R}^{N}\right),\|\cdot\|\right)$, then $E=E_{1} \oplus E_{2}$, namely every $u \in E$ can be uniquely written as $u=u_{1}+u_{2}$, with $u_{i} \in E_{i}$ and

$$
\|u\|^{2}=\left\|u_{1}\right\|^{2}+\left\|u_{2}\right\|^{2}=\left(A u_{1}, u_{1}\right)_{L^{2}\left(\mathbb{R}^{N}\right)}-\left(A u_{2}, u_{2}\right)_{L^{2}\left(\mathbb{R}^{N}\right)} .
$$

Here all conclusions from Remark 2.6 also hold, and again functional $I: E \rightarrow \mathbb{R}$ associated to $(P)$ is written as

$$
I(u)=\frac{1}{2}\left(\left\|u_{1}\right\|^{2}-\left\|u_{2}\right\|^{2}\right)-\int_{\mathbb{R}^{N}} h(x) F(u(x)) d x,
$$

for all $u=u_{1}+u_{2} \in E$.

Now it is possible to state the second main result of this section.

Theorem 2.4. Let consider problem $(P)$ with $V$ satisfying $\left(V_{1}^{\prime}\right)-\left(V_{2}\right)$, h satisfying $\left(h_{1}\right)-\left(h_{2}\right)$ and $f$ satisfying $\left(f_{1}\right)-\left(f_{3}\right)$, with $a>a_{0}$. Then $(P)$ has a nontrivial weak solution $u \in H^{1}\left(\mathbb{R}^{N}\right)$.

Under the purpose of applying Theorem 1.4 to solve this problem, proceeding as before it is necessary to prove the boundedness of Cerami sequences, and then, to show that this problem satisfies all required assumptions of Abstract Linking Theorem. 
Lemma 2.12. Assume that $\left(V_{1}^{\prime}\right)-\left(V_{2}\right),\left(h_{1}\right)-\left(h_{2}\right)$ and $\left(f_{1}\right)-\left(f_{3}\right)$ hold for $I$. Let $\left(u_{n}\right) \subset E$ be a Cerami sequence of $I$ in an arbitrary level $c \in \mathbb{R}$, then $\left(u_{n}\right)$ is bounded.

Proof. Seeking a contradiction, argue as in Lemma 2.9, showing that neither vanishing nor nonvanishing can hold for $\left(v_{n}\right)=\frac{u_{n}}{\left\|u_{n}\right\|}$. In order to show that vanishing case is impossible, the proof is exactly the same, considering the case $E^{0}=\{0\}$, so it is omitted here. For non-vanishing case, it is possible to follow the same lines as Lemma 2.9 up to the analysis of cases 1 and 2. Namely, when $\left(y_{n}\right) \subset \mathbb{R}^{N}$ given by non-vanishing condition is bounded or unbounded. Here, just assume that $\left(y_{n}\right)$ is always bounded. In fact, if $\left(y_{n}\right)$ is unbounded, provided that $V$ is $(2 \pi)$-periodic in $x_{i}, i=1, \ldots, N,\left(y_{n}\right)$ can be replaced by another bounded sequence $\left(x_{n}\right) \subset \mathbb{R}^{N}$ such that $V\left(x+y_{n}\right)=V\left(x+x_{n}\right)$ for all $n \in \mathbb{N}$. Arguing likewise as in Case 2, non-vanishing cannot hold either. Therefore, it yields a contradiction and $\left(u_{n}\right)$ is bounded.

Furthermore, observe that as before $I \in C^{1}(E, \mathbb{R})$, due to the hypotheses assumed about $h$ and $f$. Moreover, on one hand,

$$
(L u, u)=\left(L_{1} u_{1}+L_{2} u_{2}, u_{1}+u_{2}\right)=\left(L_{1} u_{1}, u_{1}\right)+\left(L_{2} u_{2}, u_{2}\right)
$$

and on the other hand, denoting by $I_{i}: E_{i} \rightarrow E_{i}$ the identity operator in $E_{i}$ for $i=1,2$, note that

$$
\left\|u_{1}\right\|^{2}-\left\|u_{2}\right\|^{2}=\left(u_{1}, u_{1}\right)-\left(u_{2}, u_{2}\right)=\left(I_{1}\left(u_{1}\right), u_{1}\right)+\left(-I_{2}\left(u_{2}\right), u_{2}\right) .
$$

Thus, setting $L_{i}:=(-1)^{i+1} I_{i}$ for $i=1,2$, it follows that $L_{i}: E_{i} \rightarrow E_{i}$ are bounded, linear and self-adjoint operators, for $i=1,2$. Thus, $I$ satisfies $\left(I_{1}\right)$ in Theorem 1.4 as before, and since all assumptions are kept on $h$ and $f$, then $\left(I_{2}\right)$ and $\left(I_{4}\right)$ also hold here with the same proofs. Therefore, it is only necessary to show the linking geometry in $\left(I_{3}\right)$, which will have a different proof in this case, provided that $E_{2}$ is infinite dimensional. First of all, set $S:=\partial B_{\rho} \cap E_{1}$ and $Q=\left\{r e+u_{2}: r \geq 0, u_{2} \in E_{2},\left\|r e+u_{2}\right\| \leq r_{1}\right\}$, where $0<\rho<r_{1}$ are constants and $e \in$ $E_{1},\|e\|=1$, is chosen as before. In fact, assuming $a>a_{0}$, by the definition of $a_{0}$, there exists some unitary $e \in E_{1}$ such that

$$
a_{0} \int_{\mathbb{R}^{N}} h(x) e^{2}(x) d x \leq\|e\|^{2}=1<a \int_{\mathbb{R}^{N}} h(x) e^{2}(x) d x .
$$

Such $e$ makes the following lemma true. Moreover, it is possible to show that $S$ and $Q$ "link" following the same lines as in Lemma 1.1. Next lemma shows the linking geometry $\left(I_{3}\right)(i)-(i i)$ of Theorem 1.4. for some $\alpha>0, \omega=0$ and arbitrary $v \in E_{2}$.

Lemma 2.13. Assume that $\left(V_{1}^{\prime}\right)-\left(V_{2}\right),\left(h_{1}\right)-\left(h_{2}\right)$ and $\left(f_{1}\right)-\left(f_{2}\right)$ hold for $I$. For $Q$ and $S$ as above, and for sufficiently large $r_{1}>0$, it follows that $\left.I\right|_{S} \geq \alpha>0$ and $\left.I\right|_{\partial Q} \leq 0$, for some $\alpha>0$. 
Proof. The proof that $\left.I\right|_{S} \geq \alpha>0$, for some $\alpha>0$, is the same in Lemma 2.11, thus it is not repeated here. In purpose of proving that $\left.I\right|_{\partial Q} \leq 0$, observe that $I\left(u_{2}\right) \leq 0$, for all $u_{2} \in E_{2}$, then it suffices to show that $I(r e+u) \leq 0$ for $r>0, u \in E_{2}$ and $\|r e+u\| \geq r_{1}$, for some $r_{1}>0$ large enough. Arguing indirectly assume that for some sequence $\left(r_{n} e+u_{n}\right) \subset \mathbb{R}^{+} e \oplus E_{2}$ such that $\left\|r_{n} e+u_{n}\right\| \rightarrow+\infty, I\left(r_{n} e+u_{n}\right)>0$ holds for all $n \in \mathbb{N}$. Then, seeking a contradiction, the desired result holds. Firstly, set $v_{n}:=\frac{r_{n} e+u_{n}}{\left\|r_{n} e+u_{n}\right\|}=s_{n} e+w_{n}$, where $s_{n} \in \mathbb{R}^{+}, w_{n} \in E_{2}$ and $\left\|v_{n}\right\|=1$. Provided that $\left(v_{n}\right)$ is bounded, up to subsequences it follows that $v_{n} \rightarrow v=s e+w$ in $E$. Then, $v_{n}(x) \rightarrow v(x)$ almost everywhere in $\mathbb{R}^{N}$, and seeing that $1=\left\|s_{n} e+w_{n}\right\|^{2}=s_{n}^{2}+\left\|w_{n}\right\|^{2}$, it ensures that $0 \leq s_{n}^{2} \leq 1, w_{n} \rightarrow w$ in $E$ and $s_{n} \rightarrow s$ in $\mathbb{R}^{+}$. Then it yields

$$
\begin{aligned}
\frac{I\left(r_{n} e+u_{n}\right)}{\left\|r_{n} e+u_{n}\right\|^{2}} & =\frac{s_{n}^{2}}{2}\|e\|^{2}-\frac{1}{2}\left\|w_{n}\right\|^{2}-\int_{\mathbb{R}^{N}} h(x) \frac{F\left(r_{n} e(x)+u_{n}(x)\right)}{\left\|r_{n} e+u_{n}\right\|^{2}} d x \\
& =s_{n}^{2}-\frac{1}{2}-\int_{\mathbb{R}^{N}} h(x) \frac{F\left(r_{n} e(x)+u_{n}(x)\right)}{\left\|r_{n} e+u_{n}\right\|^{2}} d x>0,
\end{aligned}
$$

and hence $\frac{1}{2}<s \leq 1$. Moreover, from 2.53 there exists a bounded domain $\Omega_{0} \subset \mathbb{R}^{N}$ such that

$$
1<a \int_{\Omega_{0}} h(x) e^{2}(x) d x
$$

Hence, $\operatorname{supp}(e) \cap \Omega_{0} \neq \emptyset$, and it follows that

$$
\begin{aligned}
0 & >s^{2}-s^{2} a \int_{\Omega_{0}} h(x) e^{2}(x) d x \\
& \geq s^{2}\left(1-a \int_{\Omega_{0}} h(x) e^{2}(x) d x\right)-\left(1-s^{2}\right)-a \int_{\Omega_{0}} h(x) w^{2}(x) d x \\
& =s^{2}\left(2-a \int_{\Omega_{0}} h(x) e^{2}(x) d x\right)-1-a \int_{\Omega_{0}} h(x) w^{2}(x) d x .
\end{aligned}
$$

On the other hand, since $v_{n} \rightarrow v$ in $E$, it converges strongly in $L^{2}\left(\Omega_{0}\right)$, then from assumption $\left(f_{1}\right)$ it follows that

$$
\left|h(\cdot) \frac{F\left(r_{n} e(\cdot)+u_{n}(\cdot)\right)}{\left\|r_{n} e+u_{n}\right\|^{2}}\right| \leq h_{\infty} \frac{\kappa}{2} v_{n}^{2}(\cdot) \leq \psi(\cdot) \in L^{1}\left(\Omega_{0}\right)
$$

In addition, by $\left(f_{2}\right)$, and since $\left\|r_{n} e+u_{n}\right\| \rightarrow+\infty$ as $n \rightarrow+\infty$, it also follows that

$$
h(x) \frac{F\left(r_{n} e(x)+u_{n}(x)\right)}{\left\|r_{n} e+u_{n}\right\|^{2}}=h(x) \frac{F\left(v_{n}(x)\left\|r_{n} e(x)+u_{n}(x)\right\|\right) v_{n}^{2}(x)}{v_{n}(x)^{2}\left\|r_{n} e+u_{n}\right\|^{2}} \rightarrow h(x) \frac{a}{2} v^{2}(x),
$$

almost everywhere in $\Omega_{0} \cap \operatorname{supp}(v)$, which is not empty since $v=s e+w,(e, w)_{L^{2}\left(\mathbb{R}^{N}\right)}=0$ and $\operatorname{supp}(e) \cap \Omega_{0} \neq \emptyset$. Thus, by Lebesgue Dominated Convergence Theorem, 


$$
\int_{\Omega_{0}} h(x) \frac{F\left(r_{n} e(x)+u_{n}(x)\right)}{\left\|r_{n} e+u_{n}\right\|^{2}} d x \rightarrow \frac{a}{2} \int_{\Omega_{0}} h(x)\left(s^{2} e^{2}(x)+w^{2}(x)\right) d x,
$$

as $n \rightarrow+\infty$. From (2.54)

$$
0<2 s_{n}^{2}-1-2 \int_{\Omega_{0}} h(x) \frac{F\left(r_{n} e(x)+u_{n}(x)\right)}{\left\|r_{n} e+u_{n}\right\|^{2}} d x
$$

and passing 2.54 to the limit as $n \rightarrow+\infty$, it yields

$$
\begin{aligned}
0 & \leq 2 s^{2}-1-a \int_{\Omega_{0}} h(x)\left(s^{2} e^{2}(x)+w^{2}(x)\right) d x \\
& =s^{2}\left(2-a \int_{\Omega_{0}} h(x) e^{2}(x) d x\right)-1-a \int_{\Omega_{0}} h(x) w^{2}(x) d x,
\end{aligned}
$$

which is contrary to 2.55. Therefore the lemma is proved.

By Lemma 2.13, functional $I$ satisfies $\left(I_{3}\right)$ of Theorem 1.4 . Now, Theorem 2.4 can be proved.

Proof of Theorem 2.4. As proved along this section, due to all hypotheses on $I$, it satisfies $\left(I_{1}\right)-$ $\left(I_{4}\right)$ in Theorem 1.4 Applying Theorem 1.4 it is obtained $c \geq \alpha>0$, a critical value of $I$. Thus, there exists $u \in E$, such that $I(u)=c>0$ and $I^{\prime}(u)=0$, provided that $I(u)>0$, then $u \neq 0$. Since $I \in C^{1}(E, \mathbb{R}), u$ is a nontrivial weak solution to $(P)$ in $H^{1}\left(\mathbb{R}^{N}\right)$. 


\section{Chapter}

\section{A Radial Nonlinear Schrödinger Equation}

This chapter aims to find solution to a radial nonlinear Schrödinger equation with a sign-changing potential and an asymptotically linear nonlinearity.

For $N \geq 3$ consider the radial problem $\left(P_{r}\right)$ given by

$$
-\Delta u+V(x) u=g(x, u) \text { in } \mathbb{R}^{N}
$$

where potential $V$ satisfies:

$\left(V_{1}\right)_{r} \quad V \in L^{\infty}\left(\mathbb{R}^{N}\right)$ is a radial sign-changing function, $V(x)=V(|x|)=V(r), r \geq 0 ;$

$\left(V_{2}\right)_{r}$ Setting $\bar{V}(r)=V(r)+\frac{(N-1)(N-3)}{4 r^{2}}$ and $\bar{A}:=-\frac{d^{2}}{d r^{2}}+\bar{V}(r)$, an operator of $L^{2}(0,+\infty)$, $0 \notin \sigma_{\text {ess }}(\bar{A})$ and

$$
\sup [\sigma(\bar{A}) \cap(-\infty, 0)]=\sigma^{-}<0<\sigma^{+}=\inf [\sigma(\bar{A}) \cap(0,+\infty)]
$$

Setting $A:=-\Delta+V(x)$ as an operator of $L^{2}\left(\mathbb{R}^{N}\right)$, since $V \in L^{\infty}\left(\mathbb{R}^{N}\right), A$ as well as $\bar{A}$ are self-adjoint operators. Due to Hardy's Inequality, operator $\bar{A}$ is treated in $H_{0}^{1}(0,+\infty)$, which can be written as $H_{0}^{1}(0,+\infty)=H^{-} \oplus H^{0} \oplus H^{+}$, with $H^{-}, H^{0}, H^{+}$the subspaces of $H_{0}^{1}(0,+\infty)$ where $\bar{A}$ is respectively negative, null and positive definite. In view of $\left(V_{2}\right)_{r}$ each $u \in H^{+}$satisfies $\sigma^{+}\|u\|_{L^{2}(0,+\infty)}^{2} \leq(\bar{A} u, u)_{L^{2}(0,+\infty)}$. Moreover, given $u \in H_{0}^{1}(0,+\infty)$ and setting $w:=r^{\frac{1-N}{2}} u$, it yields $w \in H_{\text {rad }}^{1}\left(\mathbb{R}^{N}\right)$ (cf. 53] Section 3), where $H_{r a d}^{1}\left(\mathbb{R}^{N}\right)$ is the subspace of the radially symmetric functions in $H^{1}\left(\mathbb{R}^{N}\right)$. In addition, changing variables, $w$ satisfies

$$
\|w\|_{L^{2}\left(\mathbb{R}^{N}\right)}^{2}=\int_{\mathbb{R}^{N}}|w(x)|^{2} d x=\omega_{N} \int_{0}^{+\infty}|u(x)|^{2} d r=\omega_{N}\|u\|_{L^{2}(0,+\infty)}^{2},
$$


and

$$
\begin{aligned}
(A w, w)_{L^{2}\left(\mathbb{R}^{N}\right)} & =\int_{\mathbb{R}^{N}}\left(|\nabla w(x)|^{2}+V(x) w(x)^{2}\right) d x \\
& =\omega_{N} \int_{0}^{+\infty}\left(\left|u^{\prime}(r)\right|^{2}+\bar{V}(r) u(r)\right) d r \\
& =\omega_{N}(\bar{A} u, u)_{L^{2}(0,+\infty)},
\end{aligned}
$$

where $\omega_{N}$ is the $(N-1)$-dimensional surface measure of the sphere $S^{N-1} \subset \mathbb{R}^{N}$. Hence $\sigma^{+}\|w\|_{L^{2}\left(\mathbb{R}^{N}\right)}^{2} \leq(A w, w)_{L^{2}\left(\mathbb{R}^{N}\right)}$. On the other hand, if some function $\tilde{w} \in H_{\text {rad }}^{1}\left(\mathbb{R}^{N}\right)$ satisfied

$$
0<(A \tilde{w}, \tilde{w})_{L^{2}\left(\mathbb{R}^{N}\right)}<\sigma^{+}\|\tilde{w}\|_{L^{2}\left(\mathbb{R}^{N}\right)}^{2}
$$

by density it could be regarded as a smooth function and then setting $\tilde{u}:=r^{\frac{N-1}{2}} \tilde{w}$, it would belong to $H^{+}$and would satisfy

$$
\sigma^{+}\|\tilde{u}\|_{L^{2}(0,+\infty)}^{2}>(\bar{A} \tilde{u}, \tilde{u})_{L^{2}(0,+\infty)}
$$

which contradicts $\left(V_{2}\right)_{r}$. Hence, writing $H_{r a d}^{1}\left(\mathbb{R}^{N}\right)=E^{-} \oplus E^{0} \oplus E^{+}$, with $E^{-}, E^{0}, E^{+}$the subspaces where $A$ is respectively negative, null and positive definite, if $w \in E^{+}$it satisfies $\sigma^{+}\|w\|_{L^{2}\left(\mathbb{R}^{N}\right)}^{2} \leq(A w, w)_{L^{2}\left(\mathbb{R}^{N}\right)}$.

Remark 3.1. Note that if $\sigma^{+}$is an eigenvalue of $\bar{A}$ with eigenfunction $u$, the same argument as above shows that $\sigma^{+}$is an eigenvalue of $A$, with a radial eigenfunction $w=r^{\frac{1-N}{2}} u \in E^{+}$. On the other hand, if $\sigma^{+}$is not an eigenvalue of $\bar{A}$, since it belongs to $\sigma_{\text {ess }}(\bar{A})$, given $\varepsilon>0$ there exist $u_{\varepsilon} \in H^{+}$such that

$$
\sigma^{+}\left\|u_{\varepsilon}\right\|_{L^{2}(0,+\infty)}^{2}<\left(\bar{A} u_{\varepsilon}, u_{\varepsilon}\right)_{L^{2}(0,+\infty)}<\left(\sigma^{+}+\varepsilon\right)\left\|u_{\varepsilon}\right\|_{L^{2}(0,+\infty)}^{2},
$$

which ensures that $w_{\varepsilon}:=r^{\frac{1-N}{2}} u_{\varepsilon} \in E^{+}$satisfies

$$
\sigma^{+}\left\|w_{\varepsilon}\right\|_{L^{2}\left(\mathbb{R}^{N}\right)}^{2}<\left(A w_{\varepsilon}, w_{\varepsilon}\right)_{L^{2}\left(\mathbb{R}^{N}\right)}<\left(\sigma^{+}+\varepsilon\right)\left\|w_{\varepsilon}\right\|_{L^{2}\left(\mathbb{R}^{N}\right)}^{2} .
$$

Therefore,

$$
\sigma^{+}=\inf _{w \in E^{+}} \frac{(A w, w)_{L^{2}\left(\mathbb{R}^{N}\right)}}{\|w\|_{L^{2}\left(\mathbb{R}^{N}\right)}^{2}} .
$$

Applying the same arguments comparing $H^{-}$and $E^{-}$, it yields

$$
-\sigma^{-}=\inf _{w \in E^{-}} \frac{-(A w, w)_{L^{2}\left(\mathbb{R}^{N}\right)}}{\|w\|_{L^{2}\left(\mathbb{R}^{N}\right)}^{2} .}
$$

From hypothesis $\left(V_{2}\right)_{r}$ either $0 \notin \sigma(\bar{A})$ or it is an isolated eigenvalue of $\bar{A}$. Since by assumption $0 \notin \sigma_{\text {ess }}(\bar{A})$, if $0 \in \sigma(\bar{A})$ it is an eigenvalue of finite multiplicity, hence $\operatorname{ker}(\bar{A})$ is 
finite dimensional. The same conclusions hold for $A$, since there exists a correspondence between the eigenfunctions of $\bar{A}$ and the radial eigenfunctions of $A$. Furthermore, $u_{1}, u_{2} \in H_{0}^{1}(0,+\infty)$ are orthogonal in $L^{2}(0,+\infty)$ iff $w_{1}=r^{\frac{1-N}{2}} u_{1}$ and $w_{2}=r^{\frac{1-N}{2}} u_{2}$ are orthogonal in $L^{2}\left(\mathbb{R}^{N}\right)$. Indeed,

$$
\int_{0}^{+\infty} u_{1}(r) u_{2}(r) d r=\frac{1}{\omega_{N}} \int_{\mathbb{R}^{N}} w_{1}(x) w_{2}(x) d x .
$$

Therefore, $H^{i}$ is infinite dimensional iff $E^{i}$ is infinite dimensional, for $i=-, 0,+$.

A typical example of $V$ satisfying $\left(V_{1}\right)_{r}-\left(V_{2}\right)_{r}$ is a suitable continuous, periodic and sign-changing $V(r)$, such that $0 \notin \sigma\left(-\frac{d^{2}}{d r^{2}}+V(r)\right)$, hence 0 is in a gap of the spectrum, which is composed by closed intervals. Since $V(r)$ is continuous and changes sign, $-\frac{d^{2}}{d r^{2}}+V(r)$ has positive and negative spectrum. Moreover, $\bar{V}=V+V_{N}$, where $V_{N}(r)=\frac{(N-1)(N-3)}{4 r^{2}}$ decays sufficiently fast, then it is a Kato's potential and hence $\bar{A}$-compact, which ensures $\sigma_{\text {ess }}(\bar{A})=\sigma\left(-\frac{d^{2}}{d r^{2}}+V(r)\right)$ by Weyl's theorem (cf. [37], Corollary 11.3.6 page 290, or 44] Corollary 2 page 113, and also [26] Sections 14.2-14.3), thus $0 \notin \sigma_{\text {ess }}(\bar{A})$ and $\sigma(\bar{A})$ also has positive and negative part. Therefore, $\left(V_{2}\right)_{r}$ is satisfied.

Remark 3.2. Simple examples of potentials which satisfy or not our assumptions:

Ex 1. $V(r)=\cos (r)$ satisfies $\left(V_{1}\right)_{r}-\left(V_{2}\right)_{r}$ by the previous observations.

Ex 2. $V(r)=\frac{1}{1+r^{2}}-\frac{1}{2}$, does not satisfy $\left(V_{2}\right)_{r}$, since $0 \in \sigma_{\text {ess }}(\bar{A})$. In fact, $\lim _{r \rightarrow+\infty} V(r)=-\frac{1}{2}$, hence $\sigma_{\text {ess }}(\bar{A})=\sigma_{\text {ess }}(A)=\left[-\frac{1}{2},+\infty\right)$.

Moreover, the nonlinearity $g$ satisfies:

$\left(g_{1}\right) g(x, s) \in C\left(\mathbb{R}^{N} \times \mathbb{R}, \mathbb{R}\right)$ is a radial function such that $\lim _{|s| \rightarrow 0} \frac{g(x, s)}{s}=0$, uniformly in $x$ and for all $t \in \mathbb{R}$,

$$
G(x, t)=\int_{0}^{t} g(x, s) d s \geq 0
$$

$\left(g_{2}\right) \lim _{|s| \rightarrow+\infty} \frac{g(x, s)}{s}=h(x)$, uniformly in $x$, where $h \in L^{\infty}\left(\mathbb{R}^{N}\right)$;

$\left(g_{3}\right) \quad a_{0}=\inf _{x \in \mathbb{R}^{N}} h(x)>\sigma^{+}=\inf [\sigma(A) \cap(0,+\infty)] ;$

$\left(g_{4}\right)$ Setting $\mathscr{O}:=A-\mathscr{H}$, where $\mathscr{H}$ is the operator multiplication by $h(x)$ in $L^{2}\left(\mathbb{R}^{N}\right)$ and denoting by $\sigma_{p}(\mathscr{O})$ the point spectrum of $\mathscr{O}$,

$$
0 \notin \sigma_{p}(\mathscr{O})
$$

$\left(g_{5}\right)$ Defining $Q(x, s):=\frac{1}{2} g(x, s) s-G(x, s) \geq 0$ for all $(x, s) \in \mathbb{R}^{N} \times \mathbb{R}$ and $\Sigma:=\min \left\{\sigma^{+},-\sigma^{-}\right\}$, 
there exists $\delta_{0}>0$ such that

$$
\frac{g(x, s)}{s} \geq \Sigma-\delta_{0} \Longrightarrow Q(x, s) \geq \delta_{0} .
$$

An example of $g$ satisfying $\left(g_{1}\right)-\left(g_{5}\right)$ is an asymptotically linear continuous function such that $h(x) \equiv a_{0}>\sigma^{+}$as in $\left(g_{3}\right)$, then for a periodic $V$, since $\sigma(A)$ is pure absolutely continuous, $a_{0} \notin \sigma_{p}(A)$ and hence $0 \notin \sigma_{p}(\mathscr{O})$ as in $\left(g_{4}\right)$. Model nonlinearities which appear in Physics of propagations of laser beams in nonlinear medium with saturations are for instance

$$
g(s)=\frac{s^{3}}{1+a_{0}^{-1} s^{2}} \quad \text { and } \quad g(s)=\left(a_{0}-\frac{1}{\exp s^{2}}\right) s .
$$

Remark 3.3. Due to $\left(g_{1}\right)-\left(g_{2}\right)$, given $\varepsilon>0$ and $2 \leq p \leq 2^{*}$ there exists a constant $C_{\varepsilon}>0$ such that

$$
|g(x, s)| \leq \varepsilon|s|+C_{\varepsilon}|s|^{p-1},
$$

and hence

$$
|G(x, s)| \leq \frac{\varepsilon}{2}|s|^{2}+\frac{C_{\varepsilon}}{p}|s|^{p},
$$

for all $s \in \mathbb{R}$, and for all $x \in \mathbb{R}^{N}$.

\subsection{Variational Setting}

Let $I: H^{1}\left(\mathbb{R}^{N}\right) \rightarrow \mathbb{R}$ be the functional associated to problem $(3.1)$, then $I$ is given by

$$
I(u)=\frac{1}{2}(A u, u)_{L^{2}\left(\mathbb{R}^{N}\right)}-\int_{\mathbb{R}^{N}} G(x, u) d x .
$$

Note that in view of $\left(V_{1}\right)_{r}$ and $\left(g_{1}\right)-\left(g_{2}\right), I: H^{1}\left(\mathbb{R}^{N}\right) \rightarrow \mathbb{R}$ is well defined and $I \in C^{1}\left(H^{1}\left(\mathbb{R}^{N}\right), \mathbb{R}\right)$. Thus, as usual, a weak solution to $\left(P_{r}\right)$ is a critical point of $I: H^{1}\left(\mathbb{R}^{N}\right) \rightarrow \mathbb{R}$, a function $u \in H^{1}\left(\mathbb{R}^{N}\right)$ such that for all $v \in H^{1}\left(\mathbb{R}^{N}\right)$

$$
I^{\prime}(u) v=(A u, v)_{L^{2}\left(\mathbb{R}^{N}\right)}-\int_{\mathbb{R}^{N}} g(x, u(x)) v(x) d x=0 .
$$

Nevertheless, since $V$ and $G$ are radial functions, in order to apply Theorem 1.4 , it is convenient to define $E:=H_{\text {rad }}^{1}\left(\mathbb{R}^{N}\right)$, which is the Hilbert subspace of all radial symmetric functions in $H^{1}\left(\mathbb{R}^{N}\right)$ and consider $I: E \rightarrow \mathbb{R}$. In fact, functions in $E$ satisfy special properties that make true all necessary hypotheses on $I: E \rightarrow \mathbb{R}$, for example, recall that by Strauss [51] (cf. also [8] Theorem A.I'.) $E$ is compactly embedded in $L^{\beta}\left(\mathbb{R}^{N}\right)$, for any $\beta \in\left(2,2^{*}\right)$.

Defining $Q_{A}: E \rightarrow \mathbb{R}$ by $Q_{A}(u):=\int_{\mathbb{R}^{N}}|\nabla u(x)|^{2} d x+\int_{\mathbb{R}^{N}} V(x) u^{2}(x) d x=\frac{1}{2}(A u, u)_{L^{2}\left(\mathbb{R}^{N}\right)}$, it is a continuous quadratic form on $E$. Since $E^{0}, E^{-}, E^{+}$are the closed subspaces of $E$ on which 
$Q_{A}$ is null, negative and positive definite, then as noticed before $E=E^{0} \oplus E^{-} \oplus E^{+}$. Moreover, if $B_{Q_{A}}[u, v]=(A u, v)_{L^{2}\left(\mathbb{R}^{N}\right)}$ for all $u, v \in E$, is the bilinear form associated to $Q_{A}$ and $u, v$ belong to distinct such subspaces, then $B_{Q_{A}}[u, v]=0$ and $Q_{A}(u+v)=Q_{A}(u)+Q_{A}(v)$. In addition $E^{0}, E^{-}, E^{+}$are mutually orthogonal in the $L^{2}\left(\mathbb{R}^{N}\right)$-inner product. Hence, for $u=u^{0}+u^{+}+u^{-} \in E$, it is suitable to take as a norm in $E$ the expression

$$
\|u\|^{2}=\|u\|_{E}^{2}:=\left\|u^{0}\right\|_{L^{2}\left(\mathbb{R}^{N}\right)}^{2}+Q_{A}\left(u^{+}\right)-Q_{A}\left(u^{-}\right)
$$

which is equivalent to the standard norm of $H^{1}\left(\mathbb{R}^{N}\right)$. With the associated inner product, obtained by means of $B_{Q_{A}}[u, v]$, this norm makes $E$ a Hilbert space with orthogonal subspaces $E^{0}, E^{+}, E^{-}$. In fact, by $\left(V_{2}\right)_{r}$ and Remark 3.1 for all $u^{+} \in E^{+}$and for all $u^{-} \in E^{-}$it yields

$$
\sigma^{+}\left\|u^{+}\right\|_{L^{2}\left(\mathbb{R}^{N}\right)}^{2} \leq \int_{\mathbb{R}^{N}}\left(\left|\nabla u^{+}(x)\right|^{2}+V(x)\left(u^{+}(x)\right)^{2}\right) d x=\left\|u^{+}\right\|^{2},
$$

and

$$
-\sigma^{-}|| u^{-}\left\|_{L^{2}\left(\mathbb{R}^{N}\right)}^{2} \leq-\int_{\mathbb{R}^{N}}\left(\left|\nabla u^{-}(x)\right|^{2}+V(x)\left(u^{-}(x)\right)^{2}\right) d x=\right\| u^{-} \|^{2},
$$

which ensures that the norm chosen above is equivalent to the standard norm in $H_{r a d}^{1}\left(\mathbb{R}^{N}\right)$, once $E^{0}=\operatorname{ker}(A)$ is finite dimensional.

Observe that, $I(u)=Q_{A}(u)-\int_{\mathbb{R}^{N}} G(x, u(x)) d x$, for all $u \in E$ and since $E$ is a subspace of $H^{1}\left(\mathbb{R}^{N}\right), I \in C^{1}(E, \mathbb{R})$. Moreover, $I$ is indefinite on $E$, henceforth the goal is to apply Theorem 1.4 so as to get a critical point to $I$ restricted to $E$, and by applying the Principle of Symmetric Criticality (cf. [38]) conclude the critical point is actually a critical point to $I: H^{1}\left(\mathbb{R}^{N}\right) \rightarrow \mathbb{R}$, namely a weak solution to $\left(P_{r}\right)$. The main result of this chapter is stated below.

Theorem 3.1. Suppose $\left(V_{1}\right)_{r}-\left(V_{2}\right)_{r}$ and $\left(g_{1}\right)-\left(g_{5}\right)$ hold true. Then problem $\left(P_{r}\right)$ in 3.1 possess a radial, nontrivial, weak solution in $H^{1}\left(\mathbb{R}^{N}\right)$.

In order to show that $I$ satisfies $\left(I_{1}\right)$ of Theorem 1.4 , set $E_{1}:=E^{+}$and $E_{2}:=E^{-} \oplus E^{0}$, then it yields $E_{2}^{\perp}=E_{1}$. Now, define $L_{i}: E_{i} \rightarrow E_{i}$, for all $u \in E_{i}$, as given by

$$
\left(L_{i} u, v\right)_{E}=Q_{A}^{\prime}(u) v=B_{Q_{A}}[u, v]=(A u, v)_{L^{2}\left(\mathbb{R}^{N}\right)},
$$

for all $v \in E_{i}, i=1,2$, where $Q_{A}^{\prime}(u) v$ denotes Fréchet derivative of $Q_{A}$ at $u$ acting on $v$. Hence, $L=L_{1}+L_{2}: E_{1} \oplus E_{2} \rightarrow E_{1} \oplus E_{2}$ is a well defined, linear, bounded operator and satisfies

$$
Q_{A}(u)=\frac{1}{2}(A u, u)_{L^{2}\left(\mathbb{R}^{N}\right)}=\frac{1}{2} Q_{A}^{\prime}(u) u=\frac{1}{2} B_{Q_{A}}[u, u]=\frac{1}{2}(L u, u)_{E} .
$$

Thus, setting $B(u):=-\int_{\mathbb{R}^{N}} G(x, u(x)) d x$, for all $u \in E$, it is possible to write

$$
I(u)=\frac{1}{2}(L u, u)+B(u)
$$


satisfying $\left(I_{1}\right)$.

\subsection{Weak Continuity and Uniform Differentiation of I}

In this section, assumption $\left(I_{2}\right)$ is proved, which ensures the necessary compactness for the problem. Next lemma is a variant of Theorem A.I. in [8] which is essential for proving that $I$ satisfies $\left(I_{2}\right)$. The original version of this result can be found in [51.

Lemma 3.1. Let $P: \mathbb{R}^{N} \times \mathbb{R} \rightarrow \mathbb{R}$ and $Q: \mathbb{R} \rightarrow \mathbb{R}$ be two continuous functions satisfying

$$
\frac{P(x, s)}{Q(s)} \rightarrow 0, \quad \text { uniformly in } x \text { as }|s| \rightarrow+\infty
$$

Let $\left(u_{n}\right)$ be a sequence of measurable functions from $\mathbb{R}^{N}$ to $\mathbb{R}$ such that

$$
\sup _{n} \int_{\mathbb{R}^{N}}\left|Q\left(u_{n}(x)\right)\right| d x<+\infty
$$

and

$$
P\left(x, u_{n}(x)\right) \rightarrow v(x) \quad \text { a. } \quad \text { e. in } \quad \mathbb{R}^{N}
$$

as $n \rightarrow+\infty$. Then for any bounded Borel set $\mathscr{B}$ one has

$$
\int_{\mathscr{B}}\left|P\left(x, u_{n}(x)\right)-v(x)\right| d x \rightarrow 0
$$

as $n \rightarrow+\infty$. If one further assumes that

$$
\frac{P(x, s)}{Q(s)} \rightarrow 0, \quad \text { uniformly in } x \text { as } s \rightarrow 0,
$$

and

$$
u_{n}(x) \rightarrow 0 \text { as }|x| \rightarrow+\infty \text {, uniformly with respect to } n \text {, }
$$

then $P\left(\cdot, u_{n}(\cdot)\right)$ converges to $v$ in $L^{1}\left(\mathbb{R}^{N}\right)$ as $n \rightarrow+\infty$.

Proof. In order to prove the first part of the proposition, it is sufficient to show that $P\left(x, u_{n}(x)\right)$ is uniformly integrable on $\mathscr{B}$. In fact, if this is case, due to 3.11

$$
\int_{\mathscr{B} \cap\left\{\left|P\left(x, u_{n}(x)\right)\right| \leq R\right\}}\left|P\left(x, u_{n}(x)\right)-v(x)\right| d x \rightarrow 0,
$$

as $n \rightarrow+\infty$, by applying Lebesgue Dominated Convergence Theorem, and the integral

$$
\int_{\mathscr{B} \cap\left\{\left|P\left(x, u_{n}(x)\right)\right|>R\right\}}\left|P\left(x, u_{n}(x)\right)\right| d x
$$


is controlled by uniform integration. By condition $(3.9)$ there exists $C>0$ such that

$$
\left|P\left(x, u_{n}(x)\right)\right| \leq C\left(1+\left|Q\left(u_{n}(x)\right)\right|\right), x \in \mathbb{R}^{N}
$$

Thus, in view of 3.10 and Fatou's Lemma, it follows that $P\left(\cdot, u_{n}(\cdot)\right)$ and $v$ are in $L^{1}(\mathscr{B})$. Moreover, since $P$ is continuous, it maps compacts sets on compact sets, hence fixed $R>0$, if for some $x \in \mathbb{R},\left|P\left(x, u_{n}(x)\right)\right|>R$, there exists $M=M(R)>0$, such that $\left|u_{n}(x)\right|>M(R)$ and $M(R) \rightarrow+\infty$ as $R \rightarrow+\infty$. Then

$$
\int_{\mathscr{B} \cap\left\{\left|P\left(x, u_{n}(x)\right)\right|>R\right\}}\left|P\left(x, u_{n}(x)\right)\right| d x \leq \int_{\mathscr{B} \cap\left\{\left|u_{n}(x)\right|>M(R)\right\}}\left|P\left(x, u_{n}(x)\right)\right| d x .
$$

Applying condition (3.9), given $\varepsilon>0$ there exist $M(R)>0$, such that $\left|u_{n}(x)\right| \geq M(R)$ implies $\left|P\left(x, u_{n}(x)\right)\right| \leq \varepsilon\left|Q\left(u_{n}(x)\right)\right|$ and $\varepsilon=\varepsilon(R) \rightarrow 0$ as $M(R) \rightarrow+\infty$. Then, there exist $\tilde{C}>0$ such that

$$
\begin{aligned}
\int_{\mathscr{B} \cap\left\{\left|P\left(x, u_{n}(x)\right)\right|>R\right\}}\left|P\left(x, u_{n}(x)\right)\right| d x & \leq \int_{\mathscr{B} \cap\left\{\left|u_{n}(x)\right|>M(R)\right\}}\left|P\left(x, u_{n}(x)\right)\right| d x \\
& \leq \varepsilon(R) \int_{\mathscr{B}}\left|Q\left(u_{n}(x)\right)\right| d x \\
& \leq \tilde{C} \varepsilon(R),
\end{aligned}
$$

which shows the uniform integrability and ensures the result.

For the second part that $P\left(\cdot, u_{n}(\cdot)\right)$ converges to $v$ in $L^{1}\left(\mathbb{R}^{N}\right)$ as $n \rightarrow+\infty$, note that in virtue of (3.13) given $\varepsilon>0$ there exists $\delta>0$ such that $|s| \leq \delta$ implies $|P(x, s)| \leq \varepsilon|Q(s)|$, uniformly in $x$. Moreover, by (3.14) given $\delta>0$ there exists $R_{0}>0$ such that $\left|u_{n}(x)\right| \leq \delta$ for all $|x| \geq R_{0}$, uniformly in $n$. Thus, $|x| \geq R_{0}$ implies $\left|P\left(x, u_{n}(x)\right)\right| \leq \varepsilon\left|Q\left(u_{n}(x)\right)\right|$, uniformly in $n$. Therefore, by Fatou's Lemma $v \in L^{1}\left(\mathbb{R}^{N}\right)$ and

$$
\int_{\left\{|x| \geq R_{0}\right\}}|v(x)| d x \leq \liminf _{n \rightarrow+\infty} \int_{\left\{|x| \geq R_{0}\right\}}\left|P\left(x, u_{n}(x)\right)\right| d x \leq \tilde{C} \varepsilon .
$$

In addition, from the first part, there exists $n_{0} \in \mathbb{N}$ such that for $n \geq n_{0}$

$$
\int_{\left\{|x|<R_{0}\right\}}\left|P\left(x, u_{n}(x)\right)-v(x)\right| d x \leq \varepsilon .
$$

Hence, for $n \geq n_{0}$ it yields

$$
\int_{\mathbb{R}^{N}}\left|P\left(x, u_{n}(x)\right)-v(x)\right| d x \leq(2 \tilde{C}+1) \varepsilon
$$

which gives the result.

By means of the previous lemma, next result holds. 
Lemma 3.2. If $g$ satisfies $\left(g_{1}\right)-\left(g_{2}\right)$, then $B$ is weakly continuous.

Proof. Let $\left(u_{n}\right) \in E$ and suppose $u_{n} \rightarrow u$ in $E$, then $\left(u_{n}\right)$ is bounded in $E$. Due to $\left(g_{1}\right)-\left(g_{2}\right)$, for $2<p<2^{*}$ one has

$$
\lim _{s \rightarrow 0} \frac{G(x, s)}{|s|^{2}}=0 \text { and } \lim _{|s| \rightarrow+\infty} \frac{G(x, s)}{|s|^{p}}=0
$$

uniformly in $x$. Hence, choosing $Q(s)=|s|^{2}+|s|^{p}$, and $P(\cdot, s)=G(\cdot, s)$, it is possible to apply Lemma 3.1. Indeed, in view of (3.15) it follows that

$$
\lim _{s \rightarrow 0} \frac{G(x, s)}{|s|^{2}+|s|^{p}}=0 \text { and } \lim _{|s| \rightarrow+\infty} \frac{G(x, s)}{|s|^{2}+|s|^{p}}=0
$$

uniformly in $x$. Then $P$ and $Q$ satisfy $(3.9)$ and (3.13). Moreover,

$$
\sup _{n} \int_{\mathbb{R}^{N}}\left(\left|u_{n}(x)\right|^{2}+\left|u_{n}(x)\right|^{p}\right) d x=\sup _{n}\left(\left\|u_{n}\right\|_{L^{2}\left(\mathbb{R}^{N}\right)}^{2}+\left\|u_{n}\right\|_{L^{p}\left(\mathbb{R}^{N}\right)}^{p}\right) \leq C<+\infty
$$

since $\left(u_{n}\right)$ is bounded in $E$ and $E$ is continuously embedded in $L^{2}\left(\mathbb{R}^{N}\right)$ and $L^{p}\left(\mathbb{R}^{N}\right)$. Hence 3.10 is satisfied. Provided that $u_{n} \rightarrow u$ in $E$ and $E$ is compactly embedded in $L^{p}\left(\mathbb{R}^{N}\right), u_{n} \rightarrow u$ in $L^{p}\left(\mathbb{R}^{N}\right)$ and $u_{n}(x) \rightarrow u(x)$ almost everywhere in $\mathbb{R}^{N}$. Thus, choosing $v(x)=G(x, u(x))$ it follows that 3.11 is satisfied. Finally, since $\left(u_{n}\right) \subset H_{\text {rad }}^{1}\left(\mathbb{R}^{N}\right)$ and $u_{n}(x) \rightarrow u(x)$ almost everywhere in $\mathbb{R}^{N}$, it yields $\lim _{|x| \rightarrow+\infty} u_{n}(x)=0$, uniformly with respect to $n$ (cf. [8] Lemma A.II.). Therefore, applying Lemma 3.1 it yields $G\left(\cdot, u_{n}(\cdot)\right)=P\left(\cdot, u_{n}(\cdot)\right) \rightarrow v=G(\cdot, u(\cdot))$ in $L^{1}\left(\mathbb{R}^{N}\right)$ as $n \rightarrow+\infty$, namely,

$$
B\left(u_{n}\right)=-\int_{\mathbb{R}^{N}} G\left(x, u_{n}(x)\right) d x \rightarrow-\int_{\mathbb{R}^{N}} G\left(x, u_{n}(x)\right)=B(u),
$$

as $n \rightarrow+\infty$ and then $B$ is weakly continuous.

Next Lemma is proved indirectly by means of the compact embeddings of $E$ on Lebesgue's spaces, as mentioned before.

Lemma 3.3. Assume that $g$ satisfies $\left(g_{1}\right)-\left(g_{2}\right)$, then $B$ is uniformly differentiable on bounded sets of $E$.

Proof. First, note that fixed $R>0$ and given $u+v, v \in B_{R} \subset E$, the closed ball centered on the origin, one has

$$
\begin{aligned}
\left|B(u+v)-B(u)-B^{\prime}(u) v\right| & =\int_{\mathbb{R}^{N}}|G(x, u(x)+v(x))-G(x, u(x))-g(x, u(x)) v(x)| d x \\
& \leq \int_{\mathbb{R}^{N}}|g(x, z(x))-g(x, u(x))||v(x)| d x \\
& \leq C_{2}|| \xi\left\|_{L^{2}\left(\mathbb{R}^{N}\right)}\right\| v \|,
\end{aligned}
$$


where $\xi(x):=|g(x, z(x))-g(x, u(x))|$ and $z(x)=u(x)+\theta(x) v(x)$, with $0 \leq \theta(x) \leq 1$ given by Mean Value Theorem and $C_{2}>0$ is the constant given by the continuous embedding $E \hookrightarrow L^{2}\left(\mathbb{R}^{N}\right)$.

With the purpose of proving that $B$ is uniformly differentiable on bounded sets of $E$, given $\varepsilon>0$ it is sufficient to show there exist $\delta>0$ such that $C_{2}\|\xi\|_{L^{2}\left(\mathbb{R}^{N}\right)} \leq \varepsilon$ for all $u+v, v \in B_{R}$ with $\|v\| \leq \delta$. Seeking a contradiction, suppose that it is not the case, then there exist $R_{0}, \varepsilon_{0}>0$ such that for all $\delta>0$ there are $u_{\delta}+v_{\delta}, v_{\delta} \in B_{R_{0}}$ with $\left\|v_{\delta}\right\| \leq \delta$ and $C_{2}\|\xi\|_{L^{2}\left(\mathbb{R}^{N}\right)}>\varepsilon_{0}$. Thus, it is possible to obtain for all $n \in \mathbb{N}$ and $\delta=\frac{1}{n}$ functions $u_{n}+v_{n}, v_{n} \in B_{R_{0}}$ such that $\left\|v_{n}\right\| \leq \frac{1}{n}$ and $C_{2}\left\|\xi_{n}\right\|_{L^{2}\left(\mathbb{R}^{N}\right)}>\varepsilon_{0}$, for $\xi_{n}(x)=\left|g\left(x, z_{n}(x)\right)-g\left(x, u_{n}(x)\right)\right|$, with $z_{n}=u_{n}+\theta_{n} v_{n}$, and $0 \leq \theta_{n} \leq 1$ depending on $u_{n}$ and $v_{n}$ as before. Since $v_{n} \rightarrow 0$ in $E$, then $v_{n} \rightarrow 0$ in $L^{2}\left(\mathbb{R}^{N}\right), v_{n}(x) \rightarrow 0$ almost everywhere in $\mathbb{R}^{N}$ and there exists $\psi \in L^{2}\left(\mathbb{R}^{N}\right)$ such that $\left|v_{n}(x)\right| \leq \psi(x)$ almost everywhere in $\mathbb{R}^{N}$. Furthermore, since $\left(u_{n}\right) \subset B_{R_{0}}$, it is bounded in $E$, then $u_{n} \rightarrow u$ in $E$ up to subsequences, then $u_{n} \rightarrow u$ in $L_{l o c}^{2}\left(\mathbb{R}^{N}\right)$ up to subsequences, hence $u_{n}(x) \rightarrow u(x)$ almost everywhere in $\mathbb{R}^{N}$ and fixed $B_{r}(0) \subset \mathbb{R}^{N}$ there exists $\varphi_{r} \in L^{2}\left(B_{r}(0)\right)$ such that $\left|u_{n}(x)\right| \leq \varphi_{r}(x)$ almost everywhere in $B_{r}(0)$ up to subsequences. In addition, $z_{n}(x) \rightarrow u$ in $E$ up to subsequences, then $z_{n} \rightarrow u$ in $L_{l o c}^{2}\left(\mathbb{R}^{N}\right)$ up to subsequences, hence $z_{n}(x) \rightarrow u(x)$ almost everywhere in $\mathbb{R}^{N}$, which implies that $\xi_{n}(x) \rightarrow 0$, almost everywhere in $\mathbb{R}^{N}$, provided that $g$ is continuous. Moreover, in view of Remark 3.3 with $p=2$, it yields

$$
\begin{aligned}
\left|\xi_{n}(x)\right|^{2} & \leq 2\left[\left|g\left(x, z_{n}(x)\right)\right|^{2}+\left|g\left(x, u_{n}(x)\right)\right|^{2}\right] \\
& \leq 2\left[C^{2}\left|z_{n}(x)\right|^{2}+C^{2}\left|u_{n}(x)\right|^{2}\right] \\
& \leq 2 C^{2}\left[2\left(\left|u_{n}(x)\right|^{2}+\left|v_{n}(x)\right|^{2}\right)+\left|u_{n}(x)\right|^{2}\right] \\
& \leq 2 C^{2}\left[3\left|u_{n}(x)\right|^{2}+2\left|v_{n}(x)\right|^{2}\right] \\
& \leq 6 C^{2}\left[\varphi_{r}^{2}(x)+\psi^{2}(x)\right]
\end{aligned}
$$

almost everywhere in $B_{r}(0)$. Since $\varphi_{r}^{2}+\psi^{2} \in L^{1}\left(B_{r}(0)\right)$, applying Lebesgue Dominated Convergence Theorem, it yields

$$
\int_{B_{r}(0)}\left|\xi_{n}(x)\right|^{2} d x \rightarrow 0
$$

as $n \rightarrow+\infty$. On the other hand, since $\left(z_{n}\right) \subset H_{\text {rad }}^{1}\left(\mathbb{R}^{N}\right)$ and $\left(u_{n}\right) \subset H_{\text {rad }}^{1}\left(\mathbb{R}^{N}\right)$ are bounded sequences, it follows that

$$
\lim _{|x| \rightarrow+\infty} z_{n}(x)=\lim _{|x| \rightarrow+\infty} u_{n}(x)=0,
$$

uniformly with respect to $n$, by the characterization of decay of radial functions (cf. for instance [8] Radial Lemma A.II). Hence, given $\varsigma>0$, there exists $r>0$ such that $|x| \geq r$ implies $\left|z_{n}(x)\right|,\left|u_{n}(x)\right| \leq \varsigma$ for all $n \in \mathbb{N}$. Moreover, given $\vartheta>0$ by $\left(g_{1}\right)$ there exists $\varsigma>0$ sufficiently 
small such that $|g(x, s)| \leq \vartheta|s|$ for all $|s| \leq \varsigma$. Hence, for $r>0$ sufficiently large, it yields

$$
\left|g\left(x, z_{n}(x)\right)\right| \leq \vartheta\left|z_{n}(x)\right| \text { and }\left|g\left(x, u_{n}(x)\right)\right| \leq \vartheta\left|u_{n}(x)\right|
$$

for all $|x| \geq r$ and since $\left(z_{n}\right)$ and $\left(u_{n}\right)$ are bounded in $L^{2}\left(\mathbb{R}^{N}\right)$, it yields

$$
\begin{aligned}
\int_{\mathbb{R}^{N} \backslash B_{r}(0)}\left|\xi_{n}(x)\right|^{2} d x & \leq 2 \int_{\mathbb{R}^{N} \backslash B_{r}(0)}\left[\left|g\left(x, z_{n}(x)\right)\right|^{2}+\left|g\left(x, u_{n}(x)\right)\right|^{2}\right] d x \\
& \leq 2 \vartheta \int_{\mathbb{R}^{N} \backslash B_{r}(0)}\left(\left|z_{n}(x)\right|^{2}+\left|u_{n}(x)\right|^{2}\right) d x \\
& \leq 2 \vartheta \sup _{n}\left(\left\|z_{n}\right\|_{L^{2}\left(\mathbb{R}^{N}\right)}^{2}+\left\|u_{n}\right\|_{L^{2}\left(\mathbb{R}^{N}\right)}^{2}\right) \\
& \leq C \vartheta \\
& <\frac{1}{2}\left(\frac{\varepsilon_{0}}{C_{2}}\right)^{2}
\end{aligned}
$$

for $\vartheta$ sufficiently small. Therefore, combining (3.20) and (3.21) it follows that as $n \rightarrow+\infty$

$$
\left(\frac{\varepsilon_{0}}{C_{2}}\right)^{2}<\left\|\xi_{n}\right\|_{L^{2}\left(\mathbb{R}^{N}\right)}^{2}=\int_{\mathbb{R}^{N}}\left|\xi_{n}(x)\right|^{2} d x \leq o_{n}(1)+\frac{1}{2}\left(\frac{\varepsilon_{0}}{C_{2}}\right)^{2} .
$$

Thus, passing to the limit as $n \rightarrow+\infty$ it yields a contradiction. Therefore, the result holds.

\subsection{Linking Geometry}

Now, the linking structure in $\left(I_{3}\right)$ is proved. To do so, set $S:=\left(\partial B_{\rho} \cap E_{1}\right)$ and

$$
Q:=\left\{r e+u_{2}: r \geq 0, u_{2} \in E_{2},\left\|r e+u_{2}\right\| \leq r_{1}\right\}
$$

where $0<\rho<r_{1}$ are constants and $e \in E_{1},\|e\|=1$, is chosen suitably. Indeed, due to the strict inequality in $\left(g_{3}\right)$ and from Remark 3.1 it is possible to choose $e \in E_{1}$ a unitary vector given by the spectral family of operator $A$ and $\varepsilon>0$ small enough satisfying

$$
\begin{aligned}
1 & =\|e\|^{2}=Q_{A}(e)=\frac{1}{2}(A e, e) L_{L^{2}\left(\mathbb{R}^{N}\right)} \\
& \leq \frac{1}{2}\left(\sigma^{+}+\varepsilon\right)\|e\|_{L^{2}\left(\mathbb{R}^{N}\right)}^{2} \\
& <\frac{1}{2} a_{0}\|e\|_{L^{2}\left(\mathbb{R}^{N}\right)}^{2} \\
& \leq \frac{1}{2} \int_{\mathbb{R}^{N}} h(x) e^{2}(x) d x
\end{aligned}
$$

Choosing such an $e$, by means of 3.22 it is possible to show that for sufficiently large $r_{1}>0$ one has $\left.I\right|_{S} \geq \alpha>0$ for some $\alpha>0$, and $\left.I\right|_{\partial Q} \leq 0$ holds true. Moreover, as noticed before, $S$ and $Q$ "link". Hence, $I$ satisfies $\left(I_{3}\right)$ for some $\alpha>0, \omega=0$ and arbitrary $v \in E_{2}$. 
Lemma 3.4. Under the hypotheses $\left(V_{1}\right)_{r}-\left(V_{2}\right)_{r}$ on $V$ and $\left(g_{1}\right)-\left(g_{3}\right)$ on $g$, I satisfies $\left(I_{3}\right)$.

Proof. Since $S \subset E_{1}$, by Remark 3.3 , for $2<p<2^{*}$ and for all $u_{1} \in S$, it yields

$$
\begin{aligned}
I\left(u_{1}\right) & =\frac{1}{2}\left\|u_{1}\right\|^{2}-\int_{\mathbb{R}^{N}} G\left(x, u_{1}(x)\right) d x \\
& \geq \frac{1}{2} \rho^{2}-\int_{\mathbb{R}^{N}}\left(\frac{\varepsilon}{2}\left|u_{1}(x)\right|^{2}+\frac{C_{\varepsilon}}{p}\left|u_{1}(x)\right|^{p}\right) d x \\
& \geq \frac{1}{2} \rho^{2}-\left(\frac{\varepsilon}{2} C_{2}^{2}|| u_{1}\left\|^{2}+\frac{C_{\varepsilon}}{p} C_{p}^{p}\right\| u_{1} \|^{p}\right) \\
& =\rho^{2}\left[\frac{1}{2}\left(1-\varepsilon C_{2}^{2}\right)-\frac{C_{\varepsilon}}{p} C_{p}^{p} \rho^{p-2}\right] \\
& \geq \rho^{2}\left(d_{1}-d_{2}\right)=\alpha>0,
\end{aligned}
$$

where $\varepsilon, \rho$ are sufficiently small, such that $1>\varepsilon C_{2}^{2}$ and also

$$
d_{1}:=\frac{1}{2}\left(1-\varepsilon C_{2}^{2}\right)>\frac{C_{\varepsilon}}{p} C_{p}^{p} \rho^{p-2}=: d_{2} .
$$

Therefore, from (3.23), $\left(I_{3}\right)(i)$ holds for $I$.

In order to prove that $I$ satisfies $\left(I_{3}\right)(i i)$ in Theorem 1.4, with $\omega=0$, observe that $I(u) \leq 0$, for all $u \in E_{2}=E^{-} \oplus E^{0}$, then it suffices to show that $I(r e+u) \leq 0$ for $r>0, u \in E_{2}$ and $\|r e+u\| \geq r_{1}$, for some $r_{1}>0$ large enough. Arguing indirectly assume that for some sequence $\left(r_{n} e+u_{n}\right) \subset \mathbb{R}^{+} e \oplus E_{2}$ with $\left\|r_{n} e+u_{n}\right\| \rightarrow+\infty, I\left(r_{n} e+u_{n}\right)>0$ holds, for all $n \in \mathbb{N}$. Seeking a contradiction, set

$$
\tilde{u}_{n}:=\frac{r_{n} e+u_{n}}{\left\|r_{n} e+u_{n}\right\|}=s_{n} e+w_{n}
$$

where $s_{n} \in \mathbb{R}^{+}, w_{n}=w_{n}^{-}+w_{n}^{0} \in E_{2}=E^{-} \oplus E^{0}$ and $\left\|\tilde{u}_{n}\right\|=1$. Provided that $\left(\tilde{u}_{n}\right)$ is bounded, up to subsequences it follows that $\tilde{u}_{n} \rightarrow \tilde{u}=s e+w$ in $E$, hence $\tilde{u}_{n} \rightarrow u$ in $L_{l o c}^{2}\left(\mathbb{R}^{N}\right)$. Then, up to subsequences, $\tilde{u}_{n}(x) \rightarrow \tilde{u}(x)$ almost everywhere in $\mathbb{R}^{N}, s_{n} \rightarrow s$ in $\mathbb{R}^{+}, w_{n}^{-} \rightarrow w$ in $E$, and $w_{n}^{0} \rightarrow w^{0}$ in $E$, since $s_{n}, w_{n}^{-}$and $w_{n}^{0}$ are also bounded, $\left(w_{n}^{0}\right) \subset E^{0}$ and $E^{0}$ is finite dimensional. Noting that $1=\left\|s_{n} e+w_{n}\right\|^{2}=s_{n}^{2}+\left\|w_{n}^{-}\right\|^{2}+\left\|w_{n}^{0}\right\|^{2}$, it follows that $0 \leq s_{n}^{2} \leq 1$, and it yields

$$
\begin{aligned}
\frac{I\left(r_{n} e+u_{n}\right)}{\left\|r_{n} e+u_{n}\right\|^{2}} & =s_{n}^{2}\|e\|^{2}-\left\|w_{n}^{-}\right\|^{2}-\int_{\mathbb{R}^{N}} \frac{G\left(x, r_{n} e(x)+u_{n}(x)\right)}{\left\|r_{n} e+u_{n}\right\|^{2}} d x \\
& =2 s_{n}^{2}-1-\left\|w_{n}^{0}\right\|^{2}-\int_{\mathbb{R}^{N}} \frac{G\left(x, r_{n} e(x)+u_{n}(x)\right)}{\left\|r_{n} e+u_{n}\right\|^{2}} d x>0,
\end{aligned}
$$

hence $\frac{1}{2} \leq s^{2} \leq 1$. Moreover, from 3.22 it is possible to choose a bounded domain $\Omega \subset \mathbb{R}^{N}$, such that

$$
1<\frac{1}{2} \int_{\Omega} h(x) e^{2}(x) d x .
$$


Hence,

$$
\begin{aligned}
0 & >s^{2}-s^{2} \frac{1}{2} \int_{\Omega} h(x) e^{2}(x) d x \\
& \geq s^{2}\left(1-\frac{1}{2} \int_{\Omega} h(x) e^{2}(x) d x\right)-\left(1+\left\|w^{0}\right\|^{2}-s^{2}\right)-\frac{1}{2} \int_{\Omega} h(x) w^{2}(x) d x \\
& =s^{2}\left(2-\frac{1}{2} \int_{\Omega} h(x) e^{2}(x) d x\right)-1-\left\|w^{0}\right\|^{2}-\frac{1}{2} \int_{\Omega} h(x) w^{2}(x) d x .
\end{aligned}
$$

On the other hand, from assumptions $\left(g_{1}\right)-\left(g_{2}\right)$ and since $\tilde{u}_{n}$ is convergent in $L^{2}(\Omega)$, there exists some $\psi \in L^{1}(\Omega)$ such that

$$
\left|\frac{G\left(\cdot, r_{n} e(\cdot)+u_{n}(\cdot)\right)}{|| r_{n} e+u_{n}||^{2}}\right| \leq r_{\infty}\left|\tilde{u}_{n}(\cdot)\right|^{2} \leq \psi(\cdot) \in L^{1}(\Omega) .
$$

Moreover, provided that $\left\|r_{n} e+u_{n}\right\| \rightarrow+\infty$, and $\tilde{u}_{n}(x) \rightarrow \tilde{u}(x) \neq 0$, almost everywhere in $\operatorname{supp}(\tilde{u})$, it follows that $u_{n}(x)=\tilde{u}_{n}(x)\left\|r_{n} e+u_{n}(x)\right\| \rightarrow+\infty$ almost everywhere in $\operatorname{supp}(\tilde{u})$, as $n \rightarrow+\infty$, hence

$$
\frac{G\left(x, r_{n} e(x)+u_{n}(x)\right)}{\left\|r_{n} e+u_{n}\right\|^{2}}=\frac{G\left(x, \tilde{u}_{n}(x)\left\|r_{n} e+u_{n}\right\|\right) \tilde{u}_{n}^{2}(x)}{\tilde{u}_{n}^{2}(x)\left\|r_{n} e+u_{n}\right\|^{2}} \rightarrow \frac{1}{2} h(x) \tilde{u}^{2}(x)
$$

almost everywhere in $\operatorname{supp}(\tilde{z})$ as $n \rightarrow+\infty$. Note that, $\operatorname{supp}(\tilde{u}) \neq \emptyset$, because $\tilde{u}=s e+w$, with $\operatorname{supp}(e) \neq \emptyset$ and $(e, w)_{L^{2}\left(\mathbb{R}^{N}\right)}=0$. Thus, by Lebesgue Dominated Convergence Theorem,

$$
\int_{\Omega} \frac{G\left(x, r_{n} e(x)+u_{n}(x)\right)}{\left\|r_{n} e+u_{n}\right\|^{2}} d x \rightarrow \frac{1}{2} \int_{\Omega} h(x)(s e(x)+w(x))^{2} d x
$$

as $n \rightarrow+\infty$. From $(3.24)$ one has

$$
2 s_{n}^{2}-1-\left\|w_{n}^{0}\right\|^{2}-\int_{\Omega} \frac{G\left(x, r_{n} e(x)+u_{n}(x)\right)}{\left\|r_{n} e+u_{n}\right\|^{2}} d x>0 .
$$

Passing to the limit as $n \rightarrow+\infty$, it yields

$$
\begin{aligned}
0 & \leq 2 s^{2}-1-\left\|w^{0}\right\|^{2}-\frac{1}{2} \int_{\Omega} h(x)\left(s^{2} e^{2}(x)+w^{2}(x)\right) d x \\
& =s^{2}\left(2-\frac{1}{2} \int_{\Omega} h(x) e^{2}(x) d x\right)-1-\left\|w^{0}\right\|^{2}-\frac{1}{2} \int_{\Omega} h(x) w^{2}(x) d x
\end{aligned}
$$

which is contrary to 3.25 . Therefore the result holds. 


\subsection{Boundedness of Cerami Sequences}

Finally, in this section the boundedness of Cerami sequences is verified, which shows that hypothesis $\left(I_{4}\right)$ holds true, and then the existence result for problem 3.1$)$ is proved.

For the boundedness of Cerami sequences, standard arguments are applied and hypotheses $\left(g_{4}\right)$ and $\left(g_{5}\right)$ are finally exploited. It is important to point out that these assumptions are only used in order to prove next lemma, since the special properties of radial functions are not sufficient when problem $\left(P_{r}\right)$ is treated in $\mathbb{R}^{N}$.

Lemma 3.5. Suppose that $V$ satisfies $\left(V_{1}\right)_{r}-\left(V_{2}\right)_{r}$ and $g$ satisfies $\left(g_{1}\right)-\left(g_{5}\right)$, then I satisfies $\left(I_{4}\right)$.

Proof. Let $b>0$ be an arbitrary constant, and take $\left(u_{n}\right) \subset I^{-1}([c-b, c+b])$ such that $\left(1+\left\|u_{n}\right\|\right)\left\|I^{\prime}\left(u_{n}\right)\right\| \rightarrow 0$, it is necessary to show that $\left(u_{n}\right)$ is bounded. Suppose by contradiction that $\left\|u_{n}\right\| \rightarrow+\infty$, up to subsequences. Setting $\tilde{u}_{n}:=\frac{u_{n}}{\left\|u_{n}\right\|}$, it is bounded, hence $\tilde{u}_{n} \rightarrow \tilde{u}$ in $E$ and $\tilde{u}_{n} \rightarrow \tilde{u}$ in $L^{\beta}\left(\mathbb{R}^{N}\right)$, for $\beta \in\left(2,2^{*}\right)$, due to the compact embeddings previously mentioned (cf. [51] and [8]). Writing $u_{n}=u_{n}^{+}+u_{n}^{-}+u_{n}^{0} \in E^{+} \oplus E^{-} \oplus E^{0}$, by the choice of $u_{n}$ it satisfies

$$
\begin{aligned}
o_{n}(1) & =I^{\prime}\left(u_{n}\right) \frac{u_{n}^{+}}{\left\|u_{n}\right\|^{2}} \\
& =\frac{1}{\left\|u_{n}\right\|} I^{\prime}\left(u_{n}\right) \tilde{u}_{n}^{+} \\
& =\left\|\tilde{u}_{n}^{+}\right\|^{2}-\int_{\mathbb{R}^{N}} \frac{g\left(x, u_{n}(x)\right)}{u_{n}(x)} \tilde{u}_{n}(x) \tilde{u}_{n}^{+}(x) d x .
\end{aligned}
$$

and

$$
\begin{aligned}
o_{n}(1) & =I^{\prime}\left(u_{n}\right) \frac{u_{n}^{-}}{\left\|u_{n}\right\|^{2}} \\
& =\frac{1}{\left\|u_{n}\right\|} I^{\prime}\left(u_{n}\right) \tilde{u}_{n}^{-} \\
& =-\left\|\tilde{u}_{n}^{-}\right\|^{2}-\int_{\mathbb{R}^{N}} \frac{g\left(x, u_{n}(x)\right)}{u_{n}(x)} \tilde{u}_{n}(x) \tilde{u}_{n}^{-}(x) d x .
\end{aligned}
$$

Subtracting (3.28) from (3.27), and using that $1=\left\|\tilde{u}_{n}^{+}\right\|^{2}+\left\|\tilde{u}_{n}^{-}\right\|^{2}+\left\|\tilde{u}_{n}^{0}\right\|^{2}$, it yields

$$
o_{n}(1)=1-\left\|\tilde{u}_{n}^{0}\right\|^{2}-\int_{\mathbb{R}^{N}} \frac{g\left(x, u_{n}(x)\right)}{u_{n}(x)}\left[\left(\tilde{u}_{n}^{+}(x)\right)^{2}-\left(\tilde{u}_{n}^{-}(x)\right)^{2}\right] d x .
$$

Provided that $\left(\tilde{u}_{n}^{0}\right) \subset E^{0}$, which is finite dimensional, then the weak convergence implies that $\tilde{u}_{n}^{0} \rightarrow \tilde{u}^{0}$ in $E$.

On the other hand, given $\varphi \in C_{0}^{\infty}\left(\mathbb{R}^{N}\right)$ and setting $\operatorname{supp}(\varphi):=K$, since $\tilde{u}_{n} \rightarrow \tilde{u}$ in 
$L^{2}(K)$, by applying Lebesgue Dominated Convergence Theorem it follows that

$$
\int_{K} \frac{g\left(x, u_{n}(x)\right)}{u_{n}(x)} \tilde{u}_{n}(x) \varphi(x) d x=\int_{K} h(x) \tilde{u}_{n}(x) \varphi(x) d x+o_{n}(1)
$$

as $n \rightarrow+\infty$. Hence, it yields

$$
\begin{aligned}
o_{n}(1) & =\frac{I^{\prime}\left(u_{n}\right) \varphi}{\left\|u_{n}\right\|} \\
& =\frac{Q_{A}^{\prime}\left(u_{n}\right) \varphi}{\left\|u_{n}\right\|}-\int_{K} \frac{g\left(x, u_{n}(x)\right)}{u_{n}(x)} \tilde{u}_{n}(x) \varphi(x) d x \\
& =\left(A \tilde{u}_{n}, \varphi\right)_{L^{2}\left(\mathbb{R}^{N}\right)}-\int_{K} h(x) \tilde{u}_{n}(x) \varphi(x) d x+o_{n}(1) \\
& =\left(\mathscr{O} \tilde{u}_{n}, \varphi\right)_{L^{2}\left(\mathbb{R}^{N}\right)}+o_{n}(1) \\
& =(\mathscr{O} \tilde{u}, \varphi)_{L^{2}\left(\mathbb{R}^{N}\right)}+o_{n}(1) .
\end{aligned}
$$

Due to 3.30 , if $\tilde{u} \neq 0$ it is an eigenvector of $\mathscr{O}$ with eigenvalue 0 . Nevertheless, from $\left(g_{4}\right)$, $0 \notin \sigma_{p}(\mathscr{O})$ and hence $\tilde{u}=0$. It means that $\tilde{u}^{+}=\tilde{u}^{-}=\tilde{u}^{0}=0$ and thus $\tilde{u}_{n}^{ \pm} \rightarrow 0$ in $L^{\beta}\left(\mathbb{R}^{N}\right)$, for $\beta \in\left(2,2^{*}\right)$, due to the compact embeddings, and $\tilde{u}_{n}^{0} \rightarrow 0$ in $E$. Hence, from 3.29 one arrives at

$$
\int_{\mathbb{R}^{N}} \frac{g\left(x, u_{n}(x)\right)}{u_{n}(x)}\left[\left(\tilde{u}_{n}^{+}(x)\right)^{2}-\left(\tilde{u}_{n}^{-}(x)\right)^{2}\right] d x=1+o_{n}(1) .
$$

Now, defining $\Omega_{n}:=\left\{x \in \mathbb{R}^{N}: \frac{\left|g\left(x, u_{n}(x)\right)\right|}{\left|u_{n}(x)\right|} \leq \sigma_{0}-\delta_{0}\right\} \subset \mathbb{R}^{N}$ for all $n \in \mathbb{N}$ and for $\delta_{0}>0$ given by $\left(g_{5}\right)$, since $\sigma_{0} \leq \sigma^{ \pm}$then $\sigma_{0}\|w\|_{L^{2}\left(\mathbb{R}^{N}\right)}^{2} \leq\|w\|^{2}$ for all $w \in E^{+} \oplus E^{-}$, it follows that

$$
\begin{aligned}
\int_{\Omega_{n}} \frac{\left|g\left(x, u_{n}(x)\right)\right|}{\left|u_{n}(x)\right|}\left|\left(\tilde{u}_{n}^{+}(x)\right)^{2}-\left(\tilde{u}_{n}^{-}(x)\right)^{2}\right| d x & \leq\left(\sigma_{0}-\delta_{0}\right) \int_{\Omega_{n}}\left[\left(\tilde{u}_{n}^{+}(x)\right)^{2}+\left(\tilde{u}_{n}^{-}(x)\right)^{2}\right] d x \\
& \leq\left(\sigma_{0}-\delta_{0}\right)\left\|\tilde{u}_{n}^{+}+\tilde{u}_{n}^{-}\right\|_{L^{2}\left(\mathbb{R}^{N}\right)}^{2} \\
& \leq \frac{\left(\sigma_{0}-\delta_{0}\right)}{\sigma_{0}}\left\|\tilde{u}_{n}^{+}+\tilde{u}_{n}^{-}\right\|^{2} \\
& \leq 1-\frac{\delta_{0}}{\sigma_{0}}
\end{aligned}
$$

Combining 3.31 and 3.32 one has

$$
\begin{aligned}
& \int_{\mathbb{R}^{N} \backslash \Omega_{n}} \frac{\left|g\left(x, u_{n}(x)\right)\right|}{\left|u_{n}(x)\right|}\left|\left(\tilde{u}_{n}^{+}(x)\right)^{2}-\left(\tilde{u}_{n}^{-}(x)\right)^{2}\right| d x \\
\geq & 1-\int_{\Omega_{n}} \frac{\left|g\left(x, u_{n}(x)\right)\right|}{\left|u_{n}(x)\right|}\left|\left(\tilde{u}_{n}^{+}(x)\right)^{2}-\left(\tilde{u}_{n}^{-}(x)\right)^{2}\right| d x+o_{n}(1) \\
\geq & \frac{\delta_{0}}{\sigma_{0}}+o_{n}(1),
\end{aligned}
$$


and thus,

$$
\liminf _{n \rightarrow+\infty} \int_{\mathbb{R}^{N} \backslash \Omega_{n}} \frac{\left|g\left(x, u_{n}(x)\right)\right|}{\left|u_{n}(x)\right|}\left|\left(\tilde{u}_{n}^{+}(x)\right)^{2}-\left(\tilde{u}_{n}^{-}(x)\right)^{2}\right| d x \geq \frac{\delta_{0}}{\sigma_{0}}
$$

Furthermore, since $\left|\frac{g(x, s)}{s}\right|$ is bounded, applying Hölder Inequality for $\beta \in\left(2,2^{*}\right)$ one has

$$
\int_{\mathbb{R}^{N} \backslash \Omega_{n}} \frac{\left|g\left(x, u_{n}(x)\right)\right|}{\left|u_{n}(x)\right|}\left|\left(\tilde{u}_{n}^{+}(x)\right)^{2}-\left(\tilde{u}_{n}^{-}(x)\right)^{2}\right| d x \leq C\left|\mathbb{R}^{N} \backslash \Omega_{n}\right|^{\frac{\beta-2}{\beta}}\left\|\tilde{u}_{n}^{+}+\tilde{u}_{n}^{-}\right\|_{L^{\beta}\left(\mathbb{R}^{N}\right)}^{\frac{2}{\beta}},
$$

for some $C>0$. Provided that $\tilde{u}_{n}^{ \pm} \rightarrow 0$ in $L^{\beta}\left(\mathbb{R}^{N}\right)$ for $\beta \in\left(2,2^{*}\right)$, in view of 3.33 and 3.34 it yields

$$
\left|\mathbb{R}^{N} \backslash \Omega_{n}\right| \rightarrow+\infty, \quad \text { as } n \rightarrow+\infty .
$$

Nevertheless, in virtue of $\left(g_{5}\right)$ and since $\left(u_{n}\right)$ is a Cerami sequence one can find a constant $M>0$ such that

$$
M \geq I\left(u_{n}\right)-\frac{1}{2} I^{\prime}\left(u_{n}\right) u_{n}=\int_{\mathbb{R}^{N}} Q\left(x, u_{n}(x)\right) d x \geq \int_{\mathbb{R}^{N} \backslash \Omega_{n}} Q\left(x, u_{n}(x)\right) d x \geq \delta_{0}\left|\mathbb{R}^{N} \backslash \Omega_{n}\right|,
$$

which contradicts (3.35). Therefore, $\left(u_{n}\right)$ is bounded and the result holds.

Lastly, the main result of the chapter is demonstrated below.

Proof of Theorem 3.1. Provided that $I$ satisfies all assumptions $\left(I_{1}\right)-\left(I_{4}\right)$ in Theorem 1.4 , it ensures a critical point $u \in E$ of $I$, with $I(u)=c \geq \alpha>0$, hence $u$ is a non-trivial critical point to $I: E \rightarrow \mathbb{R}$. It implies that $I^{\prime}(u) v=0$, for all $v \in H_{\text {rad }}^{1}\left(\mathbb{R}^{N}\right)$. Nevertheless, the Principle of Symmetric Criticality [38] implies that $I^{\prime}(u) v=0$ for all $v \in H^{1}\left(\mathbb{R}^{N}\right)$, namely, $u$ is a critical point to $I$ as a functional defined on the whole $H^{1}\left(\mathbb{R}^{N}\right)$. Since $I \in C^{1}\left(H^{1}\left(\mathbb{R}^{N}\right), \mathbb{R}\right)$, it yields that $u$ is a nontrivial weak solution to $\left(P_{r}\right)$. In addition, since $u \in E$, it is a radial weak solution. 


\section{Chapter}

4

Non-cooperative Hamiltonian Elliptic Systems in $\mathbb{R}^{N}$

This chapter is devoted to solve non-cooperative Hamiltonian elliptic systems under hypotheses on the potentials inspired by [50].

For $N \geq 2$ consider the elliptic system $(E S)$ given by

$$
\left\{\begin{array}{l}
-\Delta u+V_{1}(x) u=F_{u}(x, u, v) \quad \text { in } \quad \mathbb{R}^{N} \\
-\Delta v+V_{2}(x) v=-F_{v}(x, u, v) \quad \text { in } \quad \mathbb{R}^{N},
\end{array}\right.
$$

where, for $i=1,2$, the potentials $V_{i} \in L_{l o c}^{\infty}\left(\mathbb{R}^{N}\right)$, satisfy

$\left(V_{1}\right)_{i}$ There exist constants $a_{i} \geq 0$ such that $V_{i}(x) \geq-a_{i}$ for all $x \in \mathbb{R}^{N}$;

$\left(V_{2}\right)_{i}$ Defining for all $\Omega \subset \mathbb{R}^{N}$ open,

$$
\nu_{i}(\Omega):=\inf \left\{\int_{\Omega}\left(|\nabla u(x)|^{2}+V_{i}(x) u^{2}(x)\right) d x: u \in H_{0}^{1}(\Omega),\|u\|_{L^{2}(\Omega)}=1\right\},
$$

assume that $\lim _{r \rightarrow+\infty} \nu_{i}\left(\mathbb{R}^{N} \backslash B_{r}\right)=+\infty$, where $B_{r}=\left\{x \in \mathbb{R}^{N}:|x| \leq r\right\}$;

$\left(V_{3}\right)_{i}$ Setting the first eigenvalue of operator $A_{i}:=-\Delta+V_{i}(x)$ in $L^{2}\left(\mathbb{R}^{N}\right)$ by

$$
\lambda_{1}^{i}:=\inf \left\{\int_{\mathbb{R}^{N}}\left(|\nabla u(x)|^{2}+V_{i}(x) u^{2}(x)\right) d x: u \in H^{1}\left(\mathbb{R}^{N}\right),\|u\|_{L^{2}\left(\mathbb{R}^{N}\right)}=1\right\},
$$

assume that $\lambda_{1}^{i}>0$; 
$(V)$ There exists $h(x) \in L_{l o c}^{\infty}\left(\mathbb{R}^{N}\right)$ and constants $\alpha_{0}>1, c_{0}>0, r_{0}>0$ such that

$$
h(x) \leq c_{0}\left[1+\left(\max _{i=1,2}\left\{0, V_{i}(x)\right\}\right)^{\frac{1}{\alpha_{0}}}\right], \quad \text { if } \quad|x| \geq r_{0} .
$$

Moreover, setting $a_{0}:=\inf _{x \in \mathbb{R}^{N}} h(x)$, assume that $a_{0}>\lambda_{1}^{1}>0$.

Remark 4.1. Note that potentials satisfying

$$
V_{i}(x) \rightarrow+\infty \quad \text { as } \quad|x| \rightarrow+\infty, \quad \text { or } \quad V_{i}(x)>V_{0}>0 \quad \text { and } \quad \frac{1}{V_{i}(x)} \in L^{1}\left(\mathbb{R}^{N}\right)
$$

or even such that

$$
\left|\Omega_{M}^{V_{i}}\right|<+\infty \text { for all } M>0,
$$

where $\Omega_{M}^{V_{i}}=\left\{x \in \mathbb{R}^{N}: V_{i}(x)<M\right\}$, also satisfy conditions $\left(V_{1}\right)_{i}-\left(V_{2}\right)_{i}$. However, potentials such as $V_{i}(x)=x_{1}^{2} x_{2}^{2} \ldots x_{n}^{2}-a_{i}$, with constants $a_{i}>0$ such that $\lambda_{1}^{i}>0$, satisfy conditions $\left(V_{1}\right)_{i}-\left(V_{3}\right)_{i}$ although they do not satisfy any of the conditions mentioned above. Furthermore, the following hypothesis

$$
\lim _{r \rightarrow+\infty}\left|\bigcap_{i=1,2}\left(\Omega_{M}^{V_{i}}\right) \backslash\left(B_{r}\right)\right|=0, \text { for all } M>0,
$$

is a sufficient condition for hypothesis $\left(V_{2}\right)_{i}$ (cf. [50], Theorem 1.4 and [42], Remark 1.3).

Due to hypotheses $\left(V_{1}\right)_{i}$ and $\left(V_{3}\right)_{i}$, problem $(E S)$ must be treated in the subspace of $H^{1}\left(\mathbb{R}^{N}, \mathbb{R}^{2}\right)$ defined by

$$
E:=\left\{\left(u_{1}, u_{2}\right) \in H^{1}\left(\mathbb{R}^{N}, \mathbb{R}^{2}\right): \int_{\mathbb{R}^{N}}\left(V_{1}(x) u_{1}^{2}(x)+V_{2}(x) u_{2}^{2}(x)\right) d x<+\infty\right\},
$$

which is a Hilbert space continuously embedded in $H^{1}\left(\mathbb{R}^{N}, \mathbb{R}^{2}\right)$ when endowed with the inner product

$$
\left(\left(u_{1}, u_{2}\right),\left(v_{1}, v_{2}\right)\right)_{E}:=\sum_{i=1,2} \int_{\mathbb{R}^{N}}\left(\nabla u_{i}(x) \cdot \nabla v_{i}(x)+V_{i}(x) u_{i}(x) v_{i}(x)\right) d x
$$

and the correspondent norm $\left\|\left(u_{1}, u_{2}\right)\right\|_{E}^{2}:=\left(\left(u_{1}, u_{2}\right),\left(u_{1}, u_{2}\right)\right)_{E}$ (cf. [42] and [50], both Lemma 2.1). Furthermore, $E$ is compactly embedded in the weighted spaces $L_{h(x)}^{s}\left(\mathbb{R}^{N}, \mathbb{R}^{2}\right)$, for $2 \leq s<+\infty$ if $N=2$, and for $2 \leq s<2^{\#}:=2^{*}-\frac{4}{\alpha_{0}(N-2)}$ if $N>2$ (cf. [42], Proposition 2.4 and also [50, Proposition 3.1). Hence, the spectra of operators $A_{i}$ are discrete and consist of a sequence $\lambda_{n}^{i}$ of positive eigenvalues such that $\lambda_{n}^{i} \rightarrow+\infty$ as $n \rightarrow+\infty$ (cf. [50], Lemma 3.2). Note that $2^{\#}$ is such that $2<2^{\#} \leq 2^{*}$ taking into account that $\alpha_{0}>1$. 
All hypotheses and observations made up to now, will be assumed throughout this chapter. From now both, asymptotically and super quadratic cases are treated, but separately in two sections.

\subsection{Asymptotically Quadratic Elliptic Systems}

With the aim of treating the asymptotically quadratic case, the following assumptions on the Hamiltonian $F$ are required.

$\left(F_{1}\right) \quad F(x, s, t) \in C^{1}\left(\mathbb{R}^{N} \times \mathbb{R}^{2}, \mathbb{R}^{+}\right)$and

$$
\begin{gathered}
\frac{F(x, s, t)}{h(x)}=o\left(|(s, t)|^{2}\right), \quad \text { as } \quad|(s, t)| \rightarrow 0, \\
\frac{F(x, s, t)}{|(s, t)|^{2}}=h(x)+o(1), \quad \text { as } \quad|(s, t)| \rightarrow+\infty,
\end{gathered}
$$

uniformly in $x \in \mathbb{R}^{N}$.

$\left(F_{2}\right)$ Setting $Q(x, s, t):=F_{s}(x, s, t) s+F_{t}(x, s, t) t-2 F(x, s, t)$ for all $(x, s, t) \in \mathbb{R}^{N} \times \mathbb{R}^{2}$, one has

$$
\lim _{|(s, t)| \rightarrow+\infty} Q(x, s, t)=+\infty, \quad \text { uniformly in } x \in \mathbb{R}^{N}
$$

Remark 4.2. It is worth to highlight the close relation between hypotheses $(V)$ and $\left(F_{1}\right)$. Since a linking geometry is sought, these assumptions provide the required features for the functional associated to problem $(E S)$. Thereby, note that, if instead of $F \geq 0$ as in $\left(F_{1}\right)$, it was considered $F \leq 0$, then in $(V)$ it would be asked $a_{0}>\lambda_{1}^{2}$. Indeed, in this case, problem $(E S)$ could be treated in the same way, provided that the positions of the equations were exchanged.

In view of $\left(F_{1}\right)$, given $\varepsilon>0$, for $2 \leq p<+\infty$ if $N=2$ and for $2 \leq p<2$ \# if $N>2$, there exist constants $C>0$, and $\tilde{C}_{\varepsilon}>0$ such that

$$
|F(x, u(x), v(x))| \leq C h(x)\left(\varepsilon|(u(x), v(x))|^{2}+\tilde{C}_{\varepsilon}|(u(x), v(x))|^{p}\right),
$$

almost everywhere in $\mathbb{R}^{N}$. In addition, assuming also hypotheses $\left(V_{1}\right)_{i}-\left(V_{3}\right)_{i}$ for $i=1,2$ and $(V)$, the functional

$$
\int_{\mathbb{R}^{N}}|F(x, u(x), v(x))| d x
$$

belongs to $C^{1}(E, \mathbb{R})$, since for all $\varepsilon>0$ given it satisfies

$$
\int_{\mathbb{R}^{N}}|F(x, u(x), v(x))| d x \leq \varepsilon\|(u, v)\|_{E}^{2}+C_{\varepsilon}\|(u, v)\|_{E}^{p},
$$


for some constant $C_{\varepsilon}>0$ (cf. [42], Lemma 2.2 and also [50], Lemma 3.1).

In virtue of $(V)$ and $\left(F_{1}\right)$ it is possible to assert that the growth of $F$ is controlled by the growth of $V_{i}$ by means of $h$, hence $F$ can be unbounded but under some restrictions. For instance, a function $F$ such that

$$
\left(F_{s}(x, s, t), F_{t}(x, s, t)\right)=c_{0} h(x)\left(\frac{s^{3}}{1+s^{2}}, \frac{t^{3}}{1+t^{2}}\right)
$$

or

$$
\left(F_{s}(x, s, t), F_{t}(x, s, t)\right)=c_{0} h(x)\left(\frac{s\left(s^{2}+t^{2}\right)}{1+s^{2}+t^{2}}, \frac{t\left(s^{2}+t^{2}\right)}{1+s^{2}+t^{2}}\right),
$$

where $h(x) \geq a_{0}>\lambda_{1}^{1}, \quad V_{i}(x) \geq[h(x)]^{\alpha_{0}}$ for $|x|>r_{0}$, with $c_{0}, a_{0}, r_{0}$ positive constants and $\alpha_{0}>1$, satisfy assumptions $(V)$ and $\left(F_{1}\right)$.

The main result of this section is stated below.

Theorem 4.1. Under the assumptions $\left(V_{1}\right)_{i}-\left(V_{3}\right)_{i},(V)$ and $\left(F_{1}\right)-\left(F_{2}\right)$, system 4.1) has a nontrivial solution in $H^{1}\left(\mathbb{R}^{N}, \mathbb{R}^{2}\right)$.

\subsubsection{Variational Framework}

In order to tackle problem (4.1) by means of variational methods, the strongly indefinite functional $I: E \rightarrow \mathbb{R}$ given by

$$
\begin{aligned}
I(u, v) & =\frac{1}{2} \int_{\mathbb{R}^{N}}\left(|\nabla u(x)|^{2}+V_{1}(x) u^{2}(x)-|\nabla v(x)|^{2}-V_{2}(x) v^{2}(x)\right) d x-\int_{\mathbb{R}^{N}} F(x, u(x), v(x)) d x \\
& =\frac{1}{2}\left(\|(u, 0)\|_{E}^{2}-\|(0, v)\|_{E}^{2}\right)-\int_{\mathbb{R}^{N}} F(x, u(x), v(x)) d x
\end{aligned}
$$

is associated to the elliptic system $(E S)$. Thereby, $I \in C^{1}(E, \mathbb{R})$ and

$$
\begin{aligned}
I^{\prime}(u, v)(\varphi, \psi) & =\int_{\mathbb{R}^{N}}\left(\nabla u(x) \cdot \nabla \varphi(x)+V_{1}(x) u(x) \varphi(x)\right) d x \\
& -\int_{\mathbb{R}^{N}}\left(\nabla v(x) \cdot \nabla \psi(x)+V_{2}(x) v(x) \psi(x)\right) d x \\
& -\int_{\mathbb{R}^{N}} F_{u}(x, u(x), v(x)) \varphi(x) d x-\int_{\mathbb{R}^{N}} F_{v}(x, u(x), v(x)) \psi(x) d x \\
& =((u, 0),(\varphi, 0))_{E}-((0, v),(0, \psi))_{E} \\
& -\int_{\mathbb{R}^{N}}\left(F_{u}(x, u(x), v(x)) \varphi(x)+F_{v}(x, u(x), v(x)) \psi(x)\right) d x .
\end{aligned}
$$

Hence critical points of $I$ are weak solutions for (4.1). Thus, from now on, the plan is to find a critical point to $I$ by applying Theorem 1.4 , since $I$ is an indefinite functional. 
Henceforth, it is necessary to check that $I$ satisfies $\left(I_{1}\right)-\left(I_{4}\right)$.

First, it is convenient to analyze operator $A:=\left(A_{1},-A_{2}\right)$ for $A_{i}$ defined as in $\left(V_{3}\right)_{i}$ as an operator of $L^{2}\left(\mathbb{R}^{N}, \mathbb{R}^{2}\right)$ such that $A(u, v):=\left(A_{1} u,-A_{2} v\right) \in L^{2}\left(\mathbb{R}^{N}, \mathbb{R}^{2}\right)$, for all $(u, v)$ in the domain of $A$. Moreover, for all $(u, v) \in E$, one has

$$
(A(u, v),(u, v))_{L^{2}\left(\mathbb{R}^{N}, \mathbb{R}^{2}\right)}=\|(u, 0)\|_{E}^{2}-\|(0, v)\|_{E}^{2}
$$

Hence, defining

$$
E_{1}:=\left\{(u, 0) \in H^{1}\left(\mathbb{R}^{N}, \mathbb{R}^{2}\right): \int_{\mathbb{R}^{N}} V_{1}(x) u^{2}(x) d x<+\infty\right\}
$$

and

$$
E_{2}:=\left\{(0, v) \in H^{1}\left(\mathbb{R}^{N}, \mathbb{R}^{2}\right): \int_{\mathbb{R}^{N}} V_{2}(x) v^{2}(x) d x<+\infty\right\}
$$

it yields $E:=E_{1} \oplus E_{2}$. Thus, setting $L_{1}:=I d: E_{1} \rightarrow E_{1}, L_{2}:=-I d: E_{2} \rightarrow E_{2}$ and $L: E \rightarrow E$ with $L:=L_{1}+L_{2}$, where $I d$ is identity operator, it follows that

$$
(L(u, v),(\varphi, \psi))_{E}=((u, 0),(\varphi, 0))_{E}-((0, v),(0, \psi))_{E}
$$

Therefore, $I$ satisfies $\left(I_{1}\right)$ in Theorem 1.4 since

$$
\begin{aligned}
(L(u, v),(u, v))_{E} & =((u, 0),(u, 0))_{E}-((0, v),(0, v))_{E} \\
& =\|(u, 0)\|_{E}^{2}-\|(0, v)\|_{E}^{2} \\
& =(A(u, v),(u, v))_{L^{2}\left(\mathbb{R}^{N}, \mathbb{R}^{2}\right)}
\end{aligned}
$$

and then

$$
I(u, v)=\frac{1}{2}(L(u, v),(u, v))_{E}+B(u, v)
$$

where

$$
B(u, v):=-\int_{\mathbb{R}^{N}} F(x, u(x), v(x)) d x .
$$

Remark 4.3. Since each $A_{i}$ has discrete and positive spectrum $\sigma\left(A_{i}\right)=\left\{\lambda_{n}^{i}\right\}$ for $i=1,2$, $A$ has discrete spectrum $\sigma(A)=\left\{\lambda_{n}^{1}\right\} \cup\left\{-\lambda_{n}^{2}\right\}$. In fact, since $E$ is compactly embedded in $L_{h(x)}^{s}\left(\mathbb{R}^{N}, \mathbb{R}^{2}\right)$, it implies that $\sigma(A)$ is discrete (cf. 42], Proposition 2.4). In addition, $\lambda$ is an eigenvalue of $A$ iff there exists $(u, v) \in E \backslash\{0\}$ such that $A(u, v)=\lambda(u, v)$, namely, $A_{1} u=\lambda u$ and $A_{2} v=(-\lambda) v$. Provided that, $u \neq 0$ or $v \neq 0$, then $\lambda$ is an eigenvalue of $A$ iff it is an eigenvalue of $A_{1}$ or $-\lambda$ is an eigenvalue of $A_{2}$. Therefore, $A$ has positive and negative spectra composed respectively by $\lambda_{n}^{1} \rightarrow+\infty$ and $-\lambda_{n}^{2} \rightarrow-\infty$ 
as $n \rightarrow+\infty$. Furthermore, $\lambda_{1}^{1}$ is the smallest positive eigenvalue of $A$ and $-\lambda_{1}^{2}$ is the largest negative eigenvalue of $A$, and in virtue of $\left(V_{3}\right)_{i}, 0 \notin \sigma(A)$. In addition, $E_{1}$ and $E_{2}$ correspond to the subspaces of $E$ where $A$ is positive definite, and negative definite, respectively, and both are infinite dimensional.

\subsubsection{Establishing Compactness}

So as to ensure that $I$ satisfies $\left(I_{2}\right)$ in Theorem 1.4, the following lemmas are stated and proved.

Lemma 4.1. Under the hypotheses $\left(V_{1}\right)_{i}-\left(V_{3}\right)_{i},(V)$ and $\left(F_{1}\right)$, functional $B: E \rightarrow \mathbb{R}$ defined as in (4.10) is weakly continuous.

Proof. Let $\left(u_{n}, v_{n}\right) \rightarrow(u, v)$ weakly in $E$, since $E$ is compactly embedded in $L_{h(x)}^{s}\left(\mathbb{R}^{N}, \mathbb{R}^{2}\right)$ for $2 \leq s<+\infty$ if $N=2$ and for $2 \leq s<2^{\#}$ if $N>2$, then $\left(u_{n}, v_{n}\right) \rightarrow(u, v)$, up to subsequences, strongly in $L_{h(x)}^{s}\left(\mathbb{R}^{N}, \mathbb{R}^{2}\right)$. By the converse of Lebesgue Dominated Convergence Theorem, there exist functions $\left(\varphi_{s}, \psi_{s}\right) \in L_{h(x)}^{s}\left(\mathbb{R}^{N}, \mathbb{R}^{2}\right)$ for $s=2$, and $s=p$ such that, up to subsequences, $\left|u_{n}(x)\right| \leq \varphi_{s}(x),\left|v_{n}(x)\right| \leq \psi_{s}(x)$ and $\left(u_{n}(x), v_{n}(x)\right) \rightarrow\left(\varphi_{s}(x), \psi_{s}(x)\right)$, almost everywhere in $\mathbb{R}^{N}$. Hence,

$$
F\left(x, u_{n}(x), v_{n}(x)\right) \rightarrow F(x, u(x), v(x)),
$$

almost everywhere in $\mathbb{R}^{N}$, and from 4.4 it follows that

$$
\begin{aligned}
\left|F\left(x, u_{n}(x), v_{n}(x)\right)\right| & \left.\left.\leq\left. C h(x)\left[\varepsilon \mid\left(u_{n}(x), v_{n}(x)\right)\right)\right|^{2}+\tilde{C}_{\varepsilon} \mid\left(u_{n}(x), v_{n}(x)\right)\right)\left.\right|^{p}\right] \\
& \leq \tilde{C} h(x)\left[\left|\left(\varphi_{2}(x), \psi_{2}(x)\right)\right|^{2}+\left|\left(\varphi_{p}(x), \psi_{p}(x)\right)\right|^{p}\right]
\end{aligned}
$$

almost everywhere in $\mathbb{R}^{N}$. Therefore, applying Lebesgue Dominated Convergence Theorem, it yields

$$
\int_{\mathbb{R}^{N}} F\left(x, u_{n}(x), v_{n}(x)\right) d x \rightarrow \int_{\mathbb{R}^{N}} F(x, u(x), v(x)) d x,
$$

as $n \rightarrow+\infty$, namely, $B\left(u_{n}, v_{n}\right) \rightarrow B(u, v)$ and $B$ is weakly continuous.

Lemma 4.2. Assuming that $\left(V_{1}\right)_{i}-\left(V_{3}\right)_{i},(V)$ and $\left(F_{1}\right)$ hold, functional $I$ is uniformly differentiable on bounded sets of $E$.

Proof. Fixed $R>0$ and given $\left(u_{1}, u_{2}\right)+\left(v_{1}, v_{2}\right),\left(v_{1}, v_{2}\right) \in B_{R} \subset E$, the closed ball with 
radius $R$, centered on the origin, one has

$$
\begin{aligned}
& \left|B\left(u_{1}+v_{1}, u_{2}+v_{2}\right)-B\left(u_{1}, u_{2}\right)-B^{\prime}\left(u_{1}, u_{2}\right)\left(v_{1}, v_{2}\right)\right| \\
= & \int_{\mathbb{R}^{N}}\left|F\left(x, u_{1}+v_{1}, u_{2}+v_{2}\right)-F\left(x, u_{1}, u_{2}\right)-F_{u}\left(x, u_{1}, u_{2}\right) v_{1}-F_{v}\left(x, u_{1}, u_{2}\right) v_{2}\right| d x \\
\leq & \int_{\mathbb{R}^{N}} \frac{1}{h(x)^{\frac{1}{2}}}\left|F_{u}\left(x, z_{1}, z_{2}\right)+F_{v}\left(x, z_{1}, z_{2}\right)-F_{u}\left(x, u_{1}, u_{2}\right)-F_{v}\left(x, u_{1}, u_{2}\right)\right|\left|h(x)^{\frac{1}{2}}\left(v_{1}, v_{2}\right)\right| d x \\
\leq & C_{2}\|\xi\|_{L^{2}\left(\mathbb{R}^{N}\right)}\left\|\left(v_{1}, v_{2}\right)\right\|_{E},
\end{aligned}
$$

where

$\xi(x):=\frac{1}{h(x)^{\frac{1}{2}}}\left|F_{u}\left(x, z_{1}(x), z_{2}(x)\right)+F_{v}\left(x, z_{1}(x), z_{2}(x)\right)-F_{u}\left(x, u_{1}(x), u_{2}(x)\right)-F_{v}\left(x, u_{1}(x), u_{2}(x)\right)\right|$

and $\left(z_{1}(x), z_{2}(x)\right)=\left(u_{1}(x), u_{2}(x)\right)+\left(\theta_{1}(x) v_{1}(x), \theta_{2}(x) v_{2}(x)\right)$, where $0 \leq \theta_{i}(x) \leq 1$ is given by Mean Value Theorem and $C_{2}>0$ is the constant given by the continuous embedding $E \hookrightarrow L_{h(x)}^{2}\left(\mathbb{R}^{N}, \mathbb{R}^{2}\right)$. In order to prove that $B$ is uniformly differentiable on bounded sets of $E$, it is sufficient to show that given $\varepsilon>0$ there exist $\delta>0$ such that $C_{2}\|\xi\|_{L^{2}\left(\mathbb{R}^{N}\right)} \leq \varepsilon$ for all $\left(u_{1}, u_{2}\right)+\left(v_{1}, v_{2}\right),\left(v_{1}, v_{2}\right) \in B_{R} \subset E$ with $\left\|\left(v_{1}, v_{2}\right)\right\|_{E} \leq \delta$. However, by definition of limit, this is equivalent to show that $\xi \rightarrow 0$ in $L^{2}\left(\mathbb{R}^{N}\right)$ as $\left(v_{1}, v_{2}\right) \rightarrow 0$ in $E$.

Observe that $\left(v_{1}, v_{2}\right) \rightarrow 0$ in $E$ implies $\left(v_{1}, v_{2}\right) \rightarrow 0$ in $L_{h(x)}^{s}\left(\mathbb{R}^{N}, \mathbb{R}^{2}\right)$ for $2 \leq s<+\infty$ if $N=2$ and for $2 \leq s<2^{\#}$ if $N>2$, then $\left(v_{1}(x), v_{2}(x)\right) \rightarrow 0$ almost everywhere in $\mathbb{R}^{N}$ and there exist $\left(\varphi_{s}, \psi_{s}\right) \in L_{h(x)}^{s}\left(\mathbb{R}^{N}, \mathbb{R}^{2}\right)$ such that $\left|v_{1}(x)\right| \leq \varphi_{s}(x)$, $\left|v_{2}(x)\right| \leq \psi_{s}(x)$ almost everywhere in $\mathbb{R}^{N}$. Moreover, $\left(z_{1}(x), z_{2}(x)\right) \rightarrow\left(u_{1}(x), u_{2}(x)\right)$ almost everywhere in $\mathbb{R}^{N}$. Thus, $\xi(x) \rightarrow 0$ almost everywhere in $\mathbb{R}^{N}$. Furthermore, from $\left(F_{1}\right)$ and L'Hospital rule, one has

$\left|F_{u}\left(x, z_{1}(x), z_{2}(x)\right)\right| \leq C h(x)\left|\left(z_{1}(x), z_{2}(x)\right)\right| \quad$ and $\quad\left|F_{v}\left(x, z_{1}(x), z_{2}(x)\right)\right| \leq C h(x)\left|\left(z_{1}(x), z_{2}(x)\right)\right|$,

almost everywhere in $\mathbb{R}^{N}$, for some constant $C>0$ and the analogous holds for $u_{i}$ instead of $z_{i}$. Then, for $s=2$ it follows that

$$
\begin{aligned}
|\xi(x)|^{2} & \leq \frac{2}{h(x)}\left[\left|F_{u}\left(x, z_{1}(x), z_{2}(x)\right)\right|+\left|F_{v}\left(x, z_{1}(x), z_{2}(x)\right)\right|\right]^{2} \\
& +\frac{2}{h(x)}\left[\left|F_{u}\left(x, u_{1}(x), u_{2}(x)\right)\right|+\left|F_{v}\left(x, u_{1}(x), u_{2}(x)\right)\right|\right]^{2} \\
& \leq \frac{2}{h(x)}\left[\left(2 C h(x)\left|\left(z_{1}(x), z_{2}(x)\right)\right|\right)^{2}+\left(2 C h(x)\left|\left(u_{1}(x), u_{2}(x)\right)\right|\right)^{2}\right] \\
& \leq \frac{8 C^{2} h(x)^{2}}{h(x)}\left[\left|\left(v_{1}(x), v_{2}(x)\right)\right|^{2}+3\left|\left(u_{1}(x), u_{2}(x)\right)\right|^{2}\right] \\
& \leq 24 C^{2}\left[h(x)\left|\left(\varphi_{2}(x), \psi_{2}(x)\right)\right|^{2}+h(x)\left|\left(u_{1}(x), u_{2}(x)\right)\right|^{2}\right] .
\end{aligned}
$$


Therefore, by applying Lebesgue Dominated Convergence Theorem it yields

$$
\|\xi\|_{L^{2}\left(\mathbb{R}^{N}\right)}^{2}=\int_{\mathbb{R}^{N}}|\xi(x)|^{2} d x \rightarrow 0
$$

as $\left(v_{1}, v_{2}\right) \rightarrow 0$ in $E$ and the result hold.

\subsubsection{Linking Geometry}

Under the purpose of proving that $I$ satisfies $\left(I_{3}\right)$ in Theorem 1.4 as usual, set

$$
S:=\partial B_{\rho} \cap E_{1} \quad \text { and } \quad Q:=\left\{(r e, v): r \geq 0,(0, v) \in E_{2},\|(r e, v)\|_{E} \leq r_{1}\right\}
$$

where $0<\rho<r_{1}$ are constants, $(e, 0) \in E_{1}$ and $e$ is an eigenfunction associated to $\lambda_{1}^{1}$ the first eigenvalue of $A_{1}$, with $\|(e, 0)\|_{E}=1$, therefore, such $S$ and $Q$ "link". Due to the strict inequality $a_{0}>\lambda_{1}^{1}$ in hypothesis $(V)$ and from 4.8$)$, such an $e$ satisfies

$$
\begin{aligned}
1 & =\|(e, 0)\|_{E}^{2}=(A(e, 0),(e, 0))_{L^{2}\left(\mathbb{R}^{N}, \mathbb{R}^{2}\right)} \\
& =\int_{\mathbb{R}^{N}}\left(|\nabla e(x)|^{2}+V_{1}(x) e^{2}(x)\right) d x=\left(A_{i} e, e\right)_{L^{2}\left(\mathbb{R}^{N}\right)} \\
& =\lambda_{1}^{1}\|e\|_{L^{2}\left(\mathbb{R}^{N}\right)}^{2} \\
& <a_{0}\|(e, 0)\|_{L^{2}\left(\mathbb{R}^{N}, \mathbb{R}^{2}\right)} \\
& \leq \int_{\mathbb{R}^{N}} h(x)|(e(x), 0)|^{2} d x
\end{aligned}
$$

Thereby, by means of 4.14 it is possible to show that if $r_{1}>0$ is large enough, then $\left.I\right|_{S} \geq \alpha>0$ and $\left.I\right|_{\partial Q} \leq 0$ for some $\alpha>0$. Thus, $I$ satisfies $\left(I_{3}\right)$ for some $\alpha>0, \omega=0$ and arbitrary $(0, v) \in E_{2}$. Indeed, next lemma gives the result.

Lemma 4.3. Suppose that $\left(V_{1}\right)_{i}-\left(V_{3}\right)_{i},(V)$ and $\left(F_{1}\right)$ hold, then I satisfies $\left(I_{3}\right)$.

Proof. Note that $S \subset E_{1}$, then from (4.6) and (4.5), for all $\left(u_{1}, 0\right) \in S$, it yields

$$
\begin{aligned}
I\left(u_{1}, 0\right) & =\frac{1}{2}\left\|\left(u_{1}, 0\right)\right\|_{E}^{2}-\int_{\mathbb{R}^{N}} F\left(x, u_{1}(x), 0\right) d x \\
& \geq \frac{1}{2} \rho^{2}-\left(\varepsilon\left\|\left(u_{1}, 0\right)\right\|_{E}^{2}+C_{\varepsilon}\left\|\left(u_{1}, 0\right)\right\|_{E}^{p}\right) \\
& =\rho^{2}\left[\left(\frac{1}{2}-\varepsilon\right)-C_{\varepsilon} \rho^{p-2}\right] \\
& \geq \rho^{2}\left(d_{1}-d_{2}\right)=\alpha>0,
\end{aligned}
$$


where $\varepsilon, \rho$ are chosen small enough, so that $1>2 \varepsilon$ and

$$
d_{1}:=\frac{1}{2}-\varepsilon>C_{\varepsilon} \rho^{p-2}=: d_{2}
$$

Therefore, from 4.15), $\left(I_{3}\right)(i)$ holds for $I$.

In order to prove that $I$ satisfies $\left(I_{3}\right)(i i)$ in Theorem 1.4 , with $\omega=0$, observe that $I(0, v) \leq 0$, for all $(0, v) \in E_{2}$, then it suffices to show that $I(r e, v) \leq 0$ for $r>0,(0, v) \in E_{2}$ and $\|(r e, v)\|_{E} \geq r_{1}$, for some $r_{1}>0$ large enough. Arguing indirectly assume that some sequence $\left(r_{n} e, v_{n}\right) \subset \mathbb{R}^{+} e \oplus E_{2}$ satisfies $\left\|\left(r_{n} e, v_{n}\right)\right\|_{E} \rightarrow+\infty$ and $I\left(r_{n} e, v_{n}\right)>0$ for all $n \in \mathbb{N}$. Seeking a contradiction, set

$$
\left(s_{n} e, w_{n}\right):=\frac{\left(r_{n} e, v_{n}\right)}{\left\|\left(r_{n} e, v_{n}\right)\right\|_{E}},
$$

where $s_{n} \in \mathbb{R}^{+},\left(0, w_{n}\right) \in E_{2}$ and $\left\|\left(s_{n} e, w_{n}\right)\right\|_{E}=1$. Provided that $\left(s_{n} e, w_{n}\right)$ is bounded, up to subsequences, it follows that $\left(s_{n} e, w_{n}\right) \rightarrow(s e, w)$ in $E$, hence $\left(s_{n} e, w_{n}\right) \rightarrow(s e, w)$ in $L_{h(x)}^{2}\left(\mathbb{R}^{N}, \mathbb{R}^{2}\right)$, due to the compact embedding $E \hookrightarrow L_{h(x)}^{2}\left(\mathbb{R}^{N}, \mathbb{R}^{2}\right)$. Then, up to subsequences, $\left(s_{n} e(x), w_{n}(x)\right) \rightarrow(s e(x), w(x))$ almost everywhere in $\mathbb{R}^{N}, s_{n} \rightarrow s$ in $\mathbb{R}^{+}$and $\left(0, w_{n}\right) \rightarrow(0, w)$ in $E_{2}$, since $s_{n}$ and $\left(0, w_{n}\right)$ are also bounded. Noting that

$$
1=\left\|\left(s_{n} e, w_{n}\right)\right\|_{E}^{2}=s_{n}^{2}+\left\|\left(0, w_{n}\right)\right\|_{E}^{2}
$$

it follows that $0 \leq s_{n}^{2} \leq 1$, and it yields

$$
\begin{aligned}
\frac{I\left(r_{n} e, v_{n}\right)}{\left\|\left(r_{n} e, v_{n}\right)\right\|_{E}^{2}} & =\frac{1}{2}\left(s_{n}^{2}\|(e, 0)\|_{E}^{2}-\left\|\left(0, w_{n}\right)\right\|_{E}^{2}\right)-\int_{\mathbb{R}^{N}} \frac{F\left(x, r_{n} e(x), v_{n}(x)\right)}{\left\|\left(r_{n} e, v_{n}\right)\right\|_{E}^{2}} d x \\
& =s_{n}^{2}-\frac{1}{2}-\int_{\mathbb{R}^{N}} \frac{F\left(x, r_{n} e(x), v_{n}(x)\right)}{\left\|\left(r_{n} e, v_{n}\right)\right\|_{E}^{2}} d x>0
\end{aligned}
$$

hence $\frac{1}{2} \leq s^{2} \leq 1$. Moreover, from 4.14 one has $1<\int_{\mathbb{R}^{N}} h(x)|(e(x), 0)|^{2} d x$. Then,

$$
\begin{aligned}
0 & >s^{2}-s^{2} \int_{\mathbb{R}^{N}} h(x)|(e(x), 0)|^{2} d x \\
& >s^{2}\left(1-\int_{\mathbb{R}^{N}} h(x)|(e(x), 0)|^{2} d x\right)-\frac{1}{2}-\int_{\mathbb{R}^{N}} h(x)|(0, w(x))|^{2} d x .
\end{aligned}
$$

On the other hand, since $\left(s_{n} e, v_{n}\right)$ is convergent in $L_{h(x)}^{2}\left(\mathbb{R}^{N}, \mathbb{R}^{2}\right)$, there exists some $\left(\varphi_{2}, \psi_{2}\right) \in L_{h(x)}^{2}\left(\mathbb{R}^{N}, \mathbb{R}^{2}\right)$ such that $\left|s_{n} e(x)\right| \leq \varphi_{2}(x)$ and $\left|w_{n}(x)\right| \leq \psi_{2}(x)$ almost ev- 
erywhere in $\mathbb{R}^{N}$ and from 4.4 with $p=2$, it follows that

$$
\left|\frac{F\left(x, r_{n} e(x), v_{n}(x)\right)}{\|\left.\left(r_{n} e, v_{n}\right)\right|_{E} ^{2}}\right| \leq \tilde{C} h(x)\left|\left(s_{n} e(x), w_{n}(x)\right)\right|^{2} \leq \tilde{C} h(x)\left|\left(\varphi_{2}(x), \psi_{2}(x)\right)\right|^{2},
$$

almost everywhere in $\mathbb{R}^{N}$, for some constant $\tilde{C}>0$. Provided that $\left\|\left(r_{n} e, v_{n}\right)\right\|_{E} \rightarrow+\infty$, and $\left(s_{n} e(x), w_{n}(x)\right) \rightarrow(s e(x), w(x)) \neq 0$, almost everywhere in $\operatorname{supp}(e)$, it follows that $\left(r_{n} e(x), v_{n}(x)\right)=\left(s_{n} e(x), w_{n}(x)\right)\left\|\left(r_{n} e, v_{n}\right)\right\|_{E} \rightarrow+\infty$ almost everywhere in $\operatorname{supp}(e)$, as $n \rightarrow+\infty$, hence in view of $\left(F_{1}\right)$ one has

$$
\begin{aligned}
\frac{F\left(x, r_{n} e(x), v_{n}(x)\right)}{\left\|\left(r_{n} e, v_{n}\right)\right\|_{E}^{2}} & =\frac{F\left(x,\left\|r_{n} e+u_{n}\right\|_{E}\left(s_{n} e(x), w_{n}(x)\right)\left|\left(s_{n} e(x), w_{n}(x)\right)\right|^{2}\right.}{\left\|r_{n} e+u_{n}\right\|_{E}^{2}\left|\left(s_{n} e(x), w_{n}(x)\right)\right|^{2}} \\
& =h(x)|(\operatorname{se}(x), w(x))|^{2}+o_{n}(1),
\end{aligned}
$$

almost everywhere in $\operatorname{supp}(s e, w)$ as $n \rightarrow+\infty$. Note that, $\operatorname{supp}(s e, w) \neq \emptyset$, because $\operatorname{supp}(e) \neq \emptyset$. Thus, by Lebesgue Dominated Convergence Theorem,

$$
\int_{\mathbb{R}^{N}} \frac{F\left(x, r_{n} e(x), v_{n}(x)\right)}{\left\|\left(r_{n} e, v_{n}\right)\right\|_{E}^{2}} d x \rightarrow \int_{\mathbb{R}^{N}} h(x)|(\operatorname{se}(x), w(x))|^{2} d x,
$$

as $n \rightarrow+\infty$. From 4.16 one has

$$
s_{n}^{2}-\frac{1}{2}-\int_{\mathbb{R}^{N}} \frac{F\left(x, r_{n} e(x), v_{n}(x)\right)}{\left\|\left(r_{n} e, v_{n}\right)\right\|_{E}^{2}} d x>0 .
$$

Passing to the limit as $n \rightarrow+\infty$, it yields

$$
\begin{aligned}
0 & \leq s^{2}-\frac{1}{2}-\int_{\mathbb{R}^{N}} h(x)|(s e(x), w(x))|^{2} d x \\
& =s^{2}\left(1-\int_{\Omega} h(x)|(e(x), 0)|^{2} d x\right)-\frac{1}{2}-\int_{\Omega} h(x)|(0, w(x))|^{2} d x
\end{aligned}
$$

which is contrary to 4.17). Therefore the result holds.

\subsubsection{Boundedness of Cerami Sequences}

Next lemma ensures $I$ satisfies last hypothesis in Theorem 1.4. Finally, with this result it will be possible to prove Theorem 4.1 .

Lemma 4.4. Assuming that $\left(V_{1}\right)_{i}-\left(V_{3}\right)_{i},(V)$ and $\left(F_{1}\right)-\left(F_{2}\right)$ hold, I satisfies $\left(I_{4}\right)$. 
Proof. Let $b>0$ be an arbitrary constant, and take $\left(u_{n}, v_{n}\right) \subset I^{-1}([c-b, c+b])$ such that $\left(1+\left\|\left(u_{n}, v_{n}\right)\right\|_{E}\right)\left\|I^{\prime}\left(u_{n}, v_{n}\right)\right\|_{E^{\prime}} \rightarrow 0$, it is necessary to show that $\left(u_{n}, v_{n}\right)$ is bounded. Suppose by contradiction that $\left\|\left(u_{n}, v_{n}\right)\right\|_{E} \rightarrow+\infty$, up to subsequences. Setting $\left(\tilde{u}_{n}, \tilde{v}_{n}\right):=\frac{\left(u_{n}, v_{n}\right)}{\left\|\left(u_{n}, v_{n}\right)\right\|_{E}}$, it is bounded, hence $\left(\tilde{u}_{n}, \tilde{v}_{n}\right) \rightarrow(\tilde{u}, \tilde{v})$ in $E$ and due to the compact embeddings $E \hookrightarrow L_{h(x)}^{s}\left(\mathbb{R}^{N}, \mathbb{R}^{2}\right)$ for $2 \leq s<+\infty$ if $N=2$ and $2 \leq s<2$ \# if $N>2$, it implies that $\left(\tilde{u}_{n}, \tilde{v}_{n}\right) \rightarrow(\tilde{u}, \tilde{v})$ in $L_{h(x)}^{s}\left(\mathbb{R}^{N}, \mathbb{R}^{2}\right)$. Writing $\left(u_{n}, v_{n}\right)=\left(u_{n}, 0\right)+\left(0, v_{n}\right) \in$ $E_{1} \oplus E_{2}$, it satisfies

$$
\begin{aligned}
o_{n}(1) & =\frac{1}{\left\|\left(u_{n}, v_{n}\right)\right\|_{E}} I^{\prime}\left(u_{n}, v_{n}\right)\left(\tilde{u}_{n}, 0\right) \\
& =\left\|\left(\tilde{u}_{n}, 0\right)\right\|_{E}^{2}-\int_{\mathbb{R}^{N}} \frac{F_{u}\left(x, u_{n}(x), v_{n}(x)\right)}{\left\|\left(u_{n}, v_{n}\right)\right\|_{E}} \tilde{u}_{n}(x) d x \\
& =\left\|\left(\tilde{u}_{n}, 0\right)\right\|_{E}^{2}-\int_{\mathbb{R}^{N}} \frac{F_{u}\left(x, u_{n}(x), v_{n}(x)\right)}{u_{n}(x)} \tilde{u}_{n}^{2}(x) d x
\end{aligned}
$$

and

$$
\begin{aligned}
o_{n}(1) & =\frac{1}{\left\|\left(u_{n}, v_{n}\right)\right\|_{E}} I^{\prime}\left(u_{n}, v_{n}\right)\left(0, \tilde{v}_{n}\right) \\
& =-\left\|\left(0, \tilde{v}_{n}\right)\right\|_{E}^{2}-\int_{\mathbb{R}^{N}} \frac{F_{v}\left(x, u_{n}(x), v_{n}(x)\right)}{v_{n}(x)} \tilde{v}_{n}^{2}(x) d x .
\end{aligned}
$$

Subtracting 4.20 from 4.19, and using that $1=\left\|\left(\tilde{u}_{n}, 0\right)\right\|_{E}^{2}+\left\|\left(0, \tilde{v}_{n}\right)\right\|_{E}^{2}$, it yields

$$
o_{n}(1)=1-\int_{\mathbb{R}^{N}}\left[\frac{F_{u}\left(x, u_{n}(x), v_{n}(x)\right)}{u_{n}(x)} \tilde{u}_{n}^{2}(x)-\frac{F_{v}\left(x, u_{n}(x), v_{n}(x)\right)}{v_{n}(x)} \tilde{v}_{n}^{2}(x)\right] d x .
$$

Provided that $\left(\tilde{u}_{n}, \tilde{v}_{n}\right) \rightarrow(\tilde{u}, \tilde{v})$ in $L_{h(x)}^{2}\left(\mathbb{R}^{N}, \mathbb{R}^{2}\right)$, there exist $\left(\varphi_{2}, \psi_{2}\right) \in L_{h(x)}^{2}\left(\mathbb{R}^{N}, \mathbb{R}^{2}\right)$ such that $\left|\tilde{u}_{n}(x)\right| \leq \varphi_{2}(x)$ and $\left|\tilde{v}_{n}(x)\right| \leq \psi_{2}(x)$, almost everywhere in $\mathbb{R}^{N}$. Furthermore, from $\left(F_{1}\right)$ it follows that

$$
\left|\frac{F_{u}\left(x, u_{n}(x), v_{n}(x)\right)}{u_{n}(x)}\right| \leq C h(x) \text { and }\left|\frac{F_{v}\left(x, u_{n}(x), v_{n}(x)\right)}{v_{n}(x)}\right| \leq C h(x),
$$

for some constant $C>0$, almost everywhere in $\mathbb{R}^{N}$. Hence,

$$
\begin{aligned}
\left|\frac{F_{u}\left(x, u_{n}(x), v_{n}(x)\right)}{u_{n}(x)} \tilde{u}_{n}^{2}(x)-\frac{F_{v}\left(x, u_{n}(x), v_{n}(x)\right)}{v_{n}(x)} \tilde{v}_{n}^{2}(x)\right| & \leq C h(x)\left(\tilde{u}_{n}^{2}(x)+\tilde{v}_{n}^{2}(x)\right) \\
& \leq C h(x)\left(\varphi_{2}^{2}(x)+\psi_{2}^{2}(x)\right) .
\end{aligned}
$$


Since $\left(\tilde{u}_{n}, \tilde{v}_{n}\right) \rightarrow(\tilde{u}, \tilde{v})$ in $L_{h(x)}^{2}\left(\mathbb{R}^{N}, \mathbb{R}^{2}\right),\left|\left(u_{n}(x), v_{n}(x)\right)\right| \rightarrow+\infty$, for all $x \in \mathbb{R}^{N}$ such that $(\tilde{u}(x), \tilde{v}(x)) \neq 0$, then from $\left(F_{1}\right)$ and L'Hospital rule, it follows that

$$
\frac{F_{u}\left(x, u_{n}(x), v_{n}(x)\right)}{u_{n}(x)} \tilde{u}_{n}^{2}(x)-\frac{F_{v}\left(x, u_{n}(x), v_{n}(x)\right)}{v_{n}(x)} \tilde{v}_{n}^{2}(x) \rightarrow 2 h(x)\left[\tilde{u}^{2}(x)-\tilde{v}^{2}(x)\right],
$$

as $n \rightarrow+\infty$, for all $x \in \mathbb{R}^{N}(\tilde{u}(x), \tilde{v}(x)) \neq 0$. Therefore, by Lebesgue Dominated Convergence Theorem one has

$$
\int_{\mathbb{R}^{N}}\left[\frac{F_{u}\left(x, u_{n}(x), v_{n}(x)\right)}{u_{n}(x)} \tilde{u}_{n}^{2}(x)-\frac{F_{v}\left(x, u_{n}(x), v_{n}(x)\right)}{v_{n}(x)} \tilde{v}_{n}^{2}(x)\right] d x \rightarrow \int_{\mathbb{R}^{N}} 2 h(x)\left[\tilde{u}^{2}(x)-\tilde{v}^{2}(x)\right] d x .
$$

Hence, passing to the limit in 4.21 as $n \rightarrow+\infty$, it yields

$$
\int_{\mathbb{R}^{N}} 2 h(x)\left[\tilde{u}^{2}(x)-\tilde{v}^{2}(x)\right] d x=1
$$

which implies there exists $\Omega \subset \mathbb{R}^{N}$ such that $|\Omega|>0$ and $(\tilde{u}(x), \tilde{v}(x)) \neq 0$ for all $x \in \Omega$, otherwise 4.23 yields a contradiction.

On the other hand, since $\left(u_{n}, v_{n}\right)$ is a Cerami sequence, there exists a constant $M_{0}>0$ such that

$$
\begin{aligned}
M_{0} & \geq I\left(u_{n}, v_{n}\right)-\frac{1}{2} I^{\prime}\left(u_{n}, v_{n}\right)\left(u_{n}, v_{n}\right) \\
& =\frac{1}{2} \int_{\mathbb{R}^{N}}\left(F_{u}\left(x, u_{n}, v_{n}\right) u_{n}-F_{v}\left(x, u_{n}, v_{n}\right) v_{n}-2 F\left(x, u_{n}, v_{n}\right)\right) d x \\
& =\frac{1}{2} \int_{\mathbb{R}^{N}} Q\left(x, u_{n}(x), v_{n}(x)\right) d x \\
& \geq \frac{1}{2} \int_{\Omega} Q\left(x, u_{n}(x), v_{n}(x)\right) d x
\end{aligned}
$$

Provided that $\left|\left(u_{n}(x), v_{n}(x)\right)\right| \rightarrow+\infty$, for all $x \in \Omega$, in view of $\left(F_{2}\right)$, it yields

$$
\frac{1}{2} \int_{\Omega} Q\left(x, u_{n}(x), v_{n}(x)\right) d x \rightarrow+\infty
$$

as $n \rightarrow+\infty$. Passing 4.24 to the limit, a contradiction holds.

At long last, the main result is proved.

Proof of Theorem 4.1. Provided that $I$ satisfies all assumptions $\left(I_{1}\right)-\left(I_{4}\right)$ in Theorem 1.4, it ensures a critical point $(u, v) \in E$ of $I$, with $I(u, v)=c \geq \alpha>0$. It implies that $I^{\prime}(u, v)(\varphi, \psi)=0$, for all $(\varphi, \psi) \in E$ and $I(u, v)>I(0,0)$, thus $(u, v) \neq 0$. Since $I \in C^{1}(E, \mathbb{R})$, it yields that $(u, v)$ is a nontrivial solution to system $(E S)$ in 4.1 . 


\subsection{Super Quadratic Elliptic Systems}

The following assumptions on the Hamiltonian $F$ are required for the super quadratic case.

$$
\left(F_{1}^{\prime}\right) \quad F(x, s, t) \in C^{1}\left(\mathbb{R}^{N} \times \mathbb{R}^{2}, \mathbb{R}^{+}\right)
$$

$$
\frac{\left|\left(F_{s}(x, s, t), F_{t}(x, s, t)\right)\right|}{h(x)}=o(|(s, t)|) \quad \text { as } \quad|(s, t)| \rightarrow 0 \text {, uniformly in } x \text {, }
$$

and for some $2<p<+\infty$ if $N=2$ or $2<p<2^{\#}:=\frac{4}{\alpha_{0}(N-2)}$ if $N>2$,

$$
\left|\left(F_{s}(x, s, t), F_{t}(x, s, t)\right)\right| \leq c_{0} h(x)\left(1+|(s, t)|^{p-1}\right), \quad \text { for } \quad \text { all } \quad(x, s, t) \in \mathbb{R}^{N} \times \mathbb{R}^{2}
$$

$\left(F_{2}^{\prime}\right) \lim _{|(s, t)| \rightarrow+\infty} \frac{F(x, s, t)}{|(s, t)|^{2}}=+\infty$, uniformly in $x$.

Note that for such a $p$ given in $\left(F_{1}^{\prime}\right)$ and arbitrary $\varepsilon>0$, there exist constants $C>0, \tilde{C}_{\varepsilon}>0$ such that 4.4 and 4.5 hold. Hence, the functional

$$
\int_{\mathbb{R}^{N}}|F(x, u(x), v(x))| d x
$$

belongs to $C^{1}(E, \mathbb{R})$ (cf. [42], Lemma 2.2 and also [50], Lemma 3.1).

In addition, defining $Q(x, u, v)=F_{u}(x, u, v) u+F_{v}(x, u, v) v-2 F(x, u, v)$ as in $\left(F_{2}\right)$, in order to ensure the boundedness of Cerami sequences, one of the following conditions is required.

$\left(F_{3}^{\prime}\right)$ There exist constants $c_{1}>0$ and $\theta>\min \left\{1, \frac{N}{2}(p-2)\right\}$ such that for all $(x, u, v) \in \mathbb{R}^{N} \times \mathbb{R}^{2}$,

$$
Q(x, u, v) \geq c_{1} h(x)|(u, v)|^{\theta}
$$

or

$\left(F_{3}^{\prime \prime}\right)$ There exists a constant $D \geq 1$ such that

$$
Q(x, w, z) \leq D Q(x, u, v)
$$

for all $(u, v),(w, z) \in \mathbb{R}^{2}$ with $|(w, z)| \leq|(u, v)|$.

Remark 4.4. It is worth to mention that since the functional associated to system $(E S)$ 
is indefinite, hypothesis $\left(F_{3}^{\prime}\right)$ is relevant to establish the boundedness of Cerami sequences. Indeed, $\left(F_{3}^{\prime}\right)$ is a consequence of both following conditions $(A R)$ and $\left(F_{4}^{\prime}\right)$, which also give the boundedness of Cerami sequences.

(AR) There exists $\mu>2$ such that for all $(x, u, v) \in \mathbb{R}^{N} \times \mathbb{R}^{2}$,

$$
0 \leq \mu F(x, u, v) \leq F_{u}(x, u, v) u+F_{v}(x, u, v) v
$$

$\left(F_{4}^{\prime}\right) \quad$ There exist constants $c_{1}>0$ and $\theta>\min \left\{1, \frac{N}{2}(p-2)\right\}$ such that for all $(x, u, v) \in \mathbb{R}^{N} \times \mathbb{R}^{2}$,

$$
F(x, u, v) \geq c_{1} h(x)|u, v|^{\theta} .
$$

Note that from $(A R)$ and $\left(F_{4}^{\prime}\right)$ for all $(x, u, v) \in \mathbb{R}^{N} \times \mathbb{R}^{2}$, one has

$$
F_{u}(x, u, v) u+F_{v}(x, u, v) v-2 F(x, u, v) \geq(\mu-2) F(x, u, v) \geq(\mu-2) c_{1} h(x)|u, v|^{\theta},
$$

showing that $(A R)$ and $\left(F_{4}^{\prime}\right)$ imply $\left(F_{3}^{\prime}\right)$. Moreover, only hypothesis $(A R)$ is not sufficient for the boundedness, provided that standard arguments involving this assumption only bounds the difference $\left\|\left(u_{n}, 0\right)\right\|_{E}^{2}-\left\|\left(0, v_{n}\right)\right\|_{E}^{2}$, which appears in the functional. Furthermore, considering $\theta>\frac{N}{2} \frac{\alpha_{0}}{\alpha_{0}-1}(p-2)$ instead of $\theta>\frac{N}{2}(p-2)$ and exploiting the hypotheses assumed on $V_{i}$, it is possible to weak hypothesis $\left(F_{3}^{\prime}\right)$ only assuming

$$
F_{u}(x, u, v) u+F_{v}(x, u, v) v-2 F(x, u, v) \geq c_{1}|(u, v)|^{\theta} .
$$

Remark 4.5. Hypothesis $\left(F_{3}^{\prime \prime}\right)$ was introduced by Jeanjean and Tanaka [29], so as to bound Cerami sequences in cases where conditions as $(A R)$ or $\left(F_{4}^{\prime}\right)$ are not satisfied by the nonlinearity, for example when $F(x, s, t)=c_{0} h(x)(\log |(s, t)|)^{\frac{1}{2}}\left(s^{2}, t^{2}\right)$, for $|(s, t)| \geq 1$.

Similarly to asymptotically quadratic case, the relation between $(V)$ and $\left(F_{1}^{\prime}\right)$ shows that the growth of $F$ is controlled by the growth of $V_{i}$ by means of $h$, hence $F$ can be unbounded but under some restrictions. For instance, a function $F$ such that

$$
\left(F_{s}(x, s, t), F_{t}(x, s, t)\right)=c_{0} h(x)\left(|(s, t)|^{p-2} s,|(s, t)|^{p-2} t\right)
$$

where $h(x) \geq a_{0}>\lambda_{1}^{1}, \quad V_{i}(x) \geq[h(x)]^{\alpha_{0}}$ for $|x|>r_{0}$, with $c_{0}, a_{0}, r_{0}$ positive constants and $\alpha_{0}>1$, satisfy assumptions $(V)$ and $\left(F_{1}\right)$.

Now, the main result of this section is stated. 
Theorem 4.2. Suppose that $\left(V_{1}\right)_{i}-\left(V_{3}\right)_{i},(V)$ and $\left(F_{1}^{\prime}\right)-\left(F_{3}^{\prime}\right)$ hold, then system (4.1) has a nontrivial solution in $H^{1}\left(\mathbb{R}^{N}, \mathbb{R}^{2}\right)$. If $\left(F_{3}^{\prime}\right)$ is replaced by $\left(F_{3}^{\prime \prime}\right)$, the conclusion still remains true.

Under the purpose of proving Theorem 4.2, the variational structure established in Subsection 4.1.1 is assumed here. Thereby, $I$ satisfies $\left(I_{1}\right)$ in Theorem 1.4, which must be applied with the aim of getting a non trivial critical point to $I$. Furthermore, the conclusions of Lemmas 4.1 and 4.2, in Subsection 4.1.2, are exactly the same replacing $\left(F_{1}\right)$ by $\left(F_{1}^{\prime}\right)$. Thus, $\left(I_{2}\right)$ is satisfied by $I$ as well.

\subsubsection{Linking Structure}

In order to prove that $I$ satisfies $\left(I_{3}\right)$ in Theorem 1.4, all arguments in Subsection 4.1.3 are adapted to super quadratic case. As before,

$$
S:=\left(\partial B_{\rho} \cap E_{1}\right) \quad \text { and } \quad Q:=\left\{(r e, v): r \geq 0,(0, v) \in E_{2},\|(r e, v)\|_{E} \leq r_{1}\right\}
$$

where $0<\rho<r_{1}$ are constants and $(e, 0) \in E_{1}$ is an arbitrary vector with $\|(e, 0)\|_{E}=1$, therefore, such $S$ and $Q$ "link". Next lemma shows that if $r_{1}>0$ is large enough, then $\left.I\right|_{S} \geq \alpha>0$ and $\left.I\right|_{\partial Q} \leq 0$ for some $\alpha>0$. Thus, $I$ satisfies $\left(I_{3}\right)$ for some $\alpha>0, \omega=0$ and arbitrary $(0, v) \in E_{2}$.

Lemma 4.5. Assume that $\left(V_{1}\right)_{i}-\left(V_{3}\right)_{i},(V)$ and $\left(F_{1}^{\prime}\right)-\left(F_{2}^{\prime}\right)$ hold, then I satisfies $\left(I_{3}\right)$.

Although $\left(I_{3}\right)(i)$ holds for $I$ by the same argument found in Lemma 4.3 , for the sake of completeness, this proof is repeated here.

Proof. From (4.6) and 4.5), for all $\left(u_{1}, 0\right) \in S$, it yields

$$
\begin{aligned}
I\left(u_{1}, 0\right) & =\frac{1}{2}\left\|\left(u_{1}, 0\right)\right\|_{E}^{2}-\int_{\mathbb{R}^{N}} F\left(x, u_{1}(x), 0\right) d x \\
& \geq \frac{1}{2} \rho^{2}-\left(\varepsilon\left\|\left(u_{1}, 0\right)\right\|_{E}^{2}+C_{\varepsilon}\left\|\left(u_{1}, 0\right)\right\|_{E}^{p}\right) \\
& =\rho^{2}\left[\left(\frac{1}{2}-\varepsilon\right)-C_{\varepsilon} \rho^{p-2}\right] \\
& \geq \alpha>0
\end{aligned}
$$

where $\varepsilon, \rho$ are chosen small enough. Therefore, $\left(I_{3}\right)(i)$ holds for $I$.

For showing $\left(I_{3}\right)(i i)$ with $\omega=0$, since $I(0, v) \leq 0$, for all $(0, v) \in E_{2}$, it is enough to prove that $I(r e, v) \leq 0$ for $r>0,(0, v) \in E_{2}$ and $\|(r e, v)\|_{E} \geq r_{1}$, for 
some $r_{1}>0$ sufficiently large. Seeking a contradiction, assume that some sequence $\left(r_{n} e, v_{n}\right) \subset \mathbb{R}^{+} e \oplus E_{2}$ satisfies $\left\|\left(r_{n} e, v_{n}\right)\right\|_{E} \rightarrow+\infty$ and $I\left(r_{n} e, v_{n}\right)>0$ for all $n \in \mathbb{N}$. Set

$$
\left(s_{n} e, w_{n}\right):=\frac{\left(r_{n} e, v_{n}\right)}{\left\|\left(r_{n} e, v_{n}\right)\right\|_{E}}
$$

where $s_{n} \in \mathbb{R}^{+},\left(0, w_{n}\right) \in E_{2}$ and $\left\|\left(s_{n} e, w_{n}\right)\right\|_{E}=1$. Provided that $\left(s_{n} e, w_{n}\right)$ is bounded, up to subsequences, it follows that $\left(s_{n} e, w_{n}\right) \rightarrow(s e, w)$ in $E$, hence $\left(s_{n} e, w_{n}\right) \rightarrow(s e, w)$ in $L_{h(x)}^{2}\left(\mathbb{R}^{N}, \mathbb{R}^{2}\right)$, due to the compact embedding $E \hookrightarrow L_{h(x)}^{2}\left(\mathbb{R}^{N}, \mathbb{R}^{2}\right)$. Then, up to subsequences, $\left(s_{n} e(x), w_{n}(x)\right) \rightarrow(s e(x), w(x))$ almost everywhere in $\mathbb{R}^{N}, s_{n} \rightarrow s$ in $\mathbb{R}^{+}$ and $\left(0, w_{n}\right) \rightarrow(0, w)$ in $E_{2}$. Since $1=\left\|\left(s_{n} e, w_{n}\right)\right\|_{E}^{2}=s_{n}^{2}+\left\|\left(0, w_{n}\right)\right\|_{E}^{2}$, it follows that $0 \leq s_{n}^{2} \leq 1$, and it yields

$$
\begin{aligned}
0<\frac{I\left(r_{n} e, v_{n}\right)}{\left\|\left(r_{n} e, v_{n}\right)\right\|_{E}^{2}} & =\frac{1}{2}\left(s_{n}^{2}\|(e, 0)\|_{E}^{2}-\left\|\left(0, w_{n}\right)\right\|_{E}^{2}\right)-\int_{\mathbb{R}^{N}} \frac{F\left(x, r_{n} e(x), v_{n}(x)\right)}{\left\|\left(r_{n} e, v_{n}\right)\right\|_{E}^{2}} d x \\
& =s_{n}^{2}-\frac{1}{2}-\int_{\mathbb{R}^{N}} \frac{F\left(x, r_{n} e(x), v_{n}(x)\right)}{\left\|\left(r_{n} e, v_{n}\right)\right\|_{E}^{2}} d x \\
& =s_{n}^{2}-\frac{1}{2}-\int_{\mathbb{R}^{N}} \frac{F\left(x, r_{n} e(x), v_{n}(x)\right)}{\left|\left(r_{n} e(x), v_{n}(x)\right)\right|^{2}}\left|\left(s_{n} e(x), w_{n}(x)\right)\right|^{2} d x,
\end{aligned}
$$

hence $\frac{1}{2} \leq s^{2} \leq 1$. Provided that $\left\|\left(r_{n} e, v_{n}\right)\right\|_{E} \rightarrow+\infty$ and

$$
\left(s_{n} e(x), w_{n}(x)\right) \rightarrow(s e(x), w(x)) \neq 0
$$

almost everywhere in $\operatorname{supp}(e)$, it follows that

$$
\left(r_{n} e(x), v_{n}(x)\right)=\left(s_{n} e(x), w_{n}(x)\right)\left\|\left(r_{n} e, v_{n}\right)\right\|_{E} \rightarrow+\infty,
$$

almost everywhere in $\operatorname{supp}(e)$, as $n \rightarrow+\infty$, hence in view of $\left(F_{2}^{\prime}\right)$ one has

$$
\frac{F\left(x, r_{n} e(x), v_{n}(x)\right)}{\left|\left(r_{n} e(x), v_{n}(x)\right)\right|^{2}}\left|\left(s_{n} e(x), w_{n}(x)\right)\right|^{2} \rightarrow+\infty,
$$

almost everywhere in $\operatorname{supp}(s e, w)$ as $n \rightarrow+\infty$. Note that, $\operatorname{supp}(s e, w) \neq \emptyset$, because $\operatorname{supp}(e) \neq \emptyset$. Thus, by Fatou's Lemma,

$$
\int_{\mathbb{R}^{N}} \frac{F\left(x, r_{n} e(x), v_{n}(x)\right)}{\left|\left(r_{n} e(x), v_{n}(x)\right)\right|^{2}}\left|\left(s_{n} e(x), w_{n}(x)\right)\right|^{2} d x \rightarrow+\infty,
$$

as $n \rightarrow+\infty$. In virtue of 4.26 one has 


$$
\frac{1}{2} \geq s_{n}^{2}-\frac{1}{2}>\int_{\mathbb{R}^{N}} \frac{F\left(x, r_{n} e(x), v_{n}(x)\right)}{\left|\left(r_{n} e(x), v_{n}(x)\right)\right|^{2}}\left|\left(s_{n} e(x), w_{n}(x)\right)\right|^{2} d x .
$$

Due to (4.27), passing to the limit as $n \rightarrow+\infty$ it yields a contradiction.

Remark 4.6. Note that the assumption $a_{0}>\lambda_{1}^{1}$ in $(V)$, is irrelevant in the super quadratic case, since inequality (4.14) is not used to establish the linking geometry as was done in Lemma 4.3. Therefore, for the super quadratic case, it is just necessary to require $a_{0}>0$ in $(V)$. Thereby, if $F \leq 0$ were considered instead of $F \geq 0$, to treat the problem would be necessary only to exchange one equation for the another, requiring hypothesis $\left(F_{2}^{\prime}\right)$ for $-F \geq 0$ instead of $F$.

\subsubsection{Boundedness of Cerami Sequences}

Last hypothesis in Theorem 1.4 is ensured in this subsection, and subsequently Theorem 4.2 is going to be proved. Two lemmas are proved, the first one assuming $\left(F_{3}^{\prime}\right)$, and the second one considering hypothesis $\left(F_{3}^{\prime \prime}\right)$ instead.

Lemma 4.6. Under the hypotheses $\left(V_{1}\right)_{i}-\left(V_{3}\right)_{i},(V)$ and $\left(F_{1}^{\prime}\right)-\left(F_{3}^{\prime}\right)$, I satisfies $\left(I_{4}\right)$.

Proof. Let $b>0$ be a constant such that $c-b>0$ and take $\left(u_{n}, v_{n}\right) \subset I^{-1}([c-b, c+b])$ such that $\left(1+\left\|\left(u_{n}, v_{n}\right)\right\|_{E}\right)\left\|I^{\prime}\left(u_{n}, v_{n}\right)\right\|_{E^{\prime}} \rightarrow 0$, it is necessary to show that $\left(u_{n}, v_{n}\right)$ is bounded. Since $\left(u_{n}, v_{n}\right)$ is a Cerami sequence, it satisfies

$$
o_{n}(1)=I^{\prime}\left(u_{n}, v_{n}\right)\left(u_{n}, 0\right)=\left\|\left(u_{n}, 0\right)\right\|_{E}^{2}-\int_{\mathbb{R}^{N}} F_{u}\left(x, u_{n}(x), v_{n}(x)\right) u_{n}(x) d x
$$

and

$$
o_{n}(1)=I^{\prime}\left(u_{n}, v_{n}\right)\left(0, v_{n}\right)=-\left\|\left(0, v_{n}\right)\right\|_{E}^{2}-\int_{\mathbb{R}^{N}} F_{v}\left(x, u_{n}(x), v_{n}(x)\right) v_{n}(x) d x
$$

Subtracting 4.29) from 4.28), and using $\left(F_{1}^{\prime}\right)$, it yields

$$
\begin{aligned}
\left\|\left(u_{n}, v_{n}\right)\right\|_{E}^{2} & =o_{n}(1)+\int_{\mathbb{R}^{N}}\left(F_{u}\left(x, u_{n}(x), v_{n}(x)\right) u_{n}(x)-F_{v}\left(x, u_{n}(x), v_{n}(x)\right) v_{n}(x)\right) d x \\
& \leq m_{0}+\int_{\mathbb{R}^{N}}\left|\left(F_{u}\left(x, u_{n}(x), v_{n}(x)\right), F_{v}\left(x, u_{n}(x), v_{n}(x)\right)\right)\right| \mid\left(u_{n}(x), v_{n}(x) \mid d x\right. \\
& \leq m_{0}+c_{0} \int_{\mathbb{R}^{N}} h(x)\left(\varepsilon\left|\left(u_{n}(x), v_{n}(x)\right)\right|^{2}+C_{\varepsilon}\left|\left(u_{n}(x), v_{n}(x)\right)\right|^{p}\right) d x \\
& \leq m_{0}+c_{0} C_{2}^{2} \varepsilon\left\|\left(u_{n}, v_{n}\right)\right\|_{E}^{2}+c_{0} C_{\varepsilon}\left\|\left(u_{n}, v_{n}\right)\right\|_{L_{h(x)}^{p}\left(\mathbb{R}^{N}, \mathbb{R}^{2}\right)}^{p}
\end{aligned}
$$


for arbitrary $\varepsilon>0$, some constants $m_{0}, C_{\varepsilon}>0$ and for $C_{2}>0$ a constant given by the embedding $E \hookrightarrow L_{h(x)}^{2}\left(\mathbb{R}^{N}, \mathbb{R}^{2}\right)$. Hence, in view of 4.30 , one has

$$
\left(1-c_{0} C_{2}^{2} \varepsilon\right)\left\|\left(u_{n}, v_{n}\right)\right\|_{E}^{2} \leq m_{0}+c_{0} C_{\varepsilon}\left\|\left(u_{n}, v_{n}\right)\right\|_{L_{h(x)}^{p}\left(\mathbb{R}^{N}, \mathbb{R}^{2}\right)} \cdot
$$

On the other hand, observe that from $\left(F_{2}^{\prime}\right)$ and since $\left(u_{n}, v_{n}\right)$ is a Cerami sequence, for some constant $M>0$, one has

$$
\begin{aligned}
M & \geq I\left(u_{n}, v_{n}\right)-\frac{1}{2} I^{\prime}\left(u_{n}, v_{n}\right)\left(u_{n}, v_{n}\right) \\
& =\frac{1}{2} \int_{\mathbb{R}^{N}}\left(F_{u}\left(x, u_{n}(x), v_{n}(x)\right) u_{n}(x)+F_{v}\left(x, u_{n}(x), v_{n}(x)\right) v_{n}(x)\right) d x \\
& -\int_{\mathbb{R}^{N}} F\left(x, u_{n}(x), v_{n}(x)\right) d x \\
& =\frac{1}{2} \int_{\mathbb{R}^{N}} Q\left(x, u_{n}(x), v_{n}(x)\right) d x \\
& \geq \frac{c_{1}}{2} \int_{\mathbb{R}^{N}} h(x)\left|\left(u_{n}(x), v_{n}(x)\right)\right|^{\theta} d x \\
& =\frac{c_{1}}{2}\left\|\left(u_{n}, v_{n}\right)\right\|_{L_{h(x)}^{\theta}\left(\mathbb{R}^{N}, \mathbb{R}^{2}\right)}^{\theta}
\end{aligned}
$$

thus, $\left(u_{n}, v_{n}\right)$ is a bounded sequence in $L_{h(x)}^{\theta}\left(\mathbb{R}^{N}, \mathbb{R}^{2}\right)$. Observe that, if $p=\theta$ from 4.31 one has $\left(u_{n}, v_{n}\right)$ bounded in $E$. If not, first consider the case $2<p<\theta$, then there exists $t \in(0,1)$ such that $p=t 2+(1-t) \theta$, hence applying Hölder Inequality and in view of 4.32 it implies that

$$
\begin{aligned}
\left\|\left(u_{n}, v_{n}\right)\right\|_{L_{h(x)}^{p}}^{p}\left(\mathbb{R}^{N}, \mathbb{R}^{2}\right) & \leq\left\|\left(u_{n}, v_{n}\right)\right\|_{L_{h(x)}^{2}\left(\mathbb{R}^{N}, \mathbb{R}^{2}\right)}^{2 t}\left\|\left(u_{n}, v_{n}\right)\right\|_{L_{h(x)}^{\theta}(1-t) \theta}^{\left(\mathbb{R}^{N}, \mathbb{R}^{2}\right)} \\
& \leq\left(\frac{2 M}{c_{1}}\right)^{1-t} C_{2}^{2 t}\left\|\left(u_{n}, v_{n}\right)\right\|_{E}^{2 t} .
\end{aligned}
$$

Substituting 4.33 in 4.31, it yields

$$
\left(1-c_{0} C_{2}^{2} \varepsilon\right)\left\|\left(u_{n}, v_{n}\right)\right\|_{E}^{2} \leq m_{0}+c_{0} C_{\varepsilon}\left(\frac{2 M}{c_{1}}\right)^{1-t} C_{2}^{2 t}\left\|\left(u_{n}, v_{n}\right)\right\|_{E}^{2 t},
$$

and since $2 t<2$, choosing $\varepsilon>0$ small enough, it ensures that $\left(u_{n}, v_{n}\right)$ is bounded in $E$. Now, it remains to consider the case $\theta<p<2^{\#}$, then there exists $t \in(0,1)$ such that $p=t 2^{\#}+(1-t) \theta$. Again from Hölder Inequality and using the boundedness in 4.32 it follows that 


$$
\begin{aligned}
\left\|\left(u_{n}, v_{n}\right)\right\|_{L_{h(x)}^{p}\left(\mathbb{R}^{N}, \mathbb{R}^{2}\right)}^{p} & \leq\left\|\left(u_{n}, v_{n}\right)\right\|_{L_{h(x)}^{2 \#}\left(\mathbb{R}^{N}, \mathbb{R}^{2}\right)}^{\#}\left\|\left(u_{n}, v_{n}\right)\right\|_{L_{h(x)}^{\theta}(1-t) \theta}^{\left(\mathbb{R}^{N}, \mathbb{R}^{2}\right)} \\
& \leq\left(\frac{2 M}{c_{1}}\right)^{1-t} C_{2^{\#}}^{t 2^{\#}}\left\|\left(u_{n}, v_{n}\right)\right\|_{E}^{t 2^{\#}}
\end{aligned}
$$

where $C_{2 \#}>0$ is the constant given by the embedding $E \hookrightarrow L_{h(x)}^{2^{\#}}\left(\mathbb{R}^{N}, \mathbb{R}^{2}\right)$. Substituting 4.35 in 4.31), it yields

$$
\left(1-c_{0} C_{2}^{2} \varepsilon\right)\left\|\left(u_{n}, v_{n}\right)\right\|_{E}^{2} \leq m_{0}+c_{0} C_{\varepsilon}\left(\frac{2 M}{c_{1}}\right)^{1-t} C_{2}^{t 2^{\#}}\left\|\left(u_{n}, v_{n}\right)\right\|_{E}^{t 2 \#},
$$

and $t 2^{\#}<2$, provided that $\theta>\frac{N}{2}(p-2)$, hence choosing $\varepsilon>0$ small enough, it ensures that also in this case $\left(u_{n}, v_{n}\right)$ is bounded in $E$. Therefore, the result holds.

Lemma 4.7. Supposing that $\left(V_{1}\right)_{i}-\left(V_{3}\right)_{i},(V),\left(F_{1}^{\prime}\right)-\left(F_{2}^{\prime}\right)$ and $\left(F_{3}^{\prime \prime}\right)$ hold true for $I$, it satisfies $\left(I_{4}\right)$.

Proof. Let $b>0$ be a constant such that $c-b>0$ and take $\left(u_{n}, v_{n}\right) \subset I^{-1}([c-b, c+b])$ such that $\left(1+\left\|\left(u_{n}, v_{n}\right)\right\|_{E}\right)\left\|I^{\prime}\left(u_{n}, v_{n}\right)\right\|_{E^{\prime}} \rightarrow 0$, it is necessary to show that $\left(u_{n}, v_{n}\right)$ is bounded. Seeking a contradiction, suppose that $\left\|\left(u_{n}, v_{n}\right)\right\|_{E} \rightarrow+\infty$, up to subsequences and define $\left(\tilde{u}_{n}, \tilde{v}_{n}\right):=\frac{\left(u_{n}, v_{n}\right)}{\left\|\left(u_{n}, v_{n}\right)\right\|_{E}}$, the normalized sequence. Since it is bounded, $\left(\tilde{u}_{n}, \tilde{v}_{n}\right) \rightarrow(\tilde{u}, \tilde{v})$ in $E$, up to subsequences, and due to the compact embeddings $E \hookrightarrow L_{h(x)}^{s}\left(\mathbb{R}^{N}, \mathbb{R}^{2}\right)$ for $2 \leq s<+\infty$ if $N=2$ and $2 \leq s<2^{\#}$ if $N>2$, it implies that $\left(\tilde{u}_{n}, \tilde{v}_{n}\right) \rightarrow(\tilde{u}, \tilde{v})$ in $L_{h(x)}^{s}\left(\mathbb{R}^{N}, \mathbb{R}^{2}\right)$.

Suppose that $(\tilde{u}(x), \tilde{v}(x))=(0,0)$ almost everywhere in $\mathbb{R}^{N}$ and for $\varepsilon \in(0,1)$ define $U_{n}^{\varepsilon}=\left(t_{n}^{\varepsilon} u_{n}, \varepsilon t_{n}^{\varepsilon} v_{n}\right) \in E$ such that

$$
I\left(U_{n}^{\varepsilon}\right)=\max _{t \in[0,1]} I\left(t u_{n}, \varepsilon t v_{n}\right)
$$

Provided that $(\tilde{u}, \tilde{v}) \rightarrow 0$ in $L_{h(x)}^{s}\left(\mathbb{R}^{N}, \mathbb{R}^{2}\right)$, from $\left(F_{1}^{\prime}\right)$ for each $s>0$ fixed, there exists $C_{s}>0$, which does not depend on $\varepsilon$, such that

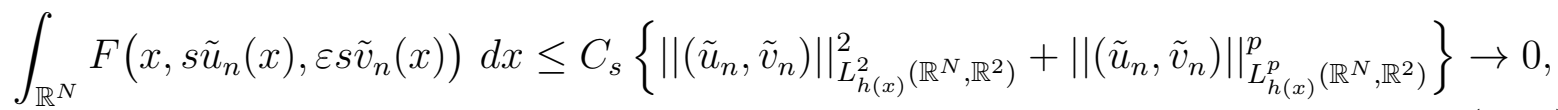


as $n \rightarrow+\infty$. Moreover, since $\left\|\left(u_{n}, v_{n}\right)\right\|_{E} \rightarrow+\infty$, for $n$ large enough one has

$$
\begin{aligned}
I\left(U_{n}^{\varepsilon}\right) & \geq I\left(\frac{s u_{n}}{\left\|\left(u_{n}, v_{n}\right)\right\|_{E}}, \frac{\varepsilon s v_{n}}{\left\|\left(u_{n}, v_{n}\right)\right\|_{E}}\right) \\
& =\frac{s^{2}}{2}\left(\left\|\left(\tilde{u}_{n}, 0\right)\right\|_{E}^{2}-\varepsilon^{2}\left\|\left(0, \tilde{v}_{n}\right)\right\|_{E}^{2}\right)-\int_{\mathbb{R}^{N}} F\left(x, s \tilde{u}_{n}(x), \varepsilon s \tilde{v}_{n}(x)\right) d x
\end{aligned}
$$

Since $I\left(u_{n}, v_{n}\right) \geq c-b>0$, it follows that $\left\|\left(\tilde{u}_{n}, 0\right)\right\|_{E}^{2}>\left\|\left(0, \tilde{v}_{n}\right)\right\|_{E}^{2}$. Combining this inequality with $1=\left\|\left(\tilde{u}_{n}, 0\right)\right\|_{E}^{2}+\left\|\left(0, \tilde{v}_{n}\right)\right\|_{E}^{2}$, it implies that $\left\|\left(\tilde{u}_{n}, 0\right)\right\|_{E}^{2}>\frac{1}{2}$. Hence, from (4.38) and 4.39) it implies that

$$
\begin{aligned}
\liminf _{n \rightarrow+\infty} I\left(U_{n}^{\varepsilon}\right) & \geq \frac{s^{2}}{2} \liminf _{n \rightarrow+\infty}\left(\left\|\left(\tilde{u}_{n}, 0\right)\right\|_{E}^{2}-\varepsilon^{2}\left\|\left(\tilde{u}_{n}, 0\right)\right\|_{E}^{2}\right) \\
& =\frac{s^{2}}{2}\left(1-\varepsilon^{2}\right) \liminf _{n \rightarrow+\infty}\left\|\left(\tilde{u}_{n}, 0\right)\right\|_{E}^{2} \\
& \geq \frac{s^{2}}{4}\left(1-\varepsilon^{2}\right),
\end{aligned}
$$

for all $s>0$ and $\varepsilon \in(0,1)$. Thus,

$$
\liminf _{n \rightarrow+\infty} I\left(U_{n}^{\varepsilon}\right)=+\infty \quad \text { for all } \quad \varepsilon \in(0,1)
$$

On the other hand, if there exists a subsequence $\left(n_{k}\right) \subset \mathbb{N}$, such that for some $\varepsilon_{n_{k}} \in(0,1)$ one has $t_{n_{k}}^{\varepsilon_{n_{k}}} \in(0,1)$, in view of 4.37) it implies that $I^{\prime}\left(U_{n_{k}}^{\varepsilon_{n_{k}}}\right) U_{n_{k}}^{\varepsilon_{n_{k}}}=0$. In addition, since $t_{n_{k}}^{\varepsilon_{n_{k}}} \in(0,1)$, in virtue of $\left(F_{3}^{\prime \prime}\right)$ it follows that

$$
\begin{aligned}
I\left(U_{n_{k}}^{\varepsilon_{n_{k}}}\right) & =I\left(U_{n_{k}}^{\varepsilon_{n_{k}}}\right)-\frac{1}{2} I^{\prime}\left(U_{n_{k}}^{\varepsilon_{n_{k}}}\right) U_{n_{k}}^{\varepsilon_{n_{k}}} \\
& =\frac{1}{2} \int_{\mathbb{R}^{N}} Q\left(x, t_{n_{k}}^{\varepsilon_{n_{k}}} u_{n_{k}}(x), \varepsilon_{n_{k}} t_{n_{k}}^{\varepsilon_{n_{k}}} v_{n_{k}}(x)\right) d x \\
& \leq \frac{D}{2} \int_{\mathbb{R}^{N}} Q\left(x, u_{n_{k}}(x), v_{n_{k}}(x)\right) d x \\
& =\frac{D}{2}\left\{I\left(u_{n_{k}}, v_{n_{k}}\right)-\frac{1}{2} I^{\prime}\left(u_{n_{k}}, v_{n_{k}}\right)\left(u_{n_{k}}, v_{n_{k}}\right)\right\} \\
& \leq M
\end{aligned}
$$

for some $M>0$, provided that $\left(u_{n_{k}}, v_{n_{k}}\right)$ is also a Cerami sequence. However, from 4.41) one arrives at

$$
\limsup _{k \rightarrow+\infty} I\left(U_{n_{k}}^{\varepsilon_{n_{k}}}\right) \leq M
$$

contradicting 4.40. Thus, such a subsequence $\left(n_{k}\right) \subset \mathbb{N}$ cannot exist and hence, there exists $n_{0} \in \mathbb{N}$ such that for all $n \geq n_{0}$ and for all $\varepsilon \in(0,1)$ one has $t_{n}^{\varepsilon}=1$. Furthermore, 
by the continuity of $I$ it yields

$$
I\left(U_{n}^{\varepsilon}\right)=I\left(u_{n}, \varepsilon v_{n}\right) \rightarrow I\left(u_{n}, v_{n}\right) \quad \text { as } \quad \varepsilon \rightarrow 1
$$

for each $n \geq n_{0}$. Then, for each $n \geq n_{0}$ there exists $\varepsilon_{n} \in(0,1)$ such that

$$
I\left(U_{n}^{\varepsilon}\right)<I\left(u_{n}, v_{n}\right)+\frac{1}{n}
$$

for all $\varepsilon \in\left[\varepsilon_{n}, 1\right]$. Hence, if $\tilde{\varepsilon}_{n} \in\left[\varepsilon_{n}, 1\right]$ is such that $I\left(U_{n}^{\tilde{\varepsilon}_{n}}\right) \geq I\left(U_{n}^{\varepsilon}\right)$ for all $\varepsilon \in\left[\varepsilon_{n}, 1\right]$, in view of 4.42 it follows that

$$
\limsup _{n \rightarrow+\infty} I\left(U_{n}^{\tilde{\varepsilon}_{n}}\right) \leq \limsup _{n \rightarrow+\infty} I\left(u_{n}, v_{n}\right)=c
$$

However, from (4.40) for each $\varepsilon \in\left[\varepsilon_{n}, 1\right)$ there exists $n_{\varepsilon} \in \mathbb{N}$ such that $n \geq n_{\varepsilon}$ implies $I\left(U_{n}^{\varepsilon}\right) \geq \frac{1}{1-\varepsilon} \geq \frac{1}{1-\varepsilon_{n}}$, then, it yields

$$
\liminf _{n \rightarrow+\infty} I\left(U_{n}^{\tilde{\varepsilon}_{n}}\right) \geq \liminf _{n \rightarrow+\infty} I\left(U_{n}^{\varepsilon}\right) \geq \lim _{n \rightarrow+\infty} \frac{1}{1-\varepsilon_{n}}=+\infty
$$

since $\varepsilon_{n} \rightarrow 1$, in virtue of 4.42). Thus, 4.44 contradicts 4.43).

Therefore, $(\tilde{u}, \tilde{v})$ cannot be the null function, namely there exists $\Omega \subset \mathbb{R}^{N}$ with $|\Omega|>0$, such that $(\tilde{u}(x), \tilde{v}(x)) \neq(0,0)$ for all $x \in \Omega$. Furthermore, since

$$
\begin{aligned}
\int_{\mathbb{R}^{N}} \frac{F\left(x, u_{n}(x), v_{n}(x)\right)}{\left\|\left(u_{n}, v_{n}\right)\right\|_{E}^{2}} d x & =\frac{1}{2}\left(\left\|\left(\tilde{u}_{n}, 0\right)\right\|_{E}^{2}-\left\|\left(0, \tilde{v}_{n}\right)\right\|_{E}^{2}\right)-\frac{I\left(u_{n}, v_{n}\right)}{\left\|\left(u_{n}, v_{n}\right)\right\|_{E}^{2}} \\
& \leq \frac{1}{2}\left\|\left(\tilde{u}_{n}, 0\right)\right\|_{E}^{2} \leq \frac{1}{2},
\end{aligned}
$$

one has

$$
\frac{1}{2} \geq \int_{\Omega} \frac{F\left(x, u_{n}(x), v_{n}(x)\right)}{\left|\left(u_{n}(x), v_{n}(x)\right)\right|^{2}}\left|\left(\tilde{u}_{n}, \tilde{v}_{n}\right)\right|^{2} d x .
$$

Nevertheless, in view of $\left(F_{2}^{\prime}\right)$, for all $x \in \Omega$ it implies that

$$
\frac{F\left(x, u_{n}(x), v_{n}(x)\right)}{\left|\left(u_{n}(x), v_{n}(x)\right)\right|^{2}}\left|\left(\tilde{u}_{n}, \tilde{v}_{n}\right)\right|^{2} \rightarrow+\infty
$$

as $n \rightarrow+\infty$, provided that $\left|\left(u_{n}(x), v_{n}(x)\right)\right|=\left|\left(\tilde{u}_{n}(x), \tilde{v}_{n}(x)\right)\right| \|\left(u_{n}, v_{n}\right)||_{E} \rightarrow+\infty$ for all $x \in \Omega$, as $n \rightarrow+\infty$. Passing 4.45 to the limit and applying Fatou's Lemma, it yields

$$
\frac{1}{2} \geq \liminf _{n \rightarrow+\infty} \int_{\Omega} \frac{F\left(x, u_{n}(x), v_{n}(x)\right)}{\left|\left(u_{n}(x), v_{n}(x)\right)\right|^{2}}\left|\left(\tilde{u}_{n}, \tilde{v}_{n}\right)\right|^{2} d x=+\infty,
$$


which is a contradiction. Therefore, $\left(u_{n}, v_{n}\right)$ is bounded, and the result holds.

At long last, the main result is proved.

Proof of Theorem 4.2. Provided that $I$ satisfies all assumptions $\left(I_{1}\right)-\left(I_{4}\right)$ in Theorem 1.4, it ensures a critical point $(u, v) \in E$ of $I$, with $I(u, v)=c \geq \alpha>0$. It implies that $I^{\prime}(u, v)(\varphi, \psi)=0$, for all $(\varphi, \psi) \in E$ and $I(u, v)>0=I(0,0)$, thus $(u, v) \neq 0$. Since $I \in C^{1}(E, \mathbb{R})$, it yields that $(u, v)$ is a nontrivial solution to system $(E S)$ in 4.1 .

It is worth to mention that depending on the nonlinearity, the solutions $(u, v)$ found in Theorem 4.1 or in Theorem 4.2 could be either semi-trivial $(u, 0)$ or vectorial $(u, v)$, but never semi-trivial $(0, v)$, since such solutions have positive energy $c>0$. 


\section{Auxiliary Results}

In this chapter, as a matter of completeness are stated the most important auxiliary results used along with this work.

\section{Theorem 1.1'. [9], page 394}

Let $A$ be a self-adjoint operator in Hilbert space $H$. Then

1) there is a spectral family $E_{\lambda},-\infty<\lambda<+\infty$, that is, a family of self-adjoint projection operators $E_{\lambda}$ in $H$ depending on a real parameter $\lambda$ satisfying

a) $E_{\lambda} E_{\mu}=E_{\mu} E_{\lambda}=E_{\lambda}$ for $\mu<\lambda$;

b) $E_{\lambda+0}=E_{\lambda}$ in the strong operator topology, that is

$$
\lim _{\varepsilon \rightarrow 0^{+}} E_{\lambda+\varepsilon} f=E_{\lambda} f
$$

in the norm of $H$ for any $f \in H$;

c) in the strong operator topology we have

$$
\lim _{\lambda \rightarrow-\infty} E_{\lambda}=0, \quad \lim _{\lambda \rightarrow+\infty} E_{\lambda}=I
$$

d) if $\Delta=\left(\lambda_{1}, \lambda_{2}\right]$ is a half-open interval on the real axis, $-\infty<\lambda_{1}<\lambda_{2}<+\infty$ and $E(\Delta)=E_{\lambda_{2}}-E_{\lambda_{1}}$, then $E(\Delta) H \subset D(A)$ and for $f \in E(\Delta) H$ the inequalities

$$
\lambda_{1}(f, f) \leq(A f, f) \leq \lambda_{2}(f, f)
$$

and the estimate

$$
\|(A-\lambda I) f\| \leq\left|\lambda_{2}-\lambda_{1}\right|\|f\|
$$

hold for $\lambda \in \Delta$. This means that vectors in $E(\Delta) H$ for small $\Delta$ are almost eigenvectors 
of $A$ with the eigenvalue $\lambda \in \Delta$;

e) $A$ is recovered from the family $\left\{E_{\lambda}\right\}$ by the formula

$$
A=\int_{-\infty}^{+\infty} \lambda d E_{\lambda}
$$

which means that $f \in H$ belongs to $D(A)$ if and only if the integral

$$
\int_{-\infty}^{+\infty} \lambda^{2} d\left(E_{\lambda} f, f\right)=\int_{-\infty}^{+\infty} \lambda^{2} d\left\|E_{\lambda} f\right\|^{2}<+\infty
$$

converges. Moreover, the left-hand side of this inequality equals $\|A f\|^{2}$ and

$$
A(f)=\int_{-\infty}^{+\infty} \lambda d\left(E_{\lambda} f\right)
$$

where the integral is to be understood as

$$
\lim _{\alpha \rightarrow-\infty, \beta \rightarrow+\infty} \int_{\alpha}^{\beta} \lambda d\left(E_{\lambda} f\right)
$$

with respect to the norm in $H$, while integrals along a finite interval are just limits of their integral sums with respect to the norm in $H$ and they even are uniform in all $f$ for $\|f\|<1$

2) The spectral family with properties $a)-e$ ) is unique.

\section{Theorem 30. [23], page 150}

If a measurable locally bounded function $V(x)$ is such that

$$
\liminf _{|x| \rightarrow+\infty} V(x) \geq a
$$

then the operator $L=-\Delta+V(x)$ is semi-bounded from below and has a discrete spectrum on $(-\infty, a)$, so that for any $\varepsilon>0$ the spectrum of $L$ on $(-\infty, a-\varepsilon)$ consists of a finite number of eigenvalues of finite multiplicities.

\section{Corollary 11.3.6 (Weyl) [37], page 290}

Let $T$ be self-adjoint and $B$ hermitian. If $B$ is $T$-compact, then

$$
\sigma_{e s s}(T+B)=\sigma_{e s s}(T)
$$




\section{Proposition 6.6. [43] page 36}

For each $s \in[1,+\infty), E=W^{\frac{1}{2}, 2}\left(S^{1}, \mathbb{R}^{2 N}\right)$ is compactly embedded in $L^{s}\left(S^{1}, \mathbb{R}^{2 N}\right)$. In particular there is an $\alpha_{s}>0$ such that

$$
\|z\|_{L^{s}} \leq \alpha_{s}\|z\|
$$

for all $z \in E$.

\section{Corollary 2 [44], page 113}

Let $A$ be a self-adjoint operator and let $C$ be a relatively compact perturbation of $A$. Then:

(a) $B=A+C$ defined with $D(B)=D(A)$ is a closed operator;

(b) If $C$ is symmetric, $B$ is self-adjoint;

(c) $\sigma_{\text {ess }}(A)=\sigma_{\text {ess }}(B)$.

\section{Theorem 3.15 [51], page 44}

Let $V \in L^{\infty}, S:=\Delta+V(x)$ and

$$
\operatorname{limess}_{R \rightarrow+\infty} \inf _{|x| \geq R} V(x)=l \text {. }
$$

(i) Then $\sigma_{e}(S) \subset[l,+\infty)$;

(ii) If

$$
\operatorname{limess}_{R \rightarrow+\infty} \inf _{|x| \geq R}|V(x)-l|=0, \quad \text { then } \sigma_{e}(S)=[l,+\infty) .
$$

\section{Lemma 1.21 (Lions' Lemma) [57], page 16}

Let $r>0$ and $2 \leq q<2^{*}$. If $\left(u_{n}\right)$ is bounded in $H^{1}\left(\mathbb{R}^{N}\right)$ and if

$$
\sup _{y \in \mathbb{R}^{N}} \int_{B(y, r)}\left|u_{n}\right|^{q} \rightarrow 0, \quad n \rightarrow+\infty
$$

then $u_{n} \rightarrow 0$ in $L^{p}\left(\mathbb{R}^{N}\right)$ for $2<p<2^{*}$. 


\section{Bibliography}

[1] Ambrosetti, A. and Rabinowitz, P. H.: Dual Variational Methods in Critical Point Theory and Applications. J. Functional Analysis, 14, 349-381, 1973. 11

[2] Azzollini, A. e Pomponio, A.: On the Schrödinger Equation in $\mathbb{R}^{N}$ under the Effect of a General Nonlinear Term. Indiana University Mathematics Journal 58 No. 3, 1361-1378, 2009. 7

[3] Bartolo, P., Benci, V. and Fortunato, D.: Abstract Critical Point Theorems and Applications to some Nonlinear Problems with "Strong" Resonance at Infinity. Nonlinear Analysis Theory, Methods \& Applications 7, 981-1012, 1983. 2, 8

[4] Bartsch, T. and Clapp, M.: Critical Point Theory for Indefinite Functionals with Symmetries. J. Func. Anal. 138, 107-136, 1996. 11

[5] Bartsch, T. and Ding, Y.H.: Deformation Theorems on Non-metrizable Vector Spaces and Applications to Critical Point Theory. Math. Nachr. 279, 1267-1288, 2006. 3, 6

[6] Bartsch, T. and Willem, M.: Infinitely Many Radial Solutions of a Semilinear Elliptic Problem on $\mathbb{R}^{N}$. Arch. Rational Mech. Anal. 124, 261-276, 1993. 7

[7] Benci, V. and Rabinowitz, P. H.: Critical Point Theorems for Indefinite Functionals. Inventiones Math. 52, 241-273, 1979. 1, 2, 3, 4, 12, 13, 15, 22, 27, 28, 30

[8] Berestycki, H. and Lions, P. L.: Nonlinear Scalar Field Equations I. Arch. Rat. Mech. Anal. 82, 313-346, 1983. 7, 67, 69, 71, 72, 76

[9] Berezin, F. A. and Shubin, M. A.: The Schrödinger Equation, Kluwer Academic Publishers, 1991. 28, 37, 46, 47, 59, 60, 101 
[10] Brezis, H. and Lieb, E. H.: A Relation Between Pointwise Convergence of Functions and Convergence of Functionals. Proc. Amer. Math. Soc. 88 No. 3, 486-490, 1983. 53

[11] Cerami, G.: Un Criterio di Esistenza per i Punti Critici su Varietà Illimitate. Rc. Ist. Lomb. Sci. Lett. 112, 332-336, 1978. 1

[12] Chang, S. M., Lin, C. S. Lin, T. C. and Lin, W. W.: Segregated Nodal Domains of Two-dimensional Multi-species Bose-Einstein Condensates. Physica D 196, 341-361, 2004. 10

[13] Chen, S. and Zhang, D.: Existence of nontrivial solutions for asymptotically linear periodic Schrödinger equations. Complex Variables and Elliptic Equations: An International Journal 60, 252-267, 2015. 3

[14] Costa, D. G.: On a Class of Elliptic Systems in $\mathbb{R}^{N}$. Electronic Journal of Differential Equations 7, 1-14, 1994. 10, 11

[15] Costa, D. G. and Magalhães, C. A.: A Unified Approach to a Class of Strongly Indefinite Functionals. Journal of Differential Equations 125, 521-547, 1996. 2, 4

[16] Costa, D. G. and Magalhães, C. A.: A Variational Approach to Non-cooperative Elliptic Systems. Nonlinear Analysis, Theory, Methods \& Applications, 25 No 7, 699-715, 1995. 10, 11

[17] Costa, D. G. and Tehrani, H.: Existence and Multiplicity Results for a Class of Schrödinger Equations with Indefinite Nonlinearities. Adv. in Differential Equations 8, 1319-1340, 2003. 6, 44, 46

[18] Coti-Zelati Sissa, V. and Rabinowitz, P. H.: Homoclinic Type Solutions for a Semilinear Elliptic PDE on $\mathbb{R}^{N}$. Communications on Pure and Aplied Mathematics 45, 1217-1269, 1992.

[19] De Figueiredo, D. G. and Ding, Y.: Strongly Indefinite Functionals and Multiple Solutions of Elliptic Systems. Transactions of the American Mathematical Society 365 No. 7, 2973-2989, 2003. 11

[20] Ding, Y. and Jeanjean, L.: Homoclinic Orbits for a Non-periodic Hamiltonian System. Journal of Differential Equations 237, 473-490, 2007. 3

[21] Ding, Y. and Ruf, B.: Solutions of a Nonlinear Dirac Equation with External Fields. Arch. Rational Mech. Anal. 190, 57-82, 2008. 3 
[22] Edelson, A. L. and Stuart, C. A.: The Principle Branch of Solutions of a Nonlinear Elliptic Eigenvalue Problem on $\mathbb{R}^{N}$. Journal of Differential Equations 124, 279-301, 1996. 6. 7

[23] Egorov, Y. and Kondratiev, V.: On Spectral Theory of Elliptic Operators vol 89, Birkhäuser Verlag, 1996. 44, 102

[24] Fan, M., Wang, J., Xiao, L. and Zhang, F.: Existence and multiplicity of semiclassical solutions for asymptotically Hamiltonian elliptic systems. J. of Math. Anal. Appl. 339, 340-351, 2013. 11.

[25] Gladwell, G. M. L.: Inverse Problems in Scattering: An Introduction, Solid Mechanics and its Applications vol 23, Springer Science + Business Media Dordrecht, 1993.

[26] Hislop, P. D. and Sigal, I. M.: Introduction to Spectral Theory with Applications to Schrödinger Equations, Springer-Verlag, New York, Inc, 1996. 66

[27] Jeanjean, L.: On the Existence of Bounded Palais-Smale Sequences and Application to a Landesman-Lazer Type Problem Set on $\mathbb{R}^{N}$. Proc. Roy. Soc. Edinburgh 129A, 787-809, 1999. 3

[28] Jeanjean, L. and Tanaka, K.: A Positive solution to an Asymptotically Linear Elliptic Problem on $\mathbb{R}^{N}$ Autonomous at Infinity. ESAIM: Cont. Opt. Calc. Var. 7, 597-614, 2002. 5

[29] Jeanjean, L. and Tanaka, K.: Singularly Perturbed Elliptic Problems with Superlinear or Asymptotically Linear Nonlinearities. Calculus of Variations 21, 287-318, 2004. 11, 92

[30] Krasnoselski, M. A.: Topological Methods in the Theory of Nonlinear Integral Equations, New York, Macmillan, 1964.

[31] Kryszewski, W. and Szulkin, A.: Generalized Linking Theorem with an Application to Semilinear Schrödinger Equation. Adv. Differ. Equ. 3, 441-472, 1998. 2, 3,6

[32] Li, G. and Szulkin A.: An Asymptotically Periodic Schrödinger Equation with Indefinite Linear Part. Communications in Contemporary Mathematics 4 No. 4, 763-776, 2002. 2

[33] Li, G. and Wang, C.: The Existence of a Nontrivial Solution to a Nonlinear Elliptic Problem of Linking Type without the Ambrosetti-Rabinowitz Condition. Ann. Acad. Sci. Fenn. Math. 36, 461-480, 2011. 2, 5, 10 
[34] Lin, T. C. and Wei, J.: Symbiotic Bright Solitary Wave Solutions of Coupled Nonlinear Schrödinger equations. Nonlinearity 19, 2755-2773, 2006. 2, 5, 10

[35] Lions, P. L.: The Concentration-compactness Principle in the Calculus of Variations. The Locally Compact Case. Ann. Inst. Henri Poincaré, Analyse Non Linéaire 1, 109145 and 223-283, 1984. 52

[36] Maia, L. de A., Oliveira Junior, J. C. and Ruviaro, R.: A Non-periodic and Asymptotically Linear Indefinite Variational Problem in $\mathbb{R}^{N}$. Indiana University Mathematics Journal 66 No. 1, 31-54, 2017. 5, 7

[37] Oliveira, C. R.: Intermediate Spectral Theory and Quantum Dynamics, Progress in Mathematical Physics vol 54, Birkhäuser, 2009. 66, 102

[38] Palais, R. S.: The Principle of Symmetric Criticality. Comm. Math. Phys. 69, 19-30, 1979. 68, 78

[39] Pankov, A. A.: Lecture Notes on Schrödinger Equations, Nova Science Publishers, 2007. 46, 60

[40] Pankov, A. A.: Periodic Nonlinear Schrödinger Equation with Application to Photonic Crystals. Milan Journal of Mathematics 73, 259-287, 2005. 1

[41] Pomponio, A.:An asymptotically linear non-cooperative elliptic system with lack of compactness. The Royal Society, Mathematical, Physical and Engineering Sciences 459 No. 2037, 2265-2279, 2007. 11

[42] Rabelo, P.: Existence and Multiplicity of Solutions for a Class of Elliptic Systems in $\mathbb{R}^{N}$. Nonlinear Analysis 71, 2585-2599, 2009. 80, 82, 83, 91

[43] Rabinowitz, P. H.: Minimax Methods in Critical Point Theory with Applications to Differential Equations, American Mathematical Society, 1984. 27, 36, 103

[44] Reed, M. and Simon, B.: Methods of Modern Mathematical Physics, Analysis of Operators, Vol. IV, Academic Press, New York, 1978. 28, 37, 60, 66, 103

[45] Schechter, M.: Global Solutions of Nonlinear Schrödinger Equations. Calculus of Variations 56:40, 2017. 3

[46] Schechter, M.: Linking Methods in Critical Point Theory, Birkhäuser, Boston, 1999. 3 
[47] Schechter, M. and Zou, W.: An Infinite-dimensional Linking Theorem and Applications. Journal of Differential Equations 201, 324-350, 2004. 3

[48] Schechter, M and Zou, W.: Weak Linking Theorems and Schrödinger with Critical Sobolev Exponent. ESAIM Cont. Opt. Calc. Var. 9, 601-619, 2003. 3

[49] Silva, E. A. B.: Subharmonic Solutions for Sub-quadratic Hamiltonian Systems. Journal of Differential Equations 115 No.1, 120-145, 1995. 4

[50] Sirakov, B.: Existence and Multiplicity of Solutions of Semi-linear Elliptic Equations in $\mathbb{R}^{N}$. Calculus of Variations 11, 119-142, 2000. 8, 9, 11, 79, 80, 82, 91

[51] Strauss, W. A: Existence of Solitary Waves in Higher Dimensions. Comm. Math. Phys. 55, 149-162, 1977. 67, 69, 76, 103

[52] Stuart, C. A.: An Introduction to Elliptic Equations on $\mathbb{R}^{N}$, Trieste Notes, 1998. 44

[53] Stuart, C. A, and Zhou, H. S.: Applying the Mountain Pass Theorem to an Asymptotically Linear Elliptic Equation on $\mathbb{R}^{N}$. J. CommPDE 24, 1731-1758, 1999. 7, 8, 64

[54] Szulkin, A. and Weth, T.: Ground state solutions for some indefinite variational problems. J. Func. Anal. 257, 3802-3822, 2009. 1, 7

[55] Szulkin, A. and Zou, W.: Homoclinic Orbits for Asymptitotically linear Hamiltonian Systems. J. Func. Anal. 187, 25-41, 2001. 3

[56] Watanabe, T.: Radial Solutions with a Vortex to an Asymptotically Linear Elliptic Equation. Nonlinear Differential Equations and Applications 15, 387-411, 2018. 8

[57] Willem, M.: Minimax Theorems, vol 24, Birkhäuser, 1996. 2, 52, 103

[58] Wang, J., Xu, J. and Zhang, F.: Existence and Multiplicity of Solutions for Asymptotically Hamiltonian Elliptic Systems in $\mathbb{R}^{N}$. J. of Math. Anal. Appl. 367, 193-203, 2010 . 